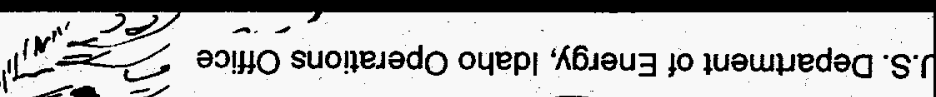

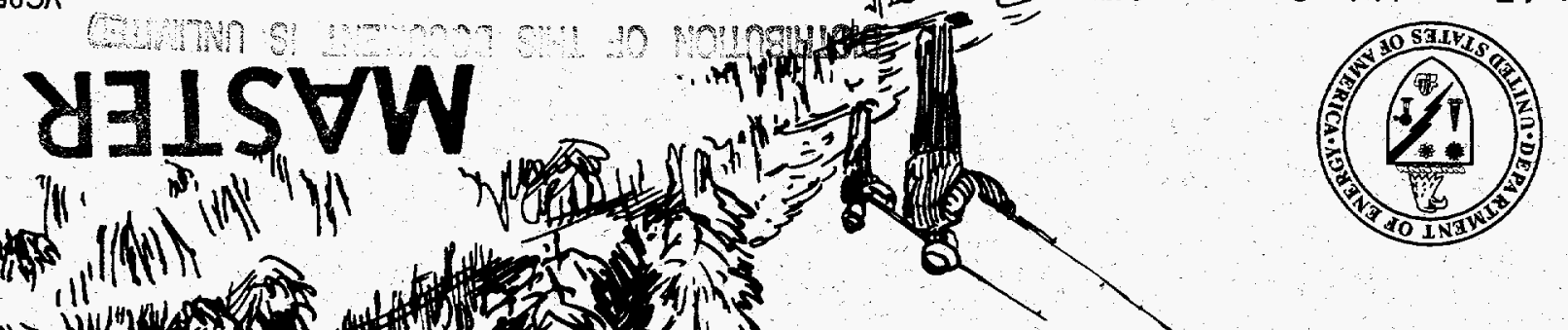

of

(y)

a)

(x) af wh

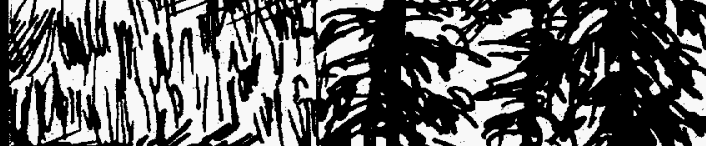

(D)

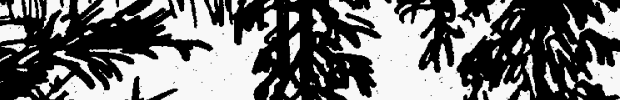

(n)

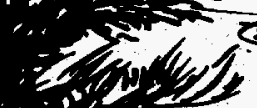

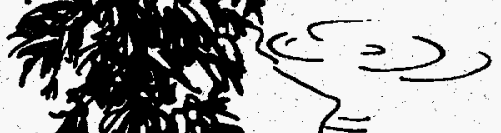

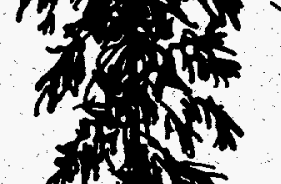

ming
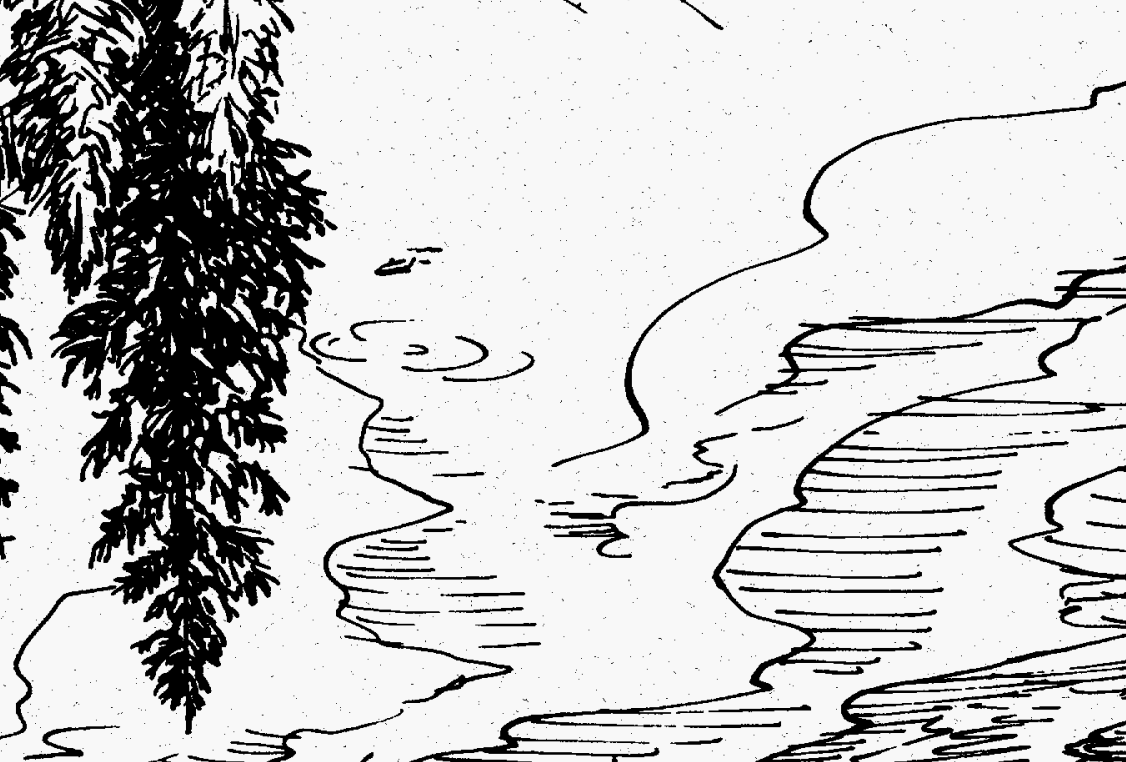

S 1.

(2.

Lin

$-20$

$=$

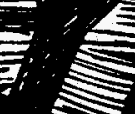

in.

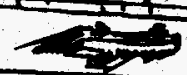

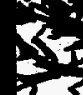

$\frac{E}{10}$

$=1$

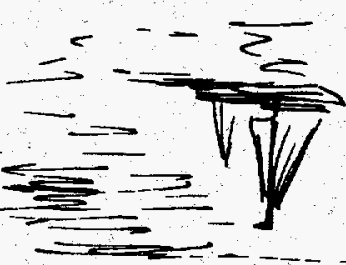

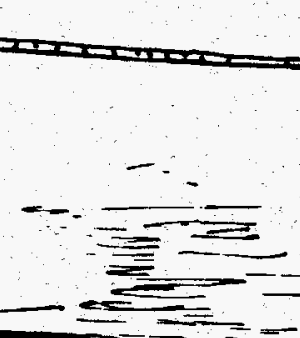

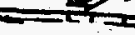

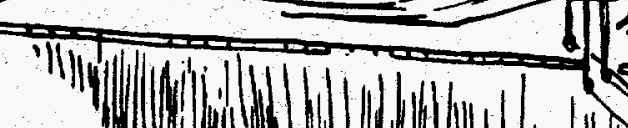

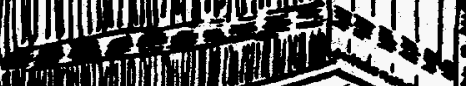

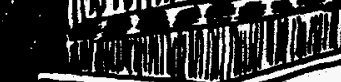
(1).

.

11.10

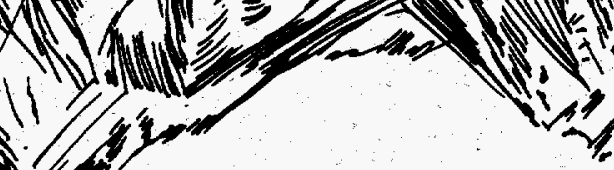

1150

S77assnyovsspH6

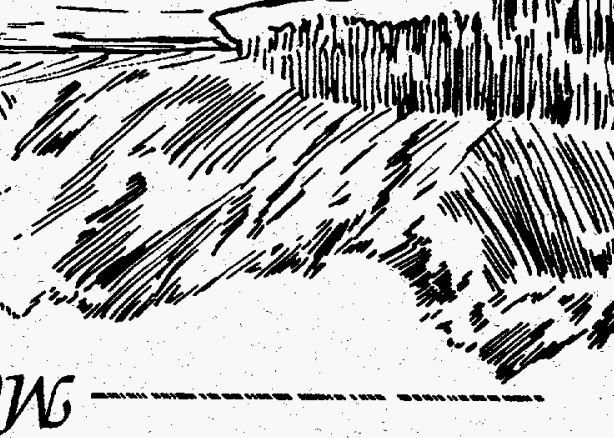

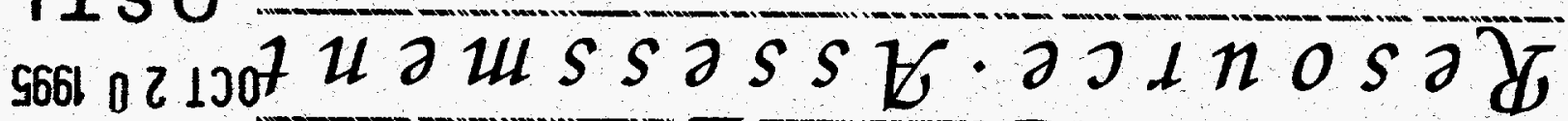

OजNISUS

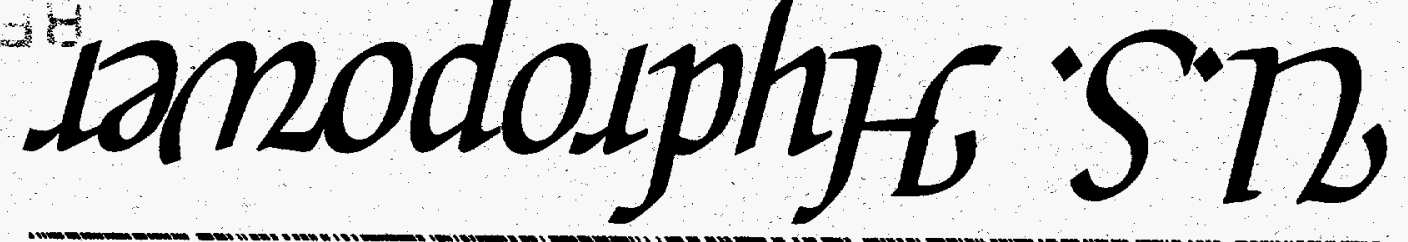




\section{DISCLAIMER}

Portions of this document may be illegible in electronic image products. Images are produced from the best available original document. 


\title{
U.S. Hydropower Resource Assessment for Massachusetts
}

\author{
Prepared by: \\ James E. Francfort \\ Project Manager: \\ Ben N. Rinehart \\ Published July 1995
}

\begin{abstract}
Idaho National Engineering Laboratory Renewable Energy Products Department Lockheed Idaho Technologies Company Idaho Falls, Idaho 83415
\end{abstract}





\begin{abstract}
The Department of Energy is developing an estimate of the undeveloped hydropower potential in the United States. The Hydropower Evaluation Software (HES) is a computer model that was developed by the Idaho National Engineering Laboratory for this purpose. The software measures the undeveloped hydropower resources available in the United States, using uniform criteria for measurement. The software was developed and tested using hydropower information and data provided by the Southwestern Power Administration. It is a menu-driven software program that allows the personal computer user to assign environmental attributes notential hydropower sites, calculate development suitability factors for each rased on the environmental attributes present, and generate reports based on : sitability factors. This report details the resource assessment results for the br. monwealth of Massachusetts.
\end{abstract}




\section{CONTENTS}

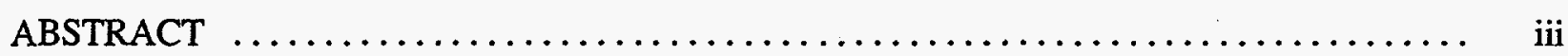

ACKNOWLEDGMENTS $\ldots \ldots \ldots \ldots \ldots \ldots \ldots \ldots \ldots \ldots \ldots \ldots \ldots \ldots \ldots \ldots \ldots \ldots \ldots \ldots \ldots$

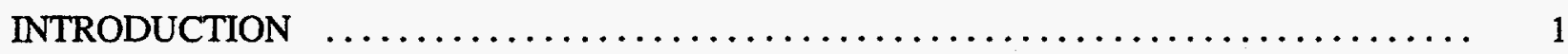

Model Development $\ldots \ldots \ldots \ldots \ldots \ldots \ldots \ldots \ldots \ldots \ldots \ldots \ldots \ldots \ldots \ldots \ldots \ldots \ldots$

Model Goal $\ldots \ldots \ldots \ldots \ldots \ldots \ldots \ldots \ldots \ldots \ldots \ldots \ldots \ldots \ldots \ldots \ldots \ldots \ldots \ldots \ldots \ldots$

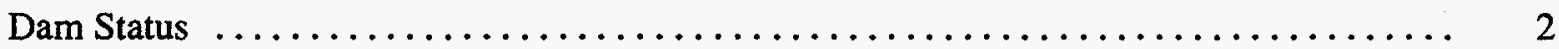

ASSESSMENT RESULTS $\ldots \ldots \ldots \ldots \ldots \ldots \ldots \ldots \ldots \ldots \ldots \ldots \ldots \ldots \ldots \ldots \ldots$

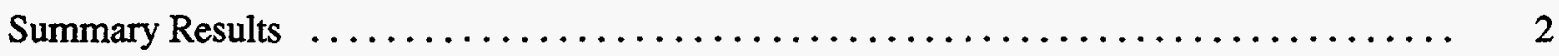

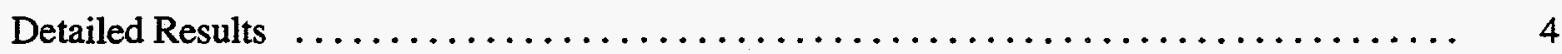

OBTAINING INDIVIDUAL STATE INFORMATION $\ldots \ldots \ldots \ldots \ldots \ldots \ldots \ldots \ldots \ldots \ldots$

ADDITIONAL HYDROPOWER EVALUATION SOFTWARE INFORMATION $\ldots \ldots \ldots \ldots$

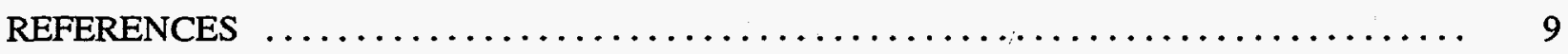

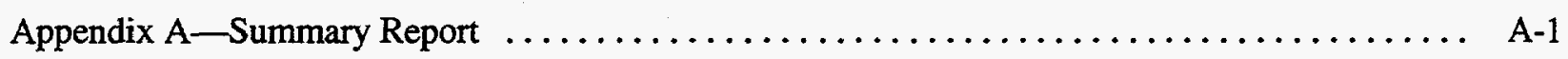

Appendix B-River Basins Report $\ldots \ldots \ldots \ldots \ldots \ldots \ldots \ldots \ldots \ldots \ldots \ldots \ldots \ldots \ldots \ldots \ldots \ldots \ldots \ldots$

Appendix $\mathrm{C}-$ Massachusetts Sites List $\ldots \ldots \ldots \ldots \ldots \ldots \ldots \ldots \ldots \ldots \ldots \ldots \ldots \ldots \ldots \ldots \ldots$

Appendix D-Individual Resource Database List $\ldots \ldots \ldots \ldots \ldots \ldots \ldots \ldots \ldots \ldots \ldots \ldots \ldots \ldots$

\section{FIGURES}

1. Number of sites with HES-modeled undeveloped hydropower potential $\ldots \ldots \ldots \ldots \ldots \ldots$

2. The HES-modeled undeveloped hydropower potential and the non-modeled

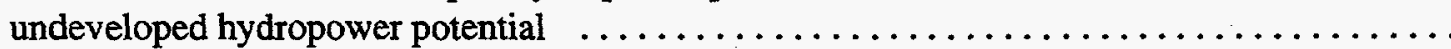

3. The number of sites with undeveloped hydropower potential and the total megawatts of HES-modeled undeveloped hydropower potential $\ldots \ldots \ldots \ldots \ldots \ldots \ldots \ldots \quad 4$

4. Lowell Hydroelectric Project, Lowell Massachusetts $\ldots \ldots \ldots \ldots \ldots \ldots \ldots \ldots \ldots \ldots$

5. Number of sites with undeveloped hydropower potential in each of the Massachusetts river basins

6. Megawatts of Hydropower Evaluation Software modeled undeveloped hydropower potential in the Massachusetts river basins 


\section{TABLES}

1. Summary of undeveloped hydropower potential for Massachusetts $\ldots \ldots \ldots \ldots \ldots \ldots \ldots$ 


\section{ACKNOWLEDGMENTS}

The author thanks Peggy A. M. Brookshier, John V. Flynn and S. J. Seymour of the Department of Energy and Raul Silva of the Commonwealth of Massachusetts for their active participation and timely comments.

\section{DISCLAIMER}

This report was prepared as an account of work sponsored by an agency of the United States Government. Neither the United States Government nor any agency thereof, nor any of their employees, makes any warranty, express or implied, or assumes any legal liability or responsibility for the accuracy, completeness, or usefulness of any information, apparatus, product, or process disclosed, or represents that its use would not infringe privately owned rights. Reference herein to any specific commercial product, process, or service by trade name, trademark, manufacturer, or otherwise does not necessarily constitute or imply its endorsement, recommendation, or favoring by the United States Government or any agency thereof. The views and opinions of authors expressed herein do not necessarily state or reflect those of the United States Government or any agency thereof. 


\section{U.S. Hydropower Resource Assessment for Massachusetts}

\section{INTRODUCTION}

In June 1989, the U.S. Department of Energy initiated the development of a National Energy Strategy to identify the energy resources available to support the expanding demand for energy in the United States. Public hearings conducted as part of the strategy development process indicated that undeveloped hydropower resources were not well defined. As a result, the Department of Energy established an interagency Hydropower Resource Assessment Team to ascertain the undeveloped hydropower potential. In connection with these efforts by the Department of Energy, the Idaho National Engineering Laboratory designed the Hydropower Evaluation Software (HES), which has been used to perform a resource assessment of the undeveloped conventional hydropower potential in Massachusetts (as well as several other states). This report presents the results of the hydropower resource assessment for the Commonwealth of Massachusetts. Undeveloped pumped storage hydropower potential is not included.

The HES was developed as a tool to measure undeveloped hydropower potential regionally or by state. The software is not intended to provide precise development factors for individual sites, but to provide regional or state totals. Because the software was developed as a generic measurement tool encompassing national issues, regional and state totals must be considered judiciously; various local issues may skew undeveloped hydropower potential totals. The information for the resource assessment was compiled from the Federal Energy Regulatory Commission's Hydroelectric Power Resources Assessment database and several other sources. Refer to DOE/ID-10338, the User's Manual (Francfort, Matthews, Rinehart 1991) for the specifics of the software and to DOE/ID-10430, the Status Report
(Francfort, Moore, Rinehart 1993) for an overview of all resource assessment activities to date.

\section{Model Development}

Hydropower Evaluation Software, both a probability-factor computer model and a database, is a menu-driven software program that is intended to be user-friendly. Computer screens and report generation capabilities were developed to meet the needs of users nationwide. The software uses environmental attribute data to generate an overall Project Environmental Suitability Factor (PESF) between 0.1 and 0.9 , where 0.9 indicates the highest likelihood of development and 0.1 indicates the lowest likelihood of development. The suitability factors depend on the unique environmental attributes of each potential site. They reflect the considerations that (a) environmental concerns can make a potential site unacceptable, prohibiting its development (for a suitability factor of 0.1 ), or (b) if there are no environmental concerns, there is no effect on the likelihood of site development (for a suitability factor of 0.9 ). A combination of attributes can result in a lower suitability factor because multiple environmental considerations would reduce the likelihood that a site may be developed to its physical potential.

\section{Model Goal}

The goal of the HES is to assemble an accurate resource database of all sites with undeveloped hydropower potential in the United States for use as a planning tool to determine the viable national hydropower potential. Undeveloped hydropower potential is not limited to the development of new sites; it also includes the development of additional hydropower generating capacity at sites that currently have hydropower but are not developed to their full potential. This undeveloped hydropower potential is a source of nonpolluting, renewable energy available to meet the growing 
power needs of the United States. The HES should help make this goal obtainable and ensure a set of uniform criteria for national assessment.

\section{Dam Status}

The effects of environmental attributes vary by dam status. The dam status classifications used are as follows:

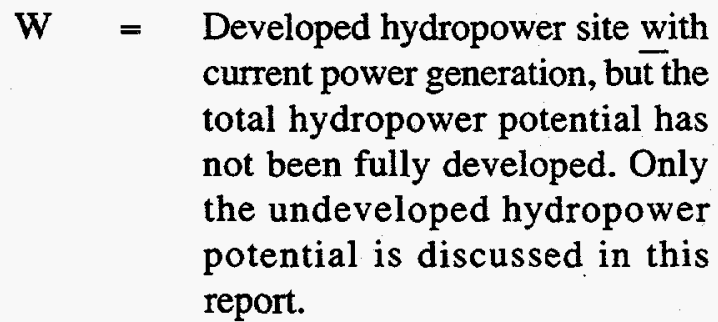

$W / O=$ Developed site without current power generation. The site has some type of developed impoundment or diversion structure, but no developed hydropower generating capability.

$\mathrm{U}=$ Undeveloped site. The site does not have power generation capability nor a developed impoundment or diversion structure.

\section{ASSESSMENT RESULTS}

\section{Summary Results}

A total of 130 sites (Table 1) have been identified and assessed for their undeveloped hydropower potential. The HES results for individual site capacities range from 15 kilowatts to 16.8 megawatts. Most of the sites have potential capacities of under 1 megawatt (Figure 1).

The non-modeled undeveloped hydropower potential for Massachusetts was identified as $\mathbf{3 2 5}$ megawatts. The HES results lower this estimate about $59 \%$ to 132 megawatts. The greatest reduction in undeveloped hydropower potential occurs at sites with no physical structures present. These undeveloped sites have an HES-modeled undeveloped hydropower potential of 56 megawatts, a $61 \%$ reduction in estimated undeveloped hydropower potential (Figure 2). The number of sites does not change, only the identified undeveloped hydropower potential is reassessed (Figure 3 ).

The 130 identified sites are located within 5 major river basins. An example of one of these sites is shown in Figure 4. The number of sites per major river basin range from 1 in the Hudson River Basin to 68 sites in the Connecticut River Basin (Figure 5). The Connecticut River Basin has the most undeveloped hydropower potential $(84 \mathrm{MW}$ ) of the Massachusetts river basins (Figure 6). The Connecticut River Basin total is driven by three sites that have more than half the basin's total undeveloped potential.

Table 1. Undeveloped hydropower potential summaries for Massachusetts The table contains the non-modeled undeveloped name plate potential, as well as the HES-modeled undeveloped hydropower potential totals.

\begin{tabular}{lccc}
\hline & Number of projects & $\begin{array}{c}\text { Name plate potential } \\
(\text { MW) }\end{array}$ & $\begin{array}{c}\text { HES modeled potential } \\
(\mathrm{MW})\end{array}$ \\
\cline { 2 - 4 } With Power & 12 & 28.2 & 14.5 \\
W/O Power & 87 & 117.9 & 62.1 \\
Undeveloped & 31 & 179.1 & 55.7 \\
State Total & 130 & 325.2 & 132.2 \\
\hline
\end{tabular}




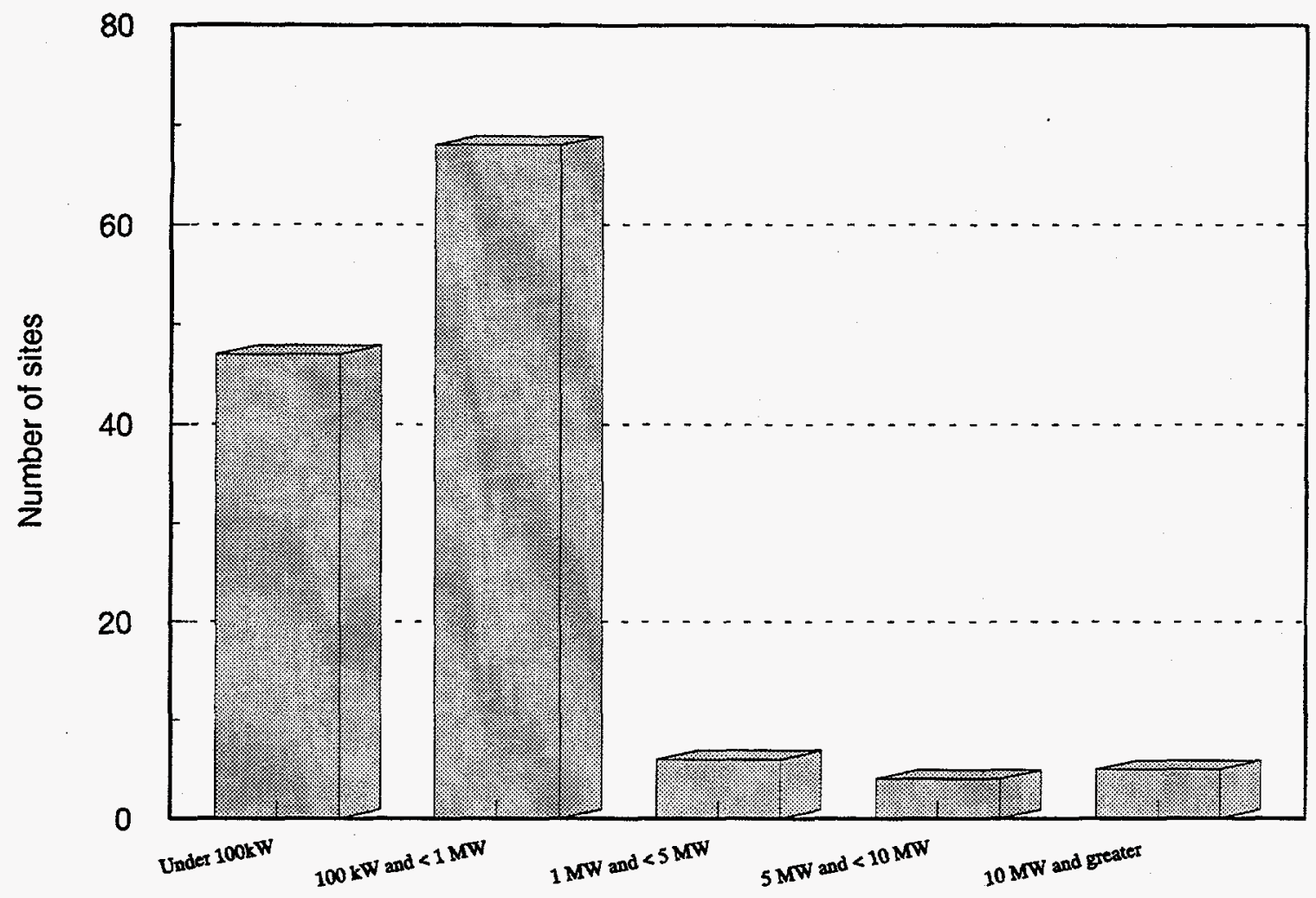

Figure 1. Number of sites with HES-modeled undeveloped hydropower potential.

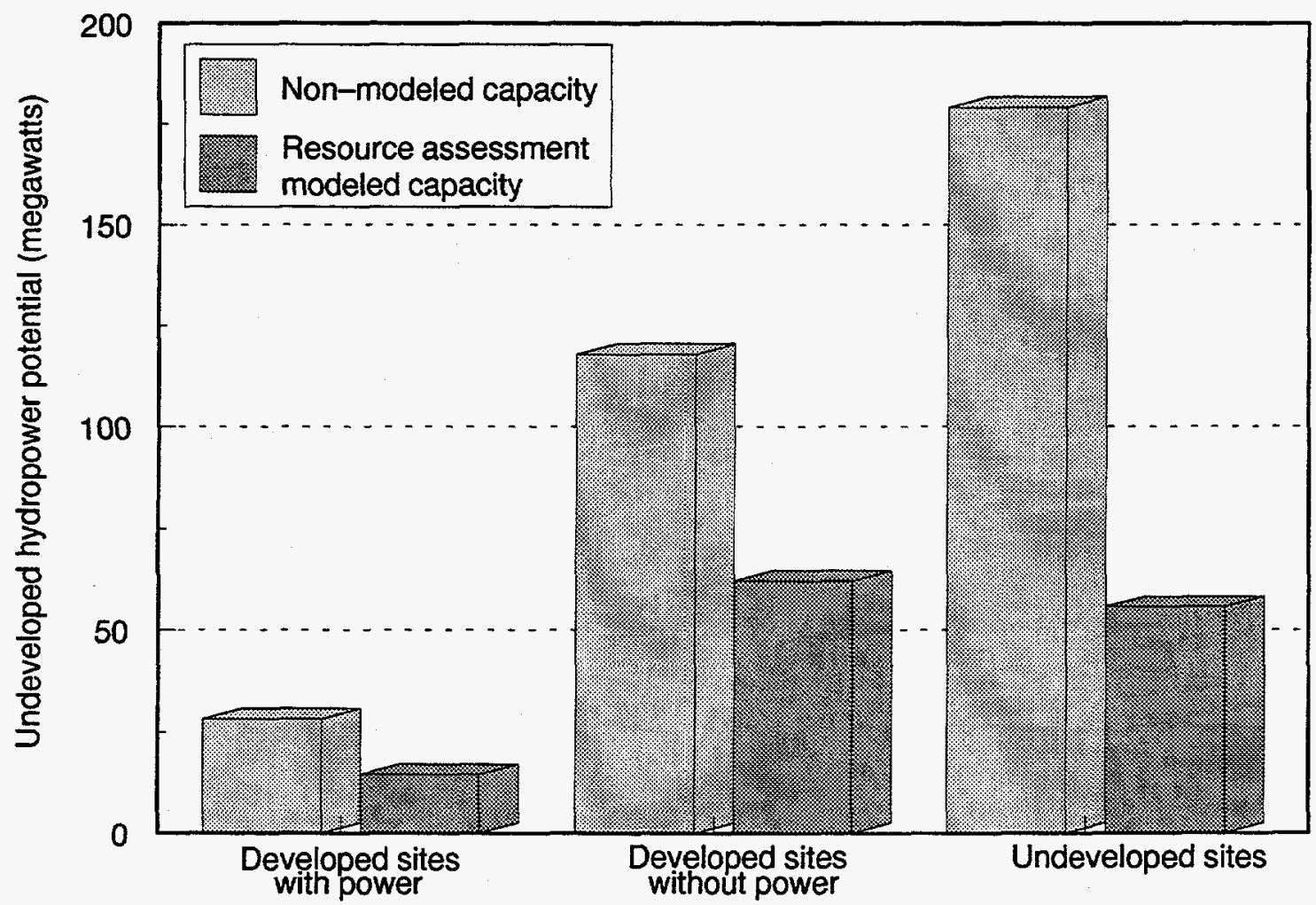

Figure 2. The HES-modeled undeveloped hydropower potential and the non-modeled undeveloped hydropower potential. 


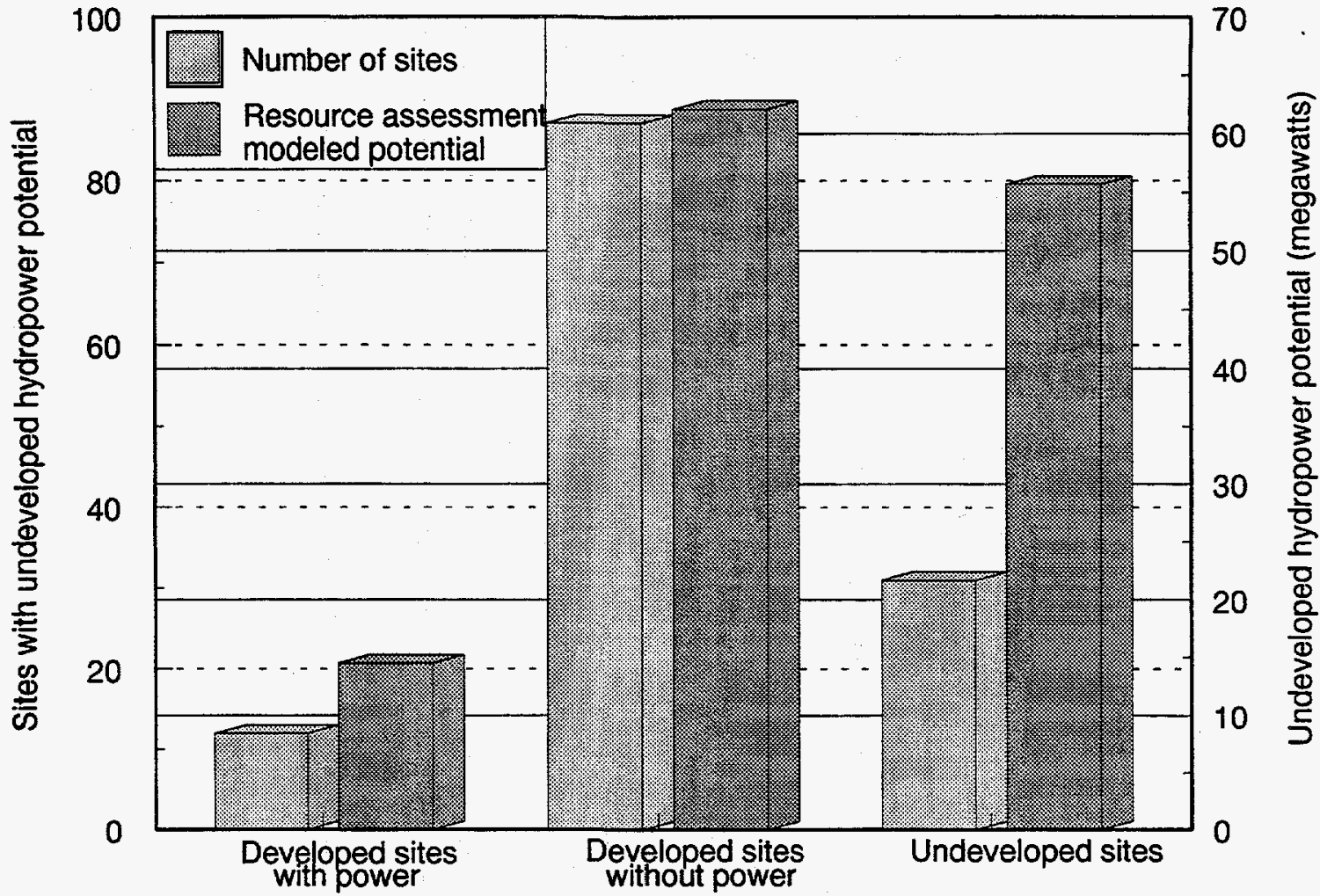

Figure 3. The number of sites with undeveloped hydropower potential and the total megawatts of HESmodeled undeveloped hydropower potential.

\section{Detailed Results}

The appendices contain, in the form of HESgenerated reports, detailed information of the undeveloped hydropower potential in Massachusetts. The appendices contain the following information:

Appendix A The undeveloped hydropower potential summary groups sites by dam status. The number of sites, non-modeled undeveloped hydropower potential, and HES. modeled undeveloped hydropower potential is provided based on the dam status.

Appendix B The hydropower resource assessment by river basin includes the project number, project name, stream name, dam status, nonmodeled undeveloped hydropower potential, and the HES- modeled undeveloped hydropower potential for each site. Subtotals are provided for each river basin.

Appendix C This is a list of the project numbers, plant name, stream name, if a site is Federally owned, nonmodeled undeveloped hydropower potential, and HESmodeled undeveloped hydropower potential. The sites are grouped by dam status.

Appendix D This section contains a resource database list for each of the 130 sites in Massachusetts. Information includes plant name, stream, state, county, river basin and owner names, project number, name plate and HES-modeled undeveloped hydropower potential, the unit and plant types, dam status, latitude, longitude, and 


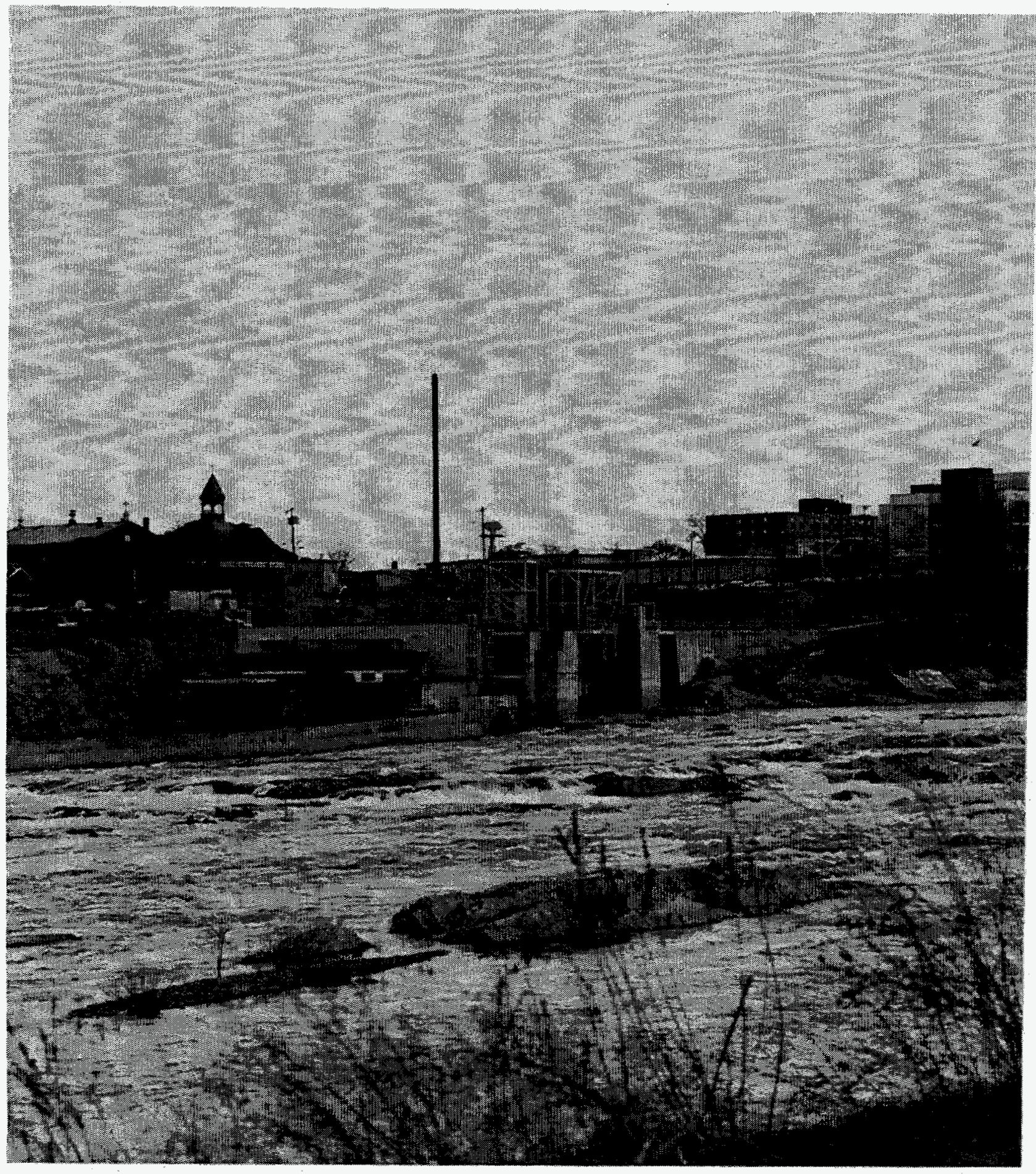

Figure 4. Lowell Hydroelectric Project, Lowell Massachusetts. 


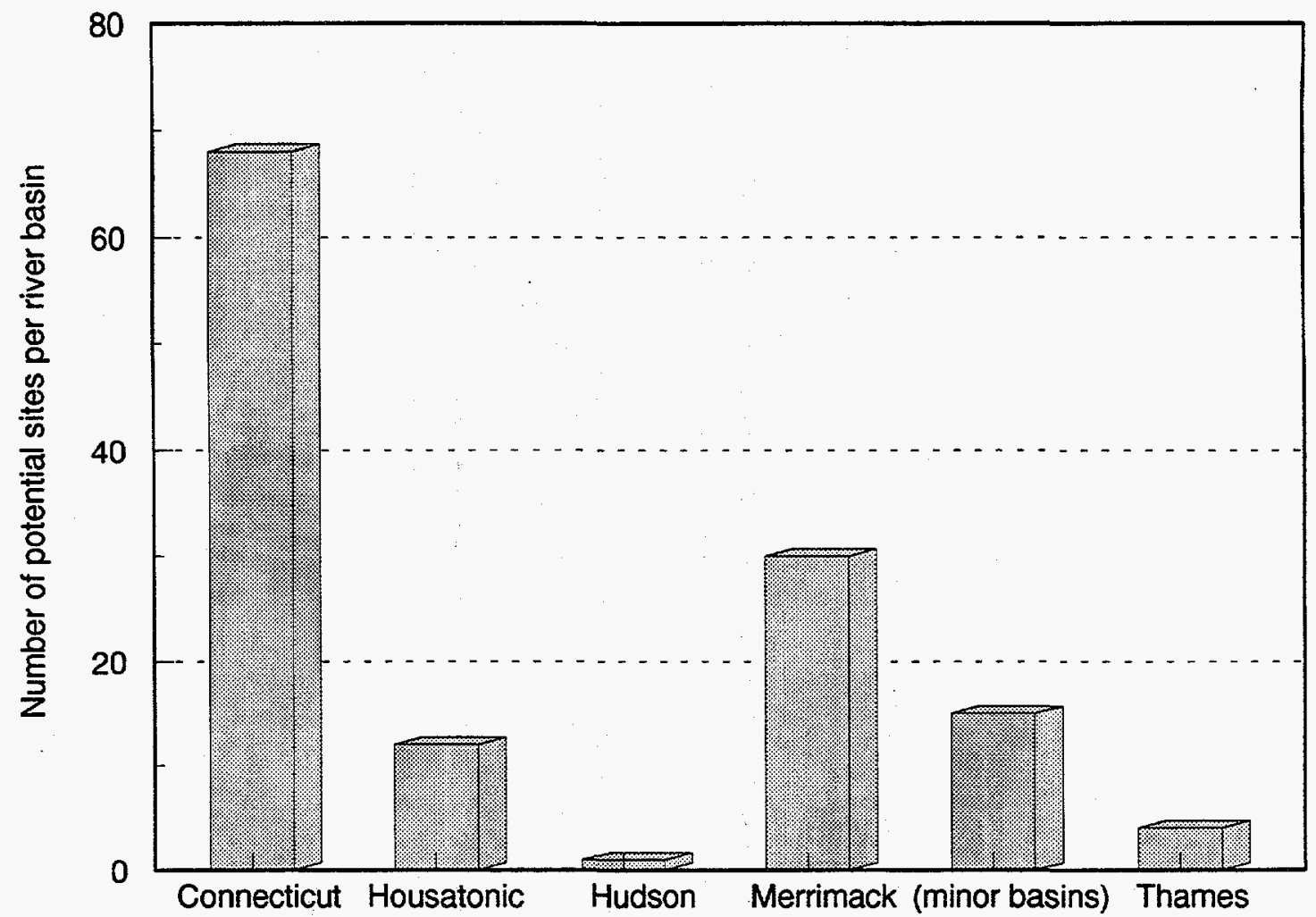

Figure 5. Number of sites with undeveloped hydropower potential in each of the Massachusetts river basins.

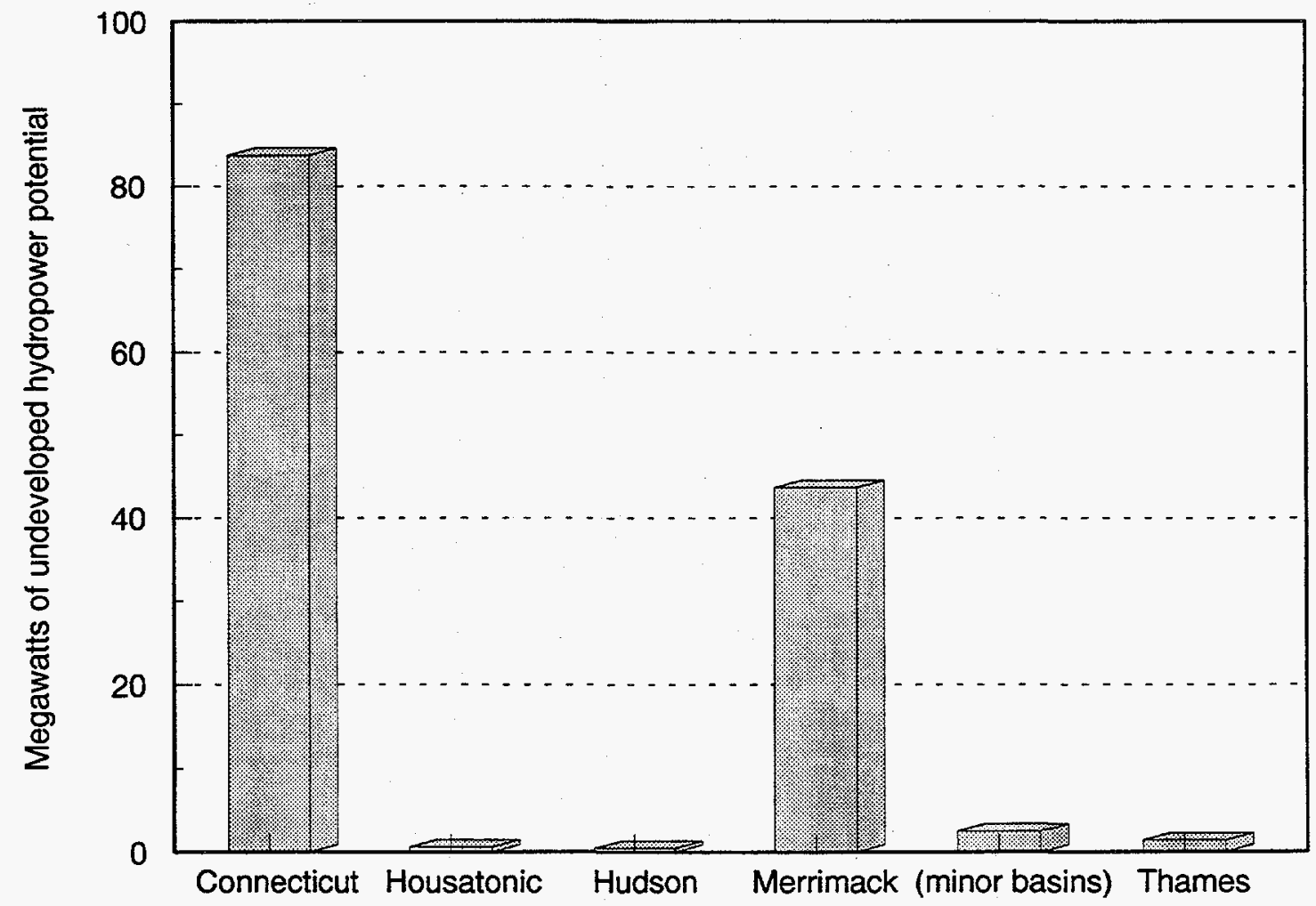

Figure 6. Megawatts of HES-modeled undeveloped hydropower potential in the Massachusetts river basins. 
the environmental factors that the HES uses to determine the project environmental suitability factor.

\section{OBTAINING INDIVIDUAL STATE INFORMATION}

Additional copies of the hydropower resource assessment results for individual states are available and can be obtained by writing or calling the National Technical Information Service (NTIS).

Telephone Orders-(703) 487-4650. NTIS sales desk and customer services are available between 8:30 a.m. and 5:00 p.m., Eastern Standard Time.

Fax-(703) 321-8547. Customers may fax their orders to NTIS. These orders may be charged to a NTIS deposit account, American Express, VISA, or MasterCard.

Mail Orders-Mail orders should be sent to National Technical Information Service, Document Sales, 5285 Port Royal Road, Springfield, VA 22161. Call the sales desk for prices before placing an order.

Method of Payment-Customers may pay for reports (and other NTIS products and services) by (a) credit card (American Express, Visa or MasterCard); (b) check or money order on a United States bank payable to NTIS; (c) a NTIS deposit account; or, (d) by asking to be billed (add $\$ 7.50$ per order), United States, Canada, and Mexico, only.

Handling Fee-A $\$ 3.00$ handling fee per total order applies to orders from the United States, Canada, and Mexico. Handling charges do not apply to rush order service or pick-up orders.

Postage and Shipping-Orders are shipped first class mail, or equivalent, to addresses in the United States, Canada, and Mexico.

Order Turnaround Time-Orders for technical reports generally are shipped within 2 to 8 days of receipt. For faster service, NTIS offers rush order service.
Rush Order Service-Call 1-800-533-NTIS. In Virginia, Canada, and Mexico call (703) 487-4700. For NTIS rush order service add $\$ 15.00$ per item. This guarantees that an order will be processed through NTIS within 24 hours of its receipt. These orders receive immediate, individual attention. The items ordered are delivered by first call mail. Call NTIS for information on rush order service for computer products.

For Help in Tracing an Order-Call (703) 487-4650 and request the customer service option.

\section{ADDITIONAL HYDROPOWER EVALUATION SOFTWARE INFORMATION}

Additional information concerning the HES can be obtained by contacting Ben Rinehart or Jim Francfort at the addresses provided below. Copies of the software and the User's Manual may also be obtained from these individuals.

Ben Rinehart

Idaho National Engineering Laboratory

P.O. Box 1625, M.S. 3830

Idaho Falls, ID 83415-3830

(208) 526-1002(208) 526-6787

Jim Francfort

Idaho National Engineering Laboratory

P.O. Box 1625, M.S. 3875

Idaho Falls, ID $83415-3875$

(208) 526-6787

Information concerning the Commonwealth of Massachusetts involvement with the resource assessment or about the identified sites may be obtained by contacting:

Raul Silva

Office of Dam Safety

Department of Environmental Management

Division of Resource Conservation

100 Cambridge St.

Boston, Massachusetts 02202

(617) 727-3160 


\section{REFERENCES}

Francfort, J. E., S. D. Matthews, and B. N. Rinehart, 1991, Hydropower Evaluation Software User's Manual, DOE/ID-10338, Idaho National Engineering Laboratory, Idaho Falls, Idaho.

Francfort, J. E., K. M. Moore, and B. N. Rinehart, 1993, Uniform Criteria for U.S. Hydropower Resource Assessment, Hydropower Evaluation Software Status Report, DOE/ID-10430, Idaho National Engineering Laboratory, Idaho Falls, Idaho. 
Appendix A

Summary Report

A-1 


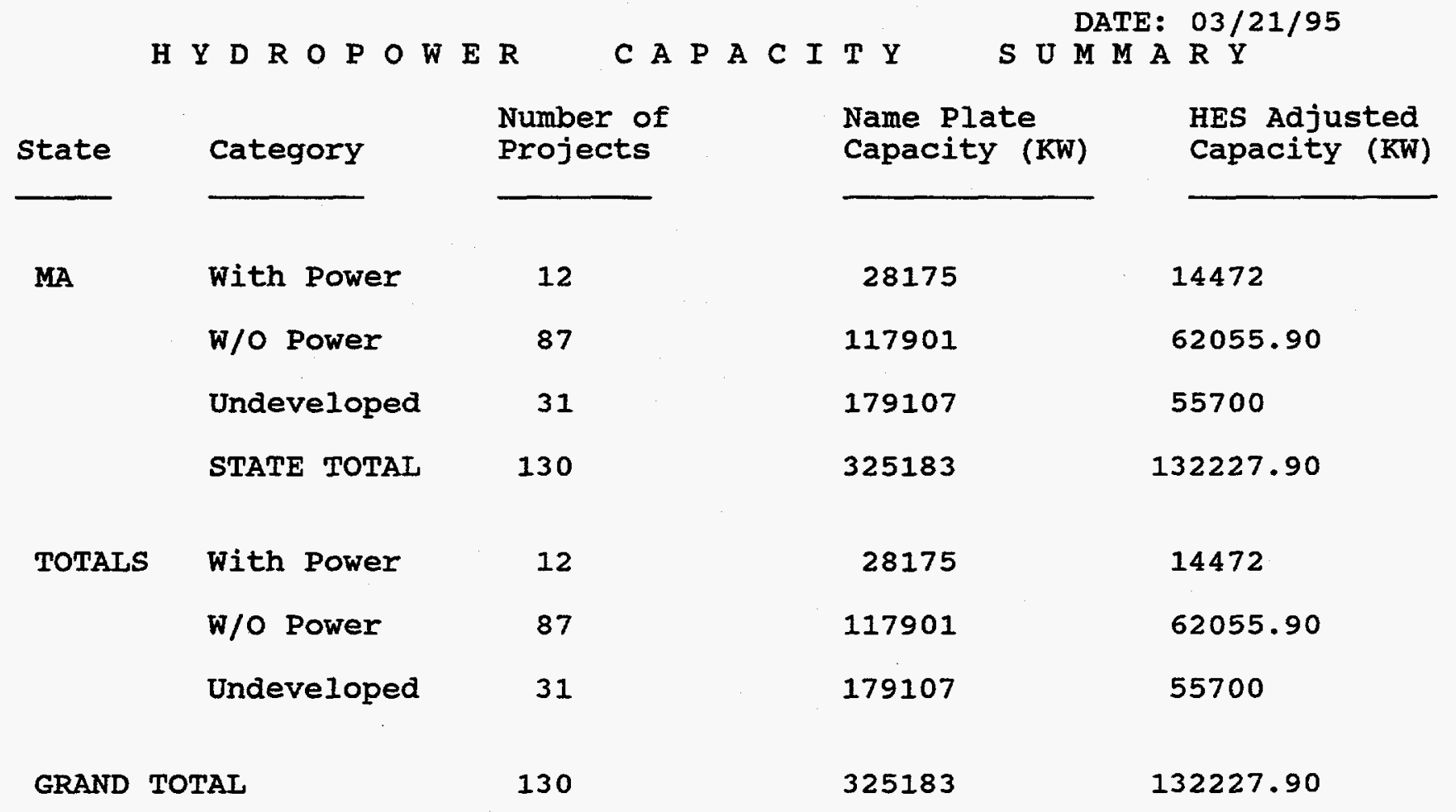




\section{Appendix B}

River Basins Report

B-1 


\begin{tabular}{|c|c|c|c|c|c|}
\hline & HYDROPOWER RESOURCE & ASSESSM & ENT BY RIVER & BASIN & \\
\hline $\begin{array}{l}\text { FERC } \\
\text { Number }\end{array}$ & $\begin{array}{c}\text { Plant Name/ } \\
\text { Stream }\end{array}$ & $\begin{array}{l}\text { Dam } \\
\text { stat }\end{array}$ & $\begin{array}{l}\text { Name Plate } \\
\text { Rating (KW) }\end{array}$ & PESF & PESF * KW \\
\hline $\begin{array}{l}* * \text { Rive } \\
05057\end{array}$ & $\begin{array}{l}\text { BaSin CONNECTICUT RIVER BASI } \\
\text { FACTORY HOLLOW } \\
\text { MILL R, CONNECTICUT R }\end{array}$ & N & 52.00 & 0.50 & 26.00 \\
\hline 09129 & $\begin{array}{l}\text { OTIS } \\
\text { FALI R,W BR FARMINGTON R }\end{array}$ & $\mathbf{U}$ & 500.00 & 0.10 & 50.00 \\
\hline MA016 & $\begin{array}{l}\text { WEST SPRINGFIELD } \\
\text { WESTFIELD } R\end{array}$ & U & 230.00 & 0.50 & 115.00 \\
\hline MA017 & $\begin{array}{l}\text { GRANVILLE } \\
\text { MUNN BK, LITTLE } R\end{array}$ & $\mathrm{U}$ & 120.00 & 0.90 & 108.00 \\
\hline MA018 & $\begin{array}{l}\text { THE GORGE } \\
\text { LITTLE R. }\end{array}$ & $\mathrm{U}$ & 490.00 & 0.90 & 441.00 \\
\hline MAO22 & $\begin{array}{l}\text { HUNTINGTON } \\
\text { WESTFIELD R }\end{array}$ & $\mathbf{U}$ & .6000 .00 & 0.50 & 3000.00 \\
\hline MA025 & $\begin{array}{l}\text { LUDLOW (RED BRIDGE) } \\
\text { CHICOPEE R }\end{array}$ & U & 1185.00 & 0.50 & 592.50 \\
\hline 09720 & $\begin{array}{l}\text { TEXTILE LOWER } \\
\text { SWIFT R }\end{array}$ & $\mathbf{U}$ & 150.00 & 0.90 & 135.00 \\
\hline 09640 & $\begin{array}{l}\text { TEXTILE UPPER } \\
\text { SWIFT R }\end{array}$ & $\mathrm{U}$ & 150.00 & 0.90 & 135.00 \\
\hline 05873 & $\begin{array}{l}\text { BARRE FALLS } \\
\text { WARE } R, C H I C O P E E ~ R\end{array}$ & $\mathbf{U}$ & 550.00 & 0.50 & 275.00 \\
\hline MA027 & $\begin{array}{l}\text { TIGHE CARMODY } \\
\text { MANHAN } R\end{array}$ & U & 230.00 & 0.50 & 115.00 \\
\hline 03123 & $\begin{array}{l}\text { STILLWATER BRIDGE } \\
\text { DEERFIELD } R\end{array}$ & U & 7000.00 & 0.25 & 1750.00 \\
\hline MA031 & $\begin{array}{l}\text { GARDNER FALLS } \\
\text { DEERFIELD } R\end{array}$ & U & 1720.00 & 0.25 & 430.00 \\
\hline MA033 & $\begin{array}{l}\text { CHARLEMONT } \\
\text { DEERFIELD R }\end{array}$ & $U$ & 20000.00 & 0.25 & 5000.00 \\
\hline MA035 & $\begin{array}{l}\text { ZOAR } \\
\text { DEERFIELD } R\end{array}$ & $\mathrm{U}$ & 20000.00 & 0.25 & 5000.00 \\
\hline MA037 & $\begin{array}{l}\text { TURNERS FALLS } \\
\text { CONNECTICUT R }\end{array}$ & $\mathrm{U}$ & 66000.00 & 0.25 & 16500.00 \\
\hline
\end{tabular}




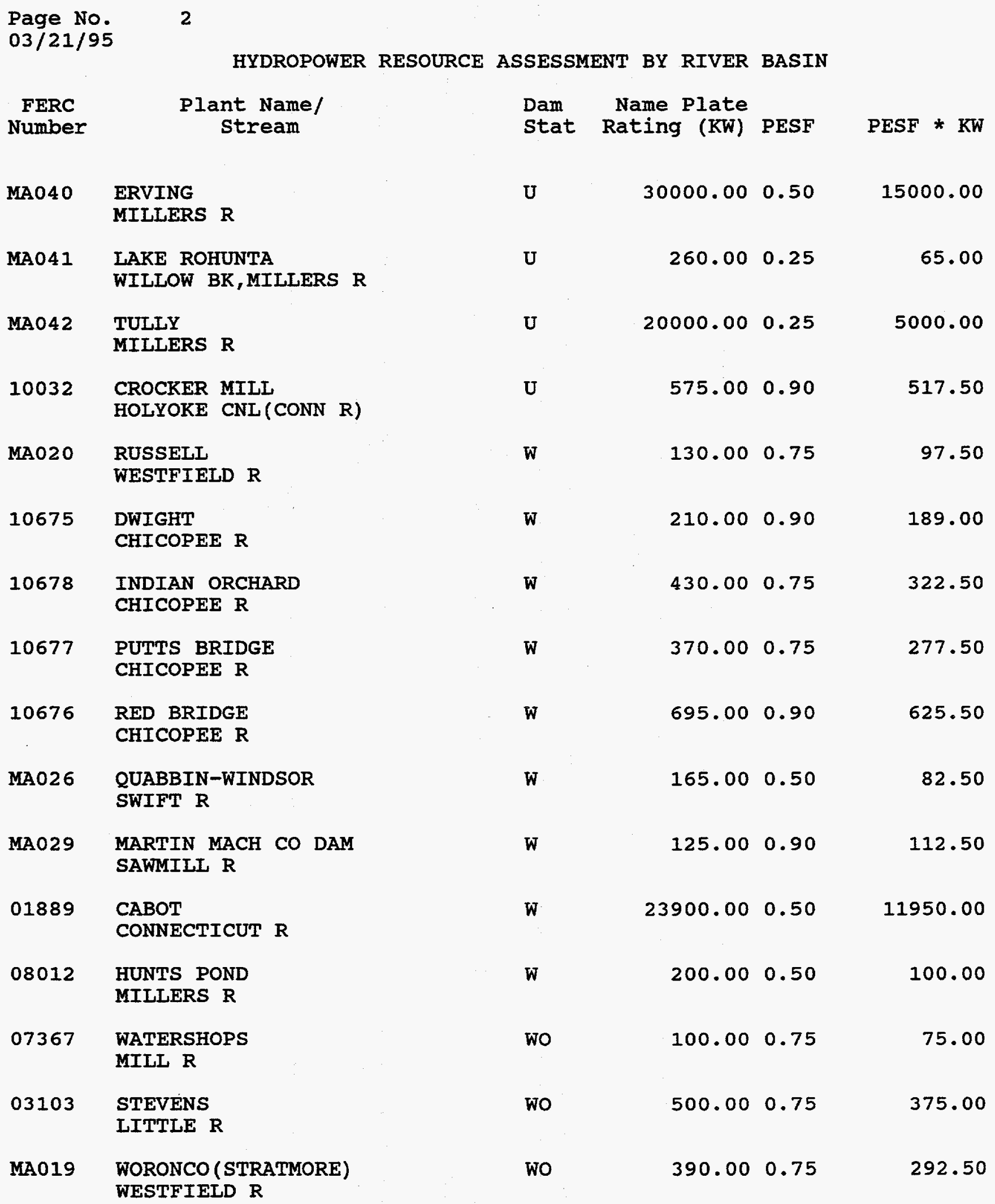




\begin{tabular}{|c|c|c|c|c|c|}
\hline & HYDROPOWER RESOURCE & ASSESSM & ENT BY RIVER & BASIN & \\
\hline $\begin{array}{l}\text { FERC } \\
\text { Number }\end{array}$ & $\begin{array}{l}\text { Plant Name/ } \\
\text { Stream }\end{array}$ & $\begin{array}{l}\text { Dam } \\
\text { stat }\end{array}$ & $\begin{array}{r}\text { Name Plate } \\
\text { Rating }(\mathrm{KW})\end{array}$ & PESF & PESF * KW \\
\hline MA021 & $\begin{array}{l}\text { RUSSELL (CRESCENT MILLS) } \\
\text { WESTFIELD R }\end{array}$ & พ० & 1125.00 & 0.75 & 843.75 \\
\hline 08350 & $\begin{array}{l}\text { LITTLEVILLE } \\
\text { M BR WESTFIELD R }\end{array}$ & พั & 1060.00 & 0.50 & 530.00 \\
\hline 09895 & $\begin{array}{l}\text { KNIGHTVILLE } \\
\text { WESTFIELD R }\end{array}$ & พอ & 3000.00 & 0.50 & 1500.00 \\
\hline MA023 & $\begin{array}{l}\text { INDIAN ORCHARD } \\
\text { CHICOPEE R }\end{array}$ & wo & 785.00 & 0.75 & 588.75 \\
\hline MAO24 & $\begin{array}{l}\text { PUTTS BRIDGE } \\
\text { CHICOPEE R }\end{array}$ & wo & 2345.00 & 0.75 & 1758.75 \\
\hline 09615 & $\begin{array}{l}\text { THREE RIVERS } \\
\text { CHICOPEE R }\end{array}$ & พ० & 2360.00 & 0.50 & 1180.00 \\
\hline 09432 & $\begin{array}{l}\text { MANHAN } \\
\text { MANHAN R }\end{array}$ & พั & 142.00 & 0.90 & 127.80 \\
\hline 09332 & $\begin{array}{l}\text { PARADISE } \\
\text { MILL R }\end{array}$ & พ० & 75.00 & 0.50 & 37.50 \\
\hline 09330 & $\begin{array}{l}\text { NONOTUCKET STREET } \\
\text { MILL R }\end{array}$ & พO & 75.00 & 0.75 & 56.25 \\
\hline 09276 & $\begin{array}{l}\text { COOKS } \\
\text { MILL R }\end{array}$ & พо & 180.00 & 0.50 & 90.00 \\
\hline 09331 & $\begin{array}{l}\text { SPRING STREET } \\
\text { MILL R }\end{array}$ & พอ & 100.00 & 0.90 & 90.00 \\
\hline 09329 & $\begin{array}{l}\text { LEEDS } \\
\text { MILL R }\end{array}$ & WO & 75.00 & 0.75 & 56.25 \\
\hline 06966 & $\begin{array}{l}\text { BRASSWORKS } \\
\text { MIIL R }\end{array}$ & พо & 75.00 & 0.75 & 56.25 \\
\hline MAO28 & $\begin{array}{l}\text { ROARING BROOK DAM } \\
\text { ROARING BR, MILI R }\end{array}$ & Wo & 52.00 & 0.90 & 46.80 \\
\hline 05462 & $\begin{array}{l}\text { LAKE WARNER } \\
\text { MILI R, CONNECTICUT } R\end{array}$ & พั & 30.00 & 0.75 & 22.50 \\
\hline MA030 & $\begin{array}{l}\text { DENISON DAM } \\
\text { GREEN R, DEERFIELD R }\end{array}$ & พ० & 115.00 & 0.50 & 57.50 \\
\hline
\end{tabular}


Page No.

$03 / 21 / 95$

FERC

Number

05666B

GREEN ONE (MILL 1)

GREEN R, DEERFIELD $R$

05666A GREEN TWO(MILL 2)

GREEN R, DEERFIELD $R$

09066 CONWAY

SOUTH $R, D E E R F I E L D ~ R$

MA032 DEERFIELD 3

MA034 MILL BROOK

MILL BK, DEERFIELD R

MA036 SHERMAN

DEERFIELD $R$

08449 BERNARDSTON GRAIN MILL

FALL $R$

MA038 BOOK BINDERY POND

MILLERS $R$

MA039 MILLERS FALLS ONE

MILLERS $R$

08234A MILLERS ONE

MILLERS $\mathbf{R}$

08234B MILLERS TWO

MILLERS $R$

08234C MILLERS THREE

MILLERS $\mathbf{R}$

08234D MIILERS FOUR

MILLERS $R$

08234E MILLERS FIVE

MILLERS $R$

06096 NEW HOME SOUTH

MILLERS $R$

03423 BIRCH HILL MIILERS $R$
Dam Name Plate

stat Rating (KW) PESF PESF * KW

พั

$71.00 \quad 0.50$

35.50

พั

$106.00 \quad 0.50$

53.00

พo

$350.00 \quad 0.50$

175.00

wo

$1135.00 \quad 0.50$

567.50

wo

$100.00 \quad 0.50$

50.00

พo

$3020.00 \quad 0.50$

1510.00

wo

$80.00 \quad 0.90$

72.00

wo

$1000.00 \quad 0.75$

750.00

wo

$500.00 \quad 0.75$

375.00

wo

$1090.00 \quad 0.50$

545.00

wo

$1950.00 \quad 0.50$

975.00

พo

$1090.00 \quad 0.50$

545.00

wo

$800.00 \quad 0.50$

400.00

wo

$800.00 \quad 0.50$

400.00

wo

$250.00 \quad 0.75$

187.50

wo

$670.00 \quad 0.75$

502.50 


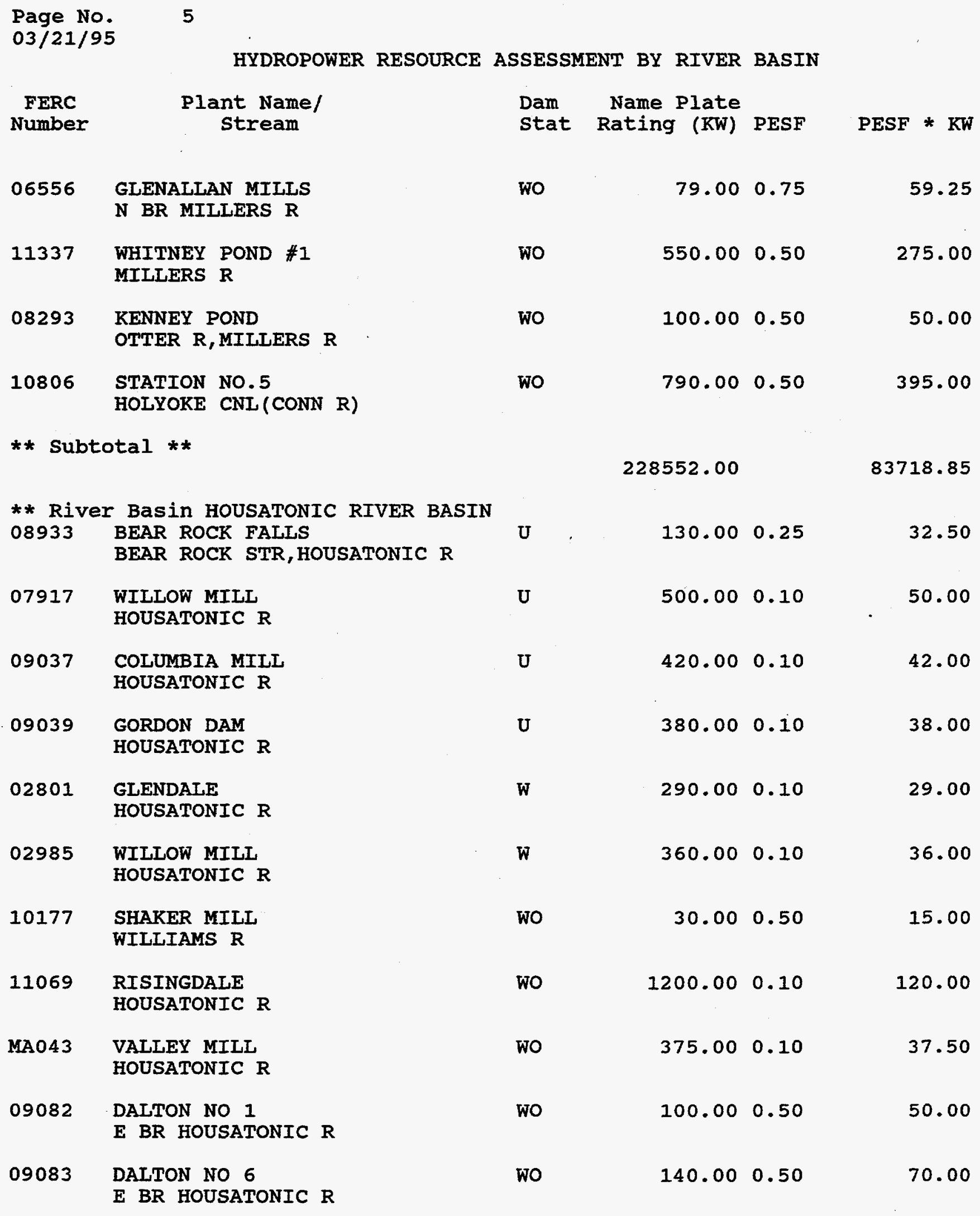


Page No.

$03 / 21 / 95$

FERC

Number

09081

CENTER POND

E BR HOUSATONIC R

** Subtotal **

** River Basin HUDSON RIVER BASIN

11306 ECLIPSE DAM

NBR HOOSIC RIVER

** Subtotal **

** River Basin MERRIMACK RIVER BASIN

09616

DELANEY

SQUANNACOOK $R$

MA007 LANCASTER MILL POND

NASHUA $R$

10688 COSGROVE

COSGROVE AQUE(WACHUSETT RES)

10580 POW WOW RIVER

POWWOW R,MERRIMACK $R$

09978 LAKE GARDNER

POWWOW $R$, MERRIMACK $R$

08641 BALLARDVALE

SHAWSHEEN $R$

08653 ANDOVER

SHAWSHEEN $R$

10563 GEO MAC MEMORIAI

WAMESIT CNL

11102 MIDDLESEX

CONCORD $\mathrm{R}$

08424 BILLERICA

CONCORD $R$

09288 SAXONVILLE

SUDBURY R

09287 STEARNS

SUDBURY $R$
Dam Name Plate

Stat Rating (KW) PESF PESF * KW

wo

$130.00 \quad 0.75$

97.50

4055.00

617.50

พั

$600.00 \quad 0.75$

450.00

600.00

450.00

U

$105.00 \quad 0.90$

94.50

U

$360.00 \quad 0.25$

90.00

W

$1300.00 \quad 0.50$

650.00

พั

$300.00 \quad 0.75$

225.00

wo

$120.00 \quad 0.75$

90.00

พo

$40.00 \quad 0.90$

36.00

wo

$50.00 \quad 0.90$

45.00

พอ

$376.00 \quad 0.90$

338.40

พо

$1300.00 \quad 0.50$

650.00

wo

$200.00 \quad 0.50$

100.00

wo

$150.00 \quad 0.50$

75.00

พั

$60.00 \quad 0.50$

30.00 


\begin{tabular}{|c|c|c|c|c|c|}
\hline & HYDROPOWER RESOURCE & ASSESSN & ENT BY RIVER & BASIN & \\
\hline $\begin{array}{l}\text { FERC } \\
\text { Number }\end{array}$ & $\begin{array}{c}\text { Plant Name/ } \\
\text { Stream }\end{array}$ & $\begin{array}{l}\text { Dam } \\
\text { Stat }\end{array}$ & $\begin{array}{l}\text { Name Plate } \\
\text { Rating (KW) }\end{array}$ & PESF & PESF * KW \\
\hline MA001 & $\begin{array}{l}\text { SUDBURY } \\
\text { STONY BK, SUDBURY R }\end{array}$ & พо & 265.00 & 0.50 & 132.50 \\
\hline 10012 & $\begin{array}{l}\text { WASHINGTON STREET } \\
\text { ASSABET } R\end{array}$ & wo & 100.00 & 0.50 & 50.00 \\
\hline MAO02 & $\begin{array}{l}\text { OLD GUARD LOCKS } \\
\text { MERRIMACK CNL (MERR R) }\end{array}$ & พo & 24600.00 & 0.50 & 12300.00 \\
\hline $\mathrm{MAO03}$ & $\begin{array}{l}\text { SWAMP LOCKS } \\
\text { MERRIMACK CNL (MERR R) }\end{array}$ & พั & 17900.00 & 0.50 & 8950.00 \\
\hline MA004 & $\begin{array}{l}\text { PAWTUCKET ( } 4 \text { ) } \\
\text { FOUR CANALS (MERR R) }\end{array}$ & wo & 906.00 & 0.50 & 453.00 \\
\hline MAO0 5 & $\begin{array}{l}\text { NORTH CANAI LOCKS } \\
\text { MERRIMACK CNL (MERR R) }\end{array}$ & พо & 33600.00 & 0.50 & 16800.00 \\
\hline 08652 & $\begin{array}{l}\text { PLEASANT STREET } \\
\text { BEAVER BK, MERRIMACK R }\end{array}$ & wo & 65.00 & 0.75 & 48.75 \\
\hline MA006 & $\begin{array}{l}\text { PEPPERELL } \\
\text { NASHUA } \mathbf{R}\end{array}$ & wo & 470.00 & 0.50 & 235.00 \\
\hline 04809 & $\begin{array}{l}\text { TOWNSEND HARBOR } \\
\text { SQUANNACOOK } R\end{array}$ & wo & 250.00 & 0.50 & 125.00 \\
\hline 10338 & $\begin{array}{l}\text { WEST GROTON } \\
\text { SQUANNACOOK R }\end{array}$ & wo & 210.00 & 0.90 & 189.00 \\
\hline 11001 & $\begin{array}{l}\text { NASHUA } \\
\text { NASHUA R }\end{array}$ & wo & 500.00 & 0.75 & 375.00 \\
\hline MA008 & $\begin{array}{l}\text { WACHUSETT } \\
S \text { BR NASHUA } R\end{array}$ & wo & 1070.00 & 0.50 & 535.00 \\
\hline MAOO9 & $\begin{array}{l}\text { MACTAGGARTS POND } \\
\text { PHILLIPS BK, N NASHUA R }\end{array}$ & พo & 250.00 & 0.75 & 187.50 \\
\hline 11111 & $\begin{array}{l}\text { CROCKER BURBANK MILI } 6 \\
\text { NASHUA } R\end{array}$ & wo & 100.00 & 0.75 & 75.00 \\
\hline 11166 & $\begin{array}{l}\text { SNOWS MILL POND } \\
\text { WHITMAN R, NASHUA } R\end{array}$ & wo & 350.00 & 0.90 & 315.00 \\
\hline 11058 & $\begin{array}{l}\text { FITCHBURG } 4 \\
\text { N NASHUA R }\end{array}$ & พо & 50.00 & 0.75 & 37.50 \\
\hline
\end{tabular}




\begin{tabular}{|c|c|c|c|c|c|}
\hline & HYDROPOWER RESOURCE & ASSESSN & ENT BY RIVER & BASIN & \\
\hline $\begin{array}{l}\text { FERC } \\
\text { Number }\end{array}$ & $\begin{array}{c}\text { Plant Name/ } \\
\text { stream }\end{array}$ & $\begin{array}{l}\text { Dam } \\
\text { stat }\end{array}$ & $\begin{array}{l}\text { Name Plate } \\
\text { Rating (KW) }\end{array}$ & PESF & $\mathrm{PESF} * \mathbf{K W}$ \\
\hline 11002 & $\begin{array}{l}\text { ARDEN MILL } \\
\text { NASHUA } R\end{array}$ & พ० & 200.00 & 0.75 & 150.00 \\
\hline MA010 & $\begin{array}{l}\text { FITCHBURG } \\
\text { N NASHUA R }\end{array}$ & wo & 250.00 & 0.75 & 187.50 \\
\hline ** Subt & stal ** & & 85497.00 & & 43569.65 \\
\hline $\begin{array}{l}\text { ** Rive } \\
\text { MA011 }\end{array}$ & $\begin{array}{l}\text { Basin MINOR RIVER BASINS } \\
\text { WATERTOWN } \\
\text { CHARIES R }\end{array}$ & $\mathrm{U}$ & 415.00 & 0.25 & 103.75 \\
\hline MA012 & $\begin{array}{l}\text { SOUTH WATUPPA POND } \\
\text { QUEQUECHAN. R }\end{array}$ & $\mathrm{U}$ & 270.00 & 0.90 & 243.00 \\
\hline 09606 & $\begin{array}{l}\text { QUEQUECHAN RIVER } \\
\text { TAUNTON R (QUEQUECHAN R) }\end{array}$ & U & 500.00 & 0.90 & 450.00 \\
\hline MAO 14 & $\begin{array}{l}\text { FISHERVILLE POND } \\
\text { QUINSIGAMOND } R\end{array}$ & U & 425.00 & 0.25 & 106.25 \\
\hline 03465 & $\begin{array}{l}\text { NEEDHAM (SOUTH ST) } \\
\text { CHARLES } R\end{array}$ & พo & 165.00 & 0.50 & 82.50 \\
\hline 06278 & $\begin{array}{l}\text { LAKE RIPPLE DAM } \\
\text { QUINSIGAMOND } R\end{array}$ & พо & 26.00 & 0.90 & 23.40 \\
\hline 09486 & $\begin{array}{l}\text { MOODY ST (WALTHAM) } \\
\text { CHARLES R }\end{array}$ & wo & 364.00 & 0.50 & 182.00 \\
\hline $10121 \mathrm{~A}$ & $\begin{array}{l}\text { CIRCULAR DAM } \\
\text { CHARLES R }\end{array}$ & Wo & 275.00 & 0.50 & 137.50 \\
\hline 10121B & $\begin{array}{l}\text { SILK MILL } \\
\text { CHARLES R }\end{array}$ & wo & 275.00 & 0.50 & 137.50 \\
\hline 08890 & $\begin{array}{l}\text { NEWTON UPPER FALLS } \\
\text { CHARLES } R\end{array}$ & พo & 350.00 & 0.50 & 175.00 \\
\hline 09333 & $\begin{array}{l}\text { COCHRANE } \\
\text { CHARLES R }\end{array}$ & พo & 200.00 & 0.50 & 100.00 \\
\hline 07486 & $\begin{array}{l}\text { CORDINGLY DAM } \\
\text { CHARLES R }\end{array}$ & พO & 328.00 & 0.50 & 164.00 \\
\hline 06807 & $\begin{array}{l}\text { WALTER BAKER DAM } \\
\text { NEPONSET R }\end{array}$ & พ० & 600.00 & 0.50 & 300 \\
\hline
\end{tabular}


Page No.

$03 / 21 / 95$

FERC
Number

MA013

BLACKSTONE DAM

BLACKSTONE $\mathbf{R}$

10054 MOTHER BR PWR MUSEUM MOTHER BK, NEPONSET $R$

** Subtotal **

** River Basin THAMES RIVER BASIN

MA015 QUINEBAUG $R$ POND QUINEBAUG $\mathbf{R}$

05826 SOUTH VILIAGE MILI FRENCH R

09040 GORDON

LITTLE R, FRENCH R

09255 WESTVILLE QUINEBAUG $R$

** Subtotal **

*** Total ***
HYDROPOWER RESOURCE ASSESSMENT BY RIVER BASIN

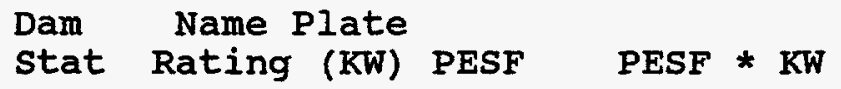

wo

$400.00 \quad 0.50$

200.00

wo

$186.00 \quad 0.50$

93.00

4779.00

2497.90

$\mathrm{U}$

$390.00 \quad 0.50$

195.00

พั

$285.00 \quad 0.90$

256.50

wo

$25.00 \quad 0.90$

22.50

พo

$1000.00 \quad 0.90$

900.00

1700.00

1374.00

325183.00

132227.90 


\section{Appendix C}

\section{Massachusetts Sites List}


Page No.

$03 / 21 / 95$

1

HYDROPOWER RESOURCE ASSESSMENT BY FERC NUMBER

\section{Plant Name/ \\ Stream}

** FERC Number 03123

STILLWATER BRIDGE

DEERFIELD $R$

** Subtotal **

** FERC Number 05057

FACTORY HOLLOW

MILI $R$, CONNECTICUT $R$

** Subtotal **

** FERC Number 05873

BARRE FALLS

WARE R, CHICOPEE $R$

** Subtotal **

** FERC Number 07917

WILLOW MILL

HOUSATONIC $R$

** Subtotal **

** FERC Number 08933

BEAR ROCK FALLS

BEAR ROCK STR, HOUSATONIC R

** Subtotal **

** FERC Number 09037

COLUMBIA MILL

HOUSATONIC $\mathbf{R}$

** Subtotal **

** FERC Number 09039

GORDON DAM

HOUSATONIC $R$ $\begin{array}{lll}\text { Dam } & \text { Name Plate } & \\ \text { ST Stat } & \text { Rating (KW) PESF } & \text { PESF * KW }\end{array}$

MA U

$7000.00 \quad 0.25$

1750.00

7000.00

1750.00

MA U

$$
52.00 \quad 0.50
$$

26.00

52.00

26.00

MA U

$550.00 \quad 0.50$

275.00

550.00

275.00

MA U

$500.00 \quad 0.10$

50.00

500.00

50.00

MA U

$130.00 \quad 0.25$

32.50

130.00

32.50

MA U

$420.00 \quad 0.10$

42.00

420.00

42.00

MA U

$380.00 \quad 0.10$

38.00 
Page No.

$03 / 21 / 95$

HYDROPOWER RESOURCE ASSESSMENT BY FERC NUMBER

Plant Name/ stream

** Subtotal **

** FERC Number 09129

OTIS

FALL R, W BR FARMINGTON $R$

** Subtotal **

** FERC Number 09606

QUEQUECHAN RIVER

TAUNTON R(QUEQUECHAN R)

** Subtotal **

** FERC Number 09616

DELANEY

SQUANNACOOK $R$

** Subtotal **

** FERC Number 09640

TEXTILE UPPER

SWIFT $R$

** Subtotal **

** FERC Number 09720

TEXTILE LOWER

SWIFT R

** Subtota1 **

** FERC Number 10032

CROCKER MILL

HOLYOKE CNL (CONN R)

** Subtotal **
Dam Name Plate

ST stat Rating (KW) PESF

PESF * KW

$$
380.00
$$

38.00

MA U

$500.00 \quad 0.10$

50.00

500.00

50.00

MA U

$500.00 \cdot 0.90$

450.00

500.00

450.00

MA U

$105.00 \quad 0.90$

94.50

105.00

94.50

MA U

$150.00 \quad 0.90$

135.00

150.00

135.00

MA U

$150.00 \quad 0.90$

135.00

150.00

135.00

MA U

$575.00 \quad 0.90$

517.50

575.00

517.50 
Page No. 3

$03 / 21 / 95$

HYDROPOWER RESOURCE ASSESSMENT BY FERC NUMBER

Plant Name/ Stream

** FERC Number MA007

I.ANCASTER MILL POND

NASHUA $R$

** Subtotal **

** FERC Number MA011

WATERTOWN

CHARLES R

** Subtotal **

** FERC Number MA012

SOUTH WATUPPA POND

QUEQUECHAN $R$

** Subtotal **

** FERC Number MA014

FISHERVILLE POND

QUINSIGAMOND $R$

** Subtotal **

** FERC Number MA015

QUINEBAUG $R$ POND

QUINEBAUG $R$

** Subtotal **

** FERC Number MA016

WEST SPRINGFIELD

WESTFIELD $R$

** Subtotal **

** FERC Number MA017

GRANVILIE

MUNN BK, LITTLE R $\begin{array}{lll}\text { Dam } & \text { Name Plate } & \\ \text { ST Stat } & \text { Rating (KW) PESF } & \text { PESF * KW }\end{array}$

MA U

$360.00 \quad 0.25$

90.00

$$
360.00
$$

90.00

MA U

$415.00 \quad 0.25$

103.75

415.00

103.75

MA U

$270.00 \quad 0.90$

243.00

270.00

243.00

MA U

$425.00 \quad 0.25$

106.25

425.00

106.25

MA U

$390.00 \quad 0.50$

195.00

390.00

195.00

MA U

$230.00 \quad 0.50$

115.00

230.00

115.00

MA U

$120.00 \quad 0.90$

108.00 
Page No.

$03 / 21 / 95$

HYDROPOWER RESOURCE ASSESSMENT BY FERC NUMBER Plant Name/
Stream

** Subtotal **

** FERC Number MAO18

THE GORGE

LITTLE R

** Subtotal **

** FERC Number MA022

HUNTINGTON

WESTFIELD $R$

** Subtotal **

** FERC Number MA025

LUDLOW (RED BRIDGE)

CHICOPEE $R$

** Subtotal **

** FERC Number MA027

TIGHE CARMODY

MANHAN $R$

** Subtotal **

** FERC Number MA031

GARDNER FALLS

DEERFIELD $R$

** Subtotal **

** FERC Number MA033

CHARLEMONT

DEERFIELD $R$

** Subtotal ** $\begin{array}{lll}\text { Dam } & \text { Name Plate } & \\ \text { ST Stat } & \text { Rating (KW) PESF } & \text { PESF * KW }\end{array}$

$$
120.00
$$

108.00

MA U

$$
490.00 \quad 0.90
$$

441.00

490.00

441.00

MA U

$6000.00 \quad 0.50$

3000.00

6000.00

3000.00

MA U

$1185.00 \quad 0.50$

592.50

1185.00

592.50

MA U

$230.00 \quad 0.50$

115.00

230.00

115.00

MA U

$1720.00 \quad 0.25$

430.00

1720.00

430.00

MA U

$20000.00 \quad 0.25$

5000.00

20000.00

5000.00 
Page No.

5

$03 / 21 / 95$

HYDROPOWER RESOURCE ASSESSMENT BY FERC NUMBER

Plant Name/ Stream

** FERC Number MA035

ZOAR

DEERFIELD $R$

** Subtotal **

** FERC Number MA037

TURNERS FALLS

CONNECTICUT R

** Subtotal **

** FERC Number MA040

ERVING

MIILERS $\mathbf{R}$

** Subtotal **

** FERC Number MA041 LAKE ROHUNTA

WILLOW BK, MILLERS R

** Subtotal **

** FERC Number MA042

TULLY

MILLERS $\mathbf{R}$

** Subtotal **

** FERC Number 01889

CABOT

CONNECTICUT $R$

** Subtotal **

** FERC Number 02801

GLENDALE

HOUSATONIC $R$ $\begin{array}{ll}\text { Dam } & \text { Name Plate } \\ \text { Stat } & \text { Rating (KW) PESF }\end{array}$

MA U

$20000.00 \quad 0.25$

5000.00

20000.00

5000.00

MA U

$66000.00 \quad 0.25$

16500.00

66000.00

16500.00

MA U

$30000.00 \quad 0.50$

15000.00

30000.00

15000.00

MA U

$$
260.00 \quad 0.25
$$

65.00

$$
260.00
$$

65.00

MA U

$20000.00 \quad 0.25$

5000.00

20000.00

5000.00

MA $W$

$23900.00 \quad 0.50$

11950.00

23900.00

11950.00

MA W

$290.00 \quad 0.10$

29.00 
Page No.

$03 / 21 / 95$

HYDROPOWER RESOURCE ASSESSMENT BY FERC NUMBER Plant Name/
Stream

** Subtotal **

** FERC Number 02985

WILLOW MILI

HOUSATONIC $\mathbf{R}$

** Subtotal **

** FERC Number 08012

HUNTS POND

MILLERS R

** Subtotal **

** FERC Number 10675

DWIGHT

CHICOPEE R

** Subtotal **

** FERC Number 10676

RED BRIDGE

CHICOPEE $R$

** Subtotal **

** FERC Number 10677

PUTTS BRIDGE

CHICOPEE $R$

** Subtotal **

** FERC Number 10678

INDIAN ORCHARD

CHICOPEE R

** Subtotal ** $\begin{array}{lll}\text { Dam } & \text { Name Plate } & \\ \text { Stat } & \text { Rating (KW) PESF } & \text { PESF * KW }\end{array}$

290.00

29.00

MA W

$360.00 \quad 0.10$

36.00

360.00

36.00

MA W

$200.00 \quad 0.50$

100.00

200.00

100.00

MA W

$210.00 \quad 0.90$

189.00

210.00

189.00

MA W

$695.00 \quad 0.90$

625.50

695.00

625.50

MA W

$370.00 \quad 0.75$

277.50

370.00

277.50

MA W

$430.00 \quad 0.75$

322.50

430.00

322.50 
Page No.

7

$03 / 21 / 95$

HYDROPOWER RESOURCE ASSESSMENT BY FERC NUMBER

Plant Name/

stream

** FERC Number 10688

COSGROVE

COSGROVE AQUE (WACHUSETT RES)

** Subtotal **

** FERC Number MA020

RUSSELL

WESTFIELD $R$

** Subtotal **

** FERC Number MA026

QUABBIN-WINDSOR

SWIFT $R$

** Subtotal **

** FERC Number MA029

MARTIN MACH CO DAM

SAWMIIL $\mathbf{R}$

** Subtotal **

** FERC Number 03103

STEVENS

LITTLE R

** Subtotal **

** FERC Number 03423

BIRCH HILL

MILLERS $\mathrm{R}$

** Subtotal **

** FERC Number 03465

NEEDHAM (SOUTH ST)

CHARLES $R$
Dam Name Plate

ST Stat Rating (KW) PESF

PESF * KW

MA W

$1300.00 \quad 0.50$

650.00

1300.00

650.00

MA W

$130.00 \quad 0.75$

97.50

130.00

97.50

MA $W$

$165.00 \quad 0.50$

82.50

165.00

82.50

MA W

$125.00 \quad 0.90$

112.50

125.00

112.50

MA Wo

$500.00 \quad 0.75$

375.00

500.00

375.00

MA พ०

$670.00 \quad 0.75$

502.50

670.00

502.50

MA WO

$165.00 \quad 0.50$

82.50 
Page No.

$03 / 21 / 95$

8

HYDROPOWER RESOURCE ASSESSMENT BY FERC NUMBER

Plant Name/
Stream

** Subtotal **

** FERC Number 04809

TOWNSEND HARBOR

SQUANNACOOK $R$

** Subtotal **

** FERC Number 05462

LAKE WARNER

MILI R, CONNECTICUT R

** Subtotal **

** FERC Number 05666A

GREEN TWO (MILL 2)

GREEN R,DEERFIELD $R$

** Subtotal **

** FERC Number 05666B

GREEN ONE (MILL 1)

GREEN R, DEERFIELD $R$

** Subtotal **

** FERC Number 05826

SOUTH VILLAGE MIIL

FRENCH R

** Subtotal **

** FERC Number 06096

NEW HOME SOUTH

MILIERS R

** Subtotal ** $\begin{array}{lll}\text { Dam } & \text { Name plate } & \\ \text { ST Stat } & \text { Rating (KW) PESF } & \text { PESF * KW }\end{array}$

165.00

82.50

MA Wo

$250.00 \quad 0.50$

125.00

250.00

125.00

MA Wo

$30.00 \quad 0.75$

$22 \cdot 50$

30.00

22.50

MA พO

$106.00 \quad 0.50$

53.00

106.00

53.00

MA Wo

$71.00 \quad 0.50$

35.50

71.00

35.50

MA WO

$285.00 \quad 0.90$

256.50

285.00

256.50

MA Wo

$250.00 \quad 0.75$

187.50

250.00

187.50 
Page No.

$03 / 21 / 95$

9

HYDROPOWER RESOURCE ASSESSMENT BY FERC NUMBER

Plant Name/

stream

** FERC Number 06278

LAKE RIPPLE DAM

QUINSIGAMOND $R$

** Subtotal **

** FERC Number 06556

GLENALLAN MILLS

N BR MILLERS R

** Subtotal **

** FERC Number 06807

WALTER BAKER DAM

NEPONSET $R$

** Subtotal **

** FERC Number 06966

BRASSWORKS

MIIL $R$

** Subtotal **

FERC Number 07367

WATERSHOPS

MILL R

** Subtotal **

** FERC Number 07486

CORDINGLY DAM

CHARLES $\mathbf{R}$

** Subtotal **

** FERC Number 08234A

MILLERS ONE

MILLERS $\mathrm{R}$
Dam Name Plate
STat Rating (KW)

Rating (KW) PESF

PESF * KW

MA Wo

$26.00 \quad 0.90$

23.40

26.00

23.40

MA พo

$79.00 \quad 0.75$

59.25

79.00

59.25

MA Wo

$600.00 \quad 0.50$

300.00

600.00

300.00

MA Wo

$75.00 \quad 0.75$

56.25

75.00

56.25

MA WO

$100.00 \quad 0.75$

75.00

100.00

75.00

MA WO

$328.00 \quad 0.50$

164.00

328.00

164.00

MA WO

$1090.00 \quad 0.50$

545.00 
Page No. 10

$03 / 21 / 95$

HYDROPOWER RESOURCE ASSESSMENT BY FERC NUMBER Plant Name/
Stream

** Subtotal **

** FERC Number 08234B

MILLERS TWO

MILLERS $R$

** Subtotal **

** FERC Number $08234 \mathrm{C}$

MILLERS THREE

MILLERS $\mathbf{R}$

** Subtotal **

** FERC Number 08234D

MILLERS FOUR

MILLERS R

** Subtotal **

** FERC Number 08234E

MILLERS FIVE

MILLERS $\mathrm{R}$

** Subtotal **

** FERC Number 08293

KENNEY POND

OTTER R,MILLERS $R$

** Subtotal **

** FERC Number 08350

LITTLEVILLE

M BR WESTFIELD $R$

** Subtotal ** $\begin{array}{lll}\text { Dam } & \text { Name Plate } & \\ \text { Stat } & \text { Rating (KW) PESF } & \text { PESF * KW }\end{array}$

1090.00

545.00

MA Wo

$1950.00 \quad 0.50$

975.00

1950.00

975.00

MA Wo

$1090.00 \quad 0.50$

545.00

1090.00

545.00

MA Wo

$800.00 \quad 0.50$

400.00

800.00

400.00

MA Wo

$800.00 \quad 0.50$

400.00

800.00

400.00

MA WO

$100.00 \quad 0.50$

50.00

100.00

50.00

MA WO

$1060.00 \quad 0.50$

530.00

1060.00

530.00 
Page No.

$03 / 21 / 95$

HYDROPOWER RESOURCE ASSESSMENT BY FERC NUMBER

Plant Name/
Stream

** FERC Number 08424

BILLERICA

CONCORD $\mathbf{R}$

** Subtotal **

** FERC Number 08449

BERNARDSTON GRAIN MILL

FAIL $\mathbf{R}$

** Subtotal **

** FERC Number 08641

BALIUARDVALE

SHAWSHEEN $R$

** Subtotal **

** FERC Number 08652

PLEASANT STREET

BEAVER BK, MERRIMACK R

** Subtotal **

** FERC Number 08653

ANDOVER

SHAWSHEEN $\mathbf{R}$

** Subtotal **

** FERC Number 08890

NEWTON UPPER FALLS

CHARLES $R$

** Subtotal **

** FERC Number 09040

GORDON

LITTLE R, FRENCH R $\begin{array}{lll}\text { Dam } & \text { Name Plate } & \\ \text { ST } & \text { Rating (KW) PESF } & \text { PESF * KW }\end{array}$

MA WO

$200.00 \quad 0.50$

100.00

200.00

100.00

MA WO

$80.00 \quad 0.90$

72.00

80.00

72.00

MA WO

$40.00 \quad 0.90$

36.00

40.00

36.00

MA พO

$65.00 \quad 0.75$

48.75

65.00

48.75

MA Wo

$50.00 \quad 0.90$

45.00

50.00

45.00

MA Wo

$350.00 \quad 0.50$

175.00

350.00

175.00

MA Wo

$25.00 \quad 0.90$

22.50 
Plant Name/ stream

** Subtotal **

** FERC Number 09066 CONWAY

SOUTH $R, D E E R F I E L D ~ R$

** Subtotal **

** FERC Number 09081

CENTER POND

E BR HOUSATONIC $R$

** Subtotal **

** FERC Number 09082

DALTON NO 1

E BR HOUSATONIC $R$

** Subtotal **

** FERC Number 09083

DALTON NO 6

$E$ BR HOUSATONIC $R$

** Subtotal **

** FERC Number 09255

WESTVILLE

QUINEBAUG $\mathbf{R}$

** Subtotal **

** FERC Number 09276

COOKS

MILL R

** Subtotal **
Dam
ST Stat Plate
Rating (KW) PESF

$$
25.00
$$

22.50

MA Wo

$350.00 \quad 0.50$

175.00

350.00

175.00

MA WO

$130.00 \quad 0.75$

97.50

130.00

97.50

MA Wo

$100.00 \quad 0.50$

50.00

100.00

50.00

MA พO

$140.00 \quad 0.50$

70.00

140.00

70.00

MA Wo

$1000.00 \quad 0.90$

900.00

1000.00

900.00

MA Wo

$180.00 \quad 0.50$

90.00

180.00

90.00 
Page No. 13

$03 / 21 / 95$

HYDROPOWER RESOURCE ASSESSMENT BY FERC NUMBER

Plant Name/

stream

** FERC Number

STEARNS

SUDBURY R

** Subtotal **

** FERC Number 09288

SAXONVIILE

SUDBURY $R$

** Subtotal **

** FERC Number 09329

IEEDS

MILL R

** Subtotal **

** FERC Number 09330 NONOTUCKET STREET

MILL $\mathbf{R}$

** Subtotal **

** FERC Number 09331

SPRING STREET

MILL R

** Subtotal **

** FERC Number 09332

PARADISE

MILL $R$

** Subtotal **

** FERC Number 09333

COCHRANE

CHARLES R
Dam Name Plate

ST stat Rating (KW) PESF

PESF * KW

MA WO

$60.00 \quad 0.50$

30.00

60.00

30.00

MA Wo

$150.00 \quad 0.50$

75.00

150.00

75.00

MA พ०

$\begin{array}{lll}75.00 & 0.75\end{array}$

56.25

75.00

56.25

MA พO

$75.00 \quad 0.75$

56.25

75.00

56.25

MA Wo

$100.00 \quad 0.90$

90.00

100.00

90.00

MA wo

$75.00 \quad 0.50$

37.50

75.00

37.50

MA Wo

$200.00 \quad 0.50$

100.00 


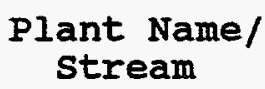

** Subtotal **

** FERC Number 09432

MANHAN

MANHAN $\mathbf{R}$

** Subtotal **

** FERC Number 09486 MOODY ST (WALTHAM)

CHARLES $R$

** Subtotal **

** FERC Number 09615 THREE RIVERS

CHICOPEE $R$

** Subtotal **

** FERC Number 09895 KNIGHTVILLE WESTFIELD $R$

** Subtotal **

** FERC Number 09978 IAAKE GARDNER POWWOW $R$, MERRIMACK $R$

** Subtotal **

** FERC Number 10012

WASHINGTON STREET

ASSABET $R$

** Subtotal **

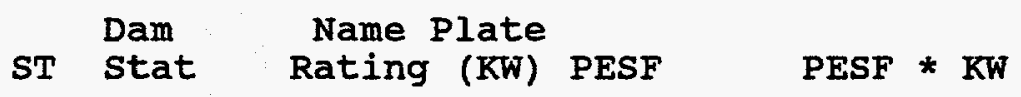

MA พO

$142.00 \quad 0.90$

127.80

142.00

127.80

MA WO

$364.00 \quad 0.50$

182.00

364.00

182.00

MA WO

$2360.00 \quad 0.50$

1180.00

2360.00

1180.00

MA พั

$3000.00 \quad 0.50$

1500.00

3000.00

1500.00

MA WO

$120.00 \quad 0.75$

90.00

120.00

90.00

MA WO

$100.00 \quad 0.50$

50.00

100.00

50.00 
Page No.

$03 / 21 / 95$

HYDROPOWER RESOURCE ASSESSMENT BY FERC NUMBER

Plant Name/ Stream

** FERC Number 10054

MOTHER BK PWR MUSEUM

MOTHER BK, NEPONSET R

** Subtotal **

** FERC Number 10121A

CIRCULAR DAM

CHARLES R

** Subtotal **

** FERC Number 10121B

SILK MILL

CHARLES $\mathbf{R}$

** Subtotal **

** FERC Number 10177

SHAKER MILL

WILIIAMS $R$

** Subtotal **

** FERC Number 10338

WEST GROTON

SQUANNACOOK $R$

** Subtotal **

** FERC Number 10563

GEO MAC MEMORIAL

WAMESIT CNL

** Subtotal **

** FERC Number 10580

POW WOW RIVER

POWWOW $R$, MERRIMACK $R$ $\begin{array}{lll}\text { Dam } & \text { Name Plate } & \\ \text { ST Stat } & \text { Rating (KW) PESF } & \text { PESF * KW }\end{array}$

MA WO

$186.00 \quad 0.50$

93.00

186.00

93.00

MA Wo

$275.00 \quad 0.50$

137.50

275.00

137.50

MA WO

$275.00 \quad 0.50$

137.50

275.00

137.50

MA Wo

$30.00 \quad 0.50$

15.00

30.00

15.00

MA Wo

$210.00 \quad 0.90$

189.00

210.00

189.00

MA Wo

$376.00 \quad 0.90$

338.40

376.00

338.40

MA พo

$300.00 \quad 0.75$

225.00 


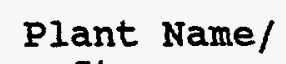

$$
500.00
$$

MA Wo

$$
200.00 \quad 0.75
$$

$$
200.00
$$

MA Wo

$$
50.00 \quad 0.75
$$

$$
50.00
$$

MA wo

$1200.00 \quad 0.10$

120.00

1200.00

120.00

MA Wo

$1300.00 \quad 0.50$

650.00

1300.00

650.00 
Page No.

$03 / 21 / 95$
17

HYDROPOWER RESOURCE ASSESSMENT BY FERC NUMBER
Plant Name/ Stream

\section{Dam \\ ST Stat}

MA Wo

** FERC Number 11111

CROCKER BURBANK MILL 6

NASHUA $R$

** Subtotal **

** FERC Number 11166

SNOWS MILL POND

WHITMAN $R$, NASHUA $R$

** Subtotal **

** FERC Number 11306

ECLIPSE DAM

NBR HOOSIC RIVER

** Subtotal **

** FERC Number 11337

WHITNEY POND \#1

MILLERS $R$

** Subtotal **

** FERC Number MA001 SUDBURY

STONY BK, SUDBURY R

** Subtotal **

** FERC Number MAOO2

OLD GUARD LOCKS

MERRIMACK CNL(MERR R)

** Subtotal **

** FERC Number MA003

SWAMP LOCKS

MERRIMACK CNL(MERR R)

MA Wo

MA Wo

MA wo

MA พo

MA Wo

MA Wo
Name Plate

Rating (KW) PESF

PESF * KW
75.00

$$
350.00 \quad 0.90
$$

$$
350.00
$$

315.00

$600.00 \quad 0.75$

450.00

$$
600.00
$$

450.00

$550.00 \quad 0.50$

275.00

$$
550.00
$$

275.00

$265.00 \quad 0.50$

132.50

265.00

132.50

$24600.00 \quad 0.50$

12300.00

24600.00

12300.00

$17900.00 \quad 0.50$

8950.00 
HYDROPOWER RESOURCE ASSESSMENT BY FERC NUMBER

Plant Name/ stream

** Subtotal **

** FERC Number MA004

PAWTUCKET (4)

FOUR CANALS (MERR R)

** Subtotal **

** FERC Number MA005

NORTH CANAL LOCKS

MERRIMACK CNL (MERR R)

** Subtotal **

** FERC Number MA006

PEPPERELL

NASHUA $R$

** Subtotal **

** FERC Number MA008

WACHUSETT

$S$ BR NASHUA $R$

** Subtotal **

** FERC Number MAO09

MACTAGGARTS POND

PHILLIPS BK, $N$ NASHUA $R$

** Subtotal **

** FERC Number MA010

FITCHBURG

N NASHUA $R$

** Subtotal ** $\begin{array}{lll}\text { Dam } & \text { Name Plate } & \\ \text { Stat } & \text { Rating (KW) PESF } & \text { PESF * KW }\end{array}$

17900.00

8950.00

MA Wo

$906.00 \quad 0.50$

453.00

906.00

453.00

MA WO

$33600.00 \quad 0.50$

16800.00

33600.00

16800.00

MA WO

$470.00 \quad 0.50$

235.00

470.00

235.00

MA WO

$1070.00 \quad 0.50$

535.00

1070.00

535.00

MA WO

$250.00 \quad 0.75$

187.50

250.00

187.50

MA Wo

$250.00 \quad 0.75$

187.50

250.00

187.50 
Page No.

19

$03 / 21 / 95$

HYDROPOWER RESOURCE ASSESSMENT BY FERC NUMBER

Plant Name/ stream

** FERC Number MA013

BLACKSTONE DAM

BLACKSTONE R

** Subtotal **

** FERC Number MA019

WORONCO (STRATMORE)

WESTFIELD $R$

** Subtotal **

** FERC Number MA021

RUSSEIL (CRESCENT MILLS)

WESTFIELD $R$

** Subtotal **

** FERC Number MA023

INDIAN ORCHARD

CHICOPEE $\mathbf{R}$

** subtotal **

** FERC Number MA024

PUTTS BRIDGE

CHICOPEE $R$

** Subtotal **

** FERC Number MA028

ROARING BROOK DAM

ROARING BK, MILL R

** Subtotal **

** FERC Number MA030

DENISON DAM

GREEN R,DEERFIELD $R$ $\begin{array}{ll}\text { Dam } & \text { Name Plate } \\ \text { Stat } & \text { Rating (KW) PESF }\end{array}$

MA wo

$400.00 \quad 0.50$

200.00

400.00

200.00

MA Wo

$390.00 \quad 0.75$

292.50

390.00

292.50

MA Wo

$1125.00 \quad 0.75$

843.75

1125.00

843.75

MA Wo

$785.00 \quad 0.75$

588.75

785.00

588.75

MA Wo

$2345.00 \quad 0.75$

1758.75

2345.00

1758.75

MA Wo

$52.00 \quad 0.90$

46.80

52.00

46.80

MA พo

$115.00 \quad 0.50$

57.50 
Page No. 20

$03 / 21 / 95$

HYDROPOWER RESOURCE ASSESSMENT BY FERC NUMBER

\section{Plant Name/ Stream}

** Subtotal **

** FERC Number MA032

DEERFIELD 3

DEERFIELD $\mathbf{R}$

** Subtotal **

* * FERC Number MA034

MILL BROOK

MILL BK, DEERFIELD $R$

** Subtotal **

** FERC Number MA036

SHERMAN

DEERFIELD $\mathbf{R}$

** Subtotal **

** FERC Number MA038 BOOK BINDERY POND

MILLERS $\mathbf{R}$

** Subtotal **

** FERC Number MA039

MILIERS FALLS ONE

MILLERS $\mathbf{R}$

** Subtotal **

** FERC Number MA043

VALIEY MILL

HOUSATONIC $\mathbf{R}$

** Subtotal **

*** Total *** $\begin{array}{ll}\text { Dam } & \text { Name Plate } \\ \text { ST stat } & \text { Rating (KW) PESF }\end{array}$

115.00

57.50

MA WO

$1135.00 \quad 0.50$

567.50

1135.00

567.50

MA WO

$100.00 \quad 0.50$

50.00

100.00

50.00

MA WO

$3020.00 \quad 0.50$

1510.00

3020.00

1510.00

MA WO

$1000.00 \quad 0.75$

750.00

1000.00

750.00

MA WO

$500.00 \quad 0.75$

375.00

500.00

375.00

MA WO

$375.00 \quad 0.10$

37.50

375.00

37.50

325183.00

132227.90 


\section{Appendix D}

Individual Resource Database List

D-1 
R E S U U C E D A T A B A S I I S T I N G

DATE: $06 / 27 / 95$

PAGE NO: 1

FERC

Number

Plant Name

Stream

State

Name

01889 CABOT

CONNECTICUT $\mathbf{R}$

MA

county Name

FRANKLIN

Class

Owner Name

P WESTERN MASS ELECTRIC CO

Name Plate

Rating (KW).

PESF

PESF*KW

Annual Energy

23900.00

$0.50 \quad 11950.00$

Rating (MWh)

$$
46300.00
$$

$\begin{array}{ccccc}\text { Unit } & \begin{array}{l}\text { Plant } \\ \text { Type }\end{array} & \begin{array}{l}\text { Project } \\ \text { Status }\end{array} & \begin{array}{l}\text { Dam } \\ \text { Status }\end{array} & \begin{array}{l}\text { Latitude } \\ \text { Iongitude }\end{array} \\ \text { C } & \text { ROR } & \text { MO } & \text { W } & \end{array}$

Factor

Exist Prob

Factor

Exist Prob

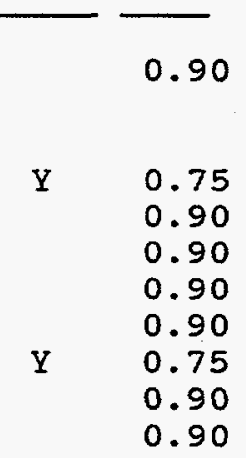

Wildlife Value Threatened/Endangered Fish Threatened/Endangered Wilalife Federal Land code 103 Federal Iand code 104 Federal Land Code 105 Federal Land Code 106 Federal Iand code 107 Federal Land Code 108 Federal Land Code 198
23150

PESF Annual Energy Rating (MWh)

4237.00

7233.00
0.90
Wild/Scenic Protection

Wild/Scenic Tributary or Upstream/Downstream cultural Value

Fish Presence Value

Geologic Value

Historic Value

other Value

Recreation Value

Scenic Value
0.90

0.90

0.90

0.90

0.90

0.90

0.90

0.90

0.90

0.90 
DATE: $06 / 27 / 95$

PAGE NO: 2

FERC

Number

02801
Plant Name

GLENDALE

County Name

BERKSHIRE
State

Name

HOUSATONIC $\mathbf{R}$

MA

$$
\text { River Basin }
$$

HOUSATONIC RIVER BASIN

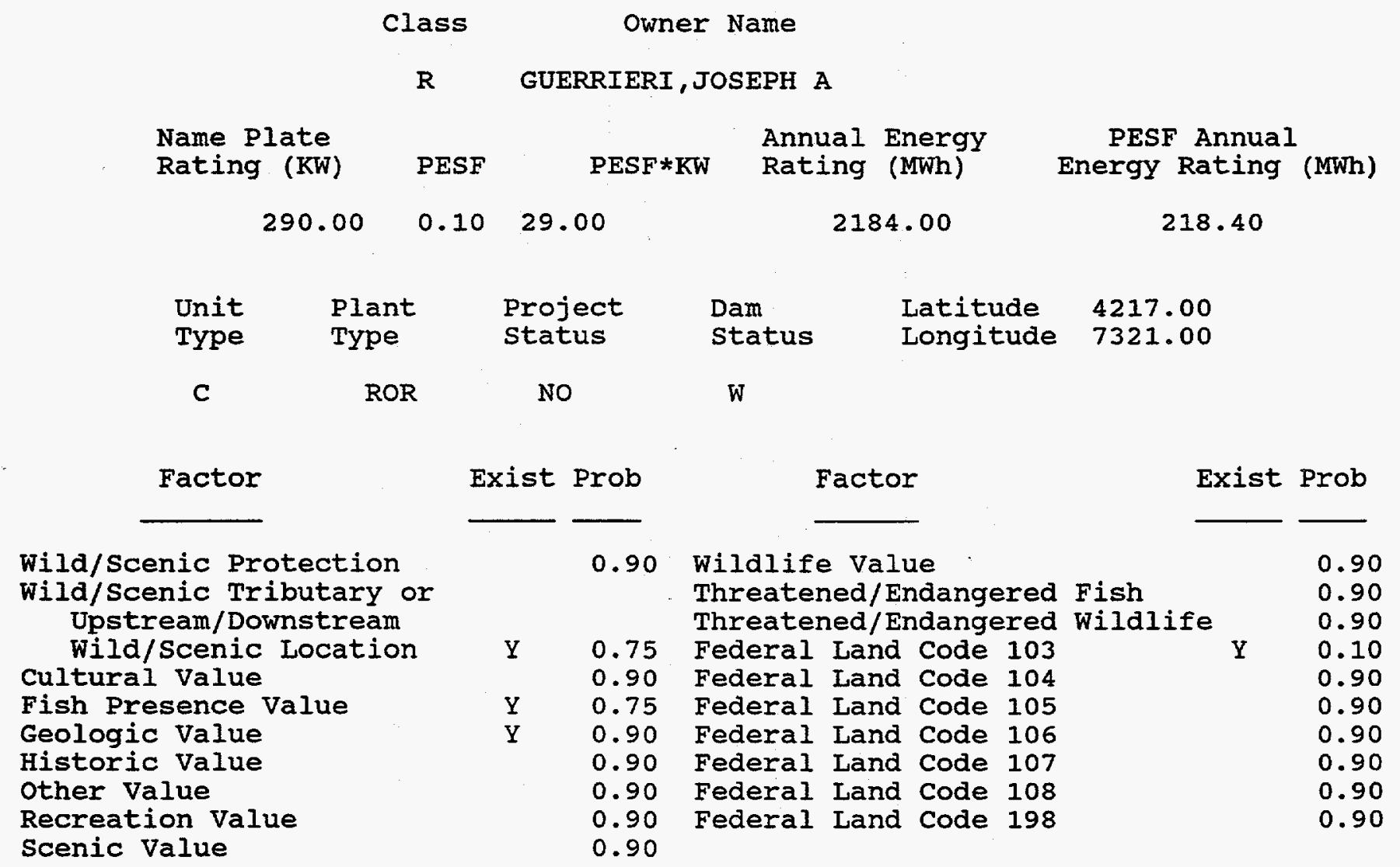




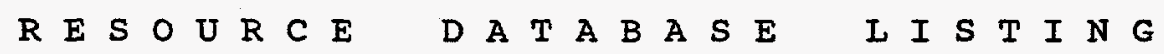

DATE: $06 / 27 / 95$

PAGE NO: 3

FERC

Number

Plant Name

Stream

State

02985 WILLOW MILI

HOUSATONIC $\mathbf{R}$

Name

02985

County Name

River Basin

BERKSHIRE

HOUSATONIC RIVER BASIN

$\begin{array}{cc}\text { class } & \text { Owner Name } \\ \text { I } & \text { MEAD CORP }\end{array}$

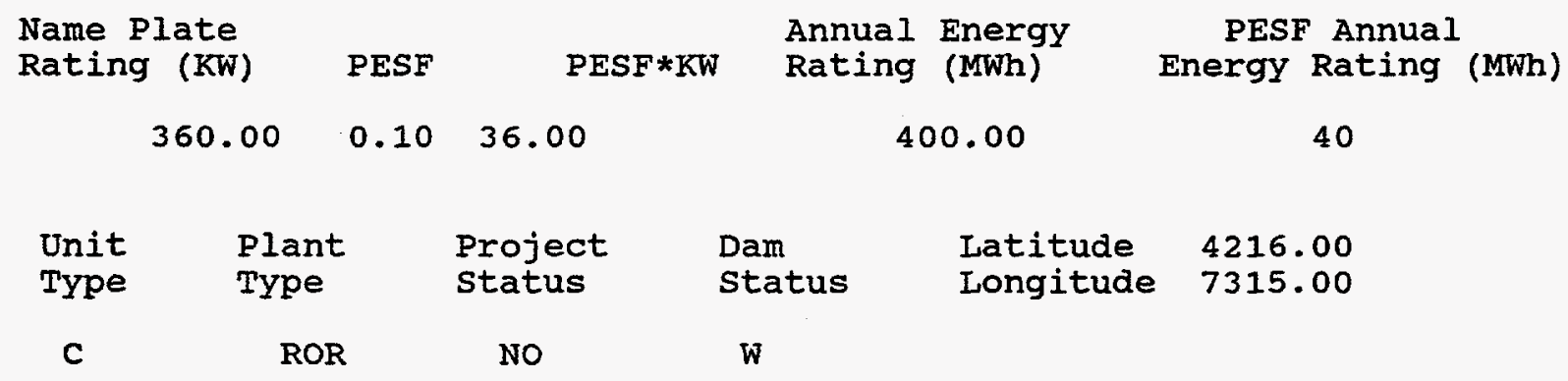

\section{Factor}

wild/Scenic Protection

Wild/Scenic Tributary or Upstream/Downstream wild/Scenic Location Cultural value

Fish Presence Value

Geologic Value

Historic Value

other Value

Recreation Value

Scenic Value
Exist Prob

Factor

Exist Prob

\begin{tabular}{l}
\hline \\
$Y$ \\
$Y$ \\
$Y$
\end{tabular}

\subsection{0}

Wildlife Value

Threatened/Endangered Fish Threatened/Endangered Wildlife

0.75 Federal Land code 103

0.90 Federal Land code 104

0.75 Federal Land code 105

0.90 Federal Land code 106

0.90 Federal Land Code 107

0.90 Federal Land code 108

0.90 Federal Land code 198

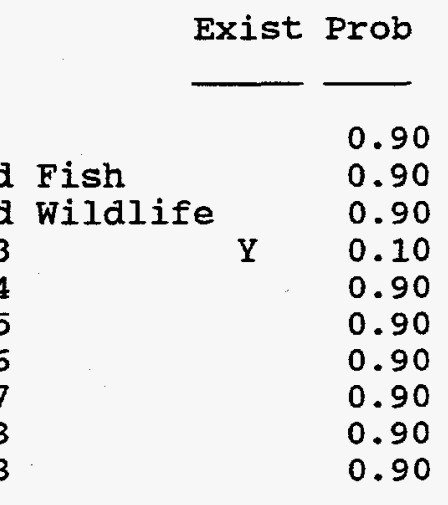


R E S O U R C E

DATE: $06 / 27 / 95$

FERC

Number

Plant Name

03103 STEVENS

county Name

HAMPDEN

class

Owner Name

M

WESTFIELD, CITY OF

Name Plate

Rating ( $\mathrm{KW}$ )

PESF

500.00

0.75375 .00

PESF*KW

Annual Energy

Rating (MWh)

913.00

Latitude

Longitude
State Name

MA

$\begin{array}{cccll}\text { Unit } & \text { Plant } & \text { Project } \\ \text { Type } & \text { Type } & \text { Status } & \begin{array}{l}\text { Dam } \\ \text { Status }\end{array} & \begin{array}{l}\text { Latitude } \\ \text { Longitude }\end{array} \\ \text { C } & \text { ROR } & \text { XX } & \text { wo } & \end{array}$

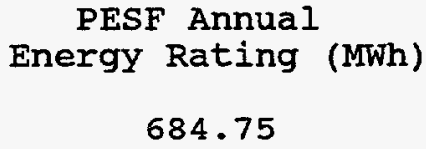

PESF Annual

684.75
0.00

0.00
Factor

Wild/Scenic Protection

Wild/Scenic Tributary or Upstream/ Downstream wild/Scenic Location Cultural value

Fish Presence Value

Geologic Value

Historic Value

other Value

Recreation Value

Scenic Value
Exist Prob

$\mathbf{Y}$

0.75

0.90

0.90

0.90

0.90

0.90

0.90

0.90
Factor

Exist Prob

wildife Value

Threatened/Endangered Fish

Threatened/Endangered Wildlife

0.90

0.90

0.90

0.90

0.90

0.90

Federal Land Code 104

Federal Land Code 106

Federal Land code 107

0.90

0.90

0.90

Federal Land Code 108

0.90 
RE SOU R C E D A T A B A S E I I S T I N G

DATE: $06 / 27 / 95$

PAGE NO: 5

FERC

Number

03123

STILLWATER BRIDGE

County Name

FRANKLIN

Plant Name
M MASS MUN WHOLESALE ELEC CO

Name Plate

Rating (KW)
7000.00

Annual Energy

Rating (MWh)

36000.00

Latitude

Longitude

4231.00

Unit

Plant

Project status

$\mathrm{XX}$

Status

U
C

ROR state

Name

MA
Exist Prob

Factor

wild/Scenic Protection

wild/Scenic Tributary or Upstream/Downstream wild/Scenic Location Cultural value

Fish Presence Value

Geologic Value

Historic Value

other Value

Recreation Value

Scenic Value

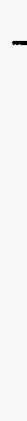

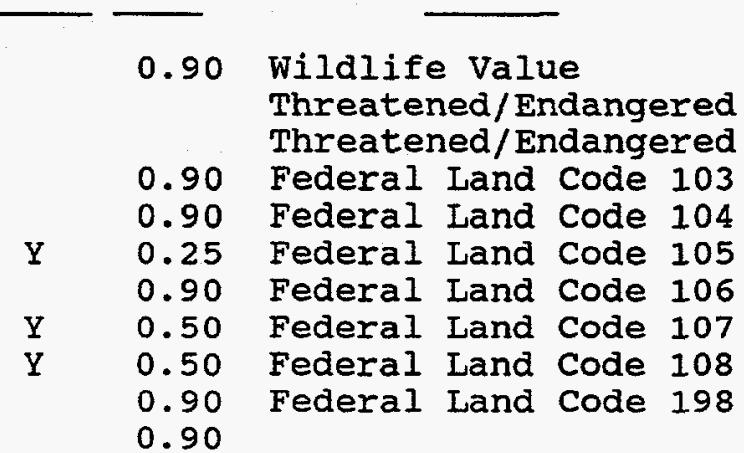

Exist Prob

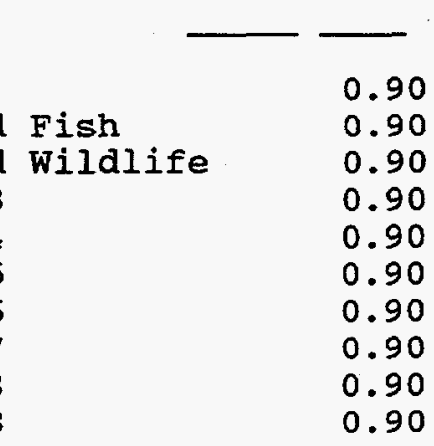


FERC

Number

Plant Name
BIRCH HILL

County Name

WORCESTER
State

Name

MILLERS $\mathrm{R}$

MA

River Basin

CONNECTICUT RIVER BASIN

Class Owner Name

R WATER POWER DEV CORP

$\begin{array}{lrrcr}\begin{array}{l}\text { Name Plate } \\ \text { Rating (KW) }\end{array} & \text { PESF } & \text { PESF*KW } & \begin{array}{l}\text { Annual Energy } \\ \text { Rating (MWh) }\end{array} & \begin{array}{c}\text { PESF Annual } \\ \text { Energy Rating (MWh) }\end{array} \\ 670.00 & 0.75 & 502.50 & 2700.00 & 2025\end{array}$

Unit Plant Project Dam Latitude 4238.00

Type Type Status Status Longitude 7207.00

C

ROR

XX

WO

\author{
Factor \\ Wild/Scenic Protection \\ wild/Scenic Tributary or \\ Upstream/Downstream \\ wild/Scenic Location \\ Cultural Value \\ Fish Presence Value \\ Geologic Value \\ Historic Value \\ other Value \\ Recreation Value \\ Scenic Value
}

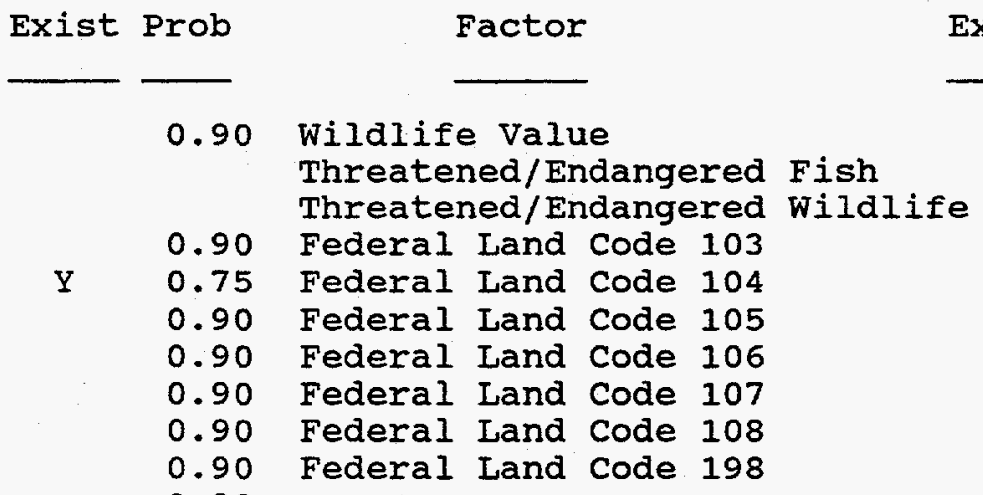

0.90

0.90

0.90

0.90

0.90

0.90

0.90 
RES S U R C E D A T A B A S E I I S T I N G

DATE: $06 / 27 / 95$

PAGE NO: 7

FERC

Number

Plant Name

stream

state

03465 NEEDHAM (SOUTH ST)

CHARLES R

Name

county Name

River Basin

NORFOLK

MINOR RIVER BASINS

$\begin{array}{cr}\text { Class } & \text { Owner Name } \\ \text { R } & \text { KNOTT, JAMES M }\end{array}$

Name Plate

PESF

Annual Energy

Rating (KW)

$0.50 \quad 82.50$

PESF*KW

Rating (MWh)

PESF Annual

165.00

595.00

297.50

$\begin{array}{cccll}\text { Unit } & \text { Plant } & \text { Project } & \text { Dam } & \text { Latitude } \\ \text { Type } & \text { Type } & \text { Status } & \text { Status } & \text { Longitude } 7112.00 \\ \text { C } & \text { ROR } & \text { XX } & \text { wo } & \end{array}$

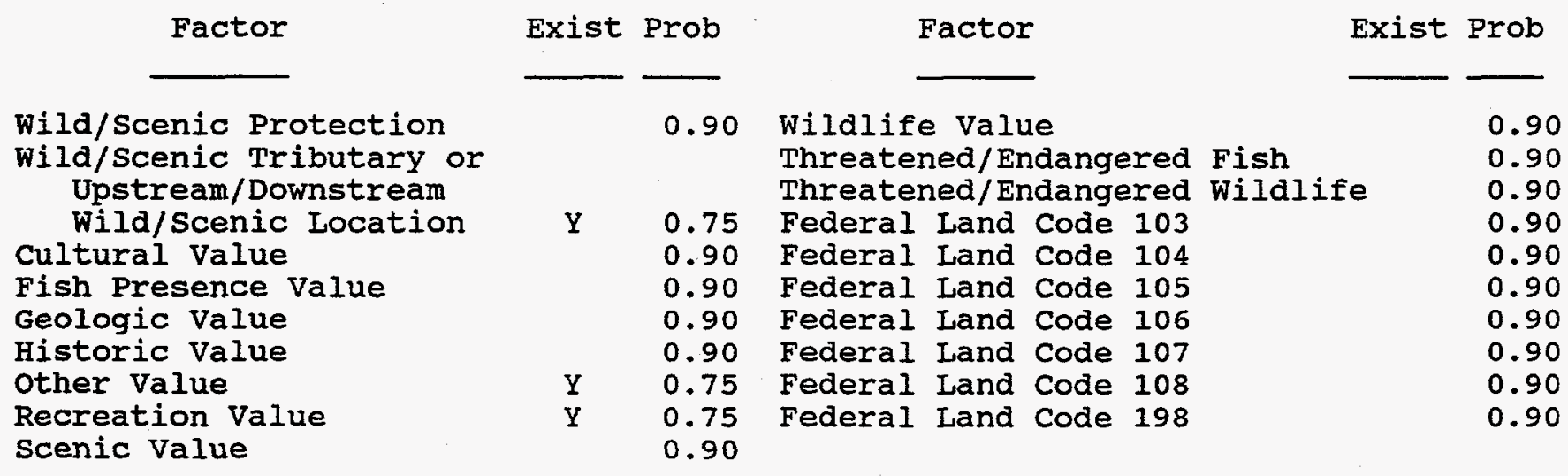


FERC

Number

Plant Name

04809 TOWNSEND HARBOR

county Name

MIDDLESEX stream

SQUANNACOOK $R$

River Basin

MERRIMACK RIVER BASIN state

Name

MA

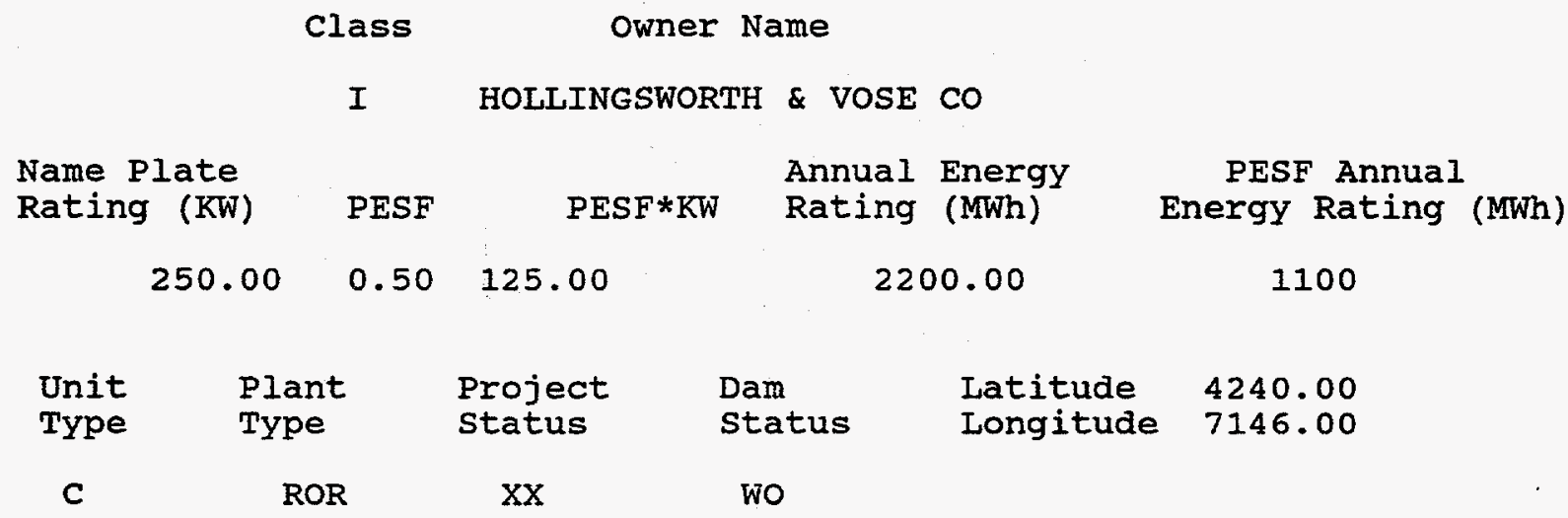

Factor

Exist Prob

wild/Scenic Protection

wild/Scenic Tributary or Upstream/Downstream wild/Scenic Location Cultural value

Fish Presence Value

Geologic Value

Historic Value

Other Value

Recreation Value

scenic Value
Factor

Exist Prob

$\begin{array}{ll} & \\ & \\ & \\ & \\ & \\ & 0.90 \\ & 0.75 \\ & 0.90 \\ 0.90 \\ 0.90 \\ 0.90 \\ 0.90 \\ 0.90 \\ 0.90\end{array}$

Wildlife value Threatened/Endangered Fish Threatened/Endangered Wildlife $Y$

0.75 Federal Land Code 103 Federal Land Code 104

Federal Land Code 105

Federal Land code 106

Federal Land Code 107

Federal Land code 108

Federal Land code 198

0.75

0.75

0.90

0.90

0.90

0.90

0.90

0.90

0.90 

R E S O U R C E
D A T A B A S E
I I S T I N G

DATE: $06 / 27 / 95$

PAGE NO: 9

FERC

Number

05057
Plant Name

FACTORY HOLLOW

county Name

HAMPSHIRE
State

Name

MA
MILL $R$, CONNECTICUT $R$

River Basin

CONNECTICUT RIVER BASIN

$$
\begin{array}{cr}
\text { Class } & \text { Owner Name } \\
\text { M } & \text { AMHERST, TOWN OF }
\end{array}
$$

Name Plate Rating (KW)

52.00

PESF

\begin{abstract}
PESF*KW
\end{abstract}
Annual Energy Rating (MWh)

210.00

Latitude Longitude
PESF Annual Energy Rating (MWh)

105

$\begin{array}{ccccc}\text { Unit } & \begin{array}{l}\text { Plant } \\ \text { Type }\end{array} & \begin{array}{l}\text { Project } \\ \text { Status }\end{array} & \begin{array}{l}\text { Dam } \\ \text { Status }\end{array} & \begin{array}{l}\text { Latitude } \\ \text { Longitude }\end{array} \\ \text { C } & \text { ROR } & \mathrm{XX} & \mathrm{U} & \end{array}$

Factor

Wild/Scenic Protection

wild/Scenic Tributary or Upstream/Downstream wild/Scenic Location cultural value Fish Presence Value Geologic Value Historic Value other Value Recreation Value Scenic Value

\section{Exist Prob}

Factor

0.90 Wildlife Value Threatened/Endangered Fish Threatened/Endangered Wildilfe 4225.00 7231.00

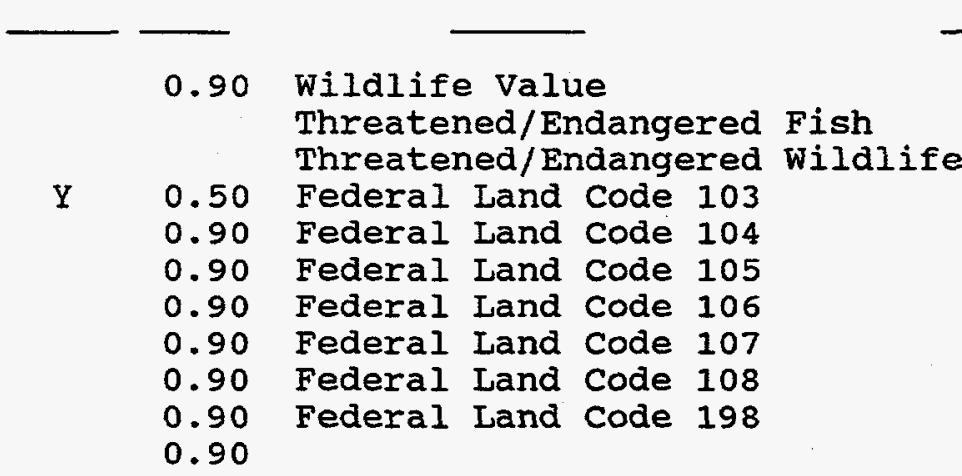

Exist Prob 
FERC

Number

Plant Name

05462 LAKE WARNER

county Name

HAMPSHIRE
State

Name

MA
MIIL $R$, CONNECTICUT $R$

River Basin

CONNECTICUT RIVER BASIN class Owner Name

R KLIMOSKI, LYDIA A
PESF Annual Energy Rating (MWh)

Annual Energy Rating (MWh)

171.00

128.25

$\begin{array}{ccclll}\text { Unit } & \text { Plant } & \text { Project } & \text { Dam } & \text { Latitude } & 4225.00 \\ \text { Type } & \text { Type } & \text { Status } & \text { Status } & \text { Longitude } & 7233.00 \\ \text { C } & \text { ROR } & \text { XX } & \text { wo } & \end{array}$

Factor

wild/Scenic Protection

wild/Scenic Tributary or Upstream/Downstream wild/scenic Location Cultural value Fish Presence Value Geologic Value Historic Value other Value Recreation Value Scenic Value
Exist Prob

Factor

Exist Prob

0.90

Wildlife Value

Threatened/Endangered Fish

Threatened/Endangered Wildlife

Y 0.75 Federal Land code 103

0.90 Federal Land Code 104

0.90 Federal Land Code 105

0.90 Federal Land Code 106

0.90 Federal Iand Code 107

0.90 Federal Land Code 108

0.90 Federal Land Code 198

0.90
0.90

0.90

0.90

0.90

0.90

0.90

0.90

0.90

0.90

0.90 
RESOURCE DA T A B A S E L I S T I N G

DATE: $06 / 27 / 95$

PAGE NO: 11

FERC

Number

Plant Name

05666A GREEN TWO(MILI 2)

GREEN $R$, DEERFIELD $R$

State

Name

county Name FRANKLIN

River Basin

CONNECTICUT RIVER BASIN

$\begin{array}{cc}\text { Class } & \text { Owner Name } \\ \text { M } & \text { GREENFIELD, TOWN OF }\end{array}$

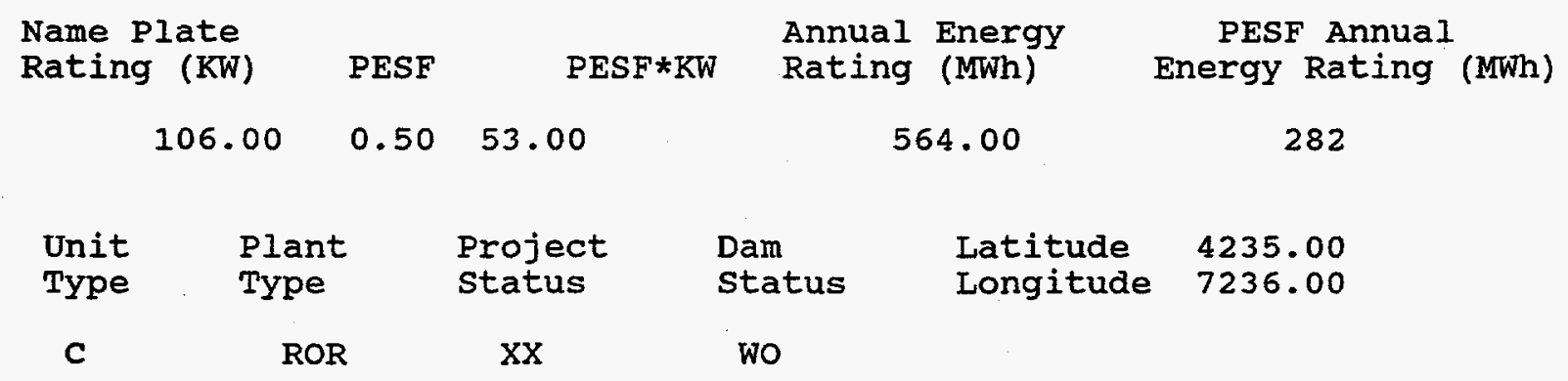

\section{Factor}

wild/Scenic Protection

wild/Scenic Tributary or Upstream/Downstream wild/Scenic Location Cultural value

Fish Presence Value

Geologic Value

Historic Value

other value

Recreation Value

Scenic Value

\section{Exist Prob}

Factor

0.90

Wildife Value

Threatened/Endangered Fish Threatened/Endangered Wildlife $Y$

Exist Prob

$\begin{array}{ll}0.75 & \text { Federal Land code } 103 \\ 0.90 & \text { Federal Land code } 104\end{array}$

0.90 Federal Land Code 105

Y 0.75 Federal Land code 105

Y 0.75 Federal Land code 107

$Y \quad 0.75$ Federal Land code 108

0.90 Federal Land code 198

0.90
MA 
FERC

Number

Plant Name

05666B GREEN ONE(MILI 1)

County Name

FRANKLIN stream

GREEN R, DEERFIELD R

River Basin

CONNECTICUT RIVER BASIN

Class Owner Name

M GREENFIELD, TOWN OF

$\begin{array}{lcccc}\begin{array}{l}\text { Name Plate } \\ \text { Rating (KW) }\end{array} & \text { PESF } & \text { PESF*KW } & \begin{array}{l}\text { Annual Energy } \\ \text { Rating (MWh) }\end{array} & \begin{array}{c}\text { PESF Annual } \\ \text { Energy Rating (MWh) }\end{array} \\ 71.00 & 0.50 & 35.50 & 336.00 & 168\end{array}$

$\begin{array}{lllll}\text { Unit } & \text { Plant } & \text { Project } & \text { Dam } & \text { Latitude } 4235.00 \\ \text { Type } & \text { Type } & \text { Status } & \text { Status } & \text { Longitude } 7236.00\end{array}$

C ROR XX WO

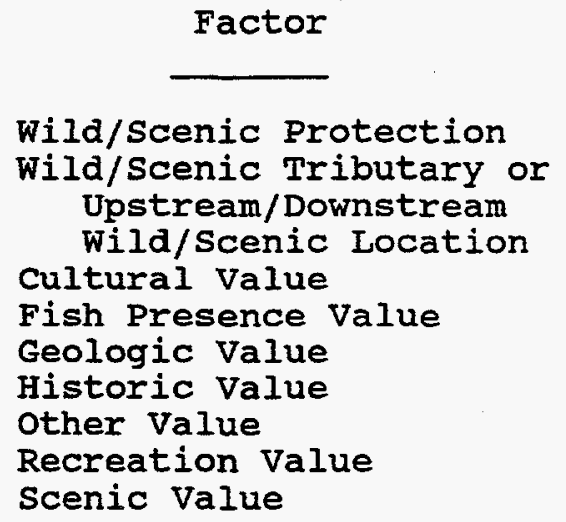

Exist Prob

0.90

Wildlife Value Threatened/Endangered Fish Threatened/Endangered Wildlife $Y$

Exist Prob

Factor

Y $\quad \begin{array}{ll}0.75 & \text { Federal Land code } 103 \\ 0.90 & \text { Federal Land code } 104\end{array}$

Federal Land code 105

$\mathrm{Y} \quad 0.75$

0.90

Federal Land Code 106

$\mathrm{Y} \quad 0.75$

Federal Land code 107

Federal Land Code 108

0.90

Federal Land Code 198

0.90
State

Name

MA 

R E S O U R C E
D A T A B A S E
L I S T I N G

DATE: $06 / 27 / 95$

PAGE NO: 13

FERC

Number

Plant Name

Stream

State

05826 SOUTH VILLAGE MILL

FRENCH R

Name

county Name

River Basin

WORCESTER

THAMES RIVER BASIN

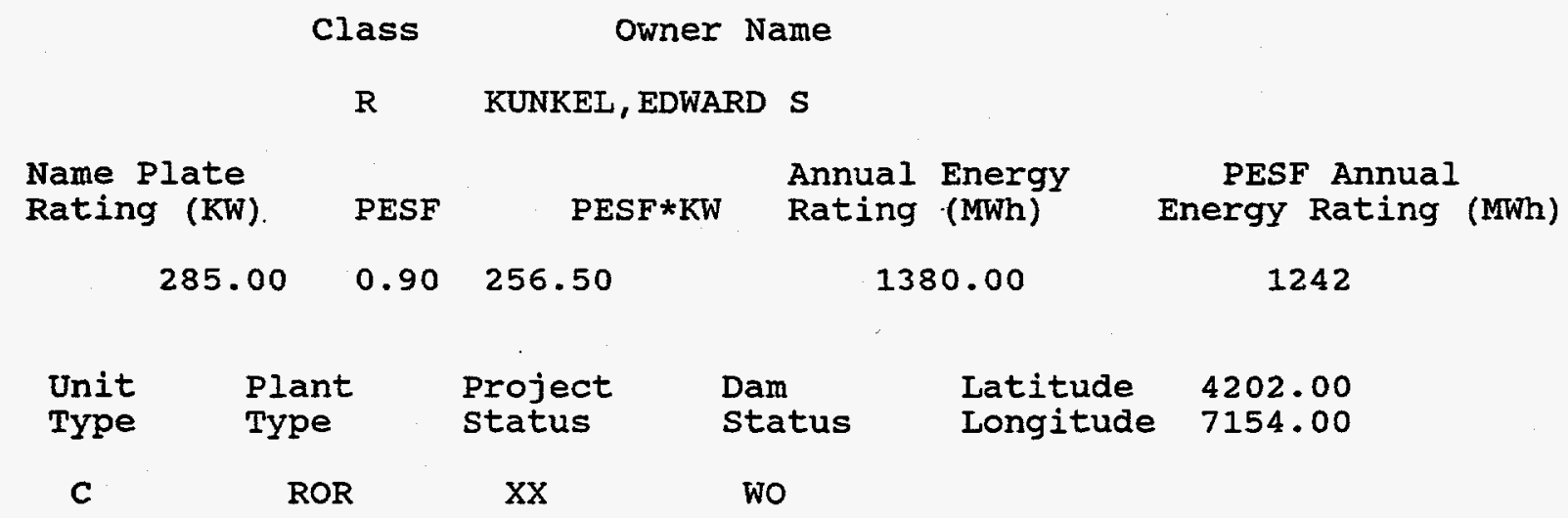

Factor

Exist Prob

Factor

Exist Prob

wild/Scenic Protection

wild/Scenic Tributary or

\subsection{Wildlife Value}

Threatened/Endangered Fish

Upstream/Downstream

wild/Scenic Location

cultural value

Fish Presence Value

Geologic Value

Historic Value

other Value

Recreation Value

Scenic Value
Threatened/Endangered wildlife

0.90 Federal Land Code 103

0.90 Federal Land code 104

0.90 Federal Land code 105

0.90 Federal Iand Code 106

0.90 Federal Land Code 107

0.90 Federal Land Code 108

0.90 Federal Land code 198

0.90
MA 
FERC Number Plant Name 05873 BARRE FALLS County Name WORCESTER class Owner Name $\mathbf{R}$ ENERGENICS SYSTEMS INC

Name Plate Rating (KW)

PESF

$$
\text { PESF*KW }
$$

Annual Energy

550.00

$$
0.50 \quad 275.00
$$

Plant Type

Type

$$
\text { STG }
$$

stream

WARE $R$, CHICOPEE $R$

River Basin

CONNECTICUT RIVER BASIN
State

Name

MA
PESF Annual Energy Rating (MWh)
Factor

Wild/Scenic Protection

Wild/Scenic Tributary or Upstream/Downstream wild/scenic Location Cultural Value

Fish Presence Value

Geologic Value

Historic Value

other value

Recreation Value

Scenic Value

$$
\begin{aligned}
& \text { Project } \\
& \text { status }
\end{aligned}
$$

$\mathrm{XX}$
Exist Prob

0.90

Wildlife Value Threatened/Endangered Fish Threatened/Endangered Wildlife $\begin{array}{lll}\text { Dam } & \text { Latitude } & 4221.00 \\ \text { Status } & \text { Longitude } & 7207.00\end{array}$

$\begin{array}{lll}\text { Dam } & \text { Latitude } & 4221.00 \\ \text { Status } & \text { Longitude } & 7207.00\end{array}$

$\mathrm{U}$
1350
0.50 Federal Land code 103

0.90 Federal Land code 104

0.90 Federal Land code 105

0.90 Federal Land code 106

0.90 Federal Land code 107

0.90 Federal Land Code 108

0.90 Federal Land Code 198
0.90
Exist Prob

0.90

0.90

0.90

0.90

0.90

0.90

0.90

0.90

0.90

0.90 
R E S O U C E D A T A B A S E I I S T I N G

DATE: $06 / 27 / 95$

PAGE NO: 15

FERC

Number

Plant Name

Stream

State

06096 NEW HOME SOUTH

MIILERS R

Name

county Name

River Basin

FRANKLIN

CONNECTICUT RIVER BASIN

$\begin{array}{cr}\text { Class } & \text { Owner Name } \\ \text { I } & \text { MINI WATT ELEC CO }\end{array}$

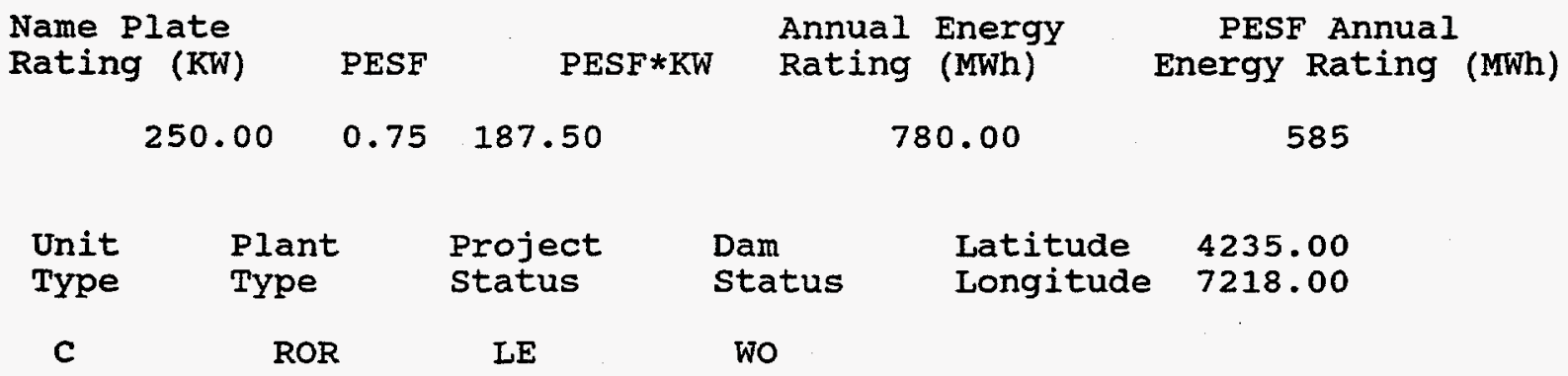

Factor

wild/scenic Protection

wild/Scenic Tributary or Upstream/Downstream wild/Scenic Location Cultural Value

Fish Presence Value

Geologic Value

Historic Value

other Value

Recreation Value

Scenic Value
Exist Prob

Factor

Exist Prob

0.90 Wildife Value

Threatened/Endangered Fish

Threatened/Endangered Wildlife

0.90 Federal Land code 103

Y 0.75 Federal Land code 104

0.90 Federal Lana code 105

0.90 Federal Land code 106

0.90 Federal Land code 107

0.90 Federal Land code 108

0.90 Federal Land Code 198

0.90
MA 
FERC

Number

Plant Name

06278

IAAKE RIPPLE DAM
County Name

WORCESTER

Class
Owner Name

M GRAFTON, TOWN OF

Name Plate Rating ( $\mathrm{KW}$ )

PESF

Annual Energy

PESF*KW

Rating (MWh)

26.00

$0.90 \quad 23.40$

122.00

Dam

status

Latitude Longitude

4212.00

status

$\mathrm{XX}$

WO

\begin{tabular}{|c|c|c|c|c|c|}
\hline $\begin{array}{l}\text { Unit } \\
\text { Type }\end{array}$ & $\begin{array}{l}\text { Plant } \\
\text { Type }\end{array}$ & $\begin{array}{l}\text { Project } \\
\text { status }\end{array}$ & $\begin{array}{l}\text { Dam } \\
\text { status }\end{array}$ & $\begin{array}{l}\text { Latitude } \\
\text { Longitude }\end{array}$ & $\begin{array}{l}4212.00 \\
7142.00\end{array}$ \\
\hline c & ROR & $\mathrm{XX}$ & wo & & \\
\hline
\end{tabular}

state

Name

MA
Factor

wild/scenic Protection

wild/Scenic Tributary or Upstream/Downstream wild/Scenic Location Cultural Value

Fish Presence Value

Geologic Value

Historic Value

Other value

Recreation value

Scenic Value
Exist Prob

0.90

wildlife Value

Threatened/Endangered Fish Threatened/Endangered Wildlife

0.90 Federal Land Code 103

0.90 Federal Land Code 104

0.90 Federal Land Code 105

0.90 Federal Land Code 106

0.90 Federal Land Code 107

0.90 Federal Land code 108

0.90 Federal Land code 198

0.90
PESF Annual Energy Rating (MWh)

109.80 


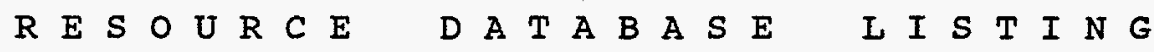

DATE: $06 / 27 / 95$

PAGE NO: 17

FERC

Number

06556

GLENALLAN MILLS

county Name

WORCESTER
Plant Name

\section{Class}

$\mathbf{R}$

Name Plate Rating ( $K W$ )

79.00
Stream

N BR MILLERS $\mathbf{R}$

River Basin

CONNECTICUT RIVER BASIN
State

Name

MA

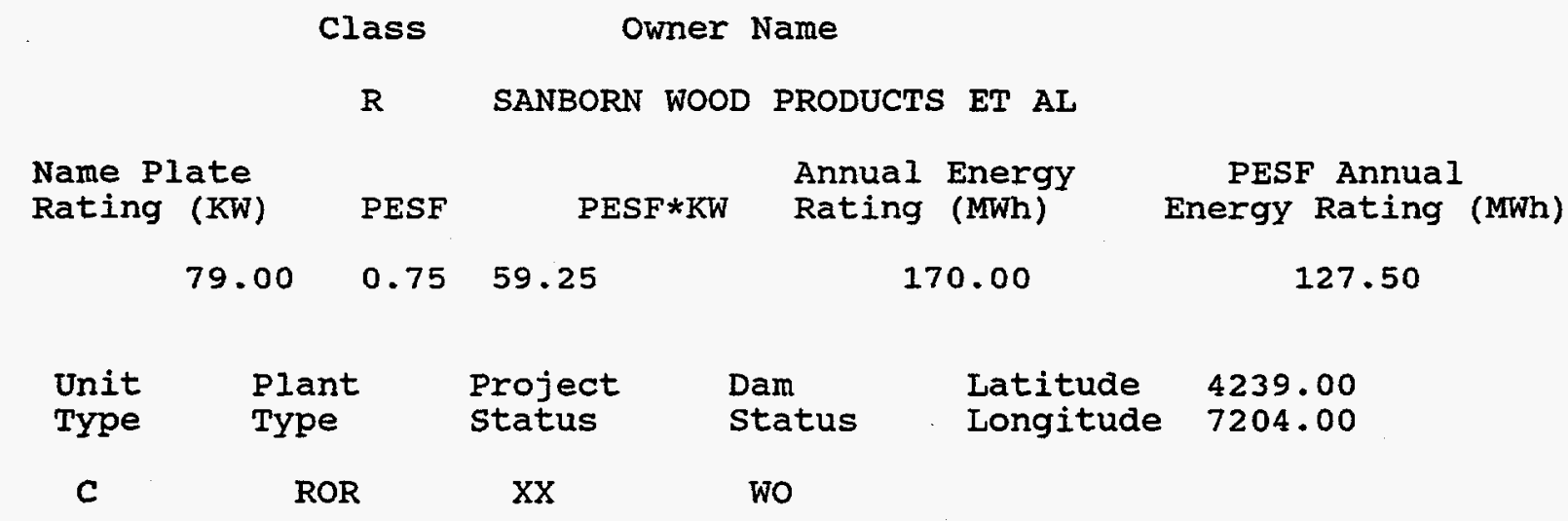

\section{Factor}

wild/Scenic Protection

wild/scenic Tributary or Upstream/Downstream wild/Scenic Location Cultural value Fish Presence Value Geologic Value Historic Value other Value Recreation Value Scenic Value
Exist Prob

0.90

Wildife Value Threatened/Endangered Fish Threatened/Endangered wildlife

0.90 Federal Land code 103

$\mathrm{Y}$

0.75

0.90

Federal Land code 104

Federal Land Code 105

0.90

Federal Land Code 106

0.90

0.90

Federal Land code 107

Federal Land code 108

0.90

0.90
Exist Prob

0.90

0.90

0.90

0.90

0.90

0.90

0.90

0.90

0.90

0.90 
R E S O U R C E

DATE: $06 / 27 / 95$
D A T A B A S E

L I $S$ T I N G

PAGE NO: 18
FERC

Number

Plant Name

06807 WALTER BAKER DAM

County Name

SUFFOIK
Stream

NEPONSET $R$

River Basin

MINOR RIVER BASINS
State

Name

MA

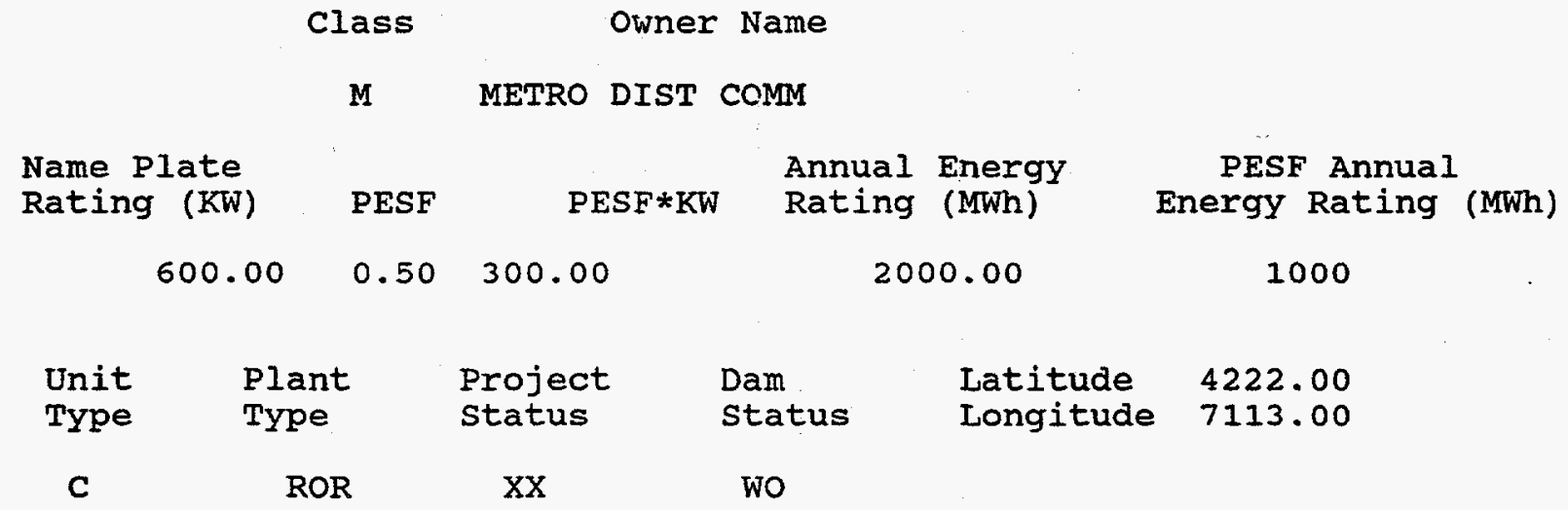

Factor

wild/Scenic Protection wild/Scenic Tributary or Upstream/Downstream wild/Scenic Location Cultural value

Fish Presence Value

Geologic Value Historic Value other value Recreation Value Scenic Value
Exist Prob

$\mathrm{Y}$

0.50

Wildife Value Threatened/Endangered Fish Threatened/Endangered Wildlife

0.90 Federal Land code 103

0.90 Federal. Land code 104

0.90 Federal Land Code 105

0.90 Federal Land code 106

0.90 Federal Land code 107

Y 0.75 Federal Land Code 108

0.90 Federal Land Code 198

0.90
Exist Prob

0.90

0.90

0.90

0.90

0.90

0.90

0.90

0.90

0.90

0.90 
RESOURCE DATA B A S E L I S T IN G

DATE: $06 / 27 / 95$

PAGE NO: 19

FERC

Number

Plant Name

Stream

State

Name

06966

BRASSWORKS

MILL $\mathbf{R}$

MA

County Name

HAMPSHIRE
River Basin

CONNECTICUT RIVER BASIN

\section{Class Owner Name}

R BRASSWORKS ASSOC

\begin{tabular}{|c|c|c|c|c|c|c|}
\hline \multicolumn{2}{|c|}{ Name Plate } & PESF & PESF *KW & $\begin{array}{l}\text { Anni } \\
\text { Rati }\end{array}$ & $\begin{array}{l}\text { Energy } \\
\text { (MWh) }\end{array}$ & $\begin{array}{l}\text { PESF Annual } \\
\text { Energy Rating (MWh) }\end{array}$ \\
\hline & 75.00 & 0.75 & 56.25 & & 0.00 & 262.50 \\
\hline $\begin{array}{l}\text { Unit } \\
\text { Type }\end{array}$ & $\begin{array}{l}\text { Pl } \\
\text { Ty }\end{array}$ & & $\begin{array}{l}\text { Project } \\
\text { status }\end{array}$ & $\begin{array}{l}\text { Dam } \\
\text { Status }\end{array}$ & $\begin{array}{l}\text { Latitude } \\
\text { Longitude }\end{array}$ & $\begin{array}{l}4224.00 \\
7245.00\end{array}$ \\
\hline C & & & $\mathrm{XX}$ & wo & & \\
\hline
\end{tabular}

Factor

Exist Prob

Factor

Exist Prob

Wild/Scenic Protection

wild/Scenic Tributary or Upstream/Downstream wild/Scenic Location Cultural value Fish Presence Value Geologic Value Historic Value other Value Recreation Value scenic Value

\subsection{Wildlife Value} Threatened/Endangered Fish Threatened/Endangered wildlife

0.90

0.90

0.90

$Y$

0.75

0.90

0.90

Federal Land code 104

Federal Land Code 105

0.90

Federal Land Code 106

0.90 Federal Land Code 107

0.90 Federal Land Code 108

0.90 Federal Land Code 198

0.90
0.90

0.90

0.90

0.90

0.90

0.90

0.90 

R E S O U R C E
D A T A B A S E
L I S T I N G

DATE: $06 / 27 / 95$

PAGE NO: 20

FERC

Number

Plant Name

Stream

state

Name

07367 WATERSHOPS

MILL R

MA

County Name

River Basin

HAMPDEN

CONNECTICUT RIVER BASIN

$\begin{array}{cc}\text { Class } & \text { Owner Name } \\ \text { R } & \text { HYDRO DEV OF NEW ENGLAND INC }\end{array}$

$\begin{array}{rrrrr}\begin{array}{l}\text { Name Plate } \\ \text { Rating (KW) }\end{array} & \text { PESF } & \text { PESF*KW } & \begin{array}{l}\text { Annual Energy } \\ \text { Rating (MWh) }\end{array} & \begin{array}{c}\text { PESF Annual } \\ \text { Energy Rating (MWh) }\end{array} \\ 100.00 & 0.75 & 75.00 & 500.00 & 375\end{array}$

$\begin{array}{lllll}\text { Unit } & \text { Plant } & \text { Project } & \text { Dam } & \text { Latitude } 4205.00 \\ \text { Type } & \text { Type } & \text { Status } & \text { Status } & \text { Longitude } 7228.00\end{array}$

C ROR $\mathrm{XX}$ Wo

Factor

wild/Scenic Protection

Wild/Scenic Tributary or Upstream/Downstream wild/Scenic Location Cultural value

Fish Presence Value

Geologic Value

Historic Value

other Value

Recreation value

Scenic Value
Exist Prob

Factor

Exist Prob

0.90

Wildlife Value

Threatened/Endangered Fish

Threatened/Endangered Wildlife

0.90

0.90

0.90

$\mathrm{Y} \quad 0.75$ Federal Land code 103

0.90 Federal Land Code 104

0.90

0.90

0.90 Federal Land Code 105

0.90 Federal Land code 106

0.90 Federal Land code 107

0.90 Federal Land code 108

0.90 Federal Land code 198

0.90
0.90

0.90

0.90

0.90

0.90 

R E S O U R E
D A T A B A S E
L I S T $N$ G

DATE: $06 / 27 / 95$

PAGE NO: 21

FERC

Number

Plant Name

Stream

State

07486

CORDINGLY DAM

CHARLES $R$

Name

\section{6}

county Name

River Basin

MIDDLESEX

MINOR RIVER BASINS

MA

$\begin{array}{cr}\text { class } & \text { Owner Name } \\ \text { M } & \text { METRO DIST COMM }\end{array}$

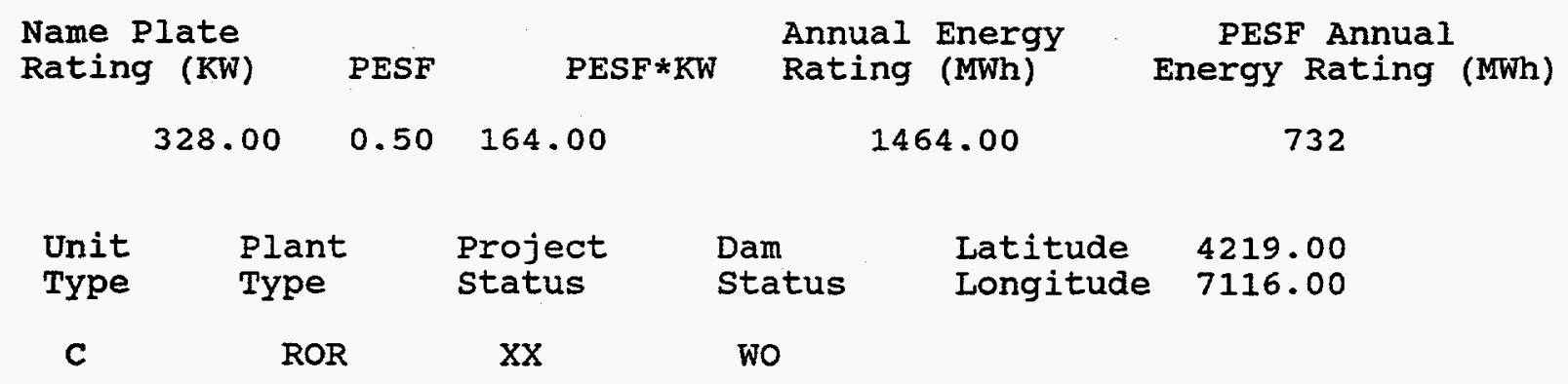

Factor

wild/Scenic Protection

wild/Scenic Tributary or Upstream/Downstream wild/Scenic Location Cultural value

Fish Presence Value

Geologic Value

Historic Value

other Value

Recreation Value

Scenic Value
Exist Prob

Factor

Exist Prob

0.90 Wildlife Value

Threatened/Endangered Fish

Threatened/Endangered Wildife

0.90 Federal Land code 103

0.90 Federal Land code 104

0.90 Federal Land Code 105

0.90 Federal Land Code 106

0.90 Federal Land Code 107

$Y \quad 0.75$ Federal Land code 108

Y 0.75 Federal Land Code 198

0.90
0.90

0.90

0.90

0.90

0.90

0.90

0.90

0.90

0.90

0.90 
FERC

Number

Plant Name

07917 WILLOW MILL

County Name

BERKSHIRE

$\begin{array}{lc}\text { Stream } & \begin{array}{c}\text { State } \\ \text { Name }\end{array} \\ \text { HOUSATONIC R } & \text { MA } \\ \text { River Basin } & \end{array}$

HOUSATONIC RIVER BASIN

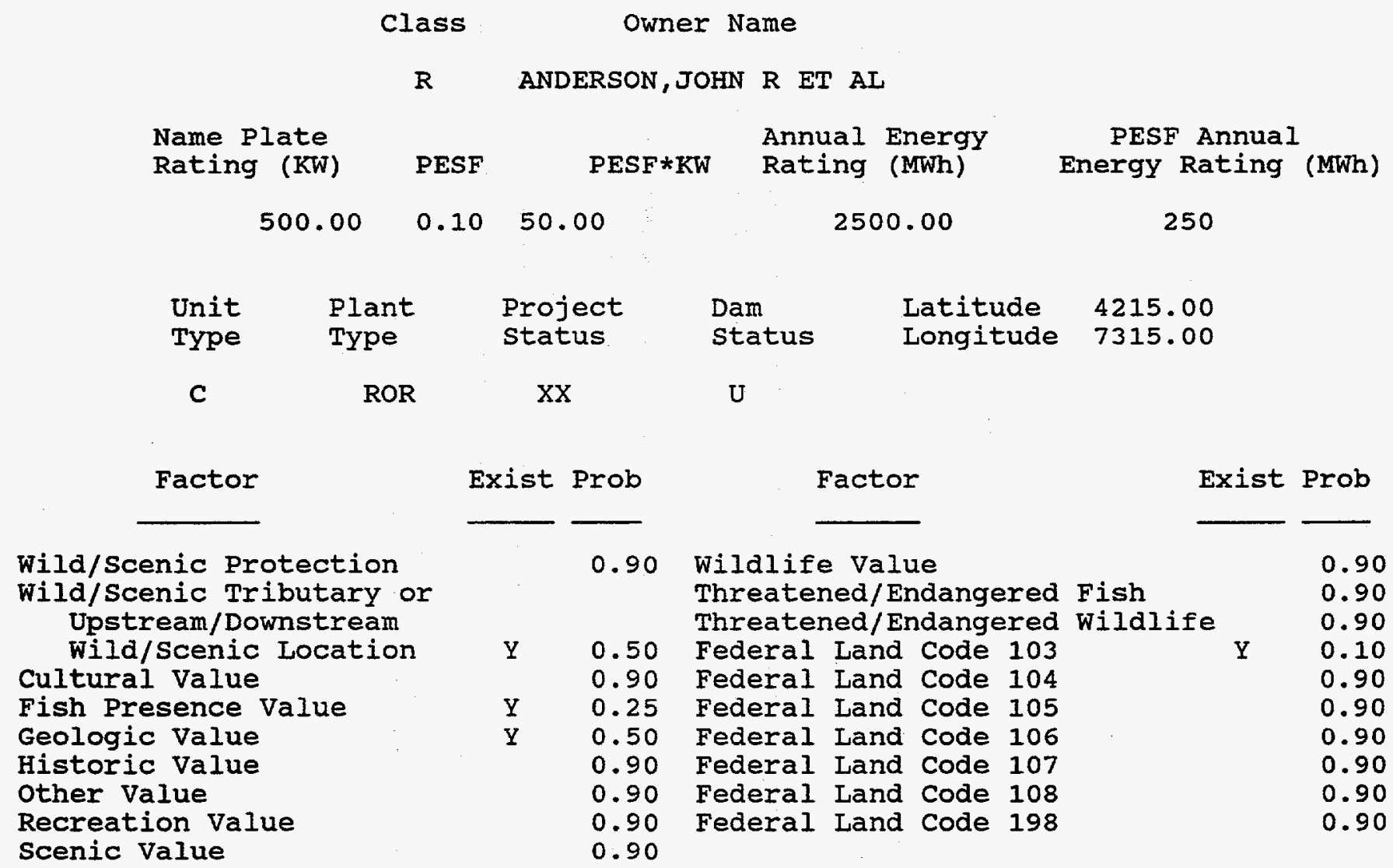


DATE: $06 / 27 / 95$

\section{FERC}

Number

Plant Name

08012 HUNTS POND

county Name

WORCESTER
PAGE NO: 23

Stream

State

Name

MILLERS $\mathbf{R}$

MA

River Basin

CONNECTICUT RIVER BASIN

Class Owner Name

I BEHRENS ENERGY SYSTEMS INC

Name Plate

Rating (KW)

200.00

PESF

Annual Energy

PESF*KW

Rating

(MWh)

374.00

$0.50 \quad 100.00$
PESF Annua1 Energy Rating (MWh)

187

\begin{tabular}{|c|c|c|c|c|c|}
\hline $\begin{array}{l}\text { Unit } \\
\text { Type }\end{array}$ & $\begin{array}{l}\text { Plant } \\
\text { Type }\end{array}$ & $\begin{array}{l}\text { Project } \\
\text { status }\end{array}$ & $\begin{array}{l}\text { Dam } \\
\text { Status }\end{array}$ & $\begin{array}{l}\text { Latitude } \\
\text { Longitude }\end{array}$ & $\begin{array}{l}4240.00 \\
7202.00\end{array}$ \\
\hline & ROR & LE & $W$ & & \\
\hline
\end{tabular}

Factor

Wild/scenic Protection

wild/Scenic Tributary or

Upstream/Downstream

wild/Scenic Location

Cultural Value

Fish Presence Value

Geologic Value

Historic Value

other Value

Recreation Value

Scenic Value
Exist Prob

Factor

Exist Prob

\begin{tabular}{|c|c|}
\hline & 0.90 \\
\hline$Y$ & 0.75 \\
\hline$Y$ & 0.75 \\
\hline & 0.90 \\
\hline & 0.90 \\
\hline $\mathrm{Y}$ & 0.75 \\
\hline & 0.90 \\
\hline & $\begin{array}{l}0.90 \\
0.90\end{array}$ \\
\hline
\end{tabular}

Wildlife Value

Threatened/Endangered Fish

Threatened/Endangered Wildlife

Federal Land code 103

Federal Land Code 104

Federal Land code 105

Federal Land code 106

Federal Land Code 107

Federal Land Code $108^{\circ}$

Federal Land Code 198
0.90

0.90

0.90

0.90

0.90

0.90

0.90

0.90

0.90

0.90 
RESOU R C E D A T A B A S E L I S T I N G

DATE: $06 / 27 / 95$

PAGE NO: 24

FERC

Number

Plant Name

Stream

state

Name

08234A MILLERS ONE

MILLERS $R$

MA

County Name

FRANKLIN

class

Owner Name

R DUPONT COMPANY INC

Name Plate

Rating (KW)

PESF

PESF*KW

Annual Energy

1090.00

$0.50 \quad 545.00$

Rating (MWh)

5310.00

Dam Latitude

Status

Longitude

4234.00

status

XX

WO
Factor

wild/Scenic Protection

Wild/Scenic Tributary or

Upstream/Downstream

wild/Scenic Location

cultural value

Fish Presence Value

Geologic Value

Historic Value

other Value

Recreation value

Scenic value
Exist Prob

0.90

wildlife Value

Threatened/Endangered Fish

Threatened/Endangered Wildlife

Y 0.75 Federal Land Code 103

$Y \quad 0.75$ Federal Land code 104

0.90 Federal Land Code 105

0.90 Federal Land code 106

0.90 Federal Land Code 107

0.90 Federal Land Code 108

0.90 Federal Land code 198

0.90
PESF Annual Energy Rating (MWh)

2655 
RES O U R E D A T A B A S E I I S T I N G

DATE: $06 / 27 / 95$

PAGE NO: 25

FERC

Number Plant Name

stream

state

08234B MILLERS TWO

MILLERS R

Name

County Name

FRANKLIN

River Basin

CONNECTICUT RIVER BASIN

Class Owner Name

$\mathrm{R}$ DUPONT COMPANY INC

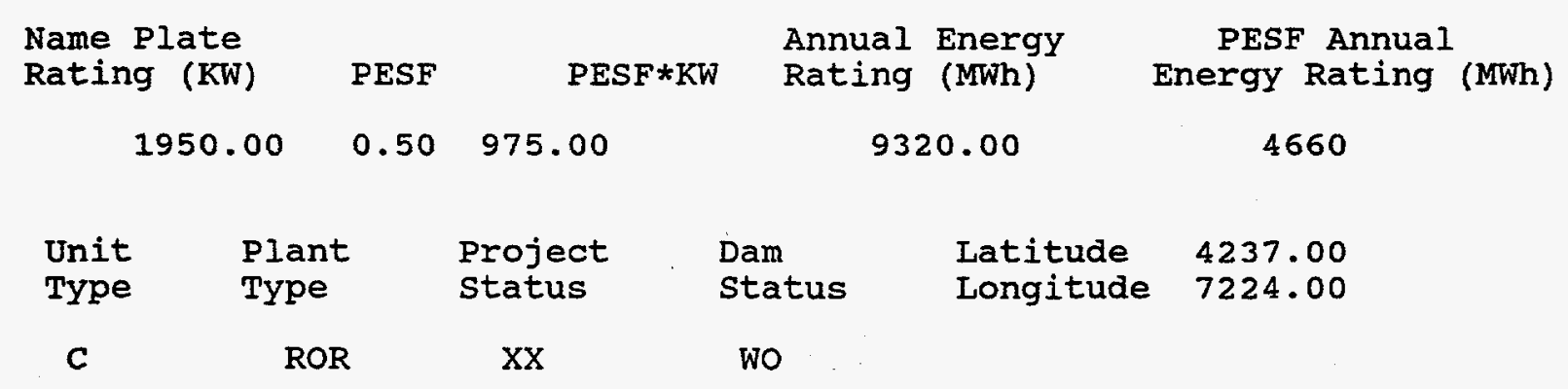

Factor

wild/scenic Protection

wild/Scenic Tributary or Upstream/Downstream wild/Scenic Location Cultural value Fish Presence Value Geologic Value Historic Value other Value Recreation Value Scenic Value
Exist Prob

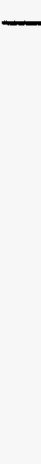

Factor

Exist Prob

\subsection{Wildife Value Threatened/Endangered Fish} Threatened/Endangered Wildlife

$\mathrm{Y} \quad 0.75$ Federal Land Code 103

$\mathrm{Y} \quad 0.75$ Federal Land Code 104

0.90 Federal Land Code 105

0.90 Federal Land code 106

0.90 Federal Land code 107

0.90 Federal Land code 108

0.90 Federal Land Code 198

0.90
MA 
DATE: $06 / 27 / 95$

PAGE NO: 26

FERC

Number

Plant Name

08234C MILLERS THREE

county Name

FRANKLIN
MILLERS $\mathrm{R}$

stream

State

Name

MA

River Basin

CONNECTICUT RIVER BASIN

Class Owner Name

$\mathrm{R}$ DUPONT COMPANY INC

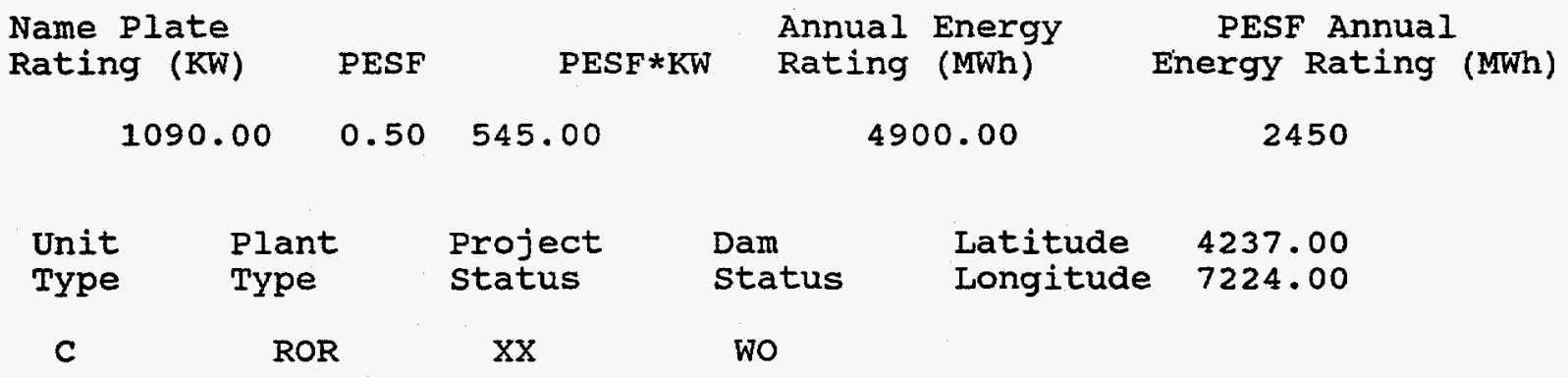

Factor

Wild/scenic Protection wild/Scenic Tributary or Upstream/Downstream wild/Scenic Location Cultural value Fish Presence Value Geologic Value Historic Value other Value Recreation Value Scenic Value
Exist Prob

0.90

0.75

0.75

0.90

0.90

0.90

0.90

0.90

0.90
Factor

Exist Prob

Wilalife Value Threatened/Endangered Fish Threatened/Endangered Wildlife

0.90

0.90

0.90

Federal Land code 103

0.90

Federal Land code 104

0.90

Federal Land Code 105

Federal Land Code 106

0.90

0.90

Federal Land Code 107

0.90

0.90

Federal Land Code 108

Federal Land code 198

0.90 

R E S O U R C E
D A $A$ A B A $\quad$ S E
L I S T I N G

DATE: $06 / 27 / 95$

PAGE NO: 27

FERC

Number

Plant Name

Stream

state

08234D MILLERS FOUR

MILLERS $\mathrm{R}$

Name

county Name

River Basin

FRANKLIN

CONNECTICUT RIVER BASIN

\section{Class Owner Name}

R DUPONT COMPANY INC

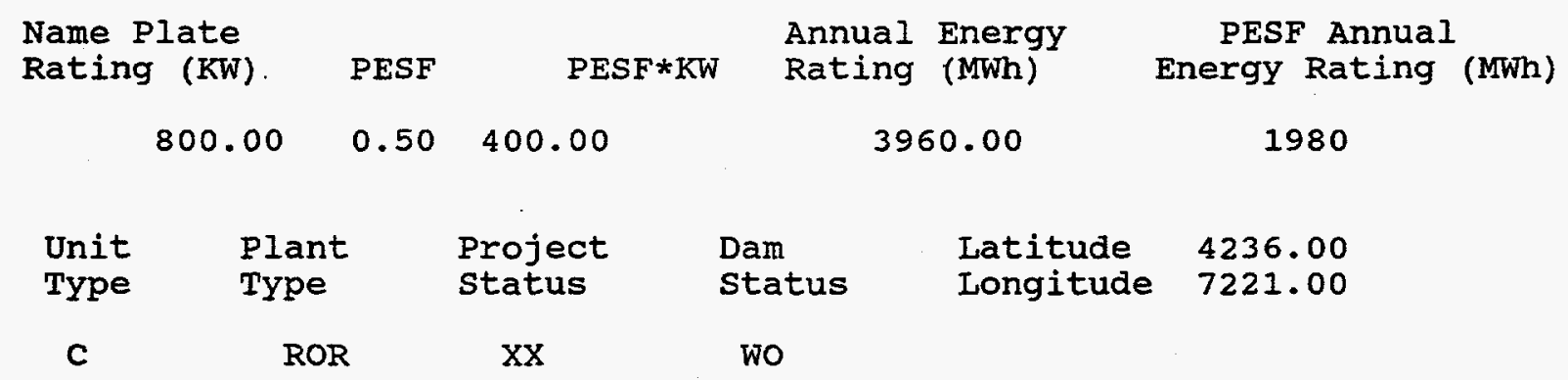

Factor

Wild/Scenic Protection

wild/Scenic Tributary or Upstream/Downstream wild/Scenic Location Cultural value

Fish Presence Value

Geologic Value

Historic Value

other Value

Recreation value

Scenic Value
Exist Prob

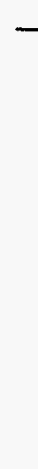

\section{Factor}

Exist Prob

$\begin{array}{ll}0.90 & \text { Wildife Value } \\ & \text { Threatened/Endangered Fish } \\ & \text { Threatened/Endangered wildlife } \\ 0.75 & \text { Federal Land Code } 103 \\ 0.75 & \text { Federal Land Code } 104 \\ 0.90 & \text { Federal Land Code } 105 \\ 0.90 & \text { Federal Land Code } 106 \\ 0.90 & \text { Federal Land Code } 107 \\ 0.90 & \text { Federal Land Code } 108 \\ 0.90 & \text { Federal Land Code } 198 \\ 0.90 & \end{array}$

MA 
FERC

Number

Plant Name

08234E MILLERS FIVE

county Name

FRANKLIN state

Name

MA

MILLERS R

stream

River Basin

CONNECTICUT RIVER BASIN

Class Owner Name

R DUPONT COMPANY INC

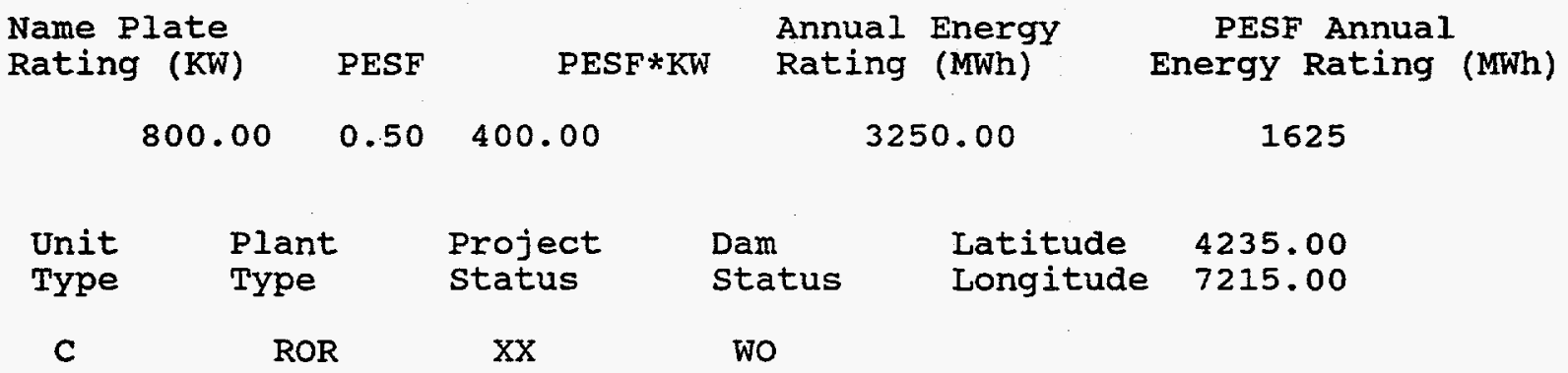

Factor

Exist Prob

Factor

Exist Prob

wild/Scenic Protection

wild/Scenic Tributary or

Upstream/ Downstream

wild/scenic Location

Cultural value

Fish Presence Value

Geologic value

Historic Value

other value

Recreation Value

-

0.90

Wildlife Value

0.90

Threatened/Endangered Fish

0.90

Threatened/Endangered Wildiife

0.90

Y 0.75 Federal Land Code 103

0.90

Y 0.75 Federal Land code 104

0.90

0.90

Federal Land code 105

0.90

0.90 Federal Land Code 106

0.90

0.90 Federal Land Code 107

0.90

0.90

Federal Land code 108

0.90

0.90

Federal Land Code 198

0.90

Scenic Value

0.90 
RESOURCE D A T A B A S E I I S T ING

DATE: $06 / 27 / 95$

PAGE NO: 29

FERC

Number

08293

KENNEY POND

County Name

WORCESTER

\section{Class}

R KENNEY POND HYDRO CO

Name Plate

Rating (KW)

PESF

100.00

$$
0.50 \quad 50.00
$$

Unit
Type

Plant
Type

C

ROR

Exist Prob

Factor

wild/Scenic Protection

wild/Scenic Tributary or

Upstream/Downstream

wild/Scenic Location

Cultural Value

Fish Presence Value

Geologic Value

Historic Value

other Value

Recreation Value

Scenic Value
Annual Energy

PESF * KW

Rating (MWh)

319.00

Latitude

Longitude

4237.00

Project status

$\mathrm{XX}$

$\begin{array}{ll}\text { Dam } & \text { Latitude } \\ \text { Status } & \begin{array}{l}\text { Longitude } \\ \text { wo }\end{array}\end{array}$

Factor

Exist Prob

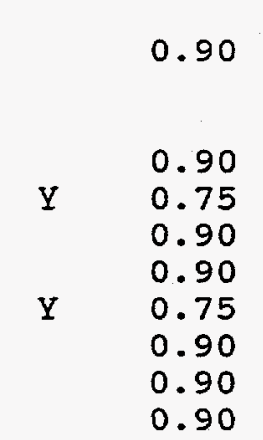

Wildife Value

Threatened/Endangered Fish

State

Name

MA

PESF Annual Energy Rating (MWh)

$$
159.50
$$

0.90

0.90

0.90

0.90

0.90

0.90

0.90

0.90

0.90

0.90 
FERC Number Plant Name 08350 LITTLEVILLE

County Name HAMPSHIRE
State Name

M BR WESTFIELD $R$

MA

$$
\text { River Basin }
$$

CONNECTICUT RIVER BASIN

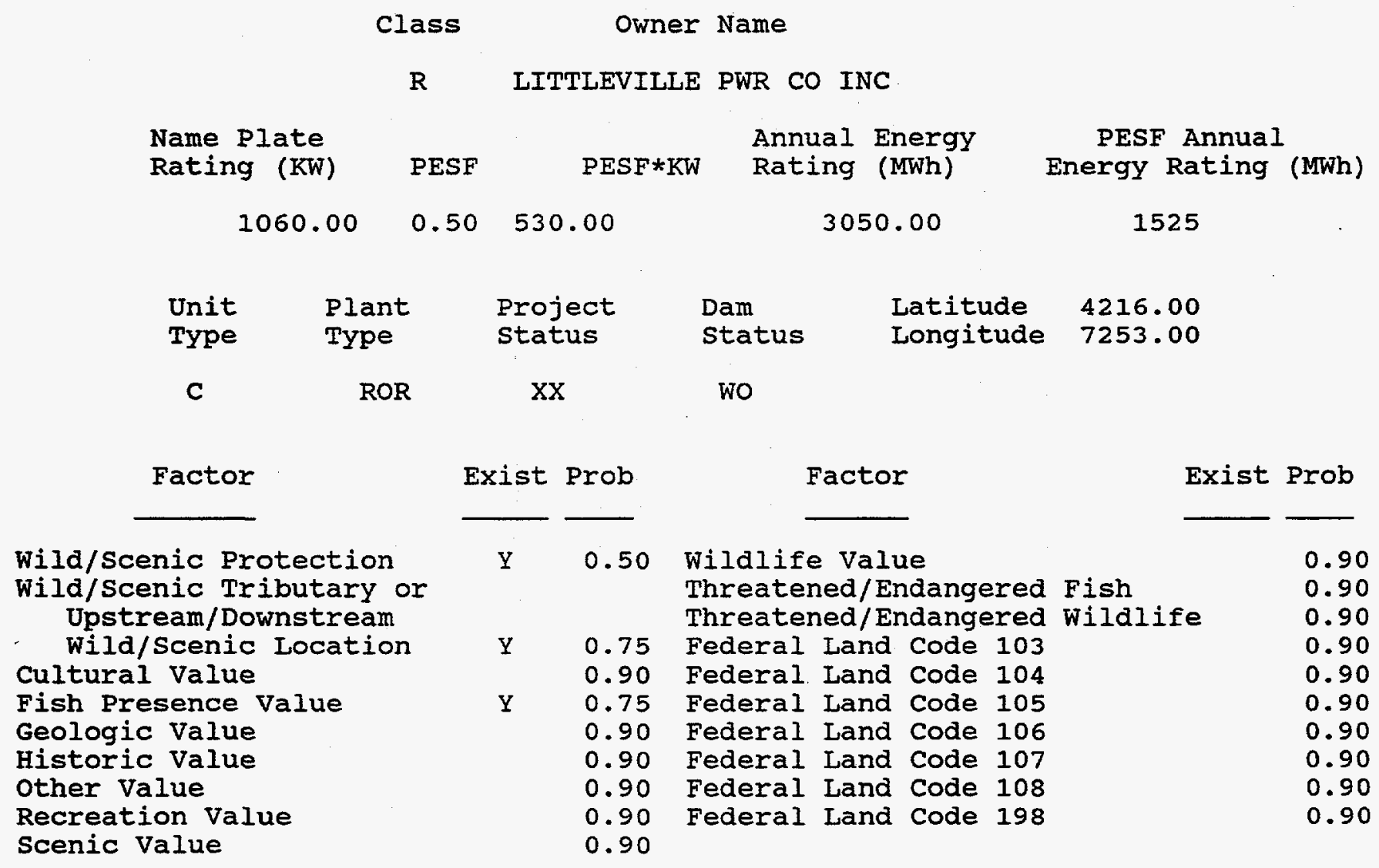


R E S O U R C E D A T A B A S E L I S T I N G

DATE: $06 / 27 / 95$

PAGE NO: 31

FERC

Number

Plant Name

08424 BILLERICA

County Name

MIDDLESEX stream

CONCORD R

River Basin

MERRIMACK RIVER BASIN
State

Name

MA

Class Owner Name

$R$ ANDERSON, JOHN R ET AL

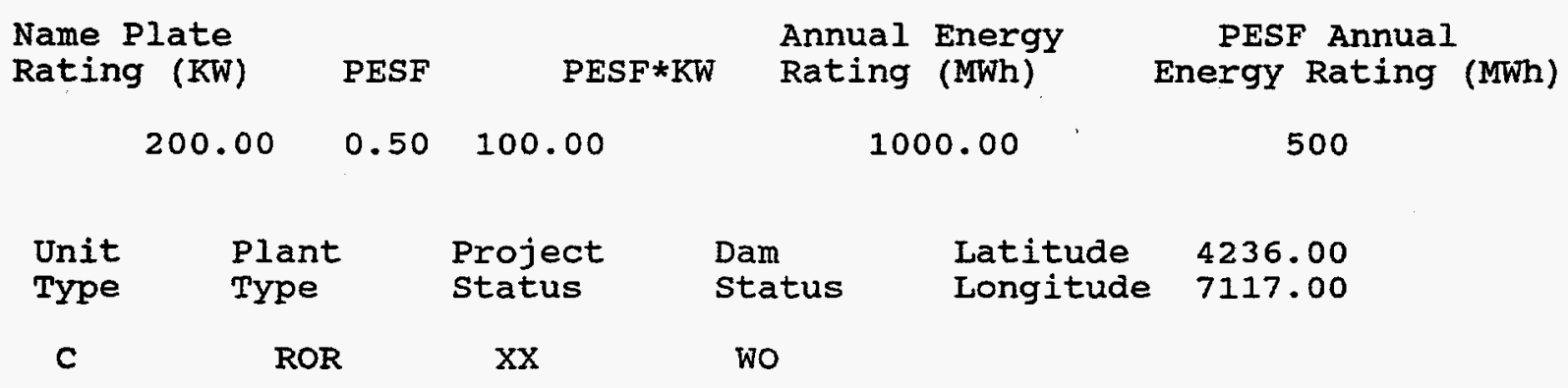

Factor

Wild/Scenic Protection

Wild/Scenic Tributary or

Upstream/Downstream wild/Scenic Location Cultural value Fish Presence Value Geologic Value Historic Value other Value Recreation Value Scenic Value
Exist Prob

Y

$$
0.50
$$

Wildife Value Threatened/Endangered Fish Threatened/Endangered Wildlife

Exist Prob

Y 0.75 Federal Land code 103

0.90 Federal Land code 104

0.90 Federal Land Code 105

0.90 Federal Land code 106

0.90 Federal Land Code 107

0.90 Federal Land code 108

$Y \quad 0.75$ Federal Land Code 198
0.90

0.90

0.90

0.90

0.90

0.90

0.90

0.90

0.90

0.90 


\section{FERC}

Number

08449
Plant Name

BERNARDSTON GRAIN MILL

county Name

FRANKLIN
River Basin

CONNECTICUT RIVER BASIN $\begin{array}{cc}\text { Class } & \text { Owner Name } \\ R & \text { ATWILL, STEVEN \& SUSAN }\end{array}$

Name Plate

Rating ( $\mathrm{KW}$ )

PESF

$\mathrm{PESF} * \mathrm{KW}$

Annual Energy

Rating (MWh)

$80.00 \quad 0.90 \quad 72.00$

300.00

Latitude

Longitude

4240.00

status

Dam

Status

7232.00 c

ROR

$\mathrm{xx}$

wo

state

Name

MA

\author{
Factor \\ Wild/Scenic Protection \\ wild/scenic Tributary or \\ Upstream/Downstream \\ wild/Scenic Location \\ Cultural Value \\ Fish Presence Value \\ Geologic Value \\ Historic Value \\ Other Value \\ Recreation value \\ Scenic Value
}

Exist Prob

0.90

Wildife Value

Threatened/Endangered Fish Threatened/Endangered Wildlife

0.90 Federal Land Code 103

0.90 Federal Land Code 104

0.90 Federal Land Code 105

0.90 Federal Land Code 106

0.90 Federal Land Code 107

0.90 Federal Land Code 108

0.90 Federal Land Code 198

0.90
PESF Annual Energy Rating (MWh)

270 
RESOURCE DA TA B A S E I I S T I N G

DATE: $06 / 27 / 95$

PAGE NO: 33

FERC

Number

08641

BALLARDVALE

County Name

ESSEX

Class

R BURIINGTON ENERGY DEV ASSOC

Name Plate

Rating (KW)

40.00
Annual Energy Rating (MWh)

200.00
River Basin

MERRIMACK RIVER BASIN state

Name

MA

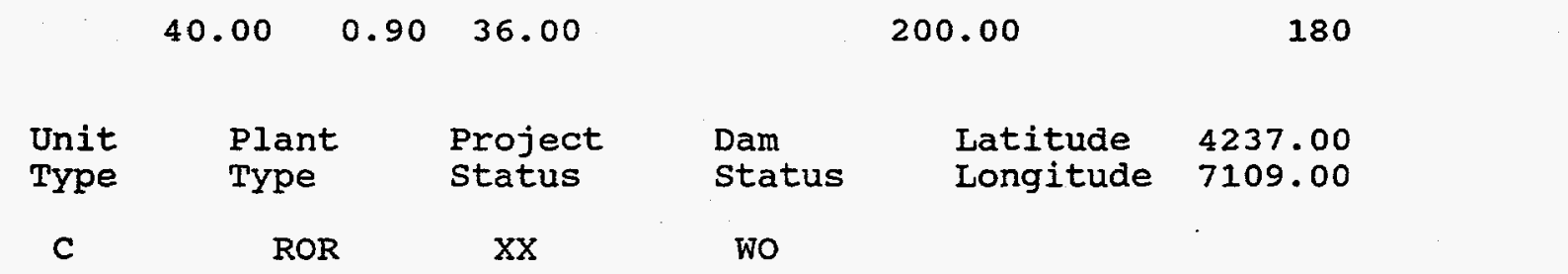

Factor

wild/scenic Protection

wild/scenic Tributary or

Upstream/Downstream

wild/Scenic Location

Cultural value

Fish Presence Value

Geologic Value

Historic Value

other Value

Recreation Value

Scenic Value
Exist Prob

0.90

Wildlife Value

Threatened/Endangered Fish

Threatened/Endangered Wildlife

0.90 Federal Land Code 103

0.90 Federal Land Code 104

0.90 Federal Land Code 105

0.90 Federal Land Code 106

0.90 Federal Land code 107

0.90 Federal Land code 108

0.90 Federal Land Code 198

0.90
PESF Annual Energy Rating (MWh)

180
0.90

0.90

0.90

0.90

0.90

0.90

0.90

0.90

0.90

0.90 
DATE: $06 / 27 / 95$

PAGE NO: 34

FERC

Number

08652
Plant Name

PLEASANT STREET

County Name

MIDDLESEX stream

BEAVER BK, MERRIMACK R

River Basin

MERRIMACK RIVER BASIN
State

Name

MA

Class Owner Name

R BURLINGTON ENERGY DEV ASSOC

$\begin{array}{rrrcr}\begin{array}{l}\text { Name Plate } \\ \text { Rating (KW) }\end{array} & \text { PESF } & \text { PESF*KW } & \begin{array}{l}\text { Annual Energy } \\ \text { Rating (MWh) }\end{array} & \begin{array}{c}\text { PESF Annual } \\ \text { Energy Rating (MWh) }\end{array} \\ 65.00 & 0.75 & 48.75 & 325.00 & 243.75\end{array}$

\begin{tabular}{|c|c|c|c|c|}
\hline $\begin{array}{l}\text { Unit } \\
\text { Type }\end{array}$ & $\begin{array}{l}\text { Plant } \\
\text { Type }\end{array}$ & $\begin{array}{l}\text { Project } \\
\text { status }\end{array}$ & $\begin{array}{l}\text { Dam } \\
\text { status }\end{array}$ & $\begin{array}{l}\text { Latitude } \\
\text { Longitude }\end{array}$ \\
\hline
\end{tabular}

C

ROR

$\mathrm{XX}$

Wo

Factor

Exist Prob

Factor

Exist Prob

wild/Scenic Protection

wild/Scenic Tributary or Upstream/Downstream

wild/Scenic Location

Cultural value

Fish Presence Value

Geologic Value

Historic Value

other Value

Recreation Value

scenic Value

Exist

0.90

Wildlife Value

Threatened/Endangered Fish

Threatened/Endangered wildlife

$Y \quad 0.75$ Federal Land Code 103

0.90 Federal Land code 104

0.90 Federal Land Code 105

0.90 Federal Land Code 106

0.90 Federal Land Code 107

0.90 Federal Land code 108

0.90 Federal Land code 198

0.90
0.90

0.90

0.90

0.90

0.90

0.90

0.90

0.90

0.90

0.90 
R E S O UR C E D A T A B A S E L I S T I N G

DATE: $06 / 27 / 95$

PAGE NO: 35

FERC

Number

Plant Name

08653 ANDOVER

County Name

ESSEX

\section{Class}

$\mathbf{R}$

BURLINGTON ENERGY DEV ASSOC

Stream

SHAWSHEEN $R$

River Basin

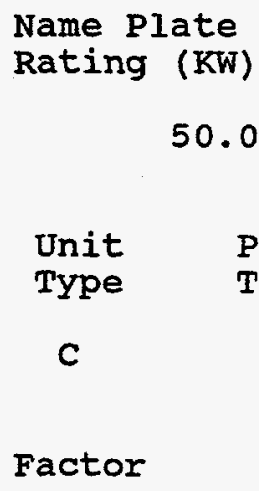

\section{Factor}

MERRIMACK RIVER BASIN

$$
50.00 \quad 0.90 \quad 45.00
$$$$
250.00
$$

$\begin{array}{ccclll}\text { Unit } & \text { Plant } & \text { Project } & \text { Dam } & \text { Latitude } & 4239.00 \\ \text { Type } & \text { Type } & \text { Status } & \text { Status } & \text { Longitude } 7108.00 \\ \text { C } & \text { ROR } & \text { XX } & \text { WO } & \end{array}$

state

Name

MA

PESF Annual Energy Rating (MWh)

$$
225
$$

wild/Scenic Protection wild/Scenic Tributary or Upstream/Downstream wild/Scenic Location cultural value Fish Presence value Geologic Value Historic Value other Value Recreation Value Scenic Value
Exist Prob

$$
0.90
$$

Wildlife Value

0.90

0.90

0.90

0.90

0.90

0.90

0.90

0.90 Threatened/Endangered Fish Threatened/Endangered Wildlife

Factor

Exist Prob

Federal Land Code 103

Federal Land Code 104

Federal Land Code 105

Federal Land Code 106

Federal Land code 107

Federal Land code 108

Federal Land code 198
0.90

0.90

0.90

0.90

0.90

0.90

0.90

0.90

0.90

0.90 
DATE: $06 / 27 / 95$

PAGE NO: 36

FERC

Number

Plant Name

08890

NEWTON UPPER FALLS

County Name

MIDDLESEX

\section{Class}

Owner Name

$\mathbf{R}$

\section{BURIINGTON}

ENERGY

Annual Energy

Name Plate Rating (KW)

350.00

0.50

175.00

Plant Type

ROR

$$
\begin{aligned}
& \text { Project } \\
& \text { Status }
\end{aligned}
$$

$\mathrm{XX}$

$$
1750.00
$$

state

Name

MA
PESF Annual Energy Rating (MWh)

875

$\begin{array}{lllll}\text { Unit } & \text { Plant } & \text { Project } & \text { Dam } & \text { Latitude } 4219.00 \\ \text { Type } & \text { Type } & \text { Status } & \text { Status } & \text { Longitude } 7114.00\end{array}$

Factor

Exist Prob

Factor

Exist Prob

wild/Scenic Protection wild/Scenic Tributary or

Upstream/ Downstream wild/scenic Location Cultural value Fish Presence Value Geologic Value Historic Value other value Recreation Value Scenic Value
0.90 Wildlife Value Threatened/Endangered Fish Threatened/Endangered Wildiife

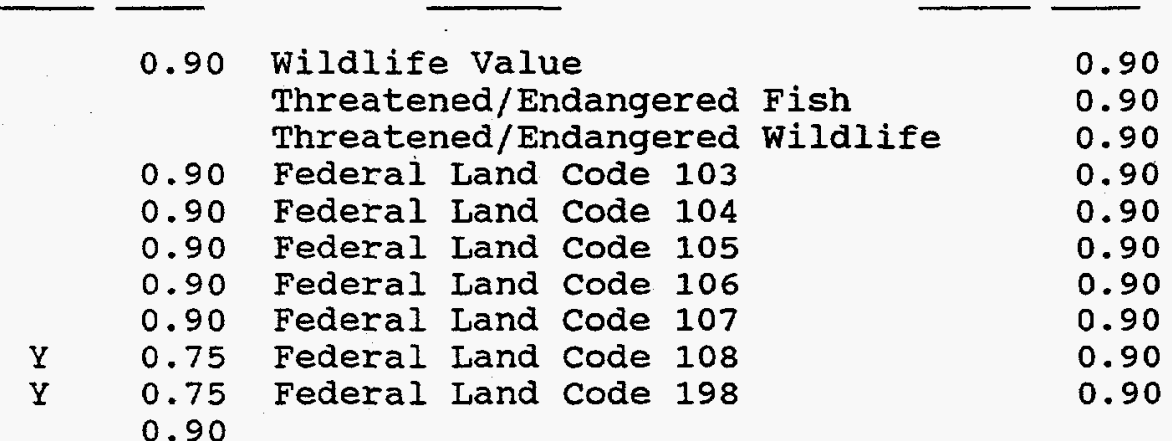


RESOURCE D A T A B A S E I I S T I N G

DATE: $06 / 27 / 95$

PAGE NO: 37

FERC

Number

08933
Plant Name

BEAR ROCK FALLS

county Name

BERKSHIRE
Stream

BEAR ROCK STR, HOUSATONIC R

River Basin

HOUSATONIC RIVER BASIN state

Name

MA

Class Owner Name

R BURLINGTON ENERGY DEV ASSOC

Name Plate

Rating (KW)

PESF

Annual Energy

Rating (MWh)

PESF Annual

130.00

$0.25 \quad 32.50$

570.00

142.50

\begin{tabular}{|c|c|c|c|c|c|}
\hline $\begin{array}{l}\text { Unit } \\
\text { Type }\end{array}$ & $\begin{array}{l}\text { Plant } \\
\text { Type }\end{array}$ & $\begin{array}{l}\text { Project } \\
\text { Status }\end{array}$ & $\begin{array}{l}\text { Dam } \\
\text { status }\end{array}$ & $\begin{array}{l}\text { Latitude } \\
\text { Longitude }\end{array}$ & $\begin{array}{l}4203.00 \\
7325.00\end{array}$ \\
\hline C & ROR & $X X$ & $\mathbf{U}$ & & \\
\hline
\end{tabular}

Factor

Exist Prob

Factor

Exist Prob

wild/Scenic Protection

wild/Scenic Tributary or Upstream/Downstream wild/Scenic Location Cultural value Fish Presence Value Geologic Value Historic Value other Value Recreation Value Scenic value

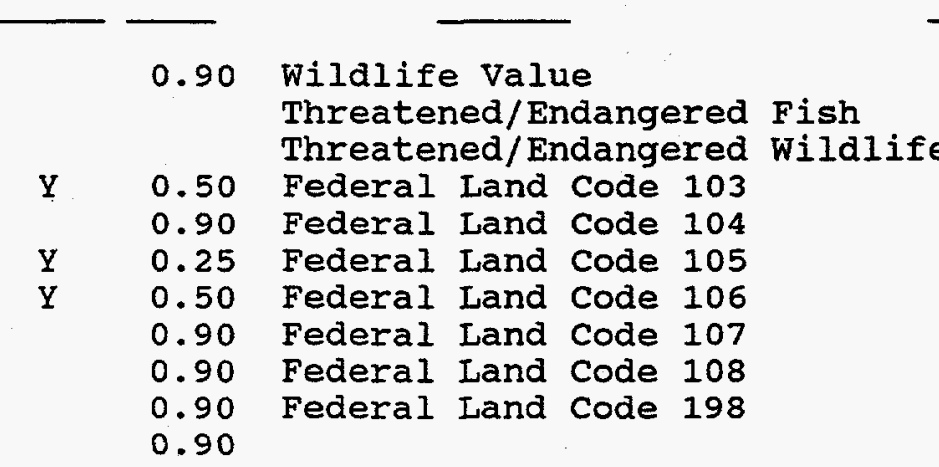


DATE: $06 / 27 / 95$

PAGE NO: 38

FERC

Number

09037

COLUMBIA MILL

County Name

BERKSHIRE

Class

Owner Name

R BURLINGTON ENERGY DEV ASSOC

Name Plate

Rating ( $\mathrm{KW}$ )

420.00
Annual Energy Rating (MWh)

1800.00

$$
\text { River Basin }
$$

HOUSATONIC RIVER BASIN
State

Name

MA

$\begin{array}{llllll}\text { Unit } & \text { Plant } & \text { Project } & \text { Dam } & \text { Latitude } & 4221.00 \\ \text { Type } & \text { Type } & \text { Status } & \text { Status } & \text { Longitude } & 7314.00\end{array}$

C

ROR

$\mathrm{XX}$

$\mathrm{U}$

\author{
Factor \\ Wild/Scenic Protection \\ wild/Scenic Tributary or \\ Upstream/Downstream \\ wild/Scenic Location \\ Cultural value \\ Fish Presence Value \\ Geologic Value \\ Historic Value \\ other value \\ Recreation value \\ Scenic Value
}

Exist Prob

0.90

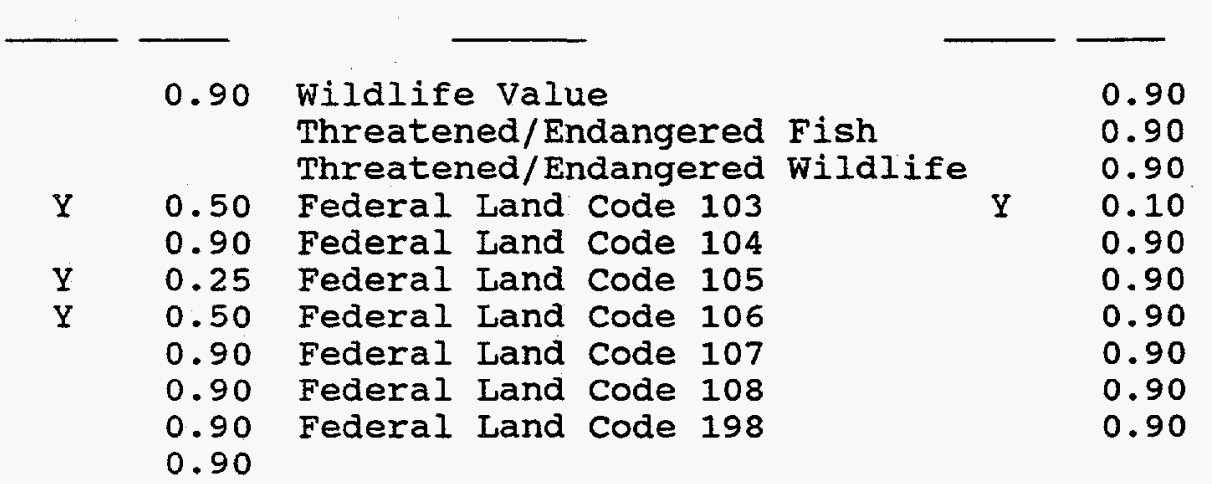

PESF Annual Energy Rating (MWh)

180 
RES O U R E D A T A B A S E L I S T I N G

DATE: $06 / 27 / 95$

PAGE NO: 39

FERC

Number

09039

GORDON DAM

county Name

BERKSHIRE

\section{Class}

Name Plate

Rating (KW)

$$
380.00
$$

R

BURLINGTON

PESF

$$
0.10 \quad 38.00
$$

Unit

$$
\begin{aligned}
& \text { Plant } \\
& \text { Type }
\end{aligned}
$$

C

ROR

Factor

Wild/Scenic Protection

wild/Scenic Tributary or Upstream/ Downstream wild/Scenic Location cultural value Fish Presence Value Geologic Value Historic Value other Value Recreation Value Scenic Value

XX

\begin{abstract}
Project status
\end{abstract}

Exist Prob

0.90

Wildlife Value Threatened/Endangered Fish Threatened/Endangered Wildlife

0.90

0.90

$Y \quad 0.25$

Federal Land Code 103

Federal Land Code 104

Federal Land Code 105

Annual Energy

Rating (MWh)

1600.00

Latitude

4222.00

status

Longitude

7315.00 state

Name

MA
PESF Annual Energy Rating (MWh)

160

Y 0.50 Federal Land Code 106

0.90 Federal Land Code 107

0.90 Federal Land Code 108

0.90 Federal Land Code 198

Exist Prob

0.90

0.90

0.90

0.90

0.10

0.90

0.90

0.90

0.90

0.90

0.90 
FERC

Number

09040 GORDON

County Name

WORCESTER
Plant Name

Class

\section{$\mathbf{R}$}

BURLINGTON

Name Plate

Rating ( $\mathrm{KW}$ )

$$
25.00
$$

PESF*KW
State

Name

MA
River Basin

THAMES RIVER BASIN

LITTLE R, FRENCH R

$\begin{array}{ccclll}\text { Unit } & \text { Plant } & \text { Project } & \text { Dam } & \text { Latitude } & 4207.00 \\ \text { Type } & \text { Type } & \text { Status } & \text { Status } & \text { Longitude } & 7154.00 \\ \text { C } & \text { ROR } & \text { XX } & \text { WO } & \end{array}$

Factor

Exist Prob

0.90

Wild/Scenic Protection wild/Scenic Tributary or Upstream/Downstream wild/Scenic Location Cultural value Fish Presence Value Geologic Value Historic Value other value

Recreation Value Scenic Value
Factor

Exist Prob

Annual Energy

110.00

99 Energy Rating (MWh) 
DATE: $06 / 27 / 95$

FERC

Number

Plant Name

09066

CONWAY

county Name

FRANKLIN

Class Owner Name

R BURLINGTON ENERGY DEV ASSOC

Name Plate

Rating (KW)

350.00

PESF

Annual Energy Rating (MWh)

1500.00

Latitude

Longitude

4232.00

Type

Type

status

$X X$

status

wo
750 state

Name

MA
PESF Annual Energy Rating (MWh)
C

Factor

wild/Scenic Protection

wild/Scenic Tributary or Upstream/ Downstream wild/Scenic Location Cultural value

Fish Presence Value

Geologic Value

Historic Value

other Value

Recreation Value

Scenic Value
Exist Prob

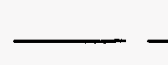

0.90

Wildlife Value

Threatened/Endangered Fish

Threatened/Endangered Wildlife

0.90 Federal Land code 103

0.90 Federal Land code 104

Y 0.75 Federal Land Code 105

0.90 Federal Land code 106

$Y \quad 0.75$ Federal Land Code 107

$Y \quad 0.75$ Federal Land Code 108

0.90 Federal Iand Code 198

0.90 
DATE: $06 / 27 / 95$

PAGE NO: 42

FERC

Number

Plant Name

09081 CENTER POND

County Name

BERKSHIRE
State

stream

E BR HOUSATONIC $\mathrm{R}$

MA
River Basin

HOUSATONIC RIVER BASIN

\section{Class Owner Name \\ R BURLINGTON ENERGY DEV ASSOC}

Name Plate Rating (KW) PESF
Annual Energy PESF*KW Rating (MWh)
PESF Annual Energy Rating (MWh)

$$
130.00 \quad 0.75 \quad 97.50
$$$$
570.00
$$$$
427.50
$$

$\begin{array}{ll}\text { Unit } & \text { Plant } \\ \text { Type } & \text { Type }\end{array}$

C

ROR

$$
\begin{aligned}
& \text { Project } \\
& \text { status }
\end{aligned}
$$

$\mathrm{XX}$

$\begin{array}{lll}\text { Dam } & \text { Latitude } & 4228.00 \\ \text { Status } & \text { Longitude } & 7309.00\end{array}$

WO
Factor

Exist Prob

wild/Scenic Protection wild/Scenic Tributary or Upstream/Downstream wild/scenic Location Cultural value

Fish Presence Value

Geologic Value

Historic Value

other Value

Recreation Value

Scenic Value
Exist Prob

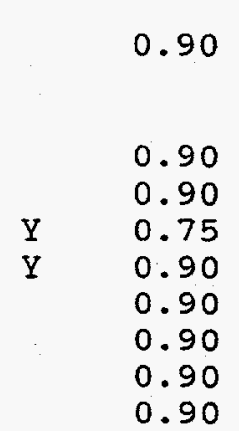

\section{Factor}

Exist Prob

Wildlife Value
Threatened/Endangered Fish
Threatened/Endangered Wildlife
Federal Land Code 103
Federal Land Code 104
Federal Land code 105
Federal Land Code 106
Federal Land Code 107
Federal Land Code 108
Federal Land Code 198

0.90

0.90

0.90

0.90

0.90

0.90

0.90

0.90

0.90

0.90 
R E S O U R C E

DATE: $06 / 27 / 95$

FERC

Number

09082 DAITON NO 1

county Name

BERKSHIRE
D A T A B A S E

PAGE NO: 43

L I S I N G

State

Name

E BR HOUSATONIC $\mathbf{R}$

MA

$$
\text { River Basin }
$$

HOUSATONIC RIVER BASIN

Class Owner Name

R BURLINGTON ENERGY DEV ASSOC

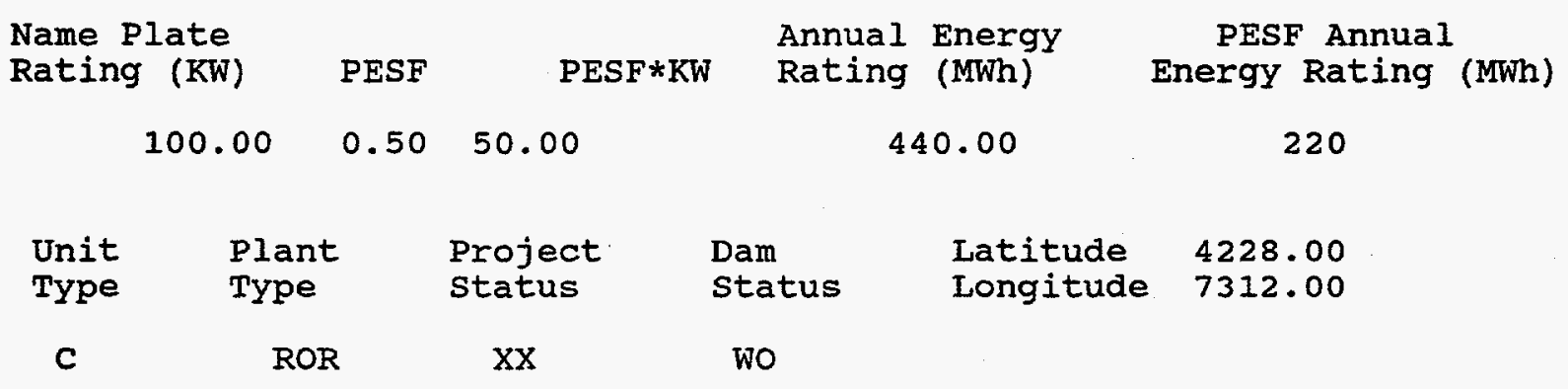

Factor

Exist Prob

Factor

Exist Prob

Wild/Scenic Protection

Wild/Scenic Tributary or Upstream/Downstream wild/Scenic Location Cultural value

Fish Presence Value

Geologic Value

Historic Value

other Value

Recreation Value

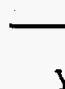

$Y \quad 0.50$

Wildlife Value

Threatened/Endangered Fish

0.90

Threatened/Endangered Wildlife

0.90

0.90

$Y \quad 0.75$ Federal Land Code 103

0.90

0.90 Federal Land Code 104

0.90

$Y \quad 0.75$ Federal Land code 105

0.90

$\mathrm{Y} \quad 0.90$ Federal Land Code 106

0.90

0.90 Federal Land Code 107

0.90

$\mathrm{Y} \quad 0.75$ Federal Land code 108

0.90

0.90

Federal Land Code 198

0.90 
DATE: $06 / 27 / 95$

FERC

Number

Plant Name

09083 DALTON NO 6

county Name

BERKSHIRE

class Owner Name

$R$ BURLINGTON ENERGY DEV ASSOC

River Basin

HOUSATONIC RIVER BASIN
State

Name

E BR HOUSATONIC $\mathrm{R}$

MA

PAGE NO: 44

stream

BASIN

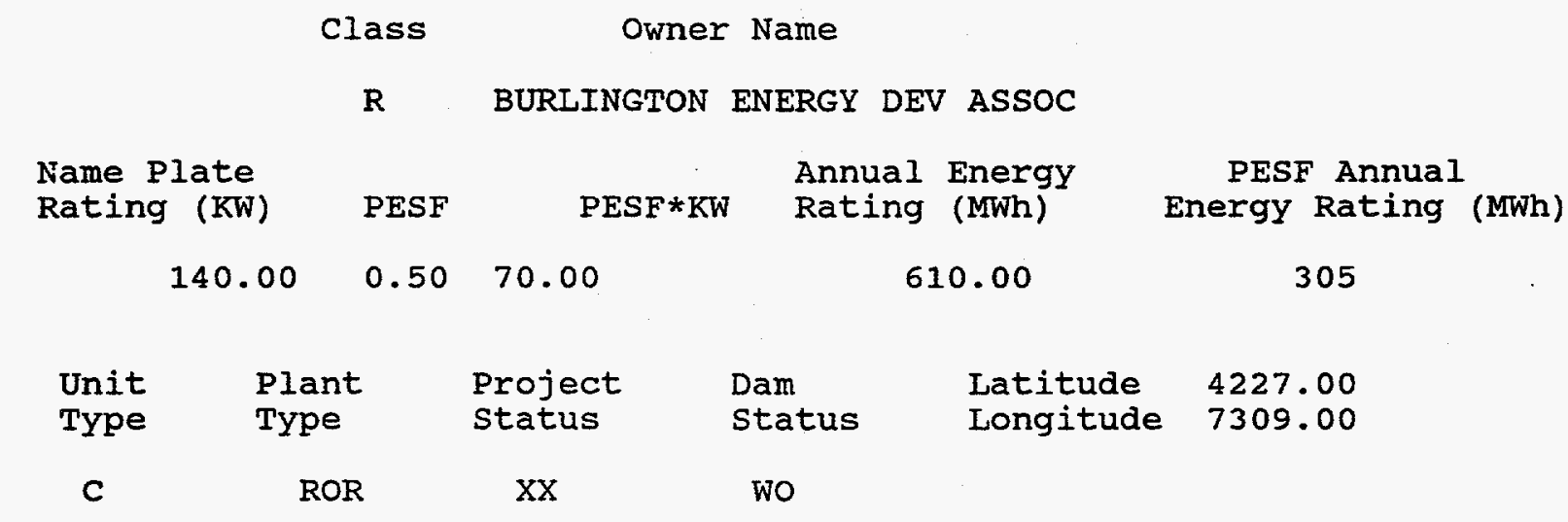

Factor

Wild/Scenic Protection

Wild/Scenic Tributary or Upstream/Downstream wild/scenic Location Cultural value

Fish Presence Value

Geologic Value

Historic Value

other Value

Recreation value

scenic Value
Exist Prob

Factor

Exist Prob

$\mathrm{Y}$

0.50

Wildife Value

Threatened/Endangered Fish

Threatened/Endangered Wildiife

Y 0.75 Federal Land Code 103

0.90 Federal Land code 104

$\mathrm{Y} \quad 0.75$ Federal Land code 105

Y 0.90 Federal Land code 106

0.90 Federal Land Code 107

Y 0.75 Federal Land code 108

Federal Land code 198
Exist Prob

0.90

0.90

0.90

0.90

0.90

0.90

0.90

0.90

0.90

0.90 

R E S O U R C E
D A T A B A S E
L I S T I N G

DATE: $06 / 27 / 95$

PAGE NO: 45

FERC

Number

09129

OTIS

Plant Name

stream

FALL R, W BR FARMINGTON $R$

River Basin

BERKSHIRE

Class Owner Name

R BURLINGTON ENERGY DEV ASSOC

Name Plate Rating (KW)

PESF PESF*KW

Annual Energy Rating (MWh)

2200.00

Latitude

4209.00

$\begin{array}{ll}\text { Unit } & \text { Plant } \\ \text { Type } & \text { Type } \\ \text { C } & \end{array}$

Project status

$\mathrm{XX}$

Dam
status

Longitude

7304.00 state Name

MA
PESF Annual Energy Rating (MWh)
Factor

wild/Scenic Protection

wild/Scenic Tributary or Upstream/Downstream wild/Scenic Location Cultural Value

Fish Presence Value Geologic Value Historic Value other Value Recreation Value Scenic Value

\section{Exist Prob}

Y

$$
0.10
$$

Wildife Value Threatened/Endangered $\mathrm{Fish}$ Threatened/Endangered wildlife

Exist Prob

Y 0.50 Federal Land code 103

0.90 Federal Land code 104

$Y \quad 0.25$ Federal Land code 105

$Y \quad 0.50$ Federal Land code 106

0.90 Federal Land code 107

0.90 Federal Land code 108

0.90 Federal Land code 198

0.90
0.90

0.90

0.90

0.90

0.90

0.90

0.90

0.90

0.90

0.90 
FERC

Number

09255
Plant Name

WESTVILLE

county Name

WORCESTER

Class

Owner Name

$\mathrm{R}$ SOUTHBRIDGE ASSOC

Name Plate

Rating (KW)

1000.00

PESF

PESF $* K W$

Annual Energy

Rating (MWh)

4300.00

0.90900 .00

Project status

XX

Exist Prob

Plant

Type

ROR

C

\section{Dam \\ status}

wo
State

Name

MA

\author{
Factor \\ wild/Scenic Protection \\ wild/Scenic Tributary or \\ Upstream/Downstream \\ wild/Scenic Location \\ Cultural value \\ Fish Presence Value \\ Geologic Value \\ Historic Value \\ other value \\ Recreation value \\ Scenic Value
}

Factor

Latitude

Longitude
PESF Annual Energy Rating (MWh)

3870

$\begin{array}{ll}0.90 & \text { Wildlife Value } \\ & \text { Threatened/Endangered Fish } \\ \text { Threatened/Endangered Wildlife } \\ 0.90 & \text { Federal Land Code } 103 \\ 0.90 & \text { Federal Land Code } 104 \\ 0.90 & \text { Federal Land Code } 105 \\ 0.90 & \text { Federal Land Code } 106 \\ 0.90 & \text { Federal Land Code } 107 \\ 0.90 & \text { Federal Land Code } 108 \\ 0.90 & \text { Federal Land Code } 198\end{array}$

Exist Prob

4204.00

7204.00 
DATE: $06 / 27 / 95$

PAGE NO: 47

FERC

Number

Plant Name

Stream

State

09276

COOKS

MILL R

Name

\section{6}

county Name

HAMPSHIRE

River Basin

CONNECTICUT RIVER BASIN

\section{Class Owner Name \\ R LEEDS POWER CO}

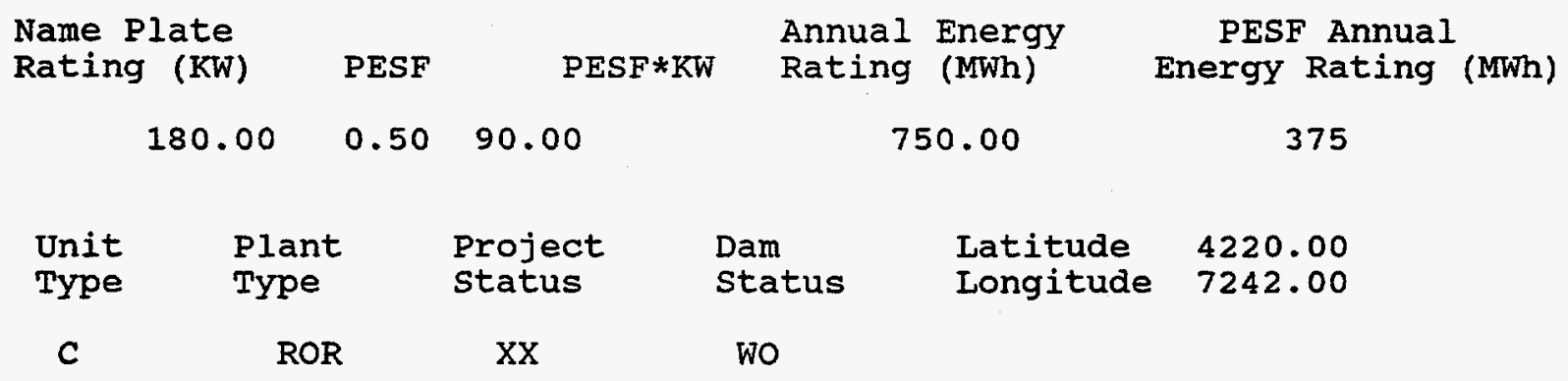

\section{Factor}

wild/Scenic Protection

wild/Scenic Tributary or Upstream/Downstream wild/Scenic Location cultural value

Fish Presence Value

Geologic Value

Historic Value

other value

Recreation Value

Scenic Value
Exist Prob

Factor

Exist Prob

.

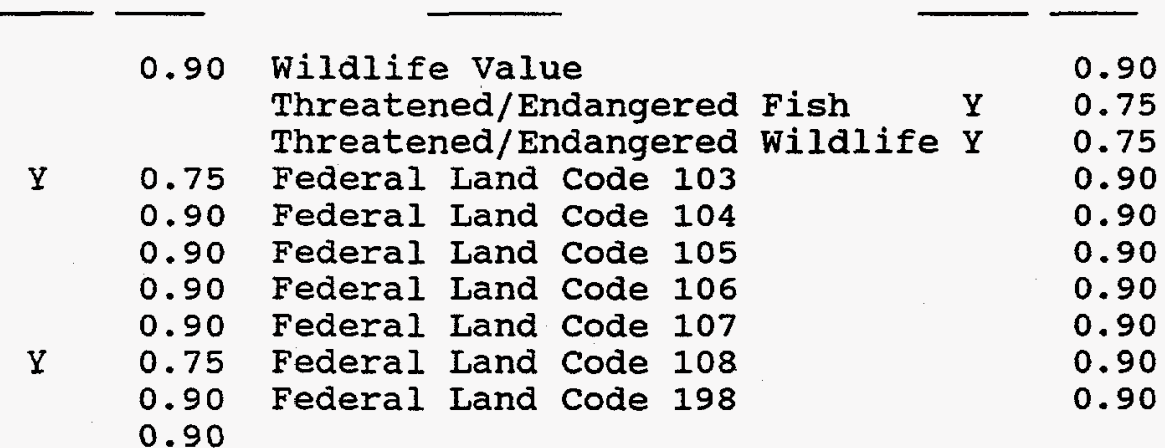


FERC

Number

09287
Plant Name county Name

MIDDLESEX
State

Stream

SUDBURY R

MA

River Basin

MERRIMACK RIVER BASIN

\author{
Class \\ Owner Name \\ R BURLINGTON ENERGY DEV ASSOC
}

Name Plate
Rating (KW) PESF

60.00

$0.50 \quad 30.00$

Plant

Type

Project status

$\mathrm{XX}$

Exist Prob

\section{Dam \\ Status}

WO
Factor
PESF Annual Energy Rating (MWh)
Factor

ROR

C

Wild/scenic Protection

wild/Scenic Tributary or Upstream/Downstream wild/Scenic Location Cultural value Fish Presence Value Geologic Value Historic Value other Value Recreation Value Scenic Value
Exist Prob

$Y \quad 0.50$

Wildiife Value Threatened/Endangered Fish

130

0.90

0.90

Y 0.75 Federal Land code 103

wildlife

0.90

0.90 Federal Land Code 104

0.90

$\mathrm{Y} \quad 0.75$

Federal Land Code 105

0.90

0.90 Federal Land Code 106

0.90

0.90 Federal Land Code 107

0.90

0.90 Federal Land Code 108

0.90

$\mathrm{Y} \quad 0.75$

Federal Land Code 198

0.90

0.90

0.90 

R E S O U R C E
D A T A B A S E
L I S T I N G

DATE: $06 / 27 / 95$

PAGE NO: 49

FERC

Number

09288

SAXONVILLE

county Name

MIDDLESEX

\section{Class}

Owner Name

R BURLINGTON ENERGY DEV ASSOC

Name Plate Rating (KW)

PESF

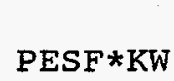

$0.50 \quad 75.00$

150.00

Project status

$\mathrm{XX}$
Annual Energy Rating (MWh)

650.00 state Name

MA

River Basin

MERRIMACK RIVER BASIN

PESF Annual Energy Rating (MWh)

325

$\begin{array}{ccclll}\text { Unit } & \text { Plant } & \text { Project } & \text { Dam } & \text { Latitude } & 4220.00 \\ \text { Type } & \text { Type } & \text { Status } & \text { Status } & \text { Longitude } & 7124.0 .0 \\ \text { C } & \text { ROR } & \text { XX } & \text { wo } & \end{array}$

\section{Factor}

Exist Prob

Factor

Exist Prob

wild/scenic Protection

wild/Scenic Tributary or Upstream/Downstream wild/Scenic Location Cultural value

Fish Presence Value

Geologic Value

Historic Value

other Value

Recreation Value

Scenic Value

$\mathrm{Y}$

$$
0.50
$$

Wildlife Value Threatened/Endangered Fish Threatened/Endangered Wildlife

0.90

0.90

0.90

$Y$

0.75 Federal Land code 103

0.90 Federal Iand code 104

$Y \quad 0.75$ Federal Land code 105

0.90 Federal Land code 106

0.90 Federal Land code 107

0.90 Federal Land Code 108

$Y \quad 0.75$ Federal Land code 198
0.90

0.90

0.90

0.90

0.90

0.90

0.90 
FERC

Number

Plant Name

09329

LEEDS

county Name

HAMPSHIRE
Stream

MILL R

River Basin

CONNECTICUT RIVER BASIN
State

Name

MA

Class owner Name

$R$ BURLINGTON ENERGY DEV ASSOC
Name Plate Rating (KW) PESF PESF*KW
Annual Energy Rating (MWh) 75.00 $0.75 \quad 56.25$

325.00

Latitude Longitude

4221.00

Project

XX

\begin{tabular}{|c|c|c|c|c|c|}
\hline $\begin{array}{l}\text { Unit } \\
\text { Type }\end{array}$ & $\begin{array}{l}\text { Plant } \\
\text { Type }\end{array}$ & $\begin{array}{l}\text { Project } \\
\text { status }\end{array}$ & $\begin{array}{l}\text { Dam } \\
\text { Status }\end{array}$ & $\begin{array}{l}\text { Latitude } \\
\text { Longitude }\end{array}$ & $\begin{array}{l}4221.00 \\
7242.00\end{array}$ \\
\hline C & ROR & $\mathrm{XX}$ & WO & & \\
\hline
\end{tabular}

PESF Annual Energy Rating (MWh)

Factor

Exist Prob

Factor

Exist Prob

Wild/scenic Protection

wild/Scenic Tributary or Upstream/Downstream

wild/scenic Location Cultural value

Fish Presence Value

Geologic Value

Historic Value

Other value

Recreation Value

Scenic Value

\subsection{0}

Wildlife Value

Threatened/Endangered Fish

Threatened/Endangered Wildlife

0.90

0.90

$Y$

0.75 Federal Land Code 103

0.90 Federal Land code 104

0.90 Federal Land Code 105

0.90 Federal Land code 106

0.90 Federal Land code 107

0.90 Federal Land code 108

0.90 Federal Land code 198

0.90

0.90

0.90

0.90

0.90

0.90

0.90

0.90

0.90 
RES O URCE D A T A B A S E I I S T I N G

DATE: $06 / 27 / 95$

PAGE NO: 51

FERC

Number

Plant Name

09330 NONOTUCKET STREET

County Name

HAMPSHIRE

Class Owner Name

R . BURLINGTON ENERGY DEV ASSOC

Name Plate

Rating ( $\mathrm{KW}$ )

PESF

Annual Energy

75.00

$0.75 \quad 56.25$

$\mathrm{PESF} * \mathrm{KW}$

Rating (MWh)

330.00

Latitude

Longitude

4220.00

$\begin{array}{ccccc}\text { Unit } & \text { Plant } & \text { Project } & \text { Dam } & \text { Latitude } \\ \text { Type } & \text { Type } & \text { Status } & \text { Status } & \text { Longitude } \\ \text { C } & \text { ROR } & \text { XX } & \text { WO } & \end{array}$

Factor

Exist Prob

wild/Scenic Protection

wild/Scenic Tributary or Upstream/Downstream wild/Scenic Location Cultural Value

Fish Presence Value

Geologic Value

Historic Value

other Value

Recreation Value

Scenic Value

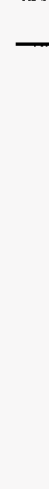

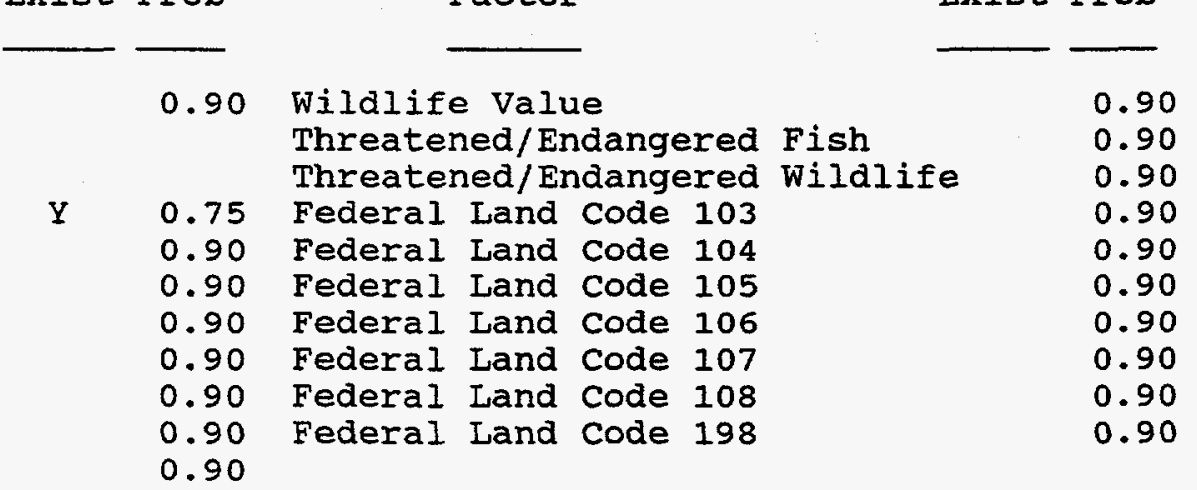

PESF Annual Energy Rating (MWh)

247.50

State

Name

MA 
DATE: $06 / 27 / 95$

PAGE NO: 52

FERC

Number

Plant Name

09331
SPRING STREET

County Name

HAMPSHIRE
Stream

MILL R

River Basin

CONNECTICUT RIVER BASIN
State

Name

MA

Class Owner Name

R BURLINGTON ENERGY DEV ASSOC

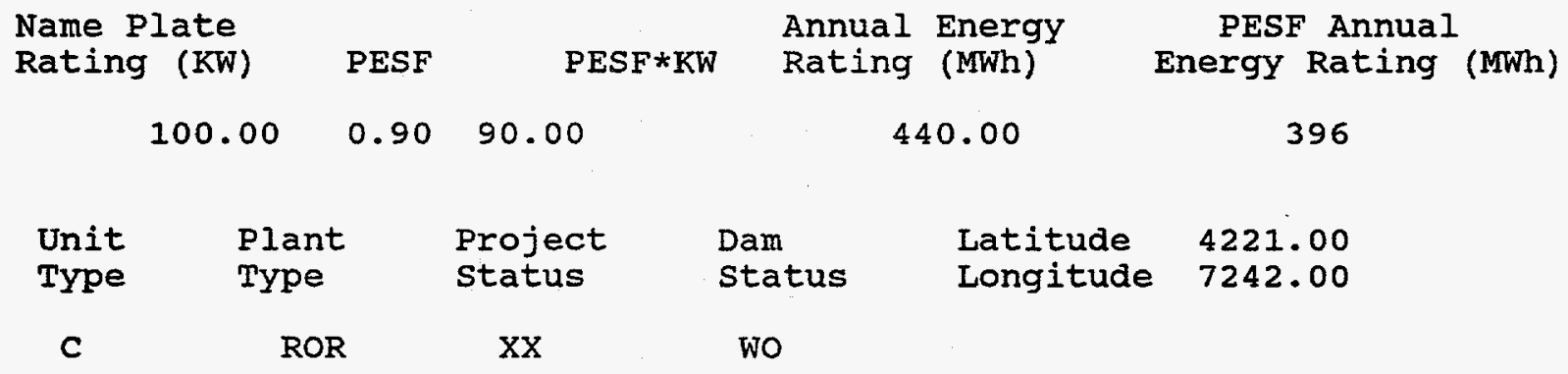

Factor

Exist Prob

Factor

Exist Prob

wild/Scenic Protection

Wild/Scenic Tributary or

0.90 Wildlife Value Threatened/Endangered Fish

0.90 Upstream/Downstream Threatened/Endangered Wildlife

0.90

wild/Scenic Location

0.90

Federal Land code 103

0.90

0.90 Federal Land Code 104

0.90

Cultural Value

Fish Presence Value

0.90 Federal Land Code 105

0.90

0.90 Federal Land Code 106

0.90

0.90 Federal Land Code 107

0.90

Historic Value

0.90 Federal Land Code 108

0.90

other Value

Recreation Value

0.90

Federal Land code 198

0.90

Scenic Value

0.90

0.90 
RESO URCE D A T A B A S E I I S T I N G

DATE: $06 / 27 / 95$

PAGE NO: 53

FERC

Number

Plant Name

stream

State

09332

PARADISE

MILL $\mathrm{R}$

Name

09332

county Name

River Basin

HAMPSHIRE

CONNECTICUT RIVER BASIN

\author{
Class Owner Name \\ R BURLINGTON ENERGY DEV ASSOC
}

$\begin{array}{lll}\text { Name Plate } & & \text { Annual Energy } \\ \text { Rating (KW) } & \text { PESF } & \text { PESF*KW } \\ \text { Rating (MWh) }\end{array}$

$75.00 \quad 0.50 \quad 37.50$

\begin{tabular}{|c|c|c|c|c|}
\hline $\begin{array}{l}\text { Unit } \\
\text { Type }\end{array}$ & $\begin{array}{l}\text { Plant } \\
\text { Type }\end{array}$ & $\begin{array}{l}\text { Project } \\
\text { status }\end{array}$ & $\begin{array}{l}\text { Dam } \\
\text { Status }\end{array}$ & $\begin{array}{l}\text { Latitude } \\
\text { Longitude }\end{array}$ \\
\hline C & ROR & $\mathrm{XX}$ & wo. & \\
\hline
\end{tabular}

Factor

Exist Prob

Factor

Exist Prob

wild/Scenic Protection

wild/Scenic Tributary or Upstream/Downstream wild/Scenic Location Cultural value

Fish Presence Value

Geologic Value

Historic Value

other Value

Recreation Value

Scenic Value

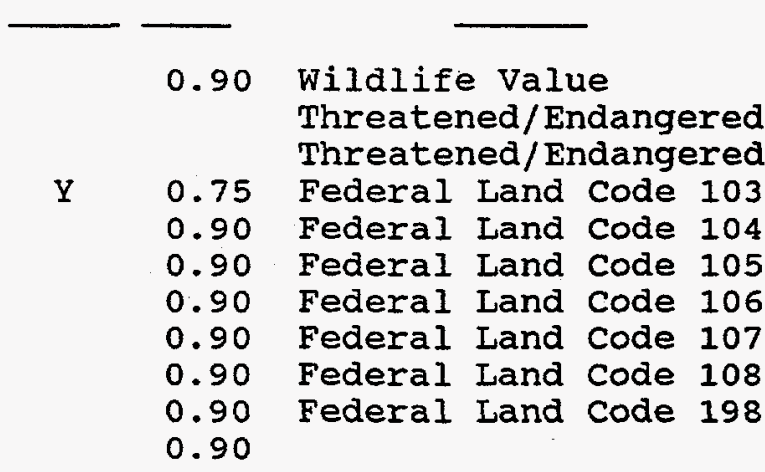

PESF Annual Energy Rating (MWh)

162.50

MA 
DATE: $06 / 27 / 95$

\section{FERC}

Number

Plant Name

09333

COCHRANE

county Name

NORFOLK

\section{Class}

Owner Name

R BURLINGTON ENERGY DEV ASSOC

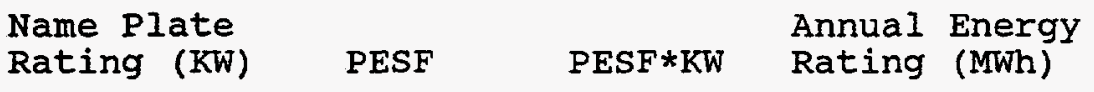

$$
200.00 \quad 0.50 \quad 100.00
$$

Type

Plant

Type
C

ROR
State

Name

MA
Exist Prob

Factor

Wild/Scenic Protection wild/Scenic Tributary or Upstream/Downstream wild/Scenic Location Cultural Value Fish Presence Value Geologic Value Historic Value other value Recreation value Scenic Value
800.00

Latitude

Longitude

4215.00

status.

$X X$

Status

wo

Factor

Exist Prob

$$
0.90
$$

Wildlife Value Threatened/Endangered Fish

PESF Annual Energy Rating (MWh)

$$
400
$$

7116.00

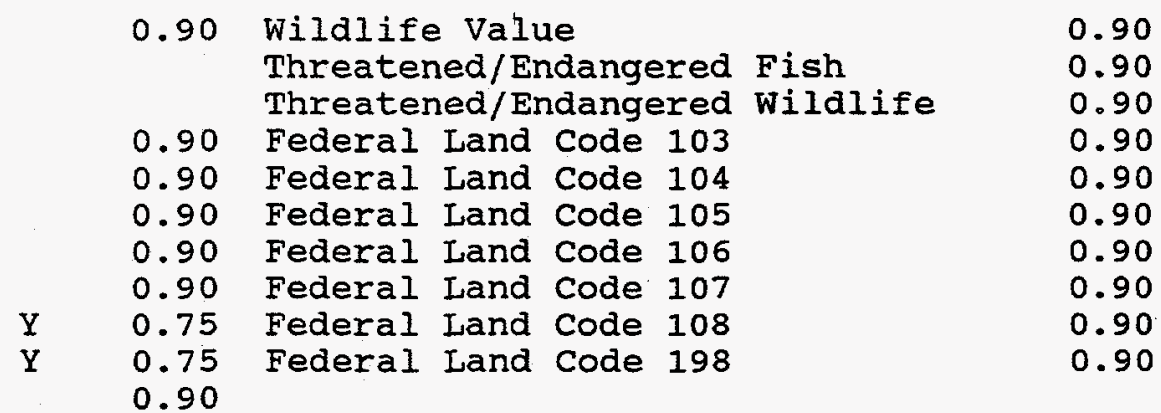



R E S O U R C E
D A T A B A S E
L I S T I N G

DATE: $06 / 27 / 95$

PAGE NO: 55

FERC

Number

Plant Name

Stream

State

Name

09432 MANHAN

MANHAN $R$

MA

county Name

HAMPSHIRE
River Basin

CONNECTICUT RIVER BASIN

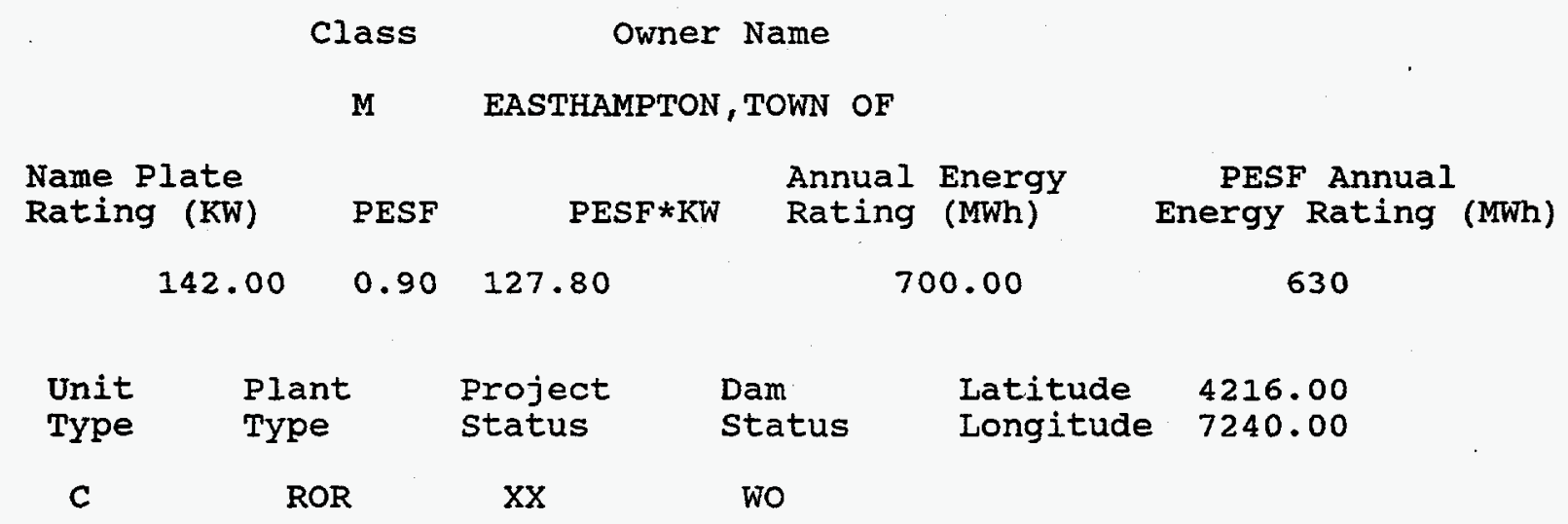

Factor

wild/Scenic Protection wild/Scenic Tributary or Upstream/Downstream wild/Scenic Location Cultural value Fish Presence Value Geologic Value Historic Value other Value Recreation Value Scenic Value
Exist Prob

0.90

Wildlife Value Threatened/Endangered Fish Threatened/Endangered Wildlife

Exist Prob

Factor

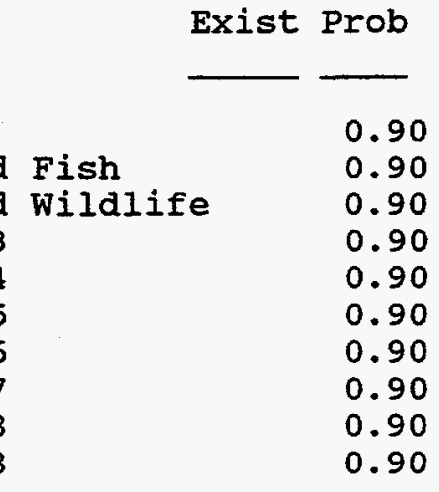


DATE: $06 / 27 / 95$

PAGE NO: 56

FERC

Number

Plant Name

09486 MOODY ST (WALTHAM)

county Name

MIDDLESEX stream

CHARLES $R$

River Basin

MINOR RIVER BASINS
State

Name

MA

Class Owner Name

R WALTHAM HYDRO CONSVS

Name Plate

PESF

Annual Energy

Rating ( $K W$ )

PESF

PESF*KW

Rating (MWh)

PESF Annual

364.00

$0.50 \quad 182.00$

1800.00

$\begin{array}{ll}\text { Unit } & \text { Plant } \\ \text { Type } & \text { Type }\end{array}$

C

ROR
Factor

Wild/Scenic Protection wild/Scenic Tributary or Upstream/Downstream wild/Scenic Location Cultural value Fish Presence Value Geologic Value Historic Value other Value Recreation Value Scenic Value
Project status

$\mathrm{XX}$
Dam status
Iatitude Longitude

WO
Exist Prob

Factor
4222.00

7114.00
900

Energy Rating (MWh) 
RESOURCE D A T A B A S E I I S T I N G

DATE: $06 / 27 / 95$

PAGE NO: 57

FERC

Number

09606

QUEQUECHAN RIVER

County Name

BRISTOL

Class

$\mathbf{R}$

BURLINGTON ENERGY DEV ASSOC

Name Plate Rating (KW)

PESF

Annual Energy Rating (MWh)

$$
500.00 \quad 0.90 \quad 450.00
$$

PESF *KW

2000.00
River Basin

MINOR RIVER BASINS state

Name

MA

$\begin{array}{ccclll}\text { Unit } & \text { Plant } & \text { Project } & \text { Dam } & \text { Latitude } \\ \text { Type } & \text { Type } & \text { Status } & \text { Status } & \text { Longitude } 7109.00 \\ \text { C } & \text { DIV } & \mathrm{XX} & \mathrm{U} & \end{array}$

\section{Factor}

Wild/Scenic Protection Wild/Scenic Tributary or Upstream/Downstream wild/Scenic Location Cultural value

Fish Presence Value Geologic Value Historic Value other Value Recreation Value Scenic Value
Exist Prob

0.90 Wildlife Value Threatened/Endangered Fish Threatened/Endangered Wilalife Exist Prob Factor

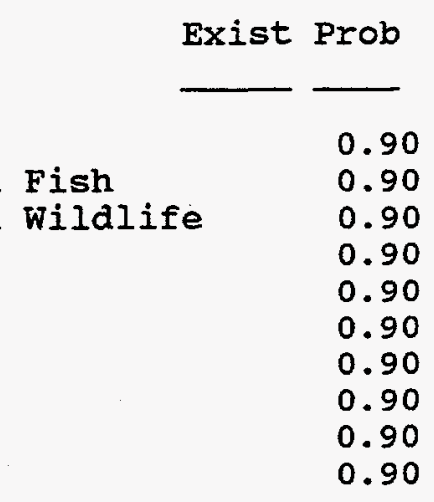

0.90 Federal Land Code 103

0.90 Federal Land code 104

0.90 Federal Land code 105

0.90 Federal Land code 106

0.90 Federal Land code 107

0.90 Federal Land Code 108

0.90 Federal Land Code 198

0.90
PESF Annual Energy Rating (MWh)

1800 
FERC

Number

Plant Name
County Name

HAMPDEN
CHICOPEE R

stream
State

Name

MA
River Basin

CONNECTICUT RIVER BASIN $\begin{array}{cc}\text { Class } & \text { Owner Name } \\ \text { R } & \text { SWIFT RIVER/HAFSLUND CO }\end{array}$

Name Plate Rating (KW) PESF $\mathrm{PESF} * \mathrm{KW}$

Annual Energy Rating (MWh)

2360.00 $0.50 \quad 1180.00$

Project status

$\mathrm{XX}$

$$
13500.00
$$

Latitude Longitude
PESF Annual Energy Rating (MWh)

$\begin{array}{ccclll}\text { Unit } & \text { Plant } & \text { Project } & \text { Dam } & \text { Latitude } & 4210.00 \\ \text { Type } & \text { TYpe } & \text { Status } & \text { Status } & \text { Longitude } & 7222.00 \\ \text { C } & \text { ROR } & \text { XX } & \text { WO } & \end{array}$

Factor

Exist Prob

Factor

Exist Prob

wild/Scenic Protection wild/Scenic Tributary or Upstream/Downstream wild/Scenic Location Cultural value Fish Presence Value Geologic Value Historic Value other Value Recreation Value Scenic Value

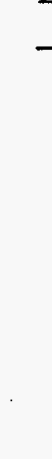

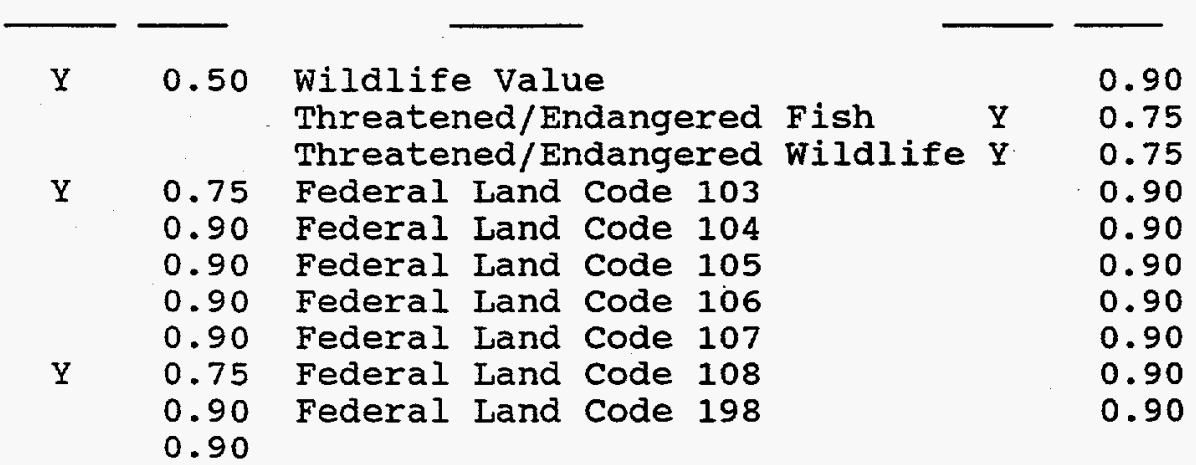


R E S URCE D A T A B A E L I S T I N G

DATE: $06 / 27 / 95$

PAGE NO: 59

FERC

Number

Plant Name

Stream

State

09616 DELANEY

SQUANNACOOK $R$

Name

09616 DELANEY

county Name

River Basin

MIDDLESEX

MERRIMACK RIVER BASIN

Class Owner Name

R DELANEY, JOHN M ET AL

\begin{tabular}{|c|c|c|c|c|c|c|}
\hline \multicolumn{2}{|c|}{$\begin{array}{l}\text { Name Plate } \\
\text { Rating (KW) }\end{array}$} & PESF & PESF $* K W$ & \multicolumn{2}{|c|}{$\begin{array}{l}\text { Annual Energy } \\
\text { Rating (MWh) }\end{array}$} & $\begin{array}{l}\text { PESF Annual } \\
\text { Energy Rating (MWh) }\end{array}$ \\
\hline & 105.00 & 0.90 & 94.50 & & 82.00 & 253.80 \\
\hline $\begin{array}{l}\text { Unit } \\
\text { Type }\end{array}$ & $\begin{array}{l}\mathrm{Pl} \\
\mathrm{TYl}\end{array}$ & & $\begin{array}{l}\text { Project } \\
\text { status }\end{array}$ & $\begin{array}{l}\text { Dam } \\
\text { status }\end{array}$ & $\begin{array}{l}\text { Latitude } \\
\text { Longitude }\end{array}$ & $\begin{array}{l}4240.00 \\
7146.00\end{array}$ \\
\hline c & & & $\mathrm{XX}$ & $\mathrm{U}$ & & \\
\hline
\end{tabular}

Factor

wild/Scenic Protection

Wild/Scenic Tributary or

Upstream/Downstream

wild/Scenic Location

Cultural value

Fish Presence Value

Geologic Value

Historic Value

Other Value

Recreation Value

Scenic Value
Exist Prob

$$
0.90
$$$$
0.90 \text { Wildife Value }
$$$$
\text { Threatened/Endangered Fish }
$$$$
\text { Threatened/Endangered Wildife }
$$

0.90 Federal Land code 103

0.90 Federal Land code 104

0.90 Federal Land Code 105

0.90 Federal Land Code 106

0.90 Federal Land Code 107

0.90 Federal Land Code 108

0.90 Federal Land Code 198

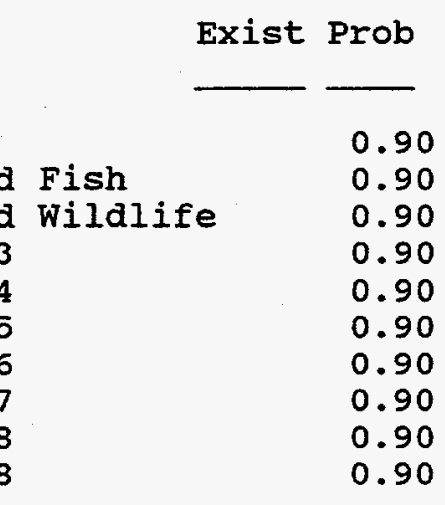

0.90

0.90

0.90

0.90

0.90

0.90

0.90
MA 
FERC

Number

Plant Name

09640

TEXTILE UPPER

county Name

HAMPSHIRE stream

SWIFT R

River Basin

CONNECTICUT RIVER BASIN state

Name

MA

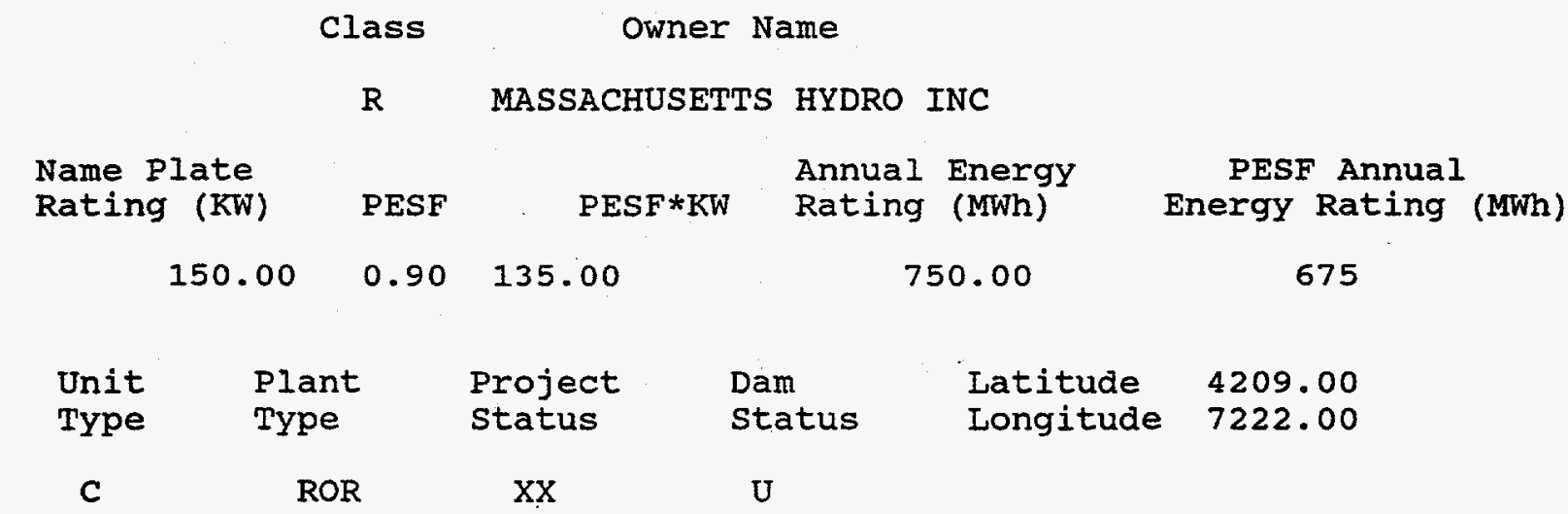

Factor

Wild/Scenic Protection Wild/Scenic Tributary or Upstream/Downstream wild/Scenic Iocation Cultural value

Fish Presence Value

Geologic Value

Historic Value

Other Value

Recreation Value

Scenic Value
Exist Prob

Factor

Exist Prob

0.90

Wildlife Value

Threatened/Endangered Fish

Threatened/Endangered Wildlife

0.90

0.90

0.90

0.90 Federal Land Code 103

0.90 Federal Land code 104

0.90

0.90

Federal Land code 105

0.90 Federal Land Code 106

0.90 Federal Land Code 107

0.90 Federal Land code 108

0.90 Federal Land code 198

0.90
0.90

0.90

0.90

0.90

0.90

0.90 
R E S UR C E D A T A B A S E I I S T I N G

DATE: $06 / 27 / 95$

PAGE NO: 61

FERC

Number

Plant Name

stream

State

09720 TEXTILE LOWER

SWIFT R

Name

county Name

River Basin

HAMPDEN

CONNECTICUT RIVER BASIN

Class Owner Name

R BONDSVILLE HYDROPOWER INC

Name plate

Rating $(\mathrm{KW})$

PESF PESF* KW Annual Energy

PESF Annual

150.00

$0.90 \quad 135.00$

750.00

Energy Rating (MWh)

675

$\begin{array}{ccclll}\text { Unit } & \text { Plant } & \text { Project } & \text { Dam } & \text { Latitude } & 4212.00 \\ \text { Type } & \text { Type } & \text { Status } & \text { Status } & \text { Longitude } & 7220.00 \\ \text { C } & \text { ROR } & \text { XX } & \text { U } & \end{array}$

Factor

wild/Scenic Protection

wild/Scenic Tributary or Upstream/Downstream wild/Scenic Location cultural value

Fish Presence Value

Geologic Value

Historic Value

other value

Recreation Value

Scenic Value
Exist Prob

0.90

Wildlife Value

Threatened/Endangered Fish

Threatened/Endangered Wildlife

0.90 Federal Land Code 103

0.90 Federal Land Code 104

0.90 Federal Land Code 105

0.90 Federal Iand code 106

0.90 Federal Land Code 107

0.90 Federal Land Code 108

0.90 Federal Land code 198

0.90
MA

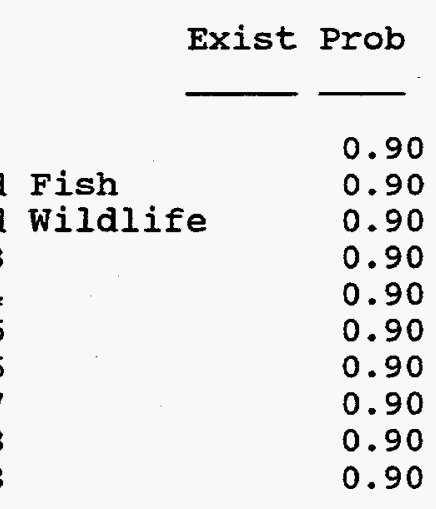


DATE: $06 / 27 / 95$

PAGE NO: 62

FERC

Number

09895
Plant Name

KNIGHTVILLE

County Name

HAMPSHIRE
State

Name

WESTFIELD $R$

MA
River Basin

CONNECTICUT RIVER BASIN
Class Owner Name

R KNIGHTVILLE DAM ASSOC

$\begin{array}{lccc}\begin{array}{l}\text { Name Plate } \\ \text { Rating (KW) }\end{array} & \text { PESF } & \text { PESF*KW } & \begin{array}{c}\text { Annual Energy } \\ \text { Rating }\end{array} \\ 3000.00 & 0.50 & 1500.00 & 9437.00\end{array}$

PESF Annual Energy Rating (MWh)

4718.50

$\begin{array}{cccll}\text { Unit } & \text { Plant } & \text { Project } & \text { Dam } & \text { Latitude } \\ \text { Type } & \text { Type } & \text { Status } & \text { Status } & \text { Longitude } 7254.00 \\ \text { C } & \text { STG } & \text { XX } & \text { Wo } & \end{array}$

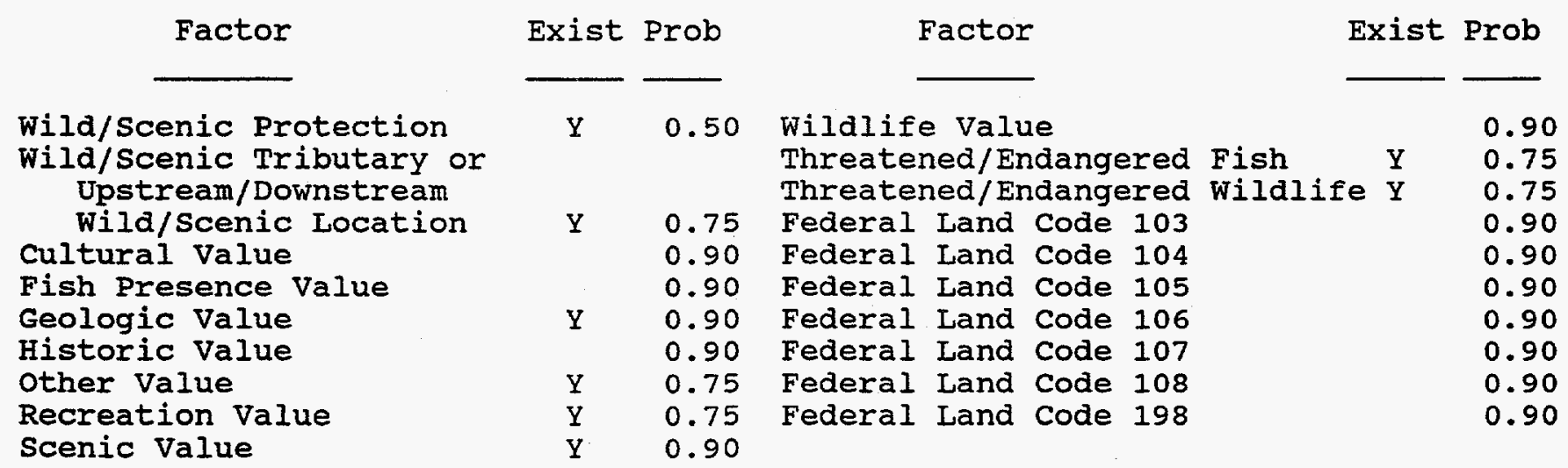


RES O UR C E D A T A B A S E L I S T I N G

DATE: $06 / 27 / 95$

PAGE NO: 63

FERC

Number

Plant Name

09978 LAKE GARDNER

county Name

ESSEX

Class

Owner Name

R ESSEX TURBINE CO INC

Name Plate
Rating (KW) PESF $\quad$ PESF*KW

120.00

0.7590 .00

600.00

PESF Annual Energy Rating (MWh)

450 state Name

MA
River Basin

MERRIMACK RIVER BASIN

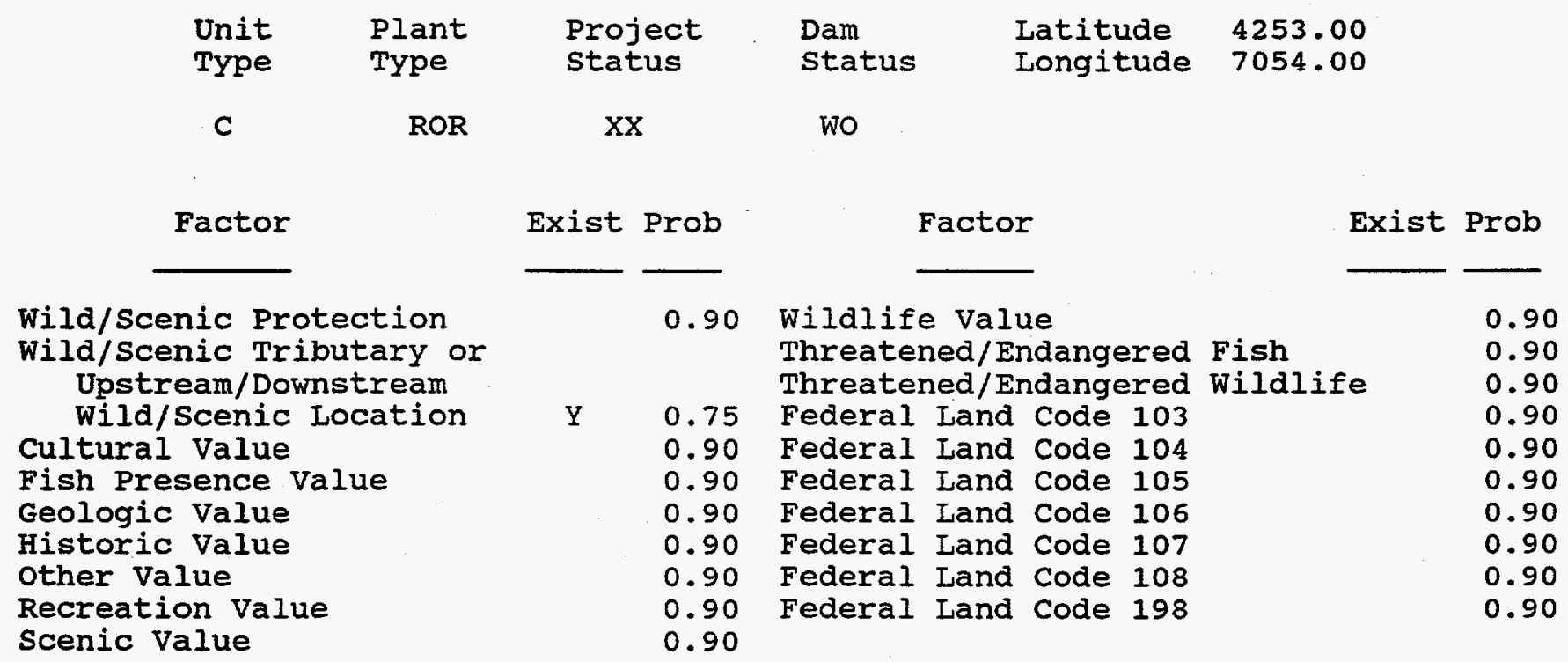


FERC

Number

Plant Name

10012 WASHINGTON STREET

County Name

MIDDLESEX stream

ASSABET $R$

River Basin

MERRIMACK RIVER BASIN
State

Name

MA

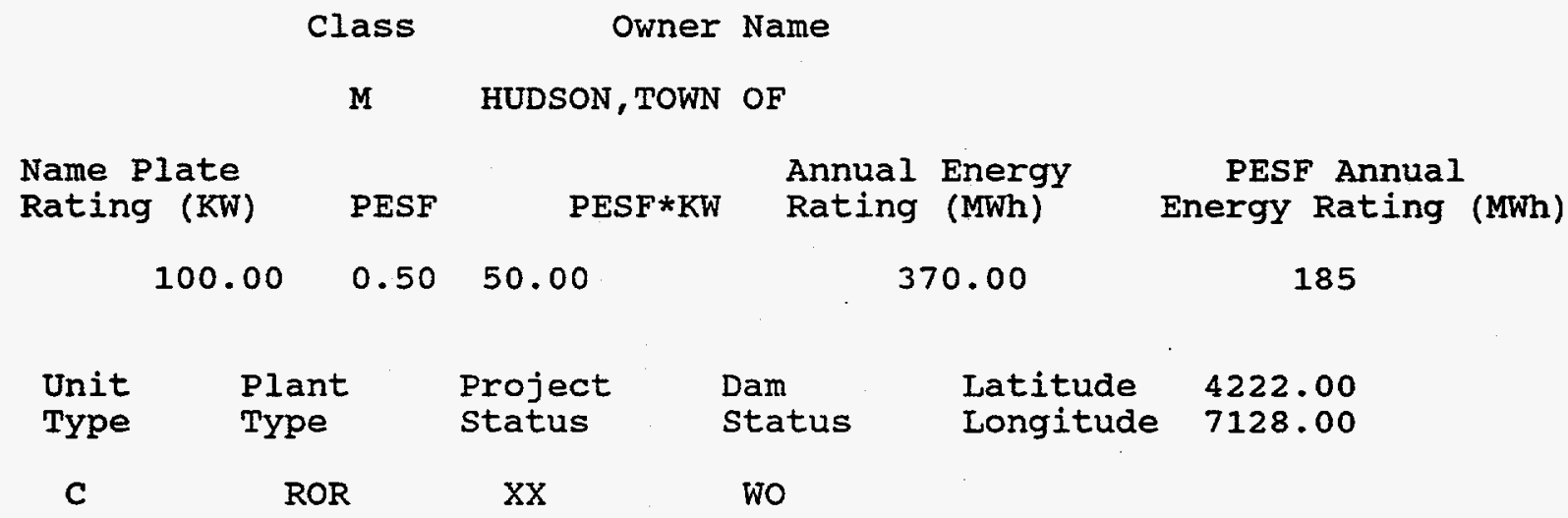

Factor

Exist Prob

$Y$

0.50

wild/Scenic Tributary or Upstream/Downstream wild/Scenic Location Cultural value

Fish Presence Value

Geologic Value

Historic Value

Other Value

Recreation Value

Scenic Value

0.90
Factor

Exist Prob

Wildife Value

Threatened/Endangered Fish Threatened/Endangered Wildlife

0.90 Federal Land code 103

0.90 Federal Land Code 104

0.90 Federal Iand code 105

0.90 Federal Land code 106

0.90 Federal Land Code 107

0.90 Federal Land code 108

0.90 Federal Land Code 198
0.90

0.90

0.90

0.90

0.90

0.90

0.90

0.90

0.90

0.90 
RESOURCE D A T A B A S E I I S T I N G

DATE: $06 / 27 / 95$

PAGE NO: 65

FERC

Number

10032

CROCKER MILL

county Name

HAMPDEN
Plant Name

HOLYOKE CNL (CONN R)

River Basin

CONNECTICUT RIVER BASIN

\section{Class Owner Name}

I IINWEAVE, INC

Name Plate PESF

Annual Energy Rating ( $\mathrm{KW}$ )

PEST

PESF*KW Rating (MWh)

$$
1937.00
$$

state

Name

MA

$$
\begin{array}{lll}
575.00 & 0.90 \quad 517.50
\end{array}
$$

$\begin{array}{ccclll}\text { Unit } & \text { Plant } & \text { Project } & \text { Dam } & \text { Latitude } & 4212.00 \\ \text { Type } & \text { Type } & \text { Status } & \text { Status } & \text { Longitude } & 7236.00 \\ \text { C } & \text { ROR } & \text { XX } & \text { U } & \end{array}$

\section{Factor}

Wild/Scenic Protection wild/Scenic Tributary or Upstream/Downstream Wild/Scenic Location Cultural value Fish Presence Value Geologic Value Historic Value other Value Recreation Value Scenic Value
Exist Prob

0.90

Wildlife Value Threatened/Endangered Fish Threatened/Endangered Wildilfe

0.90 Federal Land Code 103

0.90 Federal Land Code 104

0.90 Federal Land code 105

0.90 Federal Land code 106

0.90 Federal Land Code 107

0.90 Federal Land Code 108

0.90 Federal Land Code 198

0.90
PESF Annual Energy Rating (MWh)

1743.30 
DATE: $06 / 27 / 95$

PAGE NO: 66

FERC

Number

10054
Plant Name

MOTHER BK PWR MUSEUM

county Name

NORFOLK
River Basin

MINOR RIVER BASINS
State

Name

MA

Class Owner Name

I BALANCE ONE INC ET AL

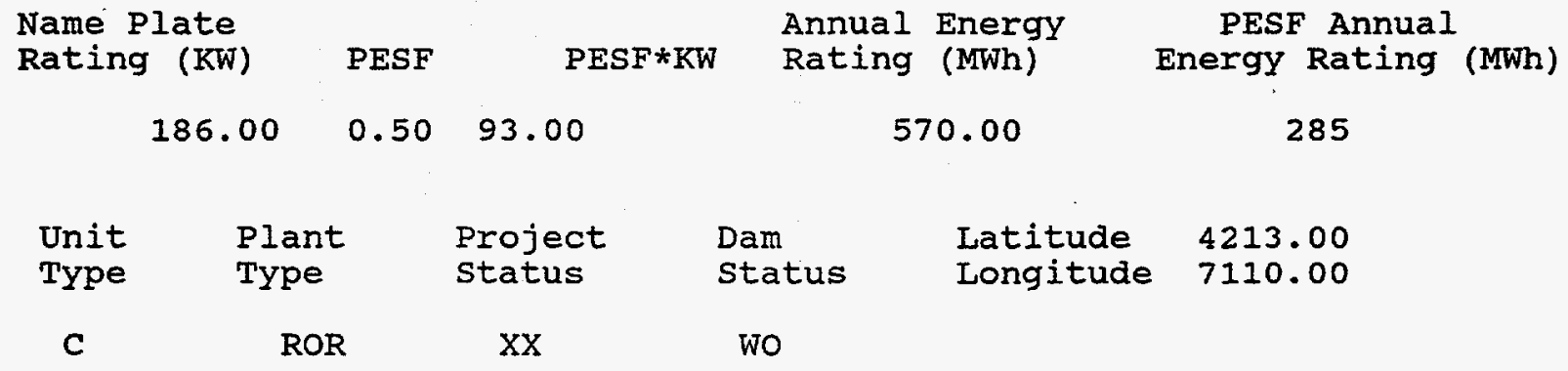

Factor

Exist Prob

Factor

Exist Prob

id/Scenic Protection

wild/Scenic Tributary or Upstream/Downstream wild/Scenic Location Cultural value Fish Presence Value

Geologic Value

Historic Value

other value

Recreation Value

Scenic Value

$\begin{array}{lll}\text { Y } & 0.50 & \begin{array}{l}\text { Wildife Value } \\ \text { Threatened/Endangered Fish }\end{array} \\ & & \text { Threatened/Endangered Wildife } \\ \text { Y } & 0.75 & \text { Federal Land Code } 103 \\ & 0.90 & \text { Federal Land Code } 104 \\ & 0.90 & \text { Federal Land Code } 105 \\ & 0.90 & \text { Federal Land Code } 106 \\ & 0.90 & \text { Federal Land Code } 107 \\ & 0.75 & \text { Federal Land Code } 108 \\ & 0.90 & \text { Federal Land Code } 198 \\ & 0.90 & \end{array}$

0.90

0.90

0.90

0.90

0.90

0.90

0.90

0.90

0.90

0.90 

R E S O U R C E
D A $T A B A S E$
I I S T I N G

DATE: $06 / 27 / 95$

PAGE NO: 67

FERC

Number

10121A CIRCULAR DAM

MIDDLESEX
Plant Name

\author{
county Name
}

Class Owner Name

R BALANCE ONE INC

\begin{tabular}{|c|c|c|c|c|c|c|c|}
\hline $\begin{array}{l}\text { Name } \\
\text { Rating }\end{array}$ & $\begin{array}{l}\text { late } \\
\text { (KW) }\end{array}$ & PESF & PESF*KW & \multicolumn{2}{|c|}{$\begin{array}{l}\text { Annual Energy } \\
\text { Rating (MWh) }\end{array}$} & \multicolumn{2}{|c|}{$\begin{array}{l}\text { PESF Annual } \\
\text { Energy Rating (MWh) }\end{array}$} \\
\hline & 275.00 & 0.50 & 137.50 & \multicolumn{2}{|c|}{1060.00} & 530 & \\
\hline $\begin{array}{l}\text { Unit } \\
\text { Type }\end{array}$ & $\begin{array}{l}\text { Pl } \\
\text { TY }\end{array}$ & & $\begin{array}{l}\text { Project } \\
\text { status }\end{array}$ & $\begin{array}{l}\text { Dam } \\
\text { status }\end{array}$ & $\begin{array}{l}\text { Latitude } \\
\text { Longitude }\end{array}$ & $\begin{array}{l}4219.00 \\
7114.00\end{array}$ & \\
\hline C & & & $\mathrm{XX}$ & wo & & & \\
\hline
\end{tabular}

\section{CHARLES R}

River Basin

MINOR RIVER BASINS
Exist Prob

Factor

Wild/Scenic Protection

wild/scenic Tributary or Upstream/Downstream wild/Scenic Location cultural value Fish Presence Value Geologic Value Historic Value other Value Recreation Value Scenic Value state Name

MA 
FERC

Number

10121B SILK MILL

county Name NORFOLK
Plant Name

CHARLES R

River Basin

MINOR RIVER BASINS
State

Name

MA

Class Owner Name

$R$ BALANCE ONE INC

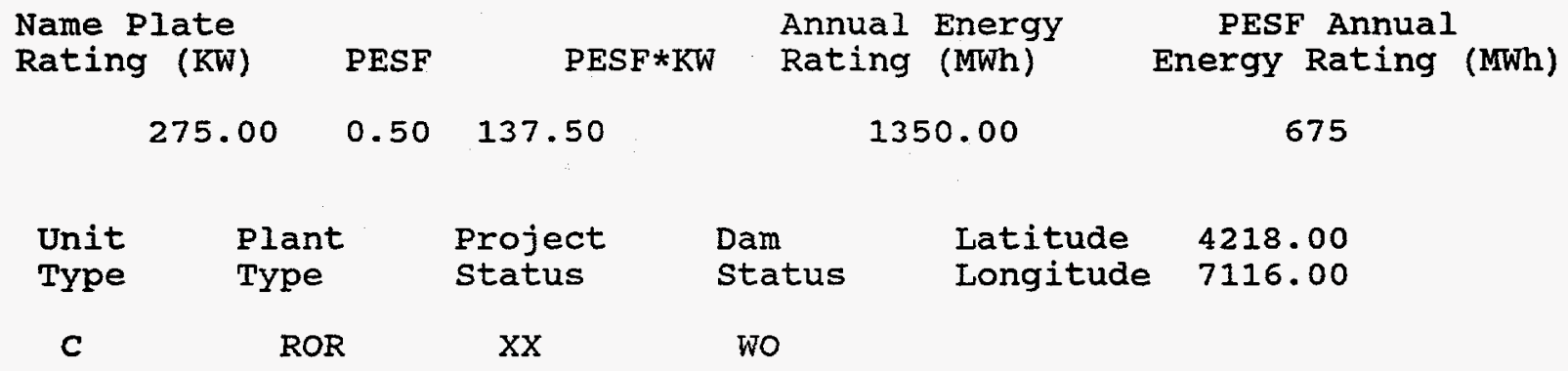

Factor

Exist Prob

Factor

wild/scenic Protection

wild/Scenic Tributary or Upstream/Downstream wild/Scenic Location Cultural Value

Fish Presence Value

Geologic Value

Historic Value other value

Recreation Value

Exist Pri

0.90

Wildife Value Threatened/Endangered Fish Threatened/Endangered Wildlife

0.90 Federal Land code 103

0.90 Federal Land code 104

0.90 Federal Land Code 105

0.90 Federal Land code 106

0.90 Federal Land code 107

$Y \quad 0.75$ Federal Land code 108

$Y \quad 0.75$ Federal Land code 198
Exist Prob

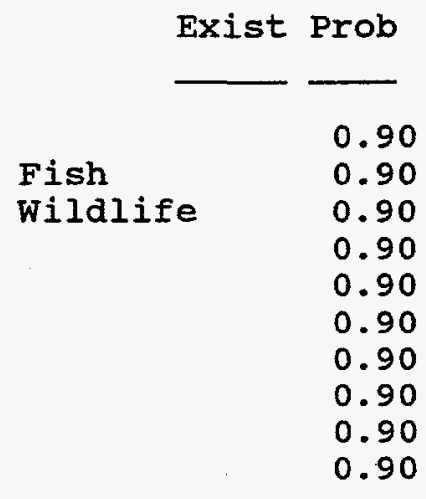

0.90

0.90

0.90

0.90

0.90

0.90 

R E S O U R C E
D A T A B A S E
I I S T I N G

DATE: $06 / 27 / 95$

PAGE NO: 69

FERC

Number

10177
Plant Name

SHAKER MILL

County Name

BERKSHIRE
River Basin

HOUSATONIC RIVER BASIN
Class Owner Name

$M$ WEST STOCKBRIDGE, TOWN OF
Name Plate Rating ( $\mathrm{KW}$ )

30.00

PESF

0.50

15.00

Project status

I.E
Annual Energy Rating (MWh)

161.00 state Name

MA

$\begin{array}{ccclll}\text { Unit } & \text { Plant } & \text { Project } & \text { Dam } & \text { Latitude } & 4216.00 \\ \text { Type } & \text { Type } & \text { Status } & \text { Status } & \text { Longitude } & 7321.00 \\ \text { C } & \text { ROR } & \text { LE } & \text { WO } & \end{array}$

Factor

wild/Scenic Protection wild/Scenic Tributary or Upstream/Downstream wild/Scenic Location Cultural value

Fish Presence Value

Geologic Value

Historic Value

Other Value

Recreation Value

Scenic Value
Exist Prob

0.90

Wildlife Value Threatened/Endangered Wildlife Y

Y 0.75 Federal Land Code 103

0.90 Federal Land Code 104

0.90 Federal Land code 105

0.90 Federal Land code 106

0.90 Federal Land Code 107

0.90 Federal Land code 108

0.90 Federal Land Code 198

0.90
PESF Annual Energy Rating (MWh)

80.50

0.90


FERC

Number

Plant Name

10338 WEST GROTON

County Name

MIDDLESEX

Class

Owner Name

R LONGHILI ASSOC

Name Plate Rating (KW)

210.00

PESF

PESF*KW

Annual Energy

Rating (MWh)

PESF Annual

$0.90 \quad 189.00$

900.00

810 state Name

MA
Project status

$\mathrm{XX}$
WO

\begin{tabular}{|c|c|c|c|c|c|}
\hline $\begin{array}{l}\text { Unit } \\
\text { Type }\end{array}$ & $\begin{array}{l}\text { Plant } \\
\text { Type }\end{array}$ & $\begin{array}{l}\text { Project } \\
\text { status }\end{array}$ & $\begin{array}{l}\text { Dam } \\
\text { status }\end{array}$ & $\begin{array}{l}\text { Latitude } \\
\text { Longitude }\end{array}$ & $\begin{array}{l}4241.00 \\
7146.00\end{array}$ \\
\hline C & ROR & $\mathrm{XX}$ & Wo & & \\
\hline
\end{tabular}

Factor

wild/Scenic Protection Wild/Scenic Tributary or Upstream/Downstream wild/Scenic Location Cultural value Fish Presence Value Geologic Value Historic Value other value Recreation value Scenic Value
Exist Prob

0.90

Wildife Value Threatened/Endangered Fish Threatened/Endangered Wildlife

0.90 Federal Land code 103

0.90 Federal Land Code 104

0.90 Federal Land Code 105

0.90 Federal Land Code 106

0.90 Federal Land Code 107

0.90 Federal Land Code 108

0.90 Federal Land Code 198

0.90
Exist Prob

0.90

0.90

0.90

0.90

0.90

0.90

0.90

0.90

0.90

0.90 
R E S O U R E D A T A B A S E I I S T I N G

DATE: $06 / 27 / 95$

PAGE NO: 71

FERC

Number

10563

GEO MAC MEMORIAL

MIDDLESEX

Plant Name

10563

\author{
county Name \\ GEO MAC MEMORIAL
}

MIDDLESEX

\section{Class}

R

Name Plate

Rating ( $\mathrm{KW}$ )

376.00
Stream

WAMESIT CNL

River Basin

MERRIMACK RIVER BASIN state

Name

MA
PESF Annual

Annual Energy

Rating (MWh)

1945.00

1750.50

$\begin{array}{ccclll}\text { Unit } & \text { Plant } & \text { Project } & \text { Dam } & \text { Latitude } & 4237.00 \\ \text { Type } & \text { Type } & \text { Status } & \text { Status } & \text { Longitude } & 7118.00 \\ \text { C } & \text { DIV } & \text { XX } & \text { WO } & \end{array}$

\section{Factor}

Wild/scenic Protection

wild/Scenic Tributary or Upstream/Downstream wild/Scenic Location Cultural value Fish Presence Value Geologic Value Historic Value other Value Recreation Value Scenic Value
Exist Prob

0.90

wildlife Value Threatened/Endangered Fish Threatened/Endangered Wildife

0.90 Federal Land code 103

0.90 Federal Land code 104

0.90 Federal Land code 105

0.90 Federal Land code 106

0.90 Federal Land Code 107

0.90 Federal Land Code 108

0.90

0.90
Exist Prob

0.90

0.90

0.90

0.90

0.90

0.90

0.90

0.90

0.90

0.90 
R E S O U R E

DATE: $06 / 27 / 95$

FERC

Number

Plant Name

10580 POW WOW RIVER

County Name

ESSEX

Class

Owner Name

$R$ HEITMANN \& TINE ASSOC

\begin{tabular}{|c|c|c|c|c|c|c|}
\hline $\begin{array}{l}\text { Name } \\
\text { Ratin }\end{array}$ & $\begin{array}{l}\text { late } \\
(K W)\end{array}$ & PESF & $\mathrm{PESF} * \mathrm{KW}$ & $\begin{array}{l}\text { Annua } \\
\text { Ratin }\end{array}$ & $\begin{array}{l}\text { Energy } \\
(\text { MWh })\end{array}$ & $\begin{array}{l}\text { PESF Annual } \\
\text { Energy Rating (MWh) }\end{array}$ \\
\hline & 300.00 & 0.75 & 225.00 & & 00.00 & 1125 \\
\hline $\begin{array}{l}\text { Unit } \\
\text { Type }\end{array}$ & $\begin{array}{l}\text { Pla } \\
\text { TYI }\end{array}$ & & $\begin{array}{l}\text { Project } \\
\text { status }\end{array}$ & $\begin{array}{l}\text { Dam } \\
\text { status }\end{array}$ & $\begin{array}{l}\text { Latitude } \\
\text { Longitude }\end{array}$ & $\begin{array}{l}4251.00 \\
7054.00\end{array}$ \\
\hline C & & & $x x$ & wo & & \\
\hline
\end{tabular}

Factor

Exist Prob

Factor

Exist Prob

wild/Scenic Protection

Wild/Scenic Tributary or Upstream/ Downstream wild/Scenic Location Cultural Value Fish Presence value

Geologic Value Historic Value other value Recreation value Scenic Value
PAGE NO: 72

State

Name

MA

$$
\text { River Basin }
$$

MERRIMACK RIVER BASIN 
RESOURCE DA TABASE L I S T I N G

DATE: $06 / 27 / 95$

PAGE NO: 73

FERC

Number Plant Name

stream

State

10675

DWIGHT

CHICOPEE R

Name

10675

county Name

River Basin

HAMPDEN

CONNECTICUT RIVER BASIN

Class Owner Name

P WESTERN MASS ELECTRIC CO

Name Plate

PESF

Annual Energy

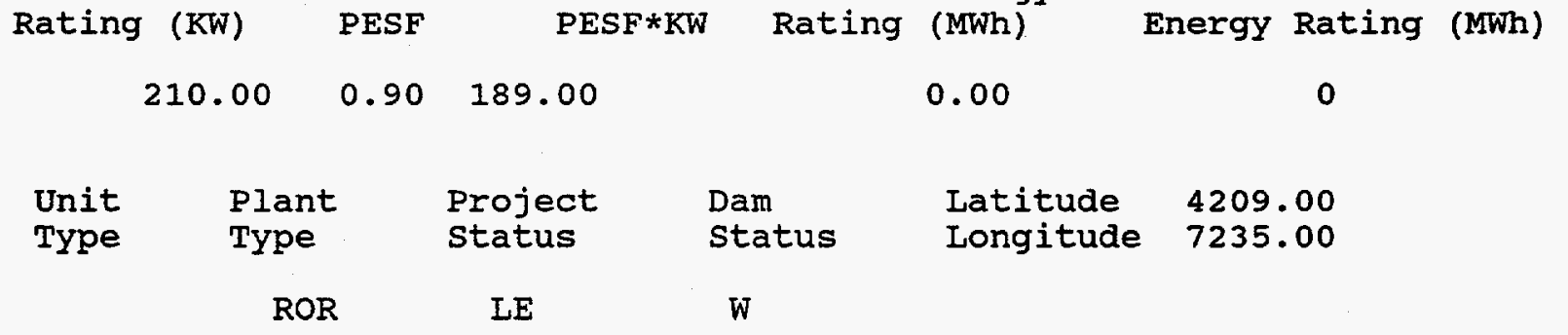

PESF Annual

Factor

Wild/Scenic Protection

wild/Scenic Tributary or

Upstream/Downstream

wild/Scenic Location

Cultural value

Fish Presence Value

Geologic Value

Historic Value

other Value

Recreation Value

Scenic value
Exist Prob

0.90 Wildlife Value

Threatened/Endangered $\mathrm{Fish}$

Threatened/Endangered Wildlife

0.90 Federal Land Code 103

0.90 Federal Land Code 104

0.90 Federal Land Code 105

0.90 Federal Land Code 106

0.90 Federal Land Code 107

0.90 Federal Land Code 108

0.90 Federal Land Code 198

0.90
Exist Prob

0.90

0.90

0.90

0.90

0.90

0.90

0.90

0.90

0.90

0.90 
DATE: $06 / 27 / 95$

PAGE NO: 74

FERC

Number

10676
Plant Name

RED BRIDGE

County Name

HAMPSHIRE
River Basin

CONNECTICUT RIVER BASIN
State

Name

MA

CHICOPEE R

stream

Name Plate

Rating ( $\mathrm{KW}$ )

PESF

Annual Energy

$\mathrm{PESF} * \mathrm{KW}$

Rating (MWh)

Energy Rating (MWh)

695.00

$0.90 \quad 625.50$

0.00

0

$\begin{array}{ll}\text { Unit } & \text { Plant } \\ \text { Type } & \text { Type }\end{array}$

Project status

Dam

status

Latitude

4211.00

ROR

LE

W

Factor

Exist Prob

Factor

Exist Prob

0.90

Wildife Value

Threatened/Endangered Fish

wild/Scenic Tributary or Threatened/Endangered Wildlife

0.90

0.90

Upstream/Downstream

wild/Scenic Location

0.90 Federal Land Code 103

0.90 Federal Land Code 104

0.90

Cultural value

Fish Presence Value

0.90

Federal Land Code 105

0.90 Federal Land code 106

0.90 Federal Land Code 107

0.90

Geologic Value

0.90

Federal Land Code 108

other Value

0.90

Federal Land Code 198

0.90

0.90

0.90

0.90

0.90

Recreation Value

0.90

0.90 
R E S O U R C E

DATE: $06 / 27 / 95$

FERC

Number

Plant Name

10677 PUTTS BRIDGE

county Name

HAMPDEN
D A T A B A $S$ E

PAGE NO: 75

state

Name

MA
CHICOPEE R

River Basin

CONNECTICUT RIVER BASIN
Class Owner Name

P WESTERN MASS ELECTRIC CO

Name Plate

Rating ( $K W$ )

PESF

Annual Energy

Rating (MWh)

0.00

Latitude

4210.00

Unit
Type

Plant

Type

Project
status

LE

Dam
status

Longitude

7229.00

0
ROR

C

Factor

Wild/Scenic Protection

wild/Scenic Tributary or Upstream/Downstream wild/Scenic Location Cultural value

Fish Presence Value

Geologic Value

Historic Value

other Value

Recreation Value

Scenic Value
Exist Prob

0.90

Wildlife Value

Threatened/Endangered Fish

Threatened/Endangered Wildlife

$\mathbf{Y}$

0.75

0.90

0.90

Federal Land code 103

Federal Land code 104

Federal Land code 105

0.90

Federal Land Code 106

0.90

Federal Land code 107

0.90

0.90

0.90

Federal Land code 198
PESF Annual Energy Rating (MWh)

Exist Prob

0.90

0.90

0.90

0.90

0.90

0.90

0.90

0.90

0.90

0.90 
R E S O U R C E

DATE: $06 / 27 / 95$
D A T A B A S E

L I S T I N G

PAGE NO: 76

\section{FERC}

Number

10678
Plant Name

INDIAN ORCHARD

County Name

HAMPDEN
State

Name

MA

CHICOPEE R

stream

River Basin

CONNECTICUT RIVER BASIN

Class owner Name

P WESTERN MASS ELECTRIC CO

Name Plate

Rating (KW)

PESF

Annual Energy

$0.75 \quad 322.50$

Rating (MWh)

PESF Annual Energy Rating (MWh)

\author{
430.00 \\ Unit Plant \\ Type Type \\ ROR
}

0.75
Project
Status

Dam

status

0.00

0

LE

W

Factor

wild/Scenic Protection

wild/Scenic Tributary or Upstream/Downstream

wild/scenic Location Cultural value

Fish Presence Value

Geologic Value

Historic Value

other value

Recreation Value

Scenic Value
Exist Prob

0.90

Y

0.75

0.90

0.90

0.90

0.90

0.90

0.90

0.90
Factor

Latitude Longitude

4210.00

7230.00 
RES O URCE D A T A B A E I I S T I N G

DATE: $06 / 27 / 95$

PAGE NO: 77

FERC

Number

10688
Plant Name

COSGROVE

County Name

WORCESTER

\author{
State \\ Name
}

MA
COSGROVE AQUE (WACHUSETT RES)

River Basin

MERRIMACK RIVER BASIN

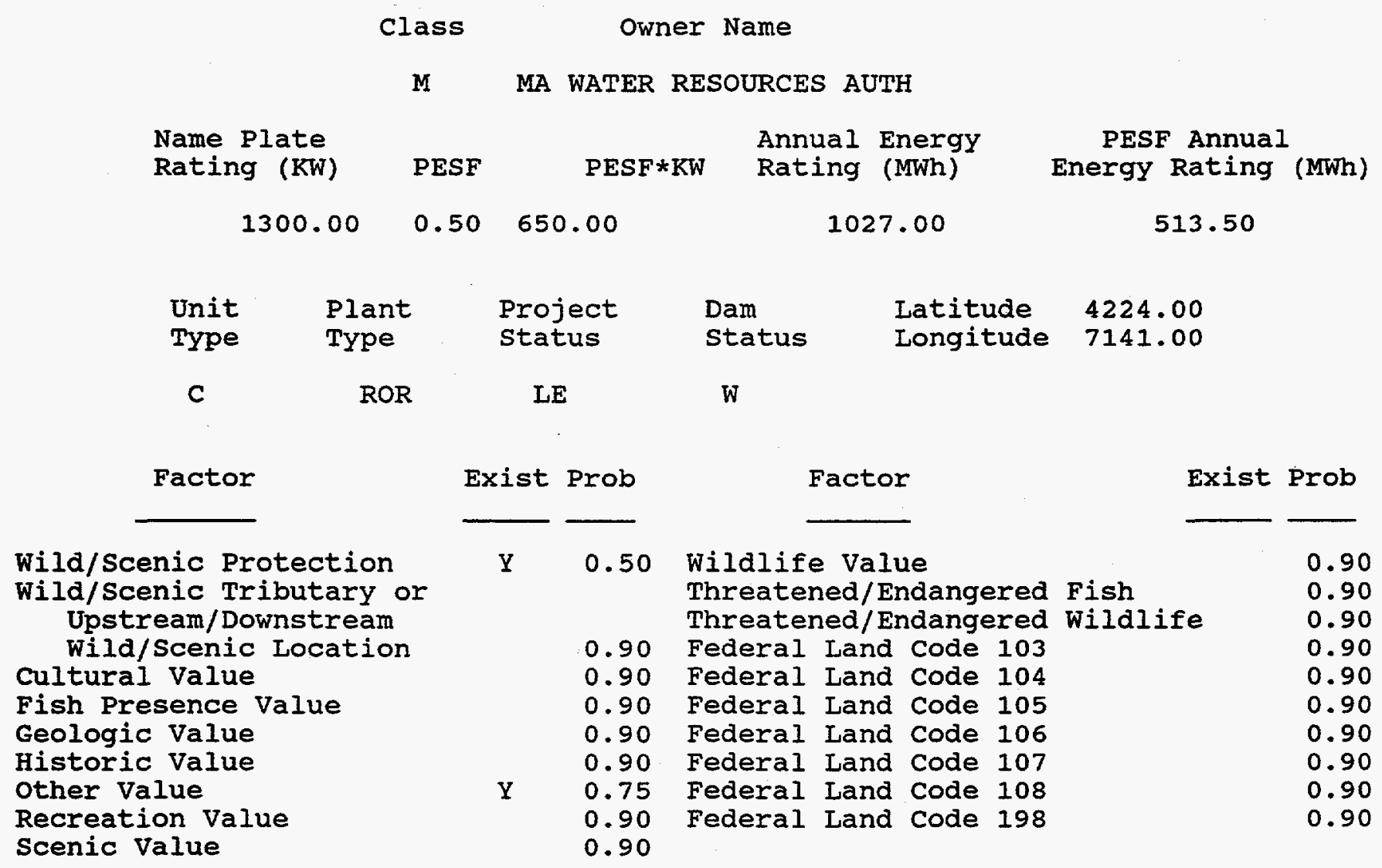


R E S O U R C E

DATE : $06 / 27 / 95$

\section{FERC}

Number

Plant Name

10806
STATION NO. 5

County Name

HAMPDEN
$D A T A B A S E$

I I S T N G

PAGE NO: 78
State

Name

MA
HOLYOKE CNL (CONN R)

River Basin

CONNECTICUT RIVER BASIN

\begin{tabular}{|c|c|c|c|c|c|}
\hline \multirow[b]{3}{*}{$\begin{array}{l}\text { Name Plate } \\
\text { Rating }(\mathrm{KW})\end{array}$} & lass & \multicolumn{2}{|c|}{ Owner Name } & & \multirow[b]{3}{*}{$\begin{array}{l}\text { PESF Annual } \\
\text { Energy Rating (MWh) }\end{array}$} \\
\hline & $\mathbf{M}$ & HOLYOKE, CITY & OF & & \\
\hline & PESF & $\mathrm{PESF} * \mathrm{KW}$ & $\begin{array}{l}\text { Annual } \\
\text { Rating }\end{array}$ & $\begin{array}{l}\text { Energy } \\
\text { (MWh) }\end{array}$ & \\
\hline 790.00 & 0.50 & 395.00 & \multicolumn{2}{|c|}{2009.00} & 1004.50 \\
\hline $\begin{array}{l}\text { Unit } \\
\text { Type }\end{array}$ & & $\begin{array}{l}\text { Project } \\
\text { Status }\end{array}$ & Sam & $\begin{array}{l}\text { Latitude } \\
\text { Longitude }\end{array}$ & $\begin{array}{r}4212.00 \\
7236.00\end{array}$ \\
\hline$c$ & & NO & wo & & \\
\hline
\end{tabular}

Factor

Wild/Scenic Protection

Wild/Scenic Tributary or Upstream/ Downstream wild/Scenic Location Cultural value Fish Presence Value Geologic Value Historic Value Other Value Recreation value Scenic Value
Exist Prob

Factor

Exist Prob

.

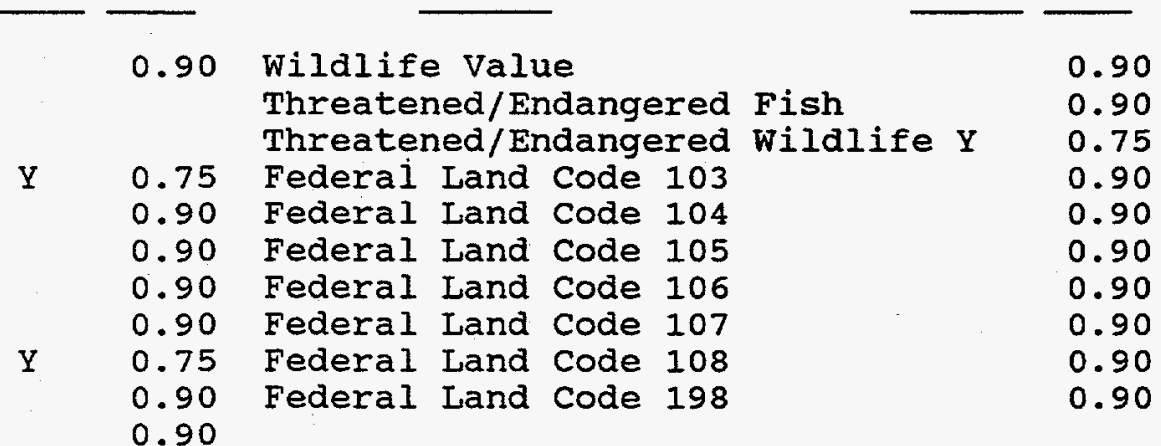


R E S O U R C E D A T A B A S E I I S T I N G

DATE: $06 / 27 / 95$

PAGE NO: 79

FERC

Number

Plant Name

stream

State

Name

11001 NASHUA

NASHUA $R$

MA

county Name

MIDDLESEX
River Basin

MERRIMACK RIVER BASIN

\section{Class Owner Name}

R A.L.L.NATURAL RESOURCES CO

$\begin{array}{rrrrr}\begin{array}{l}\text { Name Plate } \\ \text { Rating (KW) }\end{array} & \text { PESF } & \text { PESF*KW } & \begin{array}{l}\text { Annual Energy } \\ \text { Rating (MWh) }\end{array} & \begin{array}{c}\text { PESF Annual } \\ \text { Energy Rating (MWh) }\end{array} \\ 500.00 & 0.75 & 375.00 & 2500.00 & 1875\end{array}$

\begin{tabular}{|c|c|c|c|c|}
\hline $\begin{array}{l}\text { Unit } \\
\text { Type }\end{array}$ & $\begin{array}{l}\text { Plant } \\
\text { Type }\end{array}$ & $\begin{array}{l}\text { Project } \\
\text { status }\end{array}$ & $\begin{array}{l}\text { Dam } \\
\text { Status }\end{array}$ & $\begin{array}{l}\text { Latitude } \\
\text { Longitude }\end{array}$ \\
\hline
\end{tabular}

$\begin{array}{llll}\text { C ROR } & \text { PO }\end{array}$

\section{Factor}

wild/Scenic Protection

wild/Scenic Tributary or Upstream/Downstream wild/Scenic Location Cultural value Fish Presence Value Geologic Value Historic Value other Value Recreation Value Scenic Value
Exist Prob

0.90

Y
Factor

Exist Prob

$\begin{array}{lll}0.90 & \text { Wildife Value } & 0.90 \\ & \text { Threatened/Endangered Fish } & 0.90 \\ \text { Yhreatened/Endangered Wildlife } & 0.90 \\ 0.75 & \text { Federal Land Code 103 } & 0.90 \\ 0.90 & \text { Federal Land Code 104 } & 0.90 \\ 0.90 & \text { Federal Land Code 105. } & 0.90 \\ 0.90 & \text { Federal Land Code 106 } & 0.90 \\ 0.90 & \text { Federal Land Code 107 } & 0.90 \\ 0.90 & \text { Federal Land Code 108 } & 0.90 \\ 0.90 & \text { Federal Land Code 198 } & 0.90\end{array}$

0.90

0.90

0.90

0.90

0.90

0.90

0.90

0.90 
FERC

Number

11002
Plant Name

ARDEN MILL

county Name

WORCESTER

Class

Owner Name

$\mathbf{R}$

A.L.L.NATURAL RESOURCES

Name Plate Rating (KW)

PESF

$\mathrm{PESF} * \mathrm{KW}$

Annual Energy Rating (MWh)

200.00

$0.75 \quad 150.00$

Plant

Type

Project

status

PO

1000.00

Latitude

Longitude

4232.00

status

WO

$\begin{array}{llll}\text { C ROR } & \text { PO }\end{array}$

750
State

Name

MA
Factor

wild/Scenic Protection

wild/Scenic Tributary or Upstream/Downstream wild/Scenic Location Cultural value Fish Presence Value

Geologic Value Historic Value other Value Recreation Value Scenic Value
Exist Prob

0.90

Wildife Value Threatened/Endangered Fish Threatened/Endangered Wildlife

0.90 Federal Land Code 103

0.90 Federal Land code 104

$\begin{array}{lll}Y & 0.75 & \text { Federal Land Code } 105 \\ Y & 0.90 & \text { Federal Land Code } 106\end{array}$

0.90 Federal Land code 107

0.90 Federal Land code 108

0.90 Federal Land Code 198
PESF Annual Energy Rating (MWh)

0.90

Exist Prob

0.90

0.90

0.90

0.90

0.90

0.90

0.90

0.90

0.90

0.90 


\section{R E S O U R C E D A T A B A S E I I S T I N G}

DATE : $06 / 27 / 95$

PAGE NO: 81

FERC

Number

Plant Name

Stream

State

11058

FITCHBURG 4

N NASHUA R

Name

county Name

WORCESTER

River Basin

MERRIMACK RIVER BASIN

class Owner Name

R A.L.L.NATURAL RESOURCES

Name Plate

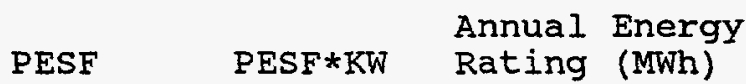

PESF Annual

Rating ( $\mathrm{KW}$ )

$0.75 \quad 37.50$

260.00

195

\begin{tabular}{|c|c|c|c|c|c|}
\hline $\begin{array}{l}\text { Unit } \\
\text { Type }\end{array}$ & $\begin{array}{l}\text { Plant } \\
\text { Type }\end{array}$ & $\begin{array}{l}\text { Project } \\
\text { status }\end{array}$ & $\begin{array}{l}\text { Dam } \\
\text { Status }\end{array}$ & $\begin{array}{l}\text { Latitude } \\
\text { Longitude }\end{array}$ & $\begin{array}{l}4230.00 \\
7147.00\end{array}$ \\
\hline C & ROR & NA & wo & & \\
\hline
\end{tabular}

Factor

wild/Scenic Protection

wild/Scenic Tributary or Upstream/Downstream wild/Scenic Location Cultural value

Fish Presence Value

Geologic Value

Historic Value

other Value

Recreation Value

scenic Value
Exist Prob
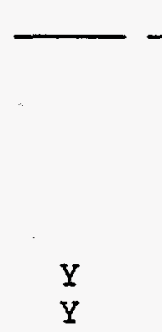

Factor

$\begin{array}{ll}0.90 & \text { Wildlife Value } \\ & \text { Threatened/Endangered Fish } \\ & \text { Threatened/Endangered Wildlife } \\ 0.90 & \text { Federal Land Code } 103 \\ 0.90 & \text { Federal Land Code } 104 \\ 0.75 & \text { Federal Land Code } 105 \\ 0.90 & \text { Federal Land Code } 106 \\ 0.90 & \text { Federal Land Code } 107 \\ 0.90 & \text { Federal Land Code } 108 \\ 0.90 & \text { Federal Land Code } 198 \\ 0.90 & \end{array}$

MA Energy Rating (MWh)

Exist Prob

0.90

0.90

0.90

0.90

0.90

0.90

0.90

0.90

0.90

0.90 
DATE: $06 / 27 / 95$

PAGE NO: 82

FERC

Number

11069
Plant Name

RISINGDALE

County Name

BERKSHIRE
River Basin

HOUSATONIC RIVER BASIN
State

Name

MA
HOUSATONIC $\mathrm{R}$

stream

Class Owner Name

R RISINGDALE HYDROLELECTRIC CO

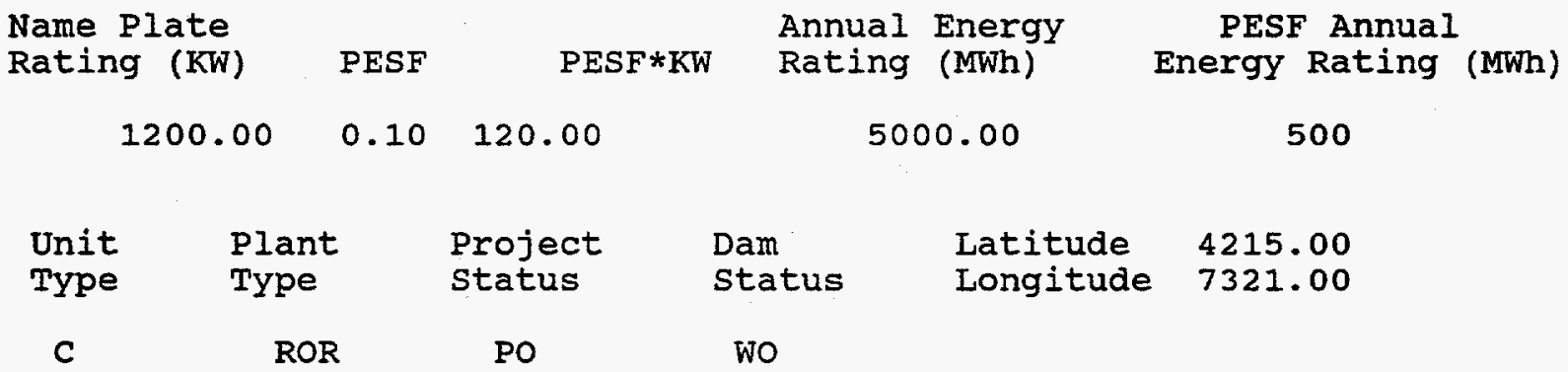

Factor

wild/Scenic Protection wild/scenic Tributary or Upstream/Downstream wild/Scenic Location Cultural value Fish Presence Value Geologic Value Historic Value other value Recreation Value Scenic Value
Exist Prob

0.90

$\mathbf{Y}$

0.75

0.90

0.75

$\mathrm{Y}$

$Y$
Factor

Exist Prob

\subsection{0}

0.90

0.90

0.90

0.90
0.90

0.90

0.90 Threatened/Endangered Wildlife Federal Land Code 103 Federal Land Code 104

0.10 Federal Land Code 105 Federal Land code 106 Federal Land Code 107 Federal Land Code 108 Federal Land Code 198

0.90

0.90

0.90

0.90

0.90

0.90 

R E S O U R C E
D A T A B A $\mathbf{S} \mathbf{E}$
I I S T I N G

DATE: $06 / 27 / 95$

PAGE NO: 83

FERC

Number

Plant Name

stream

state

11102 MIDDLESEX

CONCORD R

Name

\section{2}

County Name

River Basin

MIDDIESEX

MERRIMACK RIVER BASIN

MA

\section{Class Owner Name}

$\mathrm{R}$ OLSON ELECTRIC DEVELOP CO

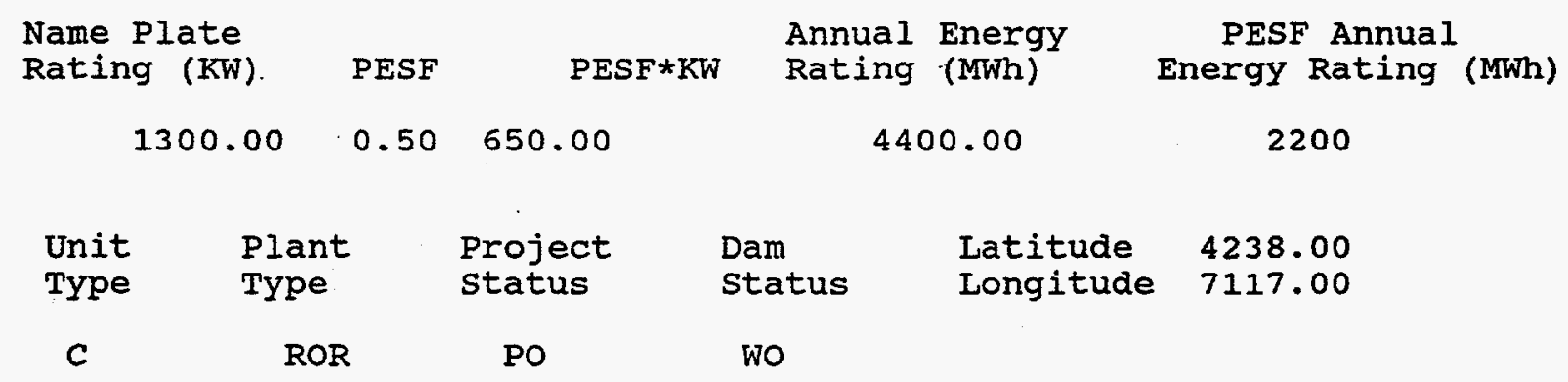

Factor

wild/Scenic Protection

wild/Scenic Tributary or

Upstream/Downstream wild/Scenic Location Cultural value

Fish Presence Value

Geologic Value

Historic Value

Other Value

Recreation Value

Scenic Value

\section{Exist Prob}

$\mathrm{Y}$

0.50

Wildife Value

Threatened/Endangered Fish

Threatened/Endangered Wildife

Y 0.75 Federal Iand code 103

0.90 Federal Land code 104

0.90 Federal Land code 105

0.90 Federal Land Code 106

$\mathrm{Y} \quad 0.75$

0.90

$Y \quad 0.75$

Federal Land code 198
Exist Prob

0.90

0.90

0.90

0.90

0.90

0.90

0.90

0.90

0.90

0.90

0.90 
DATE: $06 / 27 / 95$

PAGE NO: 84

FERC

Number

Plant Name

11111 CROCKER BURBANK MILL 6

county Name

WORCESTER

River Basin

MERRIMACK RIVER BASIN
State

Name

MA

Class Owner Name

R A.I.L.NATURAI RESOURCES INC

Name Plate

Annual Energy

Rating (KW)

$\mathrm{PESF} * \mathrm{KW}$

Rating (MWh)

PESF Annual

100.00

0.7575 .00

570.00

$427 \cdot 50$

$\begin{array}{ccclll}\text { Unit } & \text { Plant } & \text { Project } & \text { Dam } & \text { Latitude } & 4237.00 \\ \text { Type } & \text { Type } & \text { Status } & \text { Status } & \text { Longitude } & 7150.00 \\ \text { C } & \text { ROR } & \text { PO } & \text { WO } & \end{array}$

Factor

Exist Prob

Factor

Exist Prob

wild/Scenic Protection

wild/Scenic Tributary or Upstream/Downstream

wild/scenic Location

Cultural value

Fish Presence Value

Geologic Value

Historic Value

other Value

Recreation Value

Scenic Value

exist

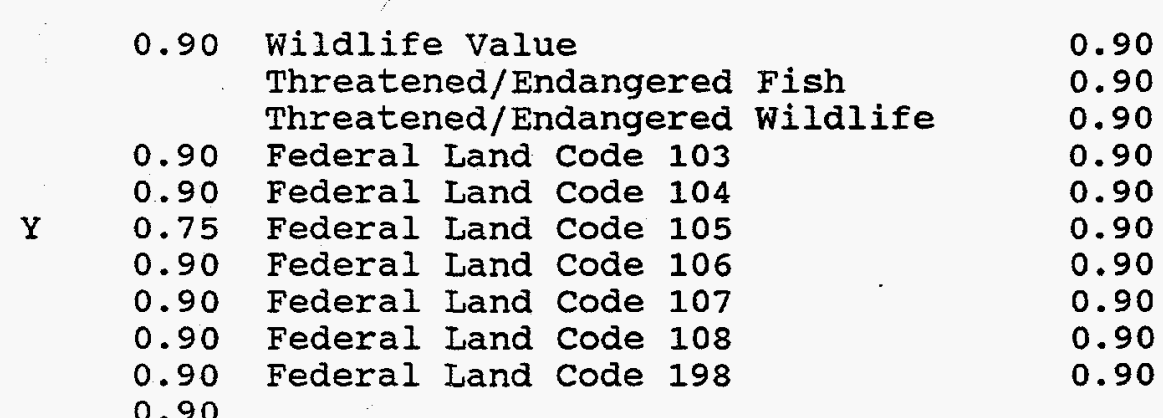



R E S O U R C E
D A T A B A S E
I I S T N G

DATE: $06 / 27 / 95$

PAGE NO: 85

FERC

Number

11166
Plant Name

SNOWS MILL POND

county Name

WORCESTER stream

WHITMAN R, NASHUA $R$

River Basin

MERRIMACK RIVER BASIN
State

Name

MA

\section{Class Owner Name}

$R$ OLESIN, ANDREW S

Name Plate

Rating (KW)

Annual Energy Rating (MWh)

PESF*KW

$0.90 \quad 315.00$

350.00

\begin{abstract}
Plant Type
\end{abstract}

ROR
Project status
PO

Exist Prob

Factor

wild/Scenic Protection Wild/Scenic Tributary or Upstream/Downstream wild/Scenic Location Cultural Value Fish Presence Value Geologic Value Historic Value other Value Recreation Value Scenic Value

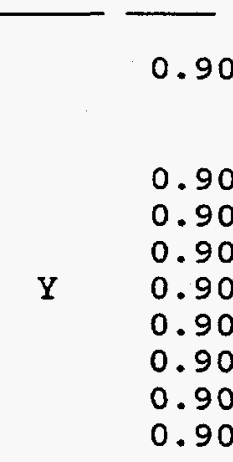

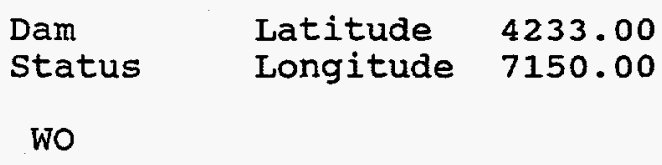

WO 
DATE: $06 / 27 / 95$

PAGE NO: 86

FERC

Number

11306
Plant Name

ECLIPSE DAM

county Name

BERKSHIRE state

Name

NBR HOOSIC RIVER

MA

River Basin

HUDSON RIVER BASIN

Class Owner Name

M NORTH ADAMS, CITY OF

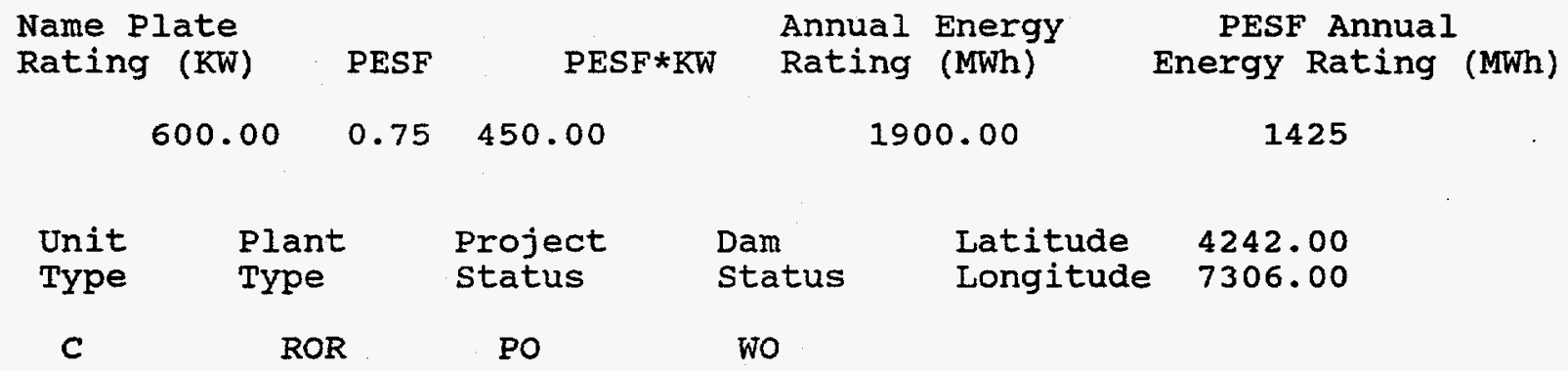

Factor

Exist Prob

Factor

Exist Prob

wild/Scenic Protection

Wild/Scenic Tributary or Upstream/Downstream

wild/Scenic Location Cultural value

Fish Presence Value

Geologic Value

Historic Value

other value

Recreation Value

Scenic Value

0.90 Wildlife Value Threatened/Endangered Fish Threatened/Endangered Wildiife

Y 0.75 Federal Land Code 103

0.90 Federal Land Code 104

0.90 Federal Land code 105

0.90 Federal Land Code 106

0.90 Federal Land Code 107

0.90 Federal Land Code 108

0.90 Federal Land Code 198

0.90
0.90

0.90

0.90

0.90

0.90

0.90

0.90

0.90

0.90

0.90 
RESOURCE DA TA B A S E I I S T I G

DATE: $06 / 27 / 95$

PAGE NO: 87

FERC

Number

11337
Plant Name

WHITNEY POND \#1

county Name

WORCESTER stream

MILLERS $\mathrm{R}$

River Basin

CONNECTICUT RIVER BASIN

\section{Class Owner Name}

R MORELAND ENERGY CO

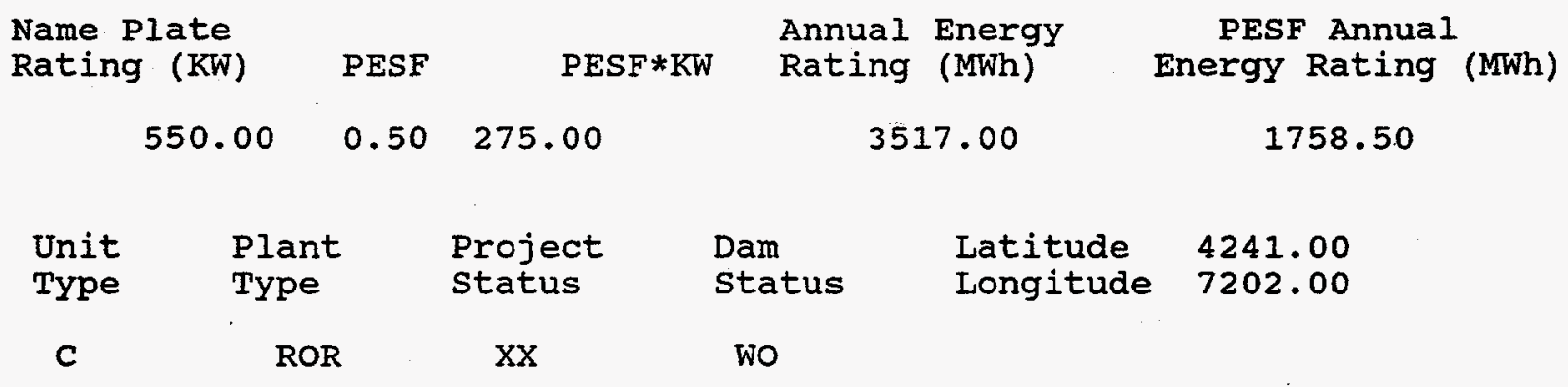

Factor

Wild/Scenic Protection

Wild/Scenic Tributary or Upstream/Downstream wild/Scenic Location Cultural value

Fish Presence Value Geologic Value Historic value other Value Recreation Value Scenic Value
Exist Prob

0.90 Wildlife Value Threatened/Endangered Fish Threatened/Endangered Wildlife

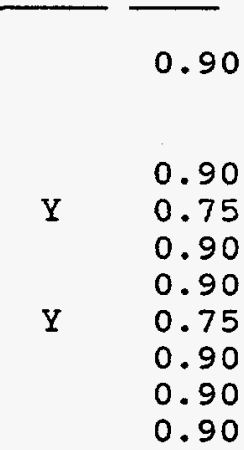

Exist Prob

Factor

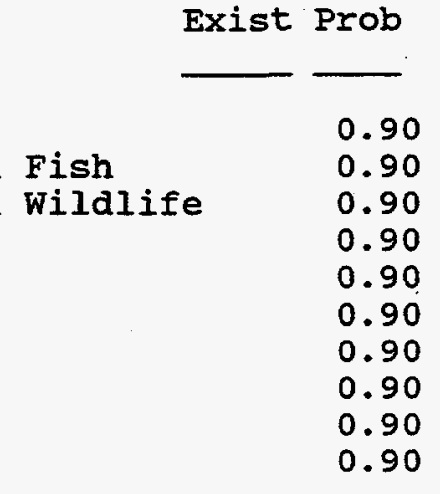
Federal Land Code 103 Federal Land Code 104 Federal Land code 105 Federal Land code 106 Federal Land Code 107 Federal Land Code 108 Federal Land code 198
State Name

MA 
FERC

Number

Plant Name
STONY BK, SUDBURY R

River Basin

MERRIMACK RIVER BASIN
State

Name

MA county Name

MIDDLESEX
PESF Annual Energy Rating (MWh)

$\begin{array}{rrrr}\begin{array}{l}\text { Name Plate } \\ \text { Rating (KW) }\end{array} & \text { PESF } & \text { PESF*KW } & \begin{array}{c}\text { Annual Energy } \\ \text { Rating (MWh) }\end{array} \\ 265.00 & 0.50 & 132.50 & 1470.00\end{array}$

Unit plant

Type Type

C

ROR

\section{Dam status}

Wo
Factor

wild/scenic Protection wild/Scenic Tributary or Upstream/Downstream wild/Scenic Location Cultural value Fish Presence Value Geologic Value Historic Value Other Value Recreation Value Scenic Value
Exist Prob

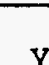

$\mathrm{Y}$

0.50

$\mathrm{Y}$

0.75

0.90

$\mathrm{Y} \quad 0.75$

0.90

0.90

0.90

Y $\quad 0.75$

0.90
Factor

Latitude Longitude
735

4219.00

7130.00 
RESOURCE D A T A B A S E I I S T I N G

DATE: $06 / 27 / 95$

PAGE NO: 89

FERC

Number

$\mathrm{MAOO2}$
Plant Name

OLD GUARD LOCKS

county Name

MIDDLESEX

\section{Stream}

MERRIMACK CNL (MERR R)

River Basin

MERRIMACK RIVER BASIN
State

Name

MA

\section{Class Owner Name}

NHPS/COE

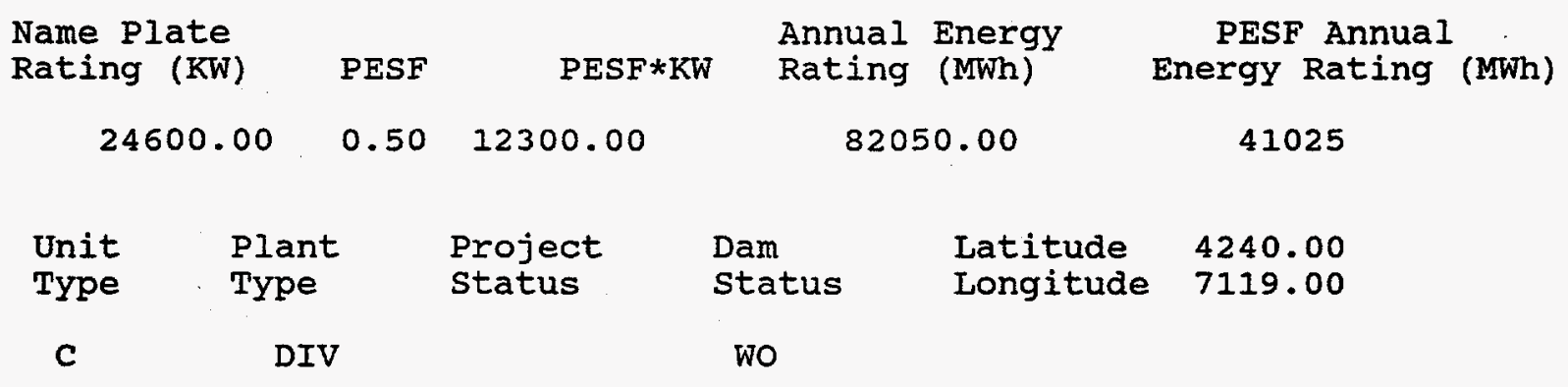

Factor

wild/Scenic Protection wild/Scenic Tributary or Upstream/Downstream wild/Scenic Location Cultural Value Fish Presence Value Geologic Value Historic Value other Value Recreation value Scenic Value
Exist Prob

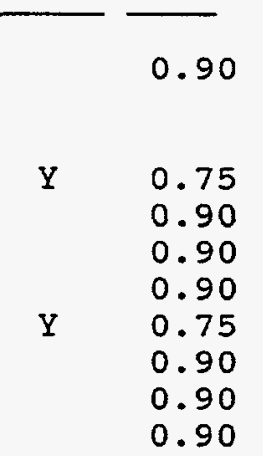

Factor

Exist Prob

Wildlife Value

Threatened/Endangered Fish Threatened/Endangered Wildlife

Federal Land code 103

Federal Land Code 104

Federal Land code 105

Federal Land Code 106

Federal Land code 107

Federal Land code 108

Federal Land Code 198 
DATE: $06 / 27 / 95$

PAGE NO: 90

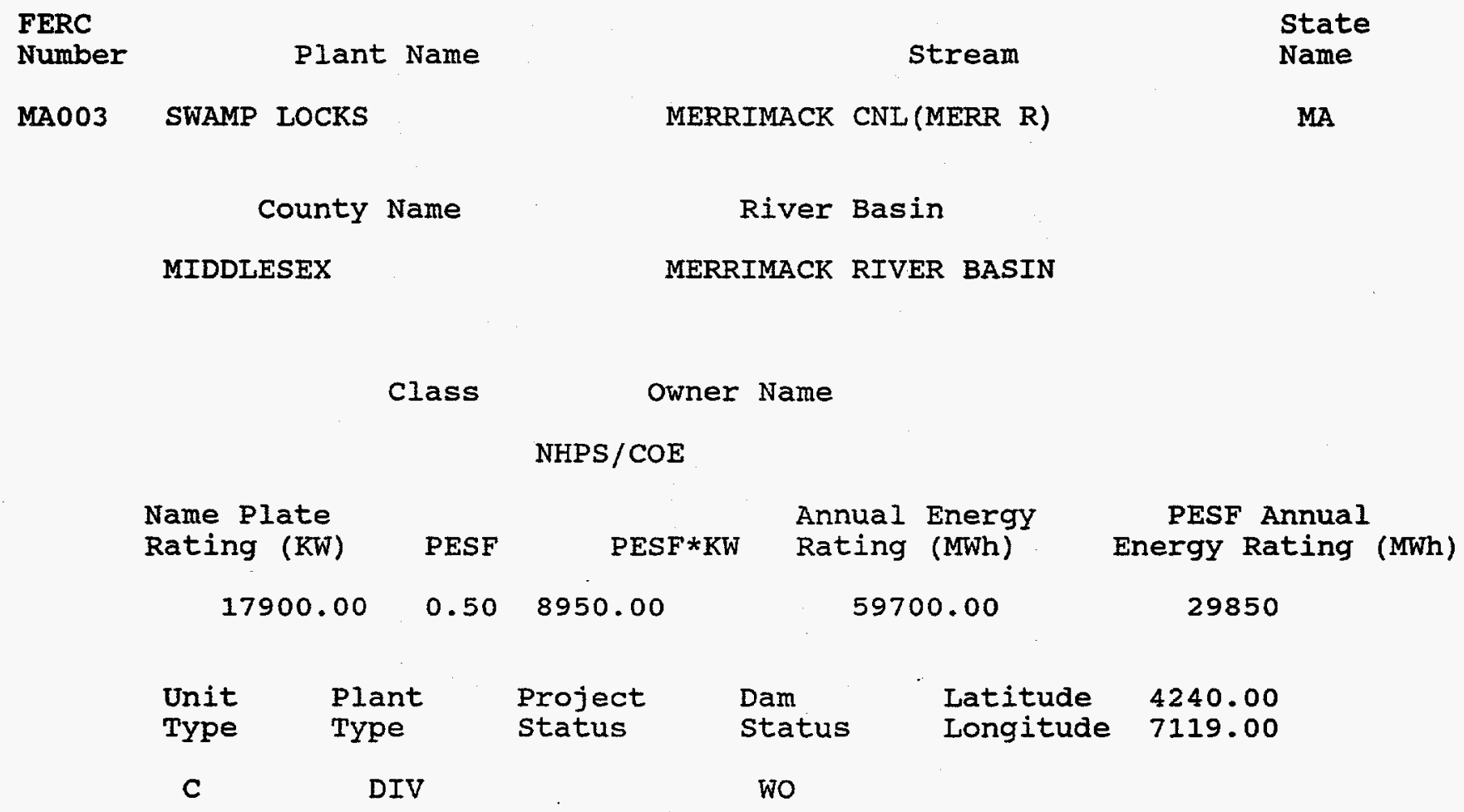

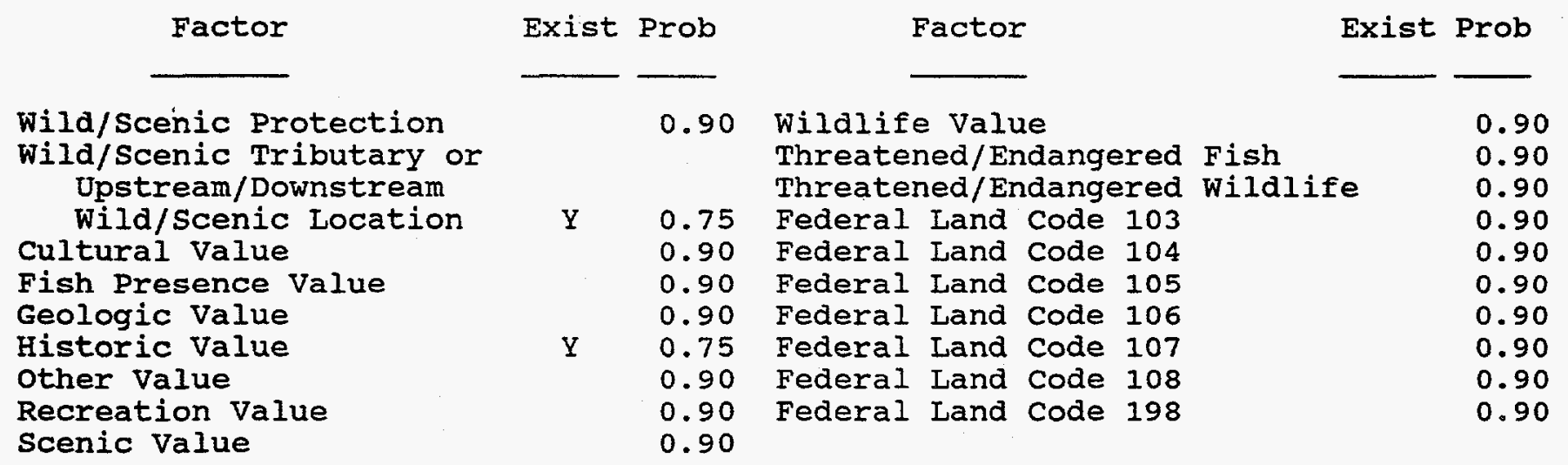


R E S O U R E D A T A B A S E L I S T I N G

DATE: $06 / 27 / 95$

PAGE NO: 91

FERC

Number

Plant Name

Stream

state

Name

MA004 PAWTUCKET (4)

FOUR CANALS (MERR R)

MA

county Name

River Basin

MIDDLESEX

MERRIMACK RIVER BASIN

\section{Class Owner Name}

I BOOTT MILLS ET AL

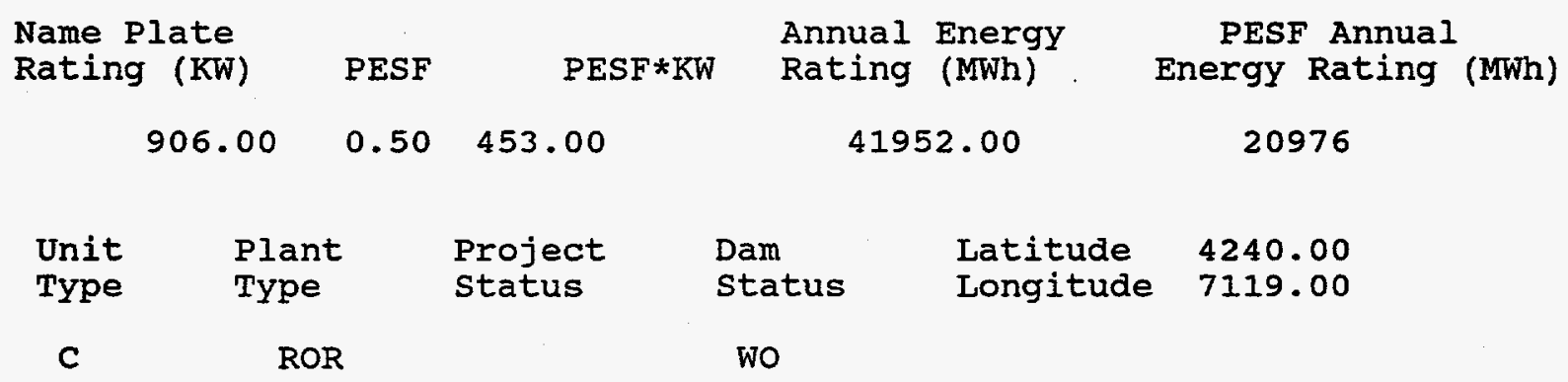

Factor

wild/Scenic Protection

wild/Scenic Tributary or Upstream/Downstream wild/Scenic Location Cultural Value

Fish Presence value

Geologic Value

Historic Value

other Value

Recreation Value

Scenic Value
Exist Prob

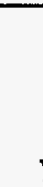

Y

Threatened/Endangered Fish

Threatened/Endangered Wildlife

Exist Prob

Factor

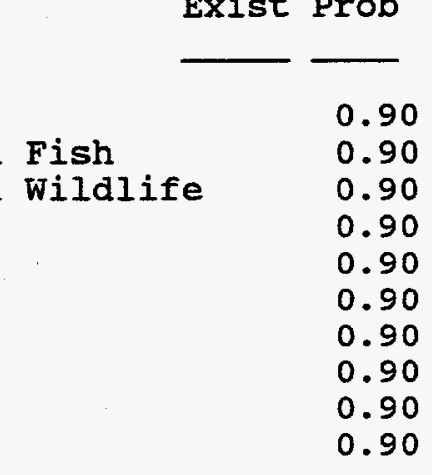


FERC

Number

Plant Name

MA005 NORTH CANAL LOCKS

County Name

MIDDLESEX
State

Name

MERRIMACK CNL (MERR R)

MA
River Basin

MERRIMACK RIVER BASIN
Class Owner Name

I JACKSON PROPS

$\begin{array}{lll}\text { Name Plate } & & \text { Annual Energy } \\ \text { Rating (KW) } & \text { PESF } & \text { PESF*KW } \\ \text { Rating (MWh) }\end{array}$

$33600.00 \quad 0.50 \quad 16800.00 \quad 112000.00$

Dam Latitude 4240.00

Status Longitude 7119.00

Wo
Factor

Wild/Scenic Protection

wild/Scenic Tributary or Upstream/Downstream wild/Scenic Location cultural value

Fish Presence Value

Geologic Value

Historic Value

other value

Recreation Value

Scenic Value
Exist Prob

0.90

wildlife Value Threatened/Endangered Fish Threatened/Endangered Wildlife

Exist Prob

Y 0.75 Federal Land Code 103

0.90 Federal Land Code 104

0.90 Federal Land Code 105

0.90 Federal Land code 106

$\mathrm{Y} \quad 0.75$ Federal Land code 107

0.90 Federal Land Code 108

0.90 Federal Land Code 198

0.90
PESF Annual Energy Rating (MWh)

56000 
RESOU R E

DATE : $06 / 27 / 95$

FERC

Number

Plant Name

MA006

PEPPERELL

County Name

FRANKLIN
D A T A B A S E

L I S T I G

PAGE NO: 93

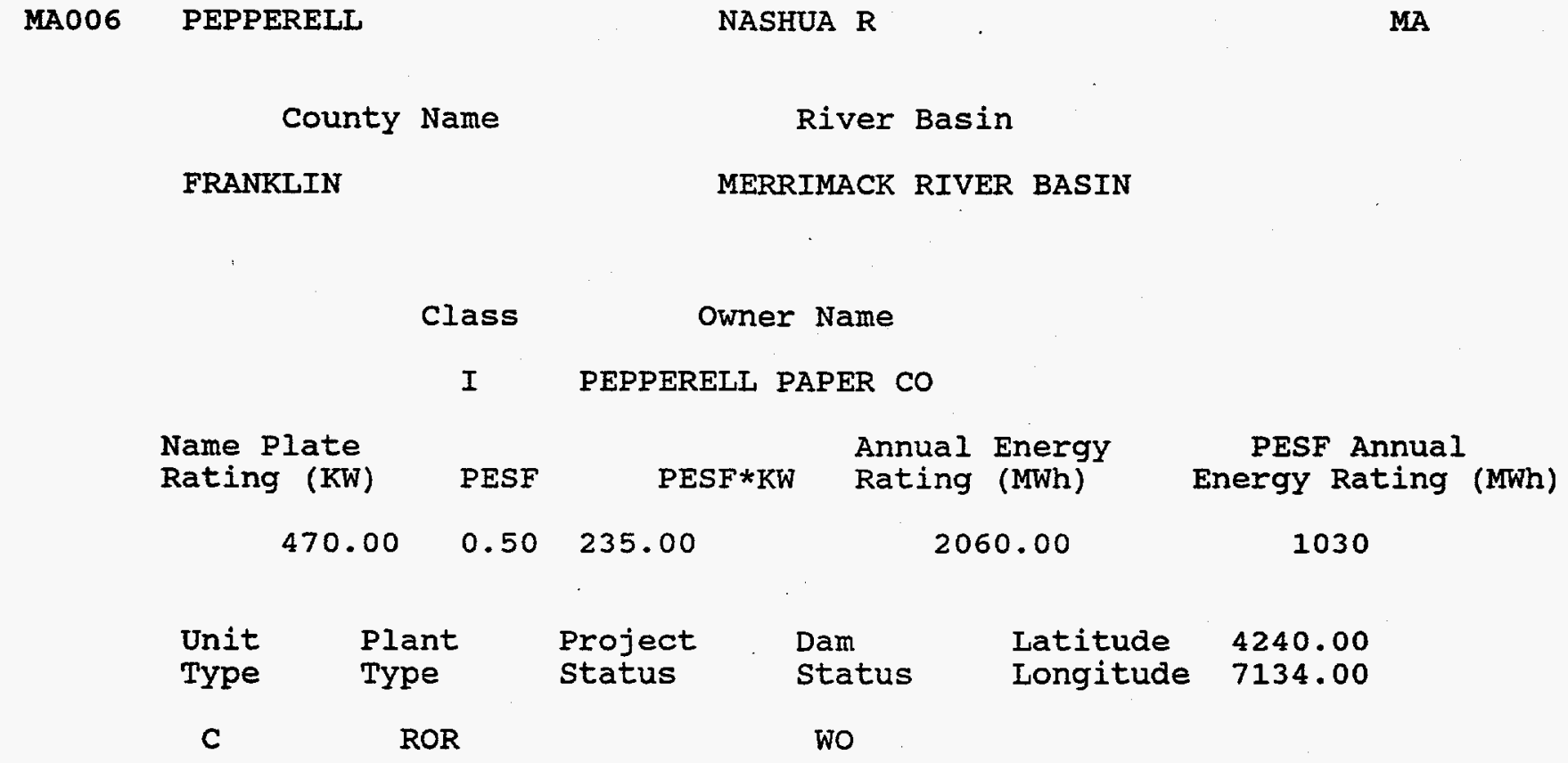

Exist Prob

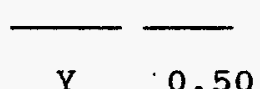

$\mathrm{Y} \quad 0.50$

wild/Scenic Protection

wild/Scenic Tributary or Upstream/Downstream wild/Scenic Location Cultural value Fish Presence Value Geologic Value Historic Value Other Value Recreation Value Scenic Value stream

State Name

MA 
DATE: $06 / 27 / 95$

PAGE NO: 94

FERC

Number

$\mathrm{MAO07}$
Plant Name

IANCASTER MILI POND

county Name

WORCESTER stream

NASHUA $R$

River Basin

MERRIMACK RIVER BASIN state

Name

MA

\section{Class Owner Name}

I IANCASTER MILLS

$\begin{array}{rrrr}\begin{array}{l}\text { Name Plate } \\ \text { Rating (KW) }\end{array} & \text { PESF } & \text { PESF*KW } & \begin{array}{c}\text { Annual Energy } \\ \text { Rating (MWh) }\end{array} \\ 360.00 & 0.25 & 90.00 & 1995.00\end{array}$

$\begin{array}{lllll}\text { Unit } & \text { Plant } & \text { Project } & \text { Dam } & \text { Latitude } \\ \text { Type } & \text { Type } & \text { Status } & \text { Status } & \text { Longitude } 7141.00\end{array}$

ROR

U

Factor

Wild/Scenic Protection

wild/scenic Tributary or Upstream/Downstream wild/Scenic Location Cultural value

Fish Presence Value

Geologic Value

Historic Value

other value

Recreation Value

Scenic Value
Exist Prob

0.90

wildife Value Threatened/Endangered Fish Threatened/Endangered Wildlife

0.50 Federal Land code 103

0.90 Federal Land Code 104

$\begin{array}{lll}Y & 0.25 & \text { Federal Land Code } 105 \\ Y & 0.50 & \text { Federal Land code } 106\end{array}$

$Y \quad 0.50 \quad$ Federal Land code 106

$\mathrm{Y} \quad 0.50$ Federal Land code 108

0.90 Federal Land code 198

0.90
0.90
PESF Annual Energy Rating (MWh)

498.75 

R E S O U R C E
D A T A B A S E
I I S T I N G

DATE: $06 / 27 / 95$

PAGE NO: 95

FERC

Number

Plant Name

stream

State

MA008 WACHUSETT

S BR NASHUA R

Name

MAOOS WACHUSETT

county Name

River Basin

WORCESTER

MERRIMACK RIVER BASIN

$\begin{array}{cr}\text { Class } & \text { Owner Name } \\ \text { M } & \text { METRO DIST COMM }\end{array}$

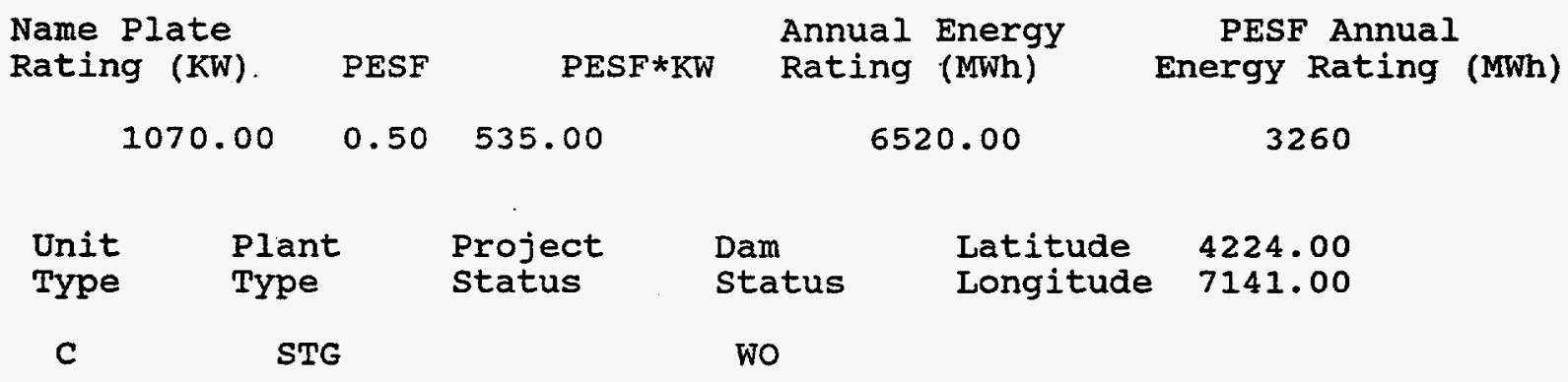

Factor

wild/Scenic Protection

wild/Scenic Tributary or Upstream/Downstream wild/scenic Location cultural value Fish Presence Value Geologic Value Historic Value Other Value Recreation Value Scenic Value

\section{Exist Prob}

Y

$$
0.50
$$

Wildlife Value

Threatened/Endangered Fish Threatened/Endangered Wildlife Exist Prob

$Y$

0.75

0.90

$\mathrm{Y} \quad 0.75$

$\mathrm{Y} \quad 0.90$

0.90

$\mathrm{Y} \quad 0.75$

0.90

0.90
Federal Land Code 103

Federal Land code 104

Federal Land Code 105

Federal Land Code 106

Federal Land Code 108

Federal Land Code 198
Federal Land Code 107
MA 
FERC

Number

MA009
Plant Name

MACTAGGARTS POND

County Name

WORCESTER

Class

Owner Name

I

Name Plate Rating ( $\mathrm{KW}$ )

250.00
PESF

$0.75 \quad 187.50$

Plant

Type

Project status

C

ROR
Annual Energy Rating (MWh)

1060.00
State Name

MA
PHILLIPS BK, $N$ NASHUA $R$

River Basin

MERRIMACK RIVER BASIN

\begin{tabular}{|c|c|c|c|c|c|}
\hline $\begin{array}{l}\text { Unit } \\
\text { Type }\end{array}$ & $\begin{array}{l}\text { Plant } \\
\text { Type }\end{array}$ & $\begin{array}{l}\text { Project } \\
\text { status }\end{array}$ & $\begin{array}{l}\text { Dam } \\
\text { status }\end{array}$ & $\begin{array}{l}\text { Latitude } \\
\text { Longitude }\end{array}$ & $\begin{array}{l}4235.00 \\
7151.00\end{array}$ \\
\hline & ROR & & wo & & \\
\hline
\end{tabular}

Factor

Wild/Scenic Protection wild/Scenic Tributary or Upstream/Downstream wild/Scenic Location Cultural value Fish Presence Value Geologic Value Historic Value other value Recreation Value Scenic Value
Exist Prob

0.90

Wildife Value Threatened/Endangered Fish Threatened/Endangered Wildiife

0.90 Federal Land Code 103

0.90 Federal Land code 104

0.75 Federal Land Code 105

$Y$

$\mathrm{Y}$

Federal Land code 106

0.90 Federal Land Code 107

0.90 Federal Land code 108

0.90 Federal Land Code 198

0.90

PESF Annual Energy Rating (MWh)

795

Exist Prob

0.90

0.90

0.90

0.90

0.90

0.90

0.90

0.90

0.90

0.90 
RES O U R E D A T A B A S E I I S T I N G

DATE: $06 / 27 / 95$

PAGE NO: 97

FERC

Number

Plant Name

Stream

state

MA010 FITCHBURG

N NASHUA R

Name

MAO10

county Name

River Basin

WORCESTER

MERRIMACK RIVER BASIN

Class Owner Name

I JAMES RIVER MASSACHUSETTS

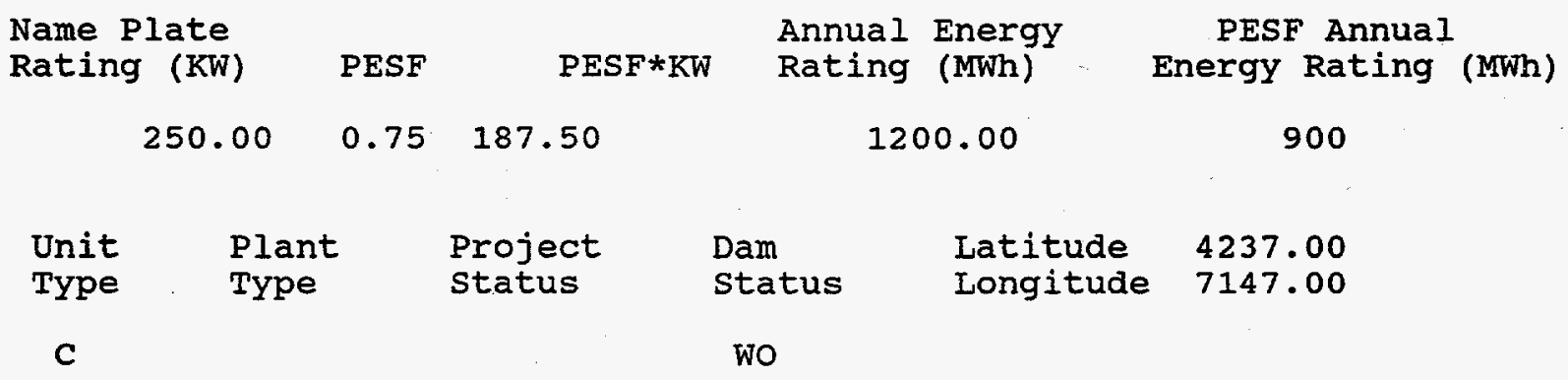

Factor

Exist Prob

wild/Scenic Protection

Wild/Scenic Tributary or Upstream/ Downstream wild/Scenic Location cultural value

Fish Presence Value

Geologic Value

Historic Value

other Value

Recreation Value

Scenic Value
Factor

Exist Prob

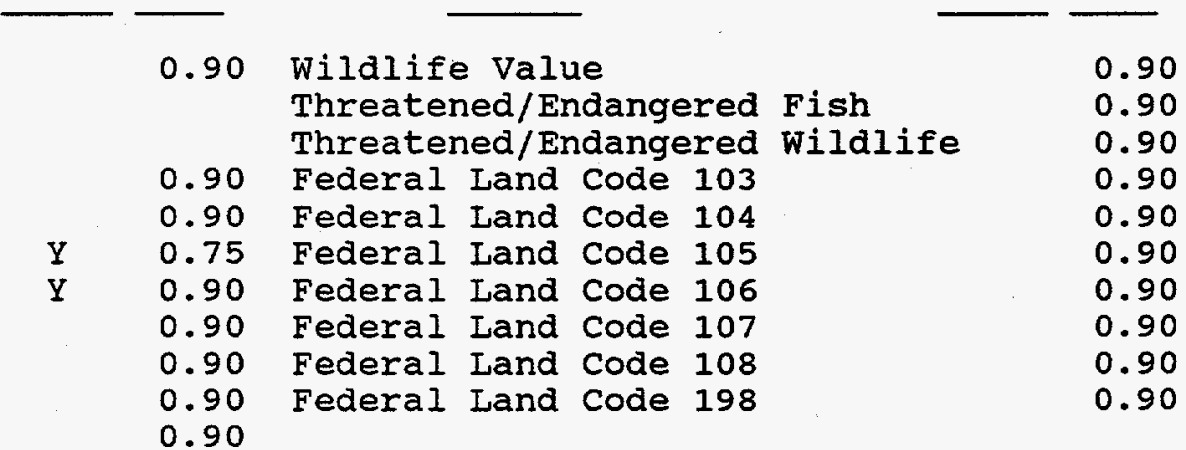


FERC

Number

Plant Name

MA011 WATERTOWN

county Name

MIDDLESEX

Class

Owner Name

M WATERTOWN, TOWN OF

Name Plate Rating (KW)

415.00

PESF

$0.25 \quad 103.75$

Plant

Type

Project

Unit
Type

ROR

Exist Prob

Factor

Latitude

Longitude

4222.00

status

U
Annual Energy

2100.00 state

Name

MA
Factor

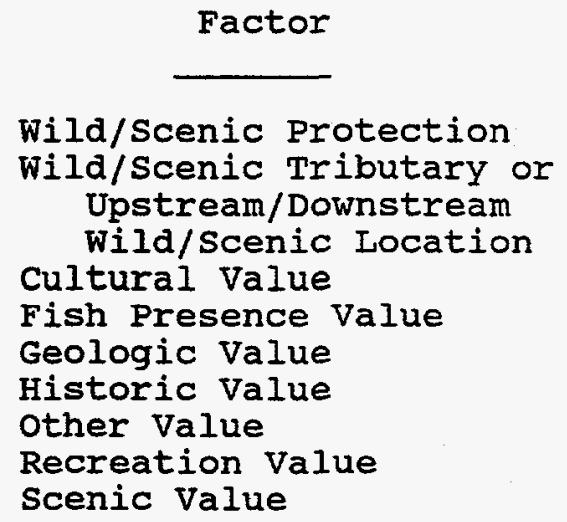

Scenic Value

wild/Scenic Protection

ld/Scenic Tributary or Upstream/Downstream Cultural value

Fish Presence Value

Geologic Value

Historic Value

other Value
PESF Annual Energy Rating (MWh)

525

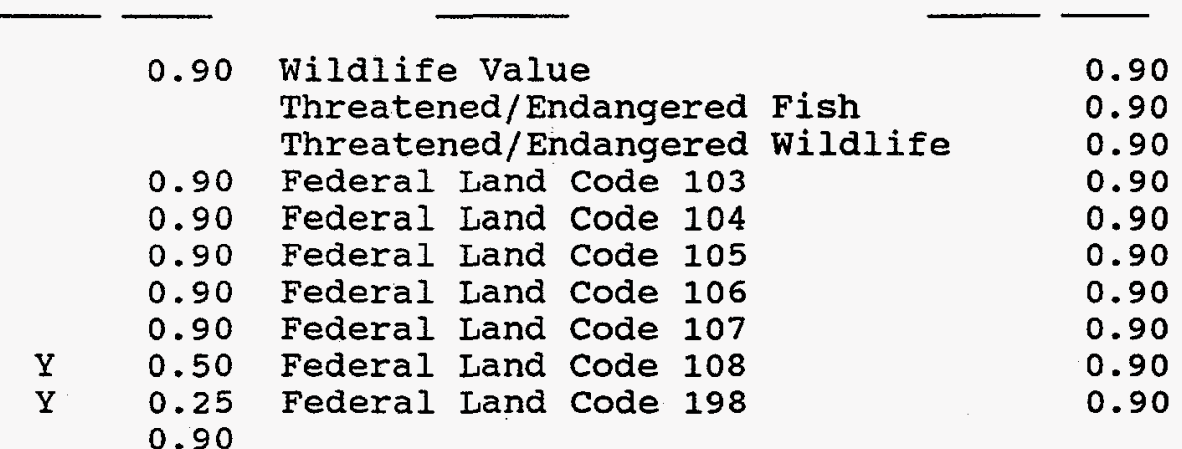


R E S U U C E D A T A B A S E I I S T I N G

DATE: $06 / 27 / 95$

PAGE NO: 99

FERC

Number

Plant Name

Stream

State

MA012 SOUTH WATUPPA POND

QUEQUECHAN R

Name

County Name

River Basin

BRISTOL

MINOR RIVER BASINS

Class Owner Name

M FALI RIVER, CITY OF

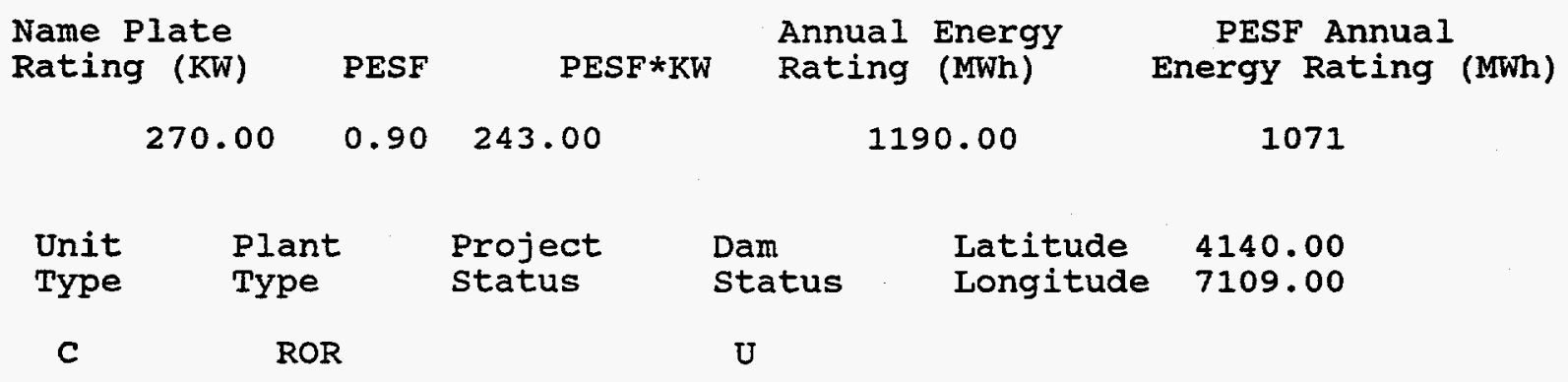

Factor

Exist Prob

Factor

Exist Prob

wild/scenic Protection

wild/Scenic Tributary or Upstream/Downstream wild/Scenic Location Cultural value

Fish Presence Value

Geologic Value

Historic Value

other value

Recreation Value

Scenic Value

$\begin{array}{ll}0.90 & \text { Wildife Value } \\ & \text { Threatened/Endangered Fish } \\ \text { Threatened/Endangered Wildlife } \\ 0.90 & \text { Federal Land Code } 103 \\ 0.90 & \text { Federal Land Code } 104 \\ 0.90 & \text { Federal Land Code } 105 \\ 0.90 & \text { Federal Land Code } 106 \\ 0.90 & \text { Federal Land Code } 107 \\ 0.90 & \text { Federal Land Code } 108 \\ 0.90 & \text { Federal Land Code } 198\end{array}$

\subsection{Wildife Value} Threatened/Endangered Fish Federal Land code 103

Federal Land code 104

Federal Land code 107

Federal Land Code 198
MA 
RE SOURCE D A T A B A S E L I S T I N G

DATE: $06 / 27 / 95$

PAGE NO: 100

FERC

Number

MA013
Plant Name

BLACKSTONE DAM

county Name

WORCESTER

Class
State

stream

BLACKSTONE R

MA
River Basin

MINOR RIVER BASINS

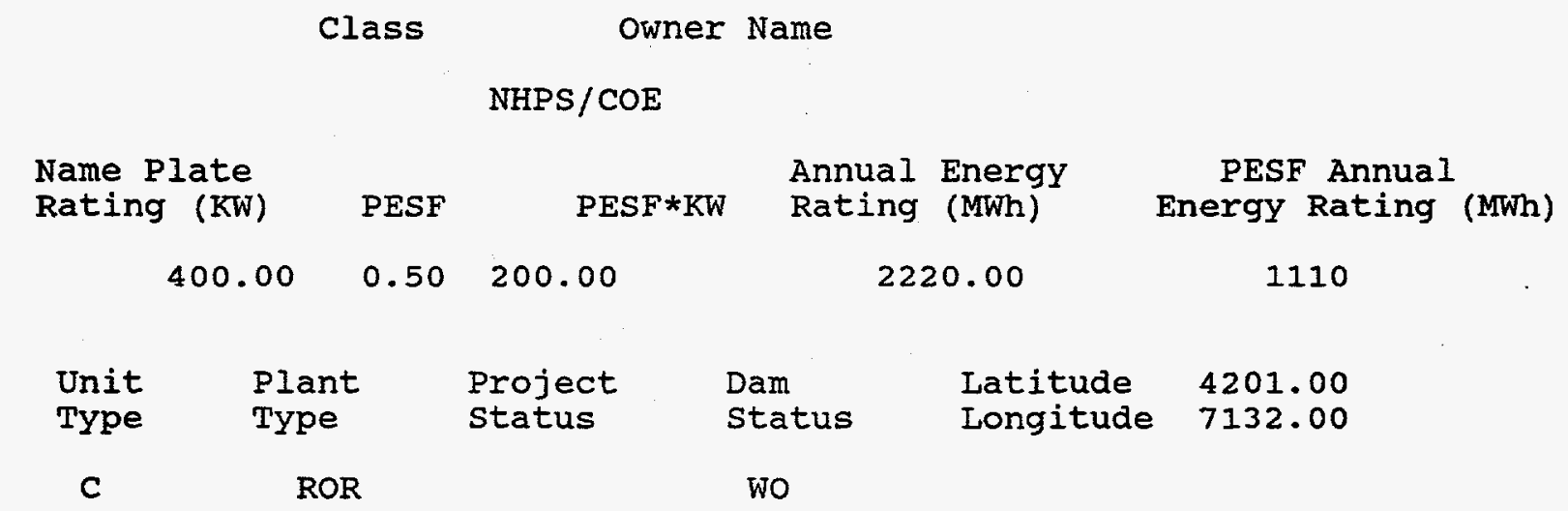

Factor

Exist Prob

Factor

Exist Prob

Wild/Scenic Protection

Wild/Scenic Tributary or

Upstream/Downstream

wild/Scenic Location

Cultural Value

Fish Presence value

Geologic Value

Historic Value

other value

Recreation value

Scenic Value

Exist Prob

0.90

0.90 Wildlife Value

0.90

Threatened/Endangered Fish

0.90

$\mathrm{Y}$

Threatened/Endangered Wildlife

0.90

0.75 Federal Land Code 103

0.90

0.90 Federal Land Code 104

0.90

0.90 Federal Land Code 105

0.90

Y $\quad 0.75$ Federal Land Code 107

0.90

0.90

Federal Land Code 108

0.90

0.90

Federal Land Code 198

0.90 
RES O URCE D A T A B A S E I I S T I N G

DATE: $06 / 27 / 95$

PAGE NO: 101

FERC

Number

Plant Name

stream

State

MA014 FISHERVILLE POND

QUINSIGAMOND R

Name

County Name

River Basin

WORCESTER

MINOR RIVER BASINS

Class Owner Name

I DURA LITE CO

Name Plate

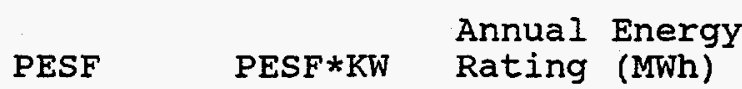

PESF Annual

Rating ( $\mathrm{KW}$

$0.25 \quad 106.25$

2100.00

Energy Rating (MWh)

425.00

525

$\begin{array}{cclll}\text { Unit } & \text { Plant } & \begin{array}{l}\text { Project } \\ \text { Status }\end{array} & \begin{array}{l}\text { Dam } \\ \text { Status }\end{array} & \begin{array}{l}\text { Latitude } \\ \text { Longitude }\end{array} \\ \text { Type } & \text { Ror } & \text { St1.00 } \\ \text { C } & \text { ROR } & & U & \end{array}$

Factor

wild/Scenic Protection

Wild/Scenic Tributary or Upstream/ Downstream wild/Scenic Location Cultural value

Fish Presence Value

Geologic Value

Historic Value

other Value

Recreation Value

Scenic Value
Exist Prob

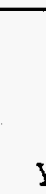

0.50
0.90
0.90

0.90

$\mathrm{Y} \quad 0.50$

0.90

0.90

0.90
Factor

Exist Prob

Wildlife value

Threatened/Endangered Fish

Threatened/Endangered wildife

Federal Land code 103

Federal Land Code 104

Federal Land Code 105

Federal Land code 106

Federal Land code 107

Federal Land code 108

Federal Land code 198
MA 
FERC

Number
Plant Name

MA015 QUINEBAUG $R$ POND

County Name

WORCESTER
QUINEBAUG $R$

River Basin

THAMES RIVER BASIN
State

Name

MA

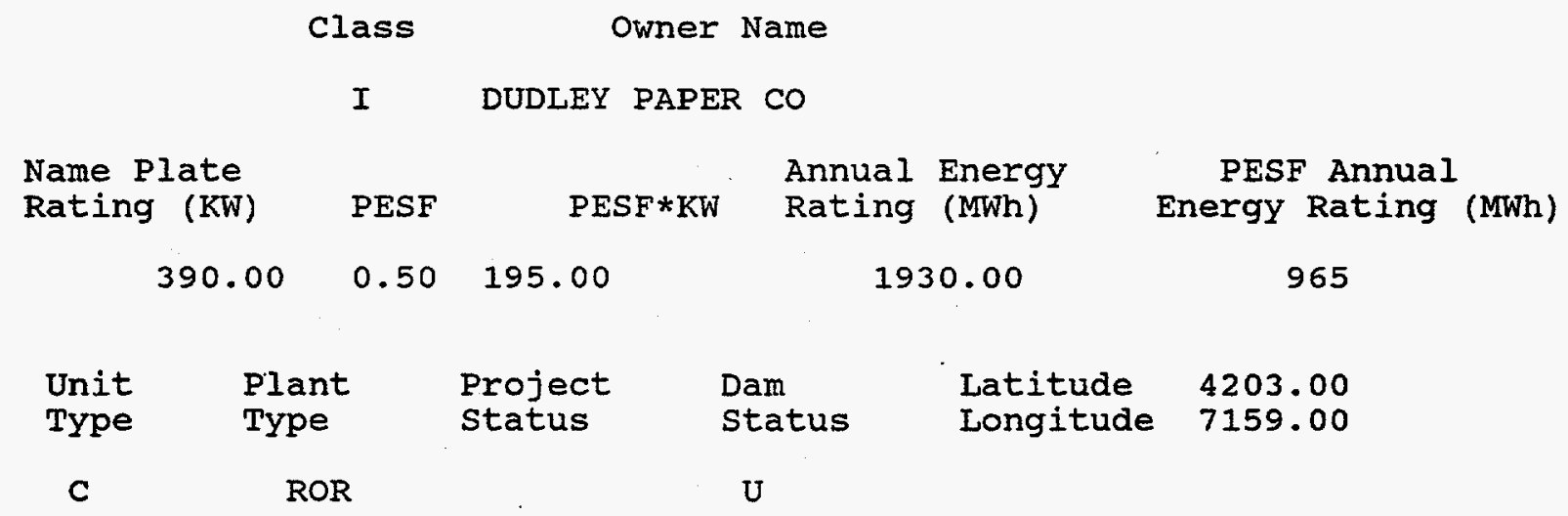

Factor

Wild/Scenic Protection wild/Scenic Tributary or Upstream/Downstream wila/Scenic Location Cultural value

Fish Presence Value

Geologic Value

Historic Value other Value

Recreation Value

Scenic Value
Exist Prob

0.90

Wildlife Value Threatened/Endangered Fish Threatened/Endangered Wildlife

$\mathrm{Y} \quad 0.50$ Federal Land code 103

0.90 Federal Land Code 104

0.90 Federal Land Code 105

0.90 Federal Land Code 106

0.90 Federal Land Code 107

0.90 Federal Land Code 108

$0.90^{\circ}$ Federal Land code 198

0.90
Exist Prob

0.90

0.90

0.90

0.90

0.90

0.90

0.90

0.90

0.90

0.90 
R E S O U R E D A T A B A S E L I S T I N G

DATE: $06 / 27 / 95$

PAGE NO: 103

FERC

Number

MA016
Plant Name

WEST SPRINGFIELD

County Name

HAMPDEN

\author{
State \\ Name \\ WESTFIEID $\mathrm{R}$ \\ MA \\ River Basin
}

CONNECTICUT RIVER BASIN

Class owner Name

I NEW YORK PREMOID CORP ET AL

\begin{tabular}{|c|c|c|c|c|c|c|c|}
\hline \multicolumn{2}{|c|}{$\begin{array}{l}\text { Name Plate } \\
\text { Rating (KW) }\end{array}$} & PESF & PESF*KW & \multicolumn{3}{|c|}{$\begin{array}{l}\text { Annual Energy } \\
\text { Rating (MWh) }\end{array}$} & $\begin{array}{l}\text { PESF Annual } \\
\text { Energy Rating (MWh) }\end{array}$ \\
\hline & 230.00 & 0.50 & 115.00 & \multicolumn{3}{|c|}{5100.00} & 2550 \\
\hline $\begin{array}{l}\text { Unit } \\
\text { Type }\end{array}$ & $\begin{array}{l}\text { Pla } \\
\text { TyF }\end{array}$ & & $\begin{array}{l}\text { Project } \\
\text { status }\end{array}$ & \multicolumn{2}{|l|}{$\begin{array}{l}\text { Dam } \\
\text { status }\end{array}$} & $\begin{array}{l}\text { Latitude } \\
\text { Longitude }\end{array}$ & $\begin{array}{l}4206.00 \\
7238.00\end{array}$ \\
\hline $\mathrm{C}$ & \multicolumn{2}{|c|}{ ROR } & \multicolumn{4}{|c|}{$U$} & \\
\hline
\end{tabular}

Factor

Exist Prob

Factor

Exist Prob

wild/Scenic Protection

wild/Scenic Tributary or Upstream/Downstream wild/Scenic Location cultural value Fish Presence Value Geologic Value Historic Value other Value

Recreation Value

scenic Value

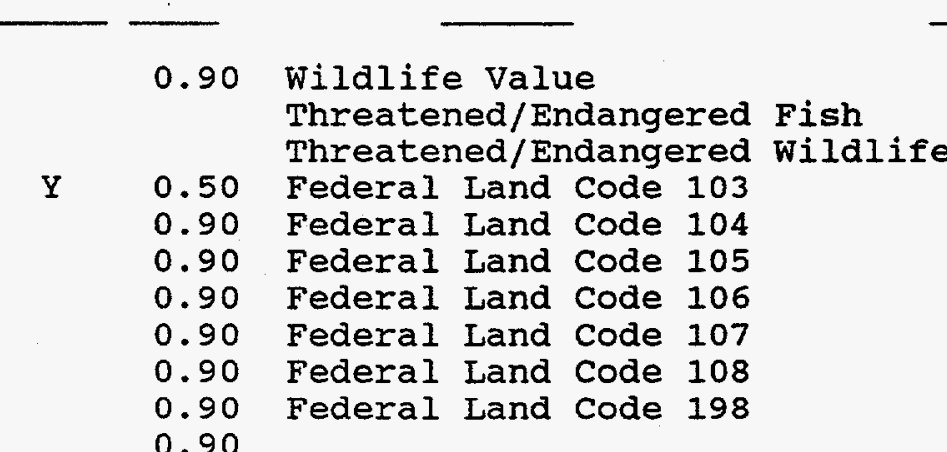


FERC

Number

Plant Name

MA017
GRANVILLE

County Name HAMPDEN
River Basin

CONNECTICUT RIVER BASIN
State

Name

MUNN BK, LITTLE R

MA

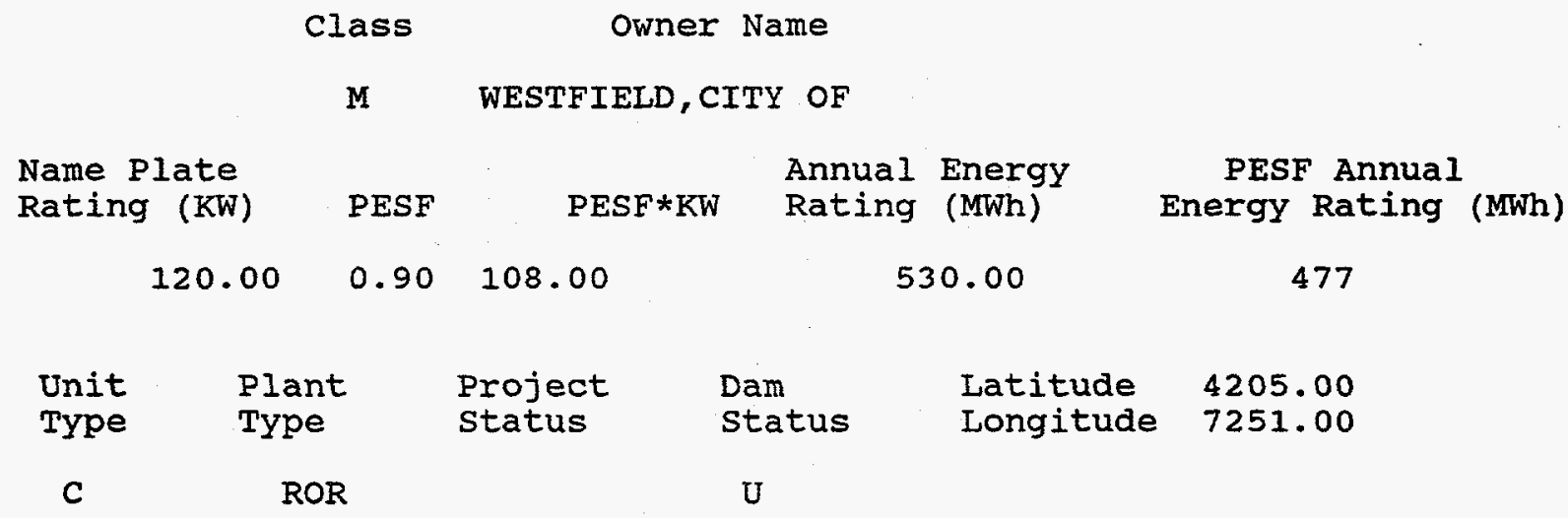

Factor

wild/Scenic Protection

Wild/Scenic Tributary or Upstream/Downstream wild/Scenic Location Cultural value Fish Presence Value

Geologic Value

Historic Value

other Value

Recreation Value

scenic Value
Exist Prob

Factor

Exist Prob

0.90

Wildlife Value

Threatened/Endangered Fish

Threatened/Endangered Wildife

0.90 Federal Land Code 103

0.90 Federal Land code 104

0.90 Federal Land code 105

0.90 Federal Land Code 106

0.90 Federal Land code 107

0.90 Federal Land Code 108

0.90

0.90
0.90

0.90

0.90

0.90

0.90

0.90

0.90

0.90

0.90

0.90 
RESOURCE D A T A B A S E L I S T I N G

DATE: $06 / 27 / 95$

PAGE NO: 105

FERC

Number

Plant Name

stream

State

Name

MA018 THE GORGE

LITTLE R

MA

county Name

HAMPDEN
River Basin

CONNECTICUT RIVER BASIN

\section{Class Owner Name \\ M SPRINGFIELD WATER WORKS}

Name Plate Rating (KW) PESF

Annual Energy Rating (MWh)

1900.00 490.00

$0.90 \quad 441.00$

$\begin{array}{lll}\text { Dam } & \text { Latitude } & 4207.00 \\ \text { Status } & \text { Longitude } & 7251.00\end{array}$

$\begin{array}{cc}\text { Unit } & \text { Plant } \\ \text { Type } & \text { Type } \\ \text { C } & \text { ROR }\end{array}$

Project status

Factor

wild/Scenic Protection

wild/Scenic Tributary or Upstream/Downstream wild/Scenic Location Cultural Value

Fish Presence Value

Geologic Value

Historic Value

Other Value

Recreation Value

Scenic Value
Exist prob

0.90 Wildlife Value

Threatened/Endangered Fish Threatened/Endangered Wildife

0.90 Federal Land code 103

0.90 Federal Land code 104

0.90 Federal Land code 105

0.90 Federal Land Code 106

0.90 Federal Land Code 107

0.90 Federal Land code 108

0.90 Federal Land code 198

0.90
1710

PESF Annual Energy Rating (MWh) 
$\dot{R} E S O U R C E \quad D A T A B A S E$ L I S T I N G

\author{
DATE : $06 / 27 / 95$
}

PAGE NO: 106

\title{
FERC
}

Number

Plant Name

stream

State

Name

\section{MA019 WORONCO (STRATMORE)}

County Name HAMPDEN

WESTFIELD $\mathbf{R}$

MA

River Basin

CONNECTICUT RIVER BASIN

Class Owner Name

I HAMMERMILL PAPER CO

Name Plate

$\begin{array}{ll}\text { PESF } & \begin{array}{l}\text { Annual Energy } \\ \text { PESF*KW }\end{array}\end{array}$

PESF Annual Rating ( $\mathrm{KW}$ )

0.75292 .50

6000.00

4500

390.00

0.75 .292 .50

$\begin{array}{lll}\text { Unit } & \text { Plant } & \text { Project } \\ \text { Type } & \text { Type } & \text { Status }\end{array}$

C

ROR

$\begin{array}{lll}\text { Dam } & \text { Latitude } & 4210.00 \\ \text { Status } & \text { Longitude } & 7249.00\end{array}$

WO

Factor

Exist Prob

wild/Scenic Protection

wild/Scenic Tributary or

Upstream/Downstream

wild/Scenic Location

Cultural Value

Fish Presence Value

Geologic Value

Historic Value

other Value

Recreation value

Scenic Value

Exist Prob
0.90

Wildife Value

Threatened/Endangered Fish

Threatened/Endangered WildIife

0.75 Federal Land code 103

0.90 Federal Land Code 104

0.90 Federal Land Code 105

0.90 Federal Land Code 106

0.90 Federal Land code 107

0.90 Federal Land code 108

0.90 Federal Land Code 198

0.90
Exist Prob

0.90

0.90

0.90

0.90

0.90

0.90

0.90

0.90

0.90

0.90 
DATE: $06 / 27 / 95$

FERC

Number

Plant Name

MAO20 RUSSELL

County Name

HAMPDEN

class

Owner Name

I WESTFIELD RIVER PAPER CO

Name Plate

Rating (KW)

PESF

PESF*KW

Annual Energy

Rating (MWh)

130.00

0.75

97.50

2260.00

Unit

$\begin{array}{ll}\text { Plant } & \text { Project } \\ \text { Type } & \text { Status }\end{array}$

ROR

Factor

Exist Prob

Factor

Exist Prob

Wild/Scenic Protection

wild/scenic Tributary or Upstream/Downstream wild/Scenic Location cultural value

Fish Presence Value

Geologic Value

Historic Value

other Value

Recreation Value

Scenic Value
Latitude

Longitude

4211.00

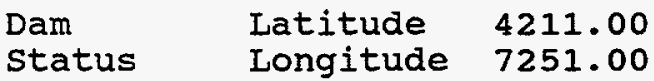

W state

Name

MA
PESF Annual

1695

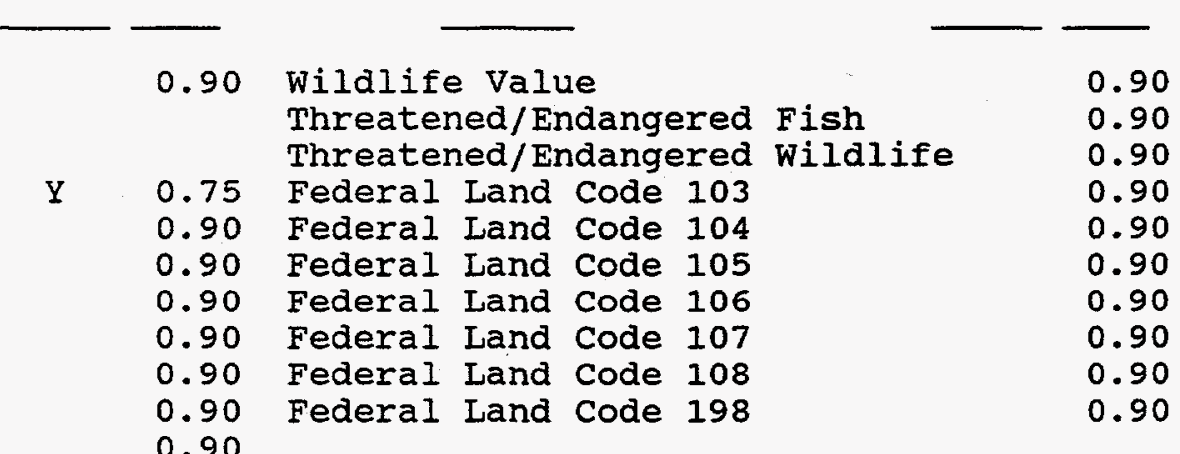


FERC

Number

Plant Name

MA021
RUSSELI (CRESCENT MILLS)

County Name

HAMPDEN
River Basin

CONNECTICUT RIVER BASIN
Class Owner Name

R TEXON INC

Name Plate Rating (KW) PESF $\mathrm{PESF} * \mathrm{KW}$

Annual Energy Rating (MWh)

1125.00

$0.75 \quad 843.75$

5940.00
Dam Latitude 4213.00

Status Longitude

7235.00
State

Name

MA

WO

Factor

wild/scenic Protection

wild/Scenic Tributary or Upstream/ Downstream wild/Scenic Location Cultural Value Fish Presence Value

Geologic Value

Historic Value

other value

Recreation Value

Scenic Value
Exist Prob

0.90

wildlife Value

Threatened/Endangered Fish

Threatened/Endangered Wildife

0.75 Federal Land Code 103

0.90 Federal Land code 104

0.90 Federal Land code 105

0.90 Federal Land Code 106

0.90 Federal Land Code 107

0.90 Federal Land code 108

0.90 Federal Land code 198
4455

PESF Annual Energy Rating (MWh)

0.90

Exist Prob

0.90

0.90

0.90

0.90

0.90

0.90

0.90

0.90

0.90

0.90 
RESOURCE DA T A B A S E L I S T I N G

DATE: $06 / 27 / 95$

PAGE NO: 109

FERC

Number Plant Name

MA022 HUNTINGTON

county Name

HAMPDEN

River Basin

CONNECTICUT RIVER BASIN

Class Owner Name

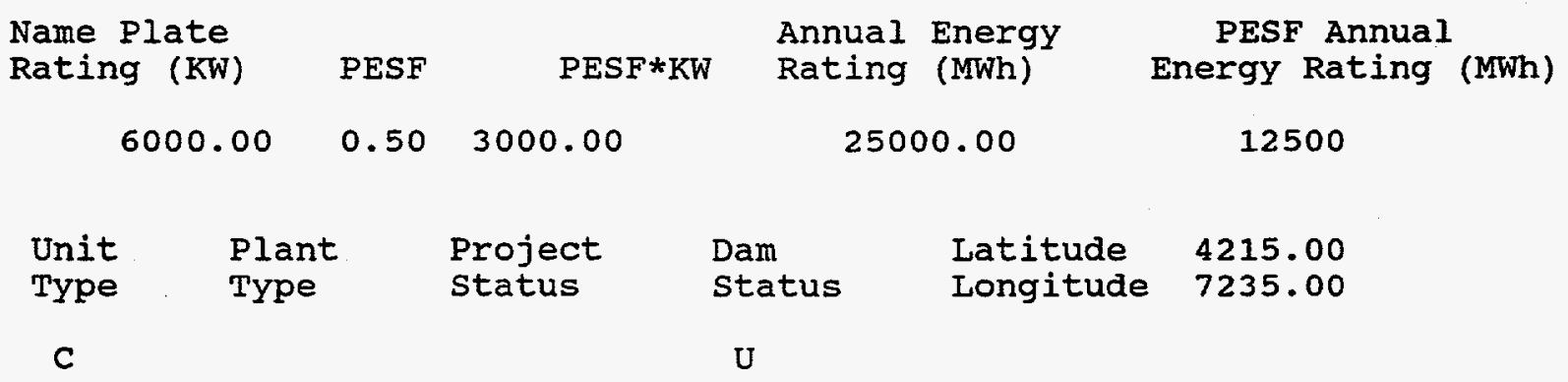

\section{Factor}

wild/scenic Protection

wild/Scenic Tributary or Upstream/Downstream wild/Scenic Location cultural Value

Fish Presence Value

Geologic Value

Historic Value

other Value

Recreation Value

Scenic Value
Exist Prob

0.90

Wildife Value

Threatened/Endangered Fish

Threatened/Endangered Wildlife

$Y$

0.50

0.90

0.90

Federal Land code 103

Federal Land Code 104

Federal Land Code 105

Federal Land Code 106

0.90 Federal Land Code 107

0.90 Federal Land Code 108

0.90 Federal Land Code 198

0.90
State

Name

MA
Exist Prob

0.90

0.90

0.90

0.90

0.90

0.90

0.90

0.90

0.90

0.90 
FERC

Number

Plant Name

MA023 county Name

HAMPDEN
Stream

CHICOPEE R

River Basin

CONNECTICUT RIVER BASIN
State

Name

MA

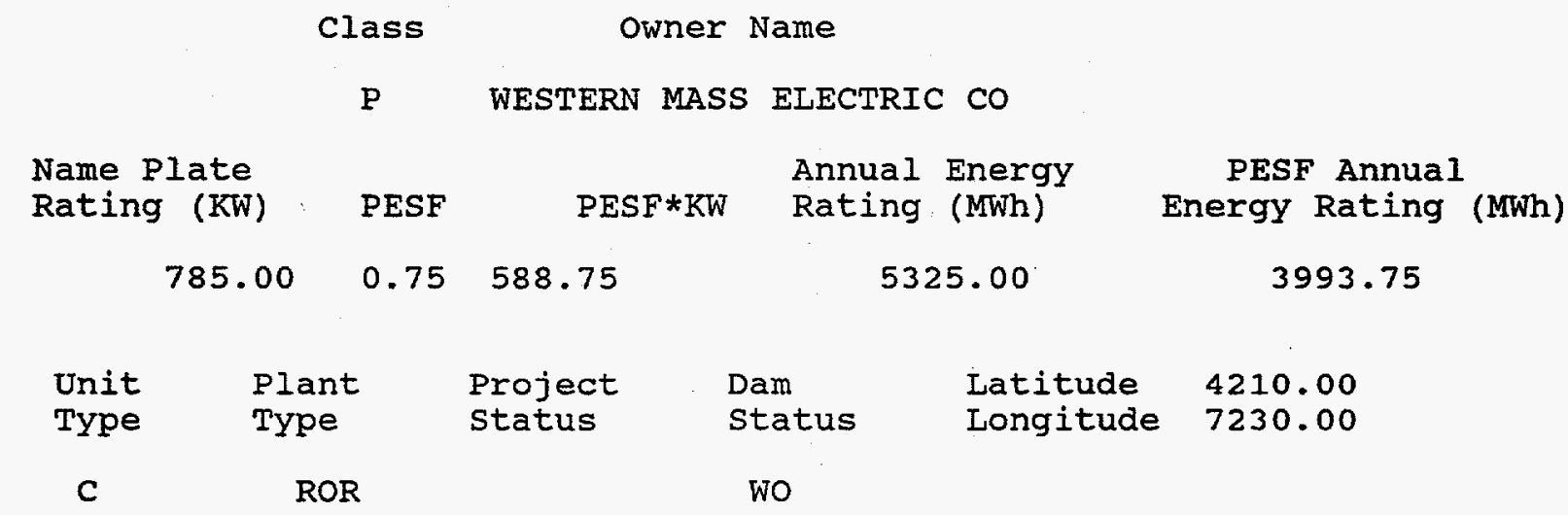

Factor

Exist Prob

wild/Scenic Protection

Wild/Scenic Tributary or Upstream/ Downstream wild/Scenic Location Cultural value

Fish Presence Value

Geologic Value

Historic Value

other Value

Recreation value

scenic Value
Factor

Exist Prob

0.90

Wildlife Value Threatened/Endangered Fish Threatened/Endangered wildlife

0.90

0.90

0.90

0.75 Federal Land Code 103

0.90 Federal Land Code 104

0.90

0.90

Federal Land code 105

0.90 Federal Land code 106

0.90 Federal Land code 107

0.90 Federal Land Code 108

0.90 Federal Land Code 198

0.90
0.90

0.90

0.90

0.90

0.90

0.90 
DATE: $06 / 27 / 95$

PAGE NO: 111

FERC

Number

Plant Name

stream

State

Name

MAO24 PUTTS BRIDGE

CHICOPEE R

MA

County Name

HAMPDEN
River Basin

CONNECTICUT RIVER BASIN

\section{Class Owner Name}

P WESTERN MASS ELECTRIC CO

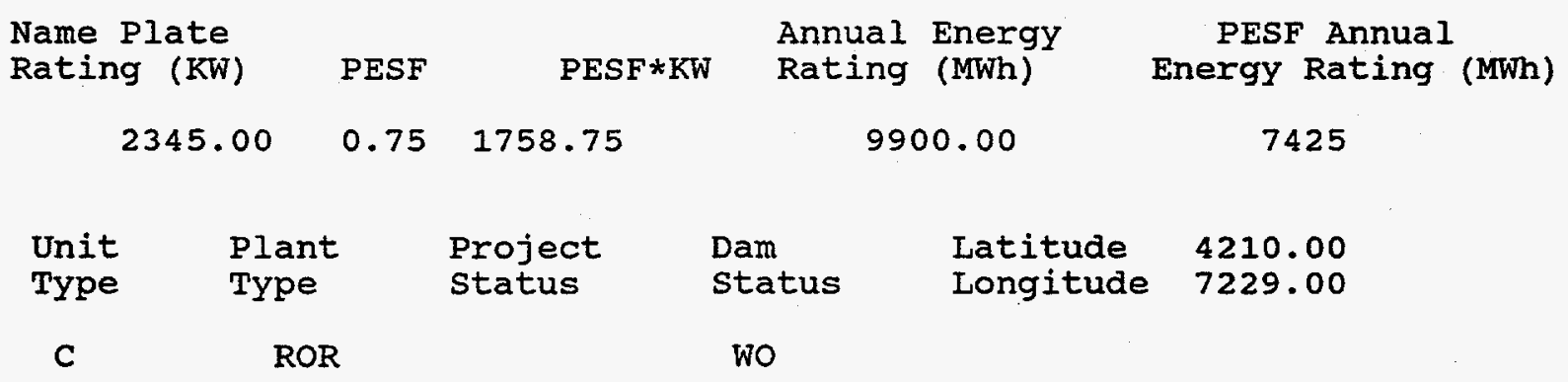

\section{Factor}

wild/Scenic protection

Wild/Scenic Tributary or Upstream/Downstream wild/Scenic Location Cultural value Fish Presence Value Geologic Value Historic Value Other Value Recreation Value Scenic value
Exist Prob

0.90

Y

0.75

0.90

0.90

0.90

0.90

0.90

0.90

0.90
Wildife value Threatened/Endangered Fish Threatened/Endangered Wildlife

Factor

Federal Iand code 103

Federal Land code 104

Federal Land code 105

Federal Land Code 106

Federal Land code 107

Federal Land Code 108

Federal Land code 198
Exist Prob

0.90

0.90

0.90

0.90

0.90

0.90

0.90

0.90

0.90

0.90 
RESOUR CE D A TA B A S E L I S I I N

DATE: $06 / 27 / 95$

PAGE NO: 112

FERC

Number

Plant Name

Stream

State

MA025 LUDLOW (RED BRIDGE)

CHICOPEE R

Name

MA

County Name

River Basin

HAMPDEN

CONNECTICUT RIVER BASIN

Class Owner Name

P WESTERN MASS ELECTRIC CO

Name Plate

$\begin{array}{ll} & \text { Annual Energy } \\ \text { PESF } & \text { PESF*KW Rating (MWh) }\end{array}$

PESF Annual

Rating ( $\mathrm{KW}$ )

$0.50 \quad 592.50$

6400.00

3200

1185.00

Plant

Project

Dam

status

Latitude

Longitude

4211.00

Type

ROR

$\mathrm{U}$

Factor

wild/scenic Protection

wild/Scenic Tributary or

Upstream/Downstream

wild/Scenic Location

Cultural Value

Fish Presence Value

Geologic Value

Historic Value

other Value

Recreation Value

Scenic Value
Exist Prob

0.90

$Y$
Factor

Exist Prob

0.90

0.90

0.90

0.50 Federal Land code 103

0.90 Federal. Land code 104

0.90 Federal Land code 105

0.90 Federal Land code 106

0.90 Federal Land code 107

0.90 Federal Land code 108

0.90 Federal Land code 198

0.90
0.90

0.90

0.90

0.90

0.90

0.90

0.90 
R E S O UR C E D A T A B A S E I I S T I N G

DATE: $06 / 27 / 95$

PAGE NO: 113

FERC

Number

$\mathrm{MAO} 26$
Plant Name

QUABBIN-WINDSOR

County Name

HAMPSHIRE stream

SWIFT R

River Basin

CONNECTICUT RIVER BASIN

\section{Class Owner Name}

M METROPOLITAN DIST COMM

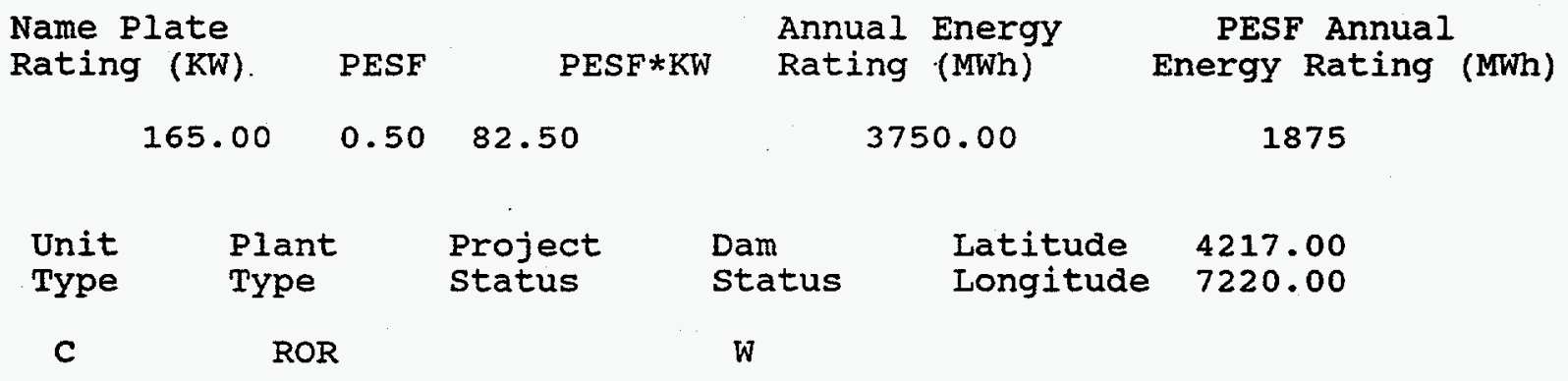

Factor

wild/Scenic Protection

wild/Scenic Tributary or Upstream/Downstream wild/Scenic Location

Cultural Value

Fish Presence Value

Geologic Value

Historic Value

other Value

Recreation Value

Scenic Value
Exist Prob

Factor

0.90 Wildlife Value

Threatened/Endangered Fish Threatened/Endangered Wildlife $Y$

$Y$

0.75

0.90

0.90

Federal Land code 103

Federal Land code 104

Federal Land code 105

0.90 Federal Land code 106

0.90 Federal Land Code 107

$\mathrm{Y} \quad 0.75$ Federal Land code 108

0.90 Federal Land Code 198
State

Name

MA 
RE S O U R C E D A TA B A S E I I S T I N G

DATE: $06 / 27 / 95$

PAGE NO: 114

FERC

Number

Plant Name

stream

state

Name

MA027 TIGHE CARMODY

MANHAN $R$

MA

County Name

HAMPSHIRE

Class

Owner Name

M HOLYOKE WATER WORKS

Name Plate

Rating ( $\mathrm{KW}$ )

230.00

PESF

PESF* KW

Annual Energy

$0.50 \quad 115.00$

Project

status

Unit
Type

Plant

Type
River Basin

CONNECTICUT RIVER BASIN

\author{
C \\ ROR \\ Factor \\ wild/Scenic Protection \\ Wild/Scenic Tributary or \\ Upstream/Downstream \\ wild/Scenic Location \\ cultural Value \\ Fish Presence Value \\ Geologic Value \\ Historic Value \\ other Value \\ Recreation Value \\ Scenic Value
}

Exist Prob

0.90
Factor

Latitude

Longitude

4213.00

Status

U
PESF Annual Energy Rating (MWh)

537.50 
R E S U U C E D A T A B A S E I I S T I N G

DATE: $06 / 27 / 95$

PAGE NO: 115

FERC

Number

MAO28
Plant Name

ROARING BROOK DAM

County Name

HAMPSHIRE stream

ROARING BK, MILL R

River Basin

CONNECTICUT RIVER BASIN
State

Name

MA

\section{Class Owner Name}

M S DEERFIELD WTR SUPPLY DIST

\begin{tabular}{|c|c|c|c|c|c|c|}
\hline \multicolumn{2}{|c|}{ Name Plate } & PESF & $\mathrm{PESF} * \mathrm{KW}$ & $\begin{array}{l}\text { Annu } \\
\text { Rati }\end{array}$ & $\begin{array}{l}1 \text { Energy } \\
\text { ig (MWh) }\end{array}$ & $\begin{array}{l}\text { PESF Annual } \\
\text { Energy Rating (MWh) }\end{array}$ \\
\hline & 52.00 & 0.90 & 46.80 & & 150.00 & 135 \\
\hline $\begin{array}{l}\text { Unit } \\
\text { Type }\end{array}$ & $\begin{array}{l}\text { Plant } \\
\text { Type }\end{array}$ & & $\begin{array}{l}\text { Project } \\
\text { status }\end{array}$ & $\begin{array}{l}\text { Dam } \\
\text { status }\end{array}$ & $\begin{array}{l}\text { Latitude } \\
\text { Longitude }\end{array}$ & $\begin{array}{l}4227.00 \\
7242.00\end{array}$ \\
\hline C & & & & Wo & & \\
\hline
\end{tabular}

Factor

wild/Scenic Protection

wild/Scenic Tributary or Upstream/Downstream wild/Scenic Location Cultural value

Fish Presence Value

Geologic Value

Historic Value

other Value

Recreation Value

Scenic Value
Exist Prob

0.90

Wildlife Value

Threatened/Endangered Fish Threatened/Endangered Wildlife

0.90 Federal Land Code 103

0.90 Federal Land Code 104

0.90 Federal Land Code 105

0.90 Federal Land Code 106

0.90 Federal Iand code 107

0.90 Federal Land code 108

0.90 Federal Land Code 198

0.90

Exist Prob

0.90

0.90

0.90

0.90

0.90

0.90

0.90

0.90

0.90

0.90 
FERC

Number

Plant Name

MA029 MARTIN MACH CO DAM

county Name

FRANKIIN
Stream

SAWMILL $R$

River Basin

CONNECTICUT RIVER BASIN
State

Name

MA

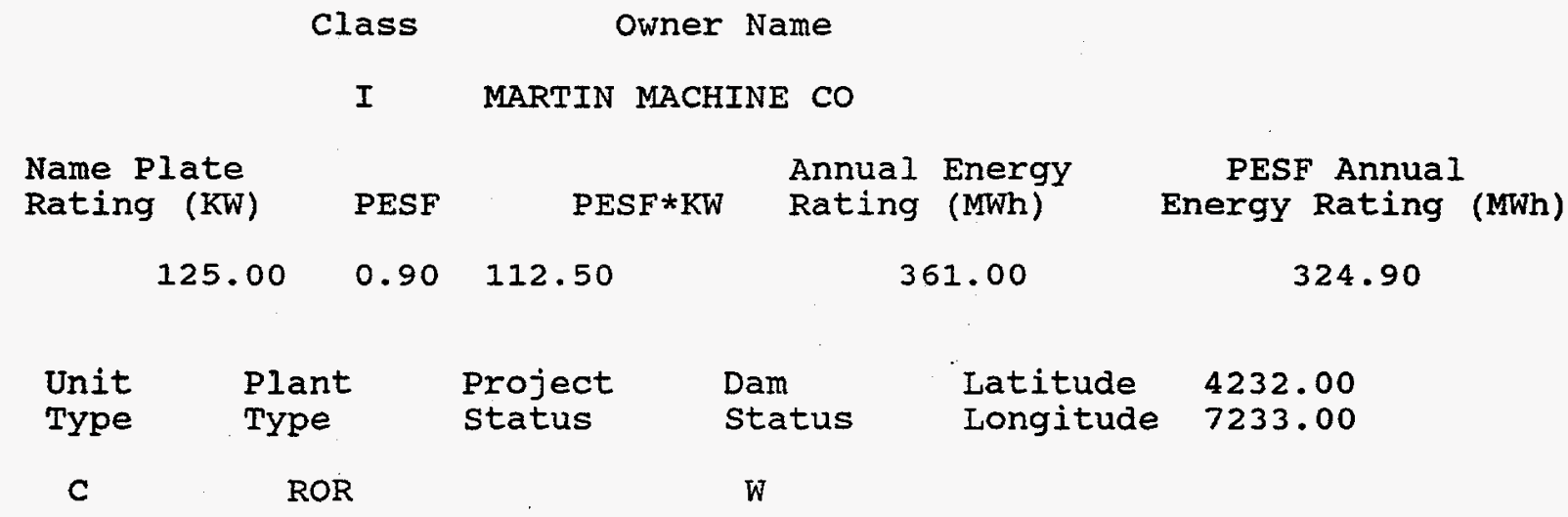

Factor

Exist Prob

Factor

Exist Prob

0.90

Wildife Value

Threatened/Endangered Fish

Threatened/Endangered Wildlife

0.90

wild/Scenic Tributary or Upstream/Downstream wild/Scenic Location

0.90 Federal Land code 103

0.90 Federal Land Code 104

0.90 Federal Land Code 105

0.90 Federal Land Code 106

0.90 Federal Land code 107

0.90 Federal Land code 108

0.90 Federal Land Code 198

0.90

0.90

0.90

0.90

0.90

0.90

0.90

0.90

0.90

0.90

Scenic Value 
RESOURCE D A T A B A S E I I S T I N G

DATE: $06 / 27 / 95$

PAGE NO: 117

\section{FERC}

Number

Plant Name

MA030 DENISON DAM

county Name

FRANKLIN

\section{class}

I

C A DENISON INC
GREEN R,DEERFIELD R

River Basin

CONNECTICUT RIVER BASIN state

Name

MA
PESF Annual Energy Rating (MWh)

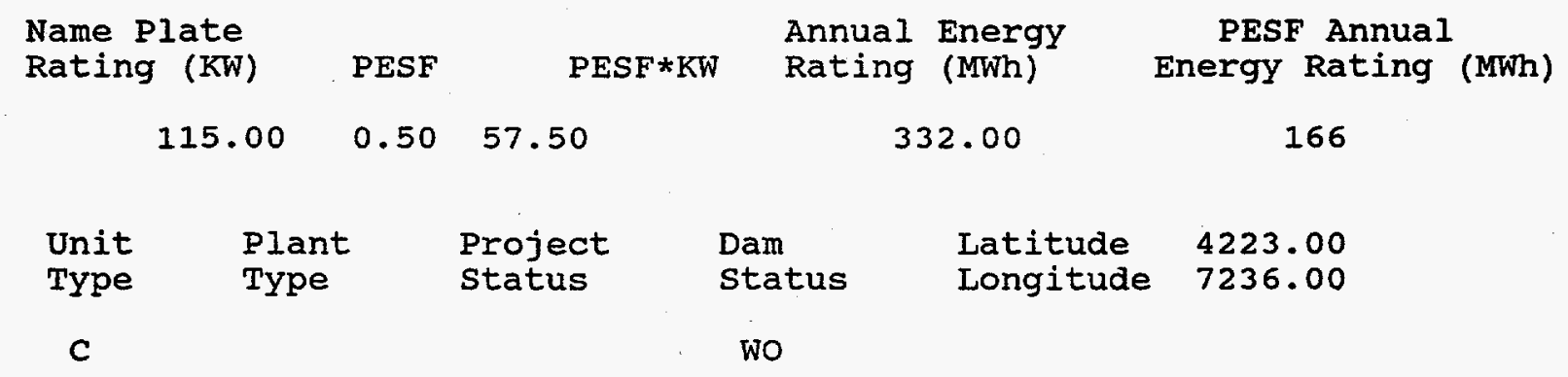

\section{Factor}

wild/scenic Protection wild/Scenic Tributary or Upstream/Downstream wild/Scenic Location Cultural value Fish Presence Value Geologic Value Historic Value other Value Recreation Value Scenic Value
Exist Prob

0.90

\section{Wildlife Value} Threatened/Endangered Fish Threatened/Endangered Wildlife $Y$

Y

0.75

0.90$$
\text { Federal Land code } 103
$$$$
\text { Federal Land Code } 104
$$

Y 0.75 Federal Land code 105

0.90 Federal Land Code 106

Y 0.75 Federal Land Code 107

$\mathrm{Y} \quad 0.75$ Federal Land Code 108

0.90 Federal Land Code 198
Exist Prob

$$
\begin{aligned}
& 0.90 \\
& 0.75 \\
& 0.75 \\
& 0.90 \\
& 0.90 \\
& 0.90 \\
& 0.90 \\
& 0.90 \\
& 0.90 \\
& 0.90
\end{aligned}
$$


FERC

Number

Plant Name

MA031

GARDNER FALLS

County Name

FRANKLIN

Class

Owner Name

P

NEW ENGLAND POWER CO

\begin{abstract}
Name Plate
\end{abstract}
Rating ( $\mathrm{KW}$ )

1720.00

PESF

PESF*KW

Annual Energy

Rating (MWh)

0.25430 .00

4300.00

Dam

Status

Latitude

Longitude

\begin{tabular}{|c|c|c|c|c|}
\hline $\begin{array}{l}\text { Unit } \\
\text { Type }\end{array}$ & $\begin{array}{l}\text { Plant } \\
\text { Type }\end{array}$ & $\begin{array}{l}\text { Project } \\
\text { status }\end{array}$ & $\begin{array}{l}\text { Dam } \\
\text { status }\end{array}$ & $\begin{array}{l}\text { Latitude } \\
\text { Longitude }\end{array}$ \\
\hline
\end{tabular}

\section{CONNECTICUT RIVER BASIN}

PESF Annual Energy Rating (MWh)

1075
C

ROR

$\mathrm{U}$

Factor

wild/Scenic Protection wild/Scenic Tributary or Upstream/Downstream wild/Scenic Location Cultural Value Fish Presence Value

Geologic Value Historic Value Other Value Recreation Value Scenic Value
Exist Prob

$$
0.90
$$

Wildlife Value Threatened/Endangered Fish Threatened/Endangered Wildiife

Y $\quad \begin{array}{lll}0.50 & \text { Federal Land Code } 103 \\ 0.90 & \text { Federal Land Code } 104\end{array}$

Y 0.25 Federal Land code 105

0.90 Federal Land Code 106

Y 0.50 Federal Land code 107

Y $\quad 0.50$ Federal Land Code 108

0.90 Federal Land Code 198
State

Name

MA 
R E S O U R E D A T A B A S E I I S T I N G

DATE: $06 / 27 / 95$

PAGE NO: 119

FERC

Number

$\mathrm{MAO} 32$
Plant Name

DEERFIELD 3

county Name

FRANKLIN

\author{
State \\ Name \\ MA \\ DEERFIELD $R$ \\ River Basin
}

CONNECTICUT RIVER BASIN

Class Owner Name

P WESTERN MASS ELECTRIC CO

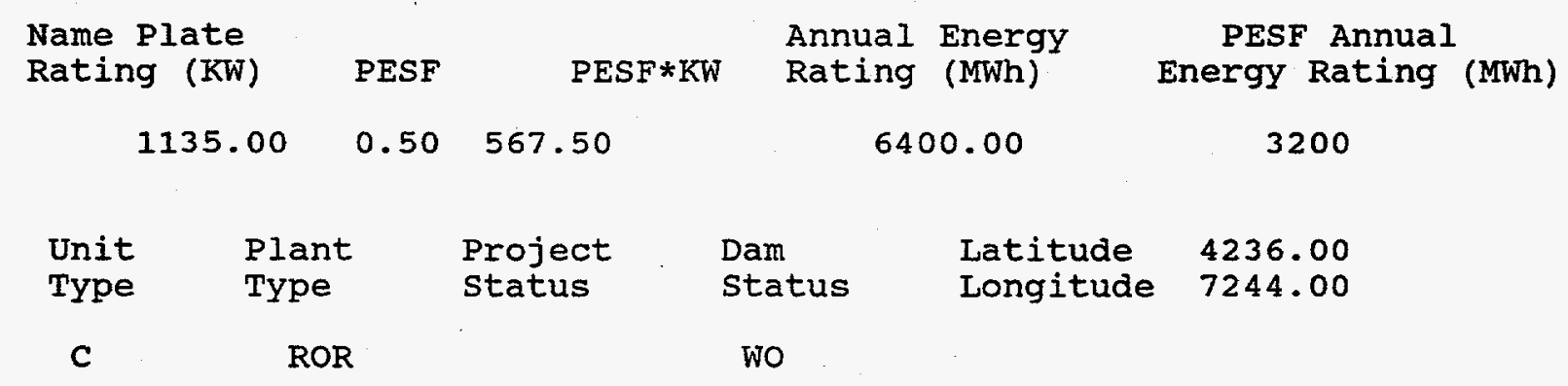

Factor

wild/Scenic Protection

wild/scenic Tributary or Upstream/Downstream wild/Scenic Location Cultural Value Fish Presence Value Geologic Value Historic Value other value Recreation Value Scenic Value

\section{Exist Prob}

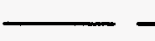

$$
0.90
$$

\section{$Y$}

0.75

0.90

$\mathrm{Y} \quad 0.75$

0.75
0.90

Y $\quad 0.75$

$\mathrm{Y} \quad 0.75$

0.90

0.90
Factor

Exist Prob

0.90

0.90

0.90

0.90 Threatened/Endangered Wildlife

Federal Land Code 103

Federal Land Code 104

Federal Land Code 105

Federal Land Code 106

Federal Land Code 107

Federal Land Code 108

Federal Land Code 198
0.90

0.90

0.90

0.90

0.90

0.90 
R E S O U R C E

DATE: $06 / 27 / 95$
$D A T A B A S E$

L I S T N G

PAGE NO: 120 plant Name

Number

MA033
CHARIEMONT
County Name

FRANKLIN

Owner Name

State Name

MA
River Basin

CONNECTICUT RIVER BASIN

Class
r

Factor

Exist Prob

Factor

Exist Prob

wild/Scenic Protection

wild/Scenic Tributary or Upstream/Downstream wild/Scenic Location Cultural value Fish Presence Value Geologic Value Historic Value other Value Recreation Value scenic value

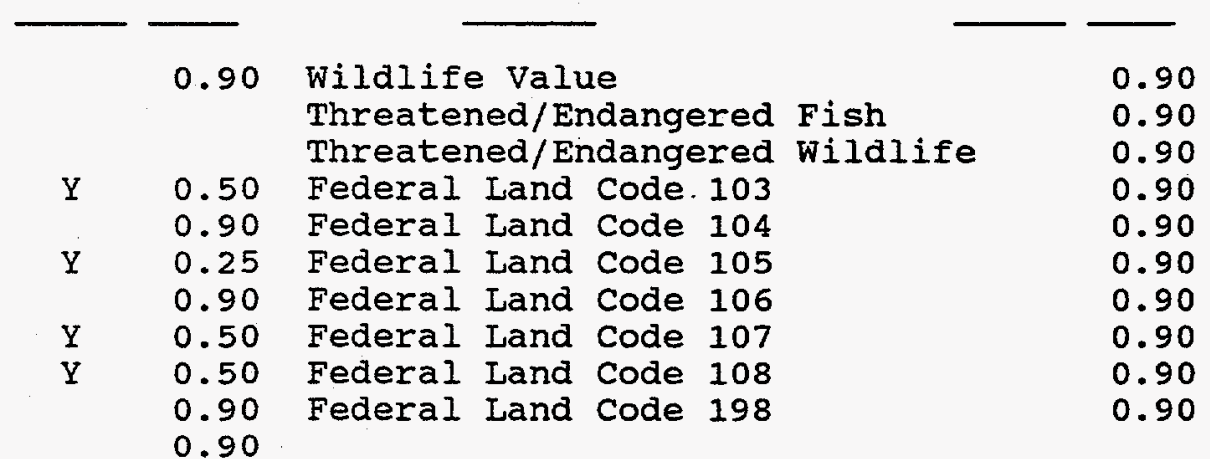


RES O U R C E D A T A B A S E I I S T I N G

DATE: $06 / 27 / 95$

PAGE NO: 121

FERC

Number

Plant Name

MA034 MILL BROOK

county Name

FRANKLIN

$\begin{array}{lc}\text { Stream } & \begin{array}{c}\text { State } \\ \text { Name }\end{array} \\ \text { MILL BK, DEERFIELD R } & \text { MA } \\ \text { River Basin } & \end{array}$

CONNECTICUT RIVER BASIN

\section{Class Owner Name}

M CHARLEMONT FIRE DISTRICT

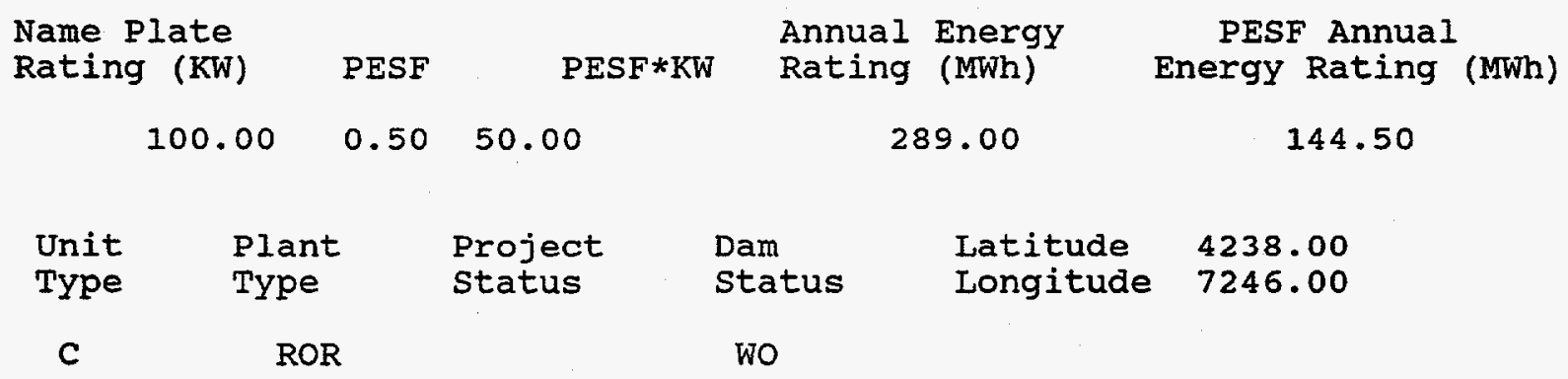

\section{Factor}

Wild/Scenic Protection

wild/Scenic Tributary or Upstream/Downstream wild/Scenic Location Cultural value Fish Presence Value Geologic Value Historic Value other Value Recreation Value Scenic Value
Exist Prob

0.90

Wildife Value Threatened/Endangered Fish Threatened/Endangered Wildife

Exist Prob

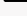

$\mathrm{Y}$

0.75

Federal Land code 103

0.90 Federal Land code 104

$\mathrm{Y}$

0.75

Federal Land Code 105

0.90 Federal Land code 106

$\mathrm{Y} \quad 0.75$

$Y \quad 0.75$

0.90

0.90

Federal Land code 107

Federal Land Code 108

Federal Land Code 198

0.90

0.90

0.90

0.90

0.90

0.90 
R E S O U R C E

DATE: $06 / 27 / 95$
D A T A B A S E

L I S T I N G

PAGE NO: 122

FERC

Number

plant Name

MA035 ZOAR

county Name

FRANKLIN

Class
Stream

DEERFIELD $R$

River Basin

CONNECTICUT RIVER BASIN state

Name

MA

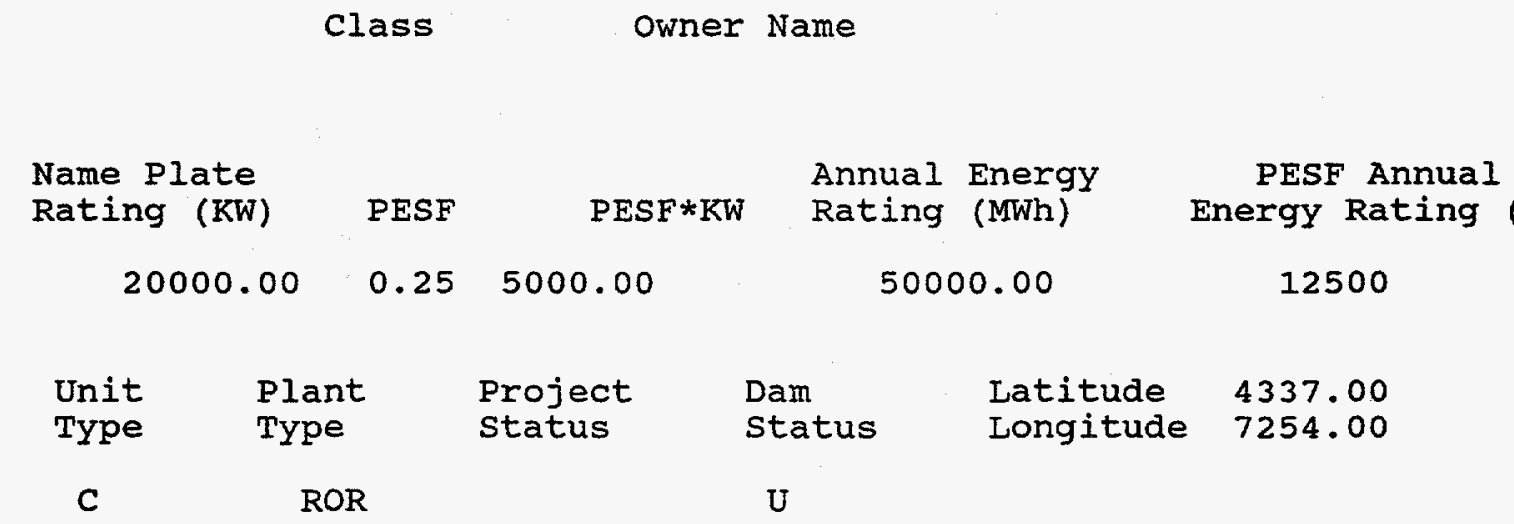

Factor

Exist Prob

wild/scenic Protection

wild/Scenic Tributary or Upstream/Downstream wild/Scenic Location Cultural value

Fish Presence Value

Geologic Value

Historic Value

other Value

Recreation Value

Scenic Value
Factor

Exist Prob

0.90

Wildlife Value

Threatened/Endangered Fish

Threatened/Endangered Wildlife

0.90

0.90

0.90

Y 0.50 Federal Land code 103

0.90 Federal Land Code 104

0.90

0.90

Federal Land code 105

0.90 Federal Land code 106

0.90

0.90

$\mathrm{Y} \quad 0.50$

Federal Land Code 107

0.90

0.50

Federal Land code 108

0.90

0.90

Federal Land code 198

0.90 
FERC

Number
Plant Name

MA036 county Name

FRANKLIN
River Basin

CONNECTICUT RIVER BASIN

$$
\begin{array}{cc}
\text { Class } & \text { Owner Name } \\
\text { P } & \text { NEW ENGLAND POWER CO }
\end{array}
$$

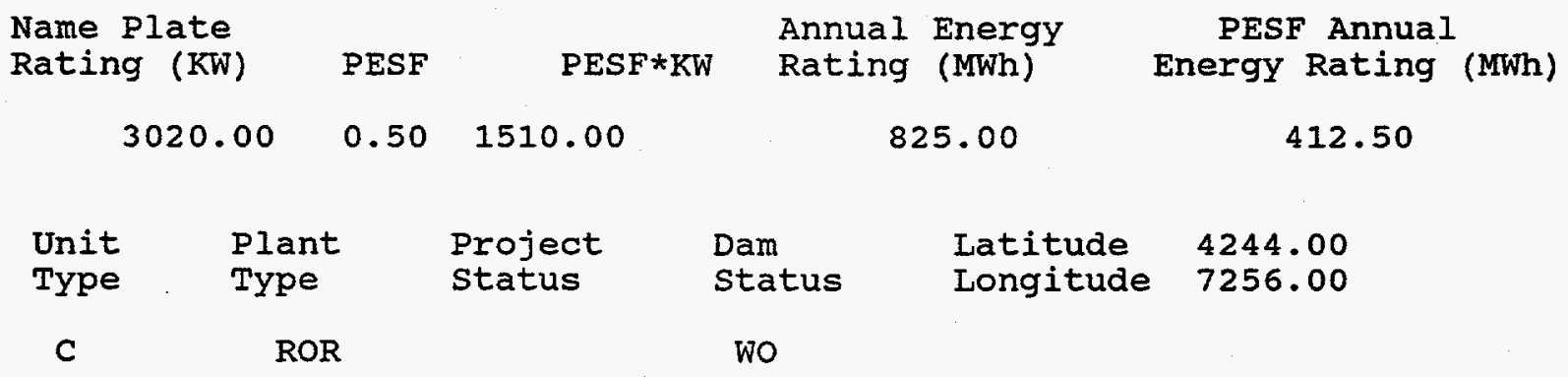

Factor

wild/Scenic Protection

wild/Scenic Tributary or Upstream/ Downstream wild/Scenic Location Cultural value Fish Presence Value Geologic Value Historic Value other Value Recreation Value Scenic Value
Exist Prob

Factor

Exist Prob

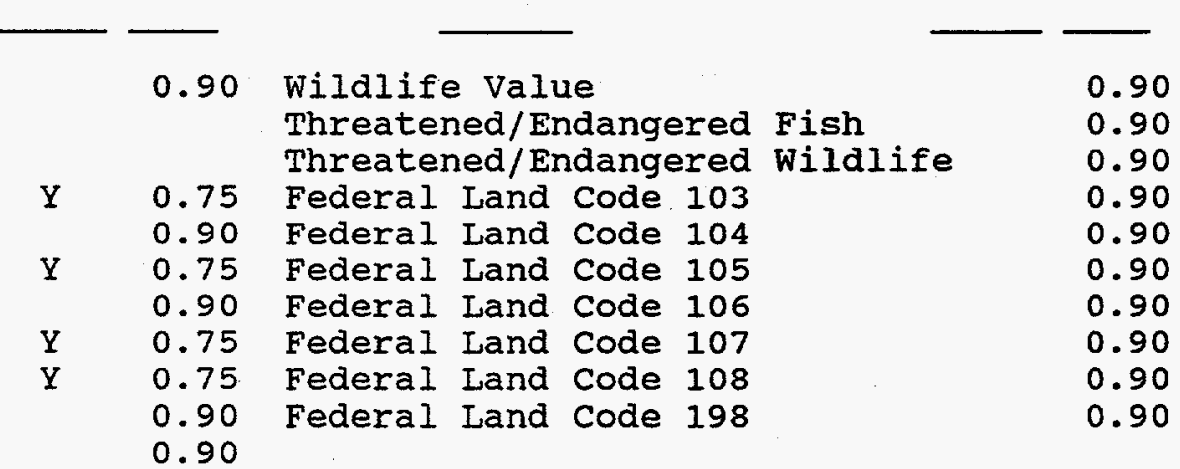

State Name

MA 
R E S O U R C E

DATE: $06 / 27 / 95$

FERC

Number

MA037

TURNERS FALLS

county Name

FRANKLIN

class

Owner Name
PAGE NO: 124

stream

state

Name

CONNECTICUT $R$

MA

CONNECTICUT RIVER BASIN

Class
r

\section{Factor}

Wild/Scenic Protection

Wild/Scenic Tributary or

Upstream/Downstream

wild/Scenic Location

Cultural Value

Fish Presence Value

Geologic Value

Historic Value

other Value

Recreation Value

Scenic value
Exist Prob

Factor

Exist Prob

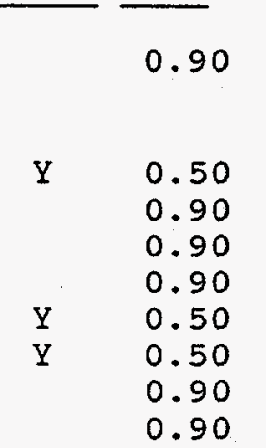

Wildife Value Threatened/Endangered Fish Threatened/Endangered Wildife Y Federal Land code 103 Federal Land code 104 Federal Land Code 105 Federal Land code 106 Federal Land Code 107 Federal Land Code 108 Federal Land code 198
0.90

0.50

0.50

0.90

0.90

0.90

0.90

0.90

0.90

0.90 
RESOURCE DATABASE LIS T ING

DATE: $06 / 27 / 95$

PAGE NO: 125

FERC

Number

Plant Name

Stream

State

Name

MA038 BOOK BINDERY POND

MILLERS R

MA

County Name

FRANKIIN
River Basin

CONNECTICUT RIVER BASIN

\section{Class Owner Name}

I DIAMOND NATIONAL CORP

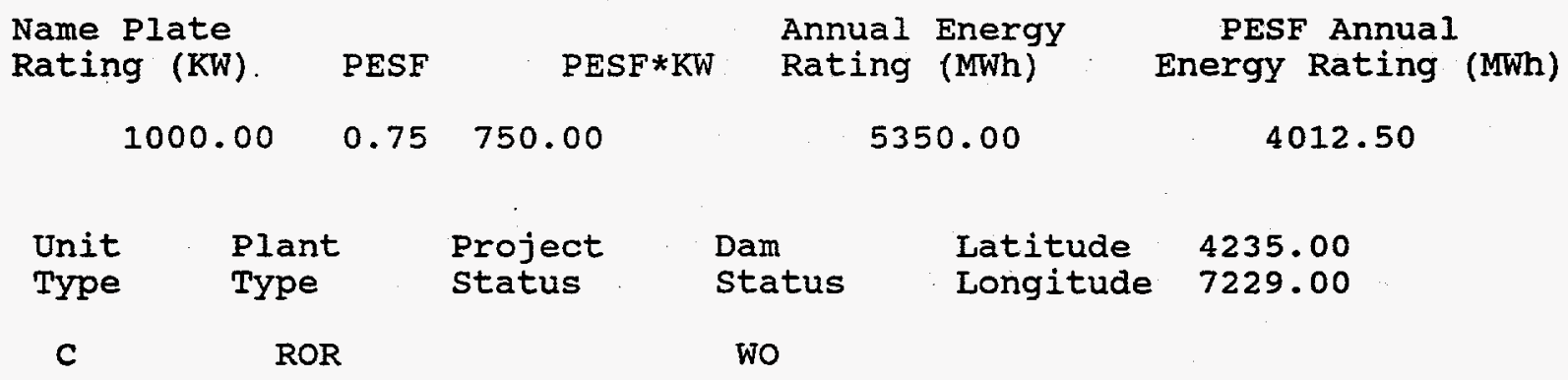

Factor

Exist Prob

Wild/Scenic Protection

wild/Scenic Tributary or Upstream/ Downstream wila/Scenic Location cultural value Fish Presence Value Geologic Value Historic Value other value Recreation Value Scenic Value
Factor

0.90

Wildife Value

0.90

0.90
Exist Prob

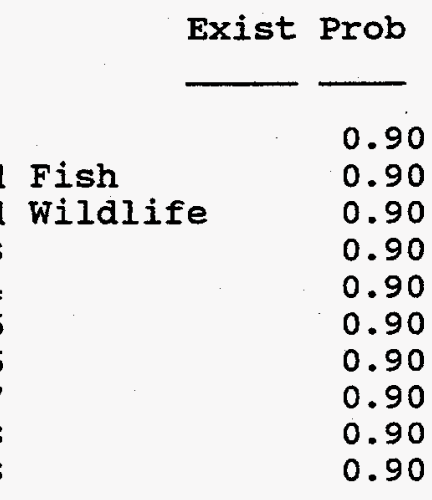

0.90

0.90

0.90

0.90

0.90

0.90 Threatened/Endangered Fish Threatened/Endangered Wildlife

0.90 Federal Land Code 103

0.75 Federal Land Code 104

0.90

Federal Land code 105

Federal Land Code 106

0.90

0.90

Federal Land code 107

Federal Land code 108

Federal Land Code 198

0.90 


\section{FERC}

Number

Plant Name

MA039 MILLERS FALLS ONE

County Name

FRANKLIN stream

MILLERS R

River Basin

CONNECTICUT RIVER BASIN
State

Name

MA

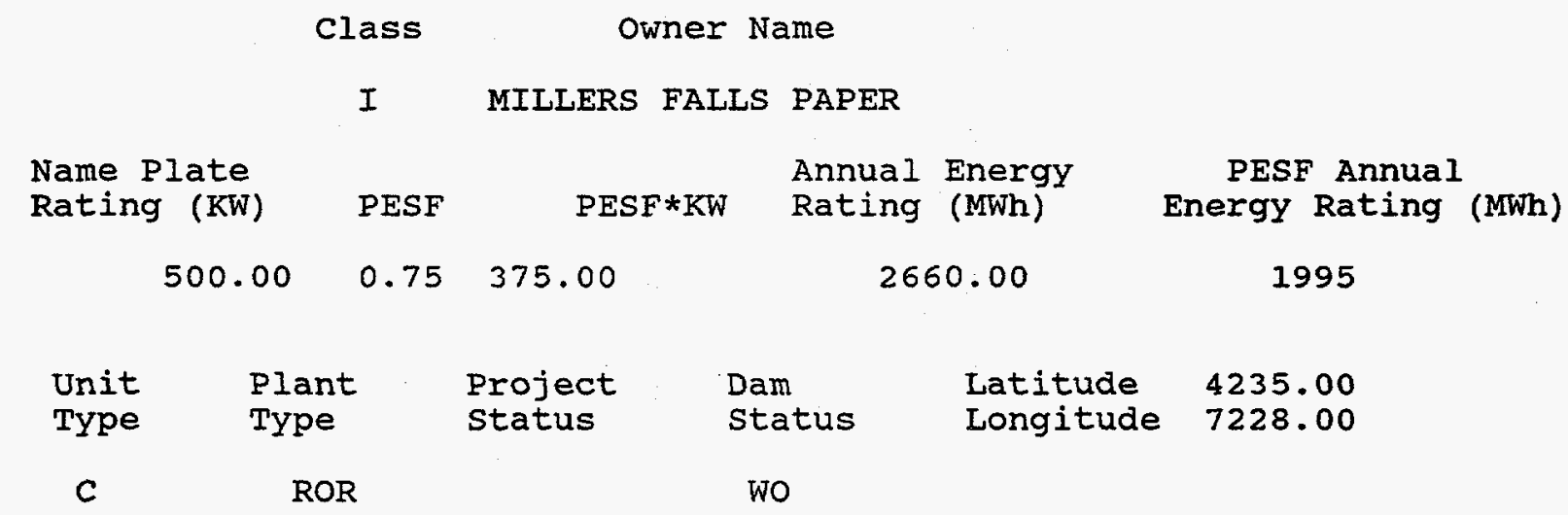

Factor

Exist Prob

Factor

Exist Prob

wild/Scenic Protection

Wild/Scenic Tributary or Upstream/Downstream Wild/Scenic Location Cultural Value

Fish Presence Value

Geologic Value

Historic Value

Other Value

Recreation Value

Scenic Value
0.90 Wildife Value

$\longrightarrow$

Threatened/Endangered Fish

Threatened/Endangered Wildlife

0.90 Federal Land Code 103

Y $\quad 0.75$ Federal Land Code 104

0.90 Federal Land Code 105

0.90 Federal Land Code 106

0.90 Federal Land Code 107

0.90 Federal Land Code 108

0.90 Federal Land Code 198
0.90

0.90

0.90

0.90

0.90

0.90

0.90

0.90

0.90

0.90 
R E S O U R E D A T A B A S E I I S T I N G

DATE: $06 / 27 / 95$

PAGE NO: 127

FERC

Number

Plant Name

Stream

MIILERS R

MA040 ERVING

county Name

FRANKLIN
River Basin

CONNECTICUT RIVER BASIN

Class Owner Name

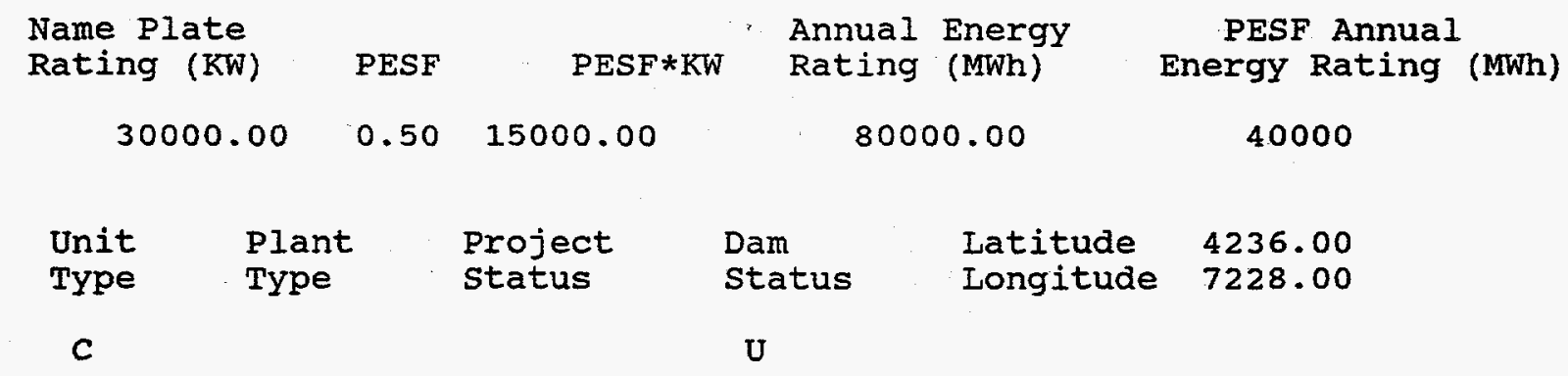

Factor

wild/Scenic Protection

Wild/Scenic Tributary or Upstream/Downstream wild/Scenic Location Cultural value

Fish Presence Value

Geologic Value

Historic Value

other value

Recreation Value

Scenic Value

\begin{abstract}
Exist Prob
\end{abstract}
Factor

Exist Prob

$\begin{array}{lr}\text { Factor } & \text { Exist Prob } \\ \text { Wildlife Value } & -9.90 \\ \text { Threatened/Endangered Fish } & 0.90 \\ \text { Threatened/Endangered Wildlife } & 0.90 \\ \text { Federal Land code 103 } & 0.90 \\ \text { Federal Land Code 104 } & 0.90 \\ \text { Federal Land Code 105 } & 0.90 \\ \text { Federal Land Code 106 } & 0.90 \\ \text { Federal Land Code 107 } & 0.90 \\ \text { Federal Land Code 108 } & 0.90 \\ \text { Federal Land Code 198 } & 0.90\end{array}$

State Name

MA 
DATE: $06 / 27 / 95$

PAGE NO: 128

FERC

Number

Plant Name

MA041 LAKE ROHUNTA

county Name

WORCESTER

Class

Owner Name

$\mathbf{P}$

RODNEY HUNT POWER CO

Name Plate Rating (KW) PESF PESF*KW

Annual Energy 260.00 $0.25 \quad 65.00$

Plant Type

Project status

Factor

Latitude

Longitude

4235.00

status

U
State Name

MA
PESF Annual Energy Rating (MWh)
ROR

Factor

C

Factor

Wild/Scenic Protection

wild/scenic Tributary or Upstream/Downstream wild/Scenic Location Cultural value

Fish Presence Value

Geologic Value

Historic Value

other Value

Recreation Value

Scenic Value
Exist Prob

$\begin{array}{ll} & 0.90 \\ & \\ & \\ & 0.90 \\ & 0.50 \\ & 0.90 \\ & 0.90 \\ & 0.50 \\ & 0.90 \\ & 0.90 \\ & 0.90\end{array}$

\section{Wildife Value} Threatened/Endangered Fish Threatened/Endangered Wildlife Federal Land code 103 Federal Land Code 104 Federal Land Code 105 Federal Land code 106 Federal Land Code 107 Federal Land code 108 Federal Land code 198
Exist Prob

0.90

0.90

0.90

0.90

0.90

0.90

0.90

0.90

0.90

0.90 


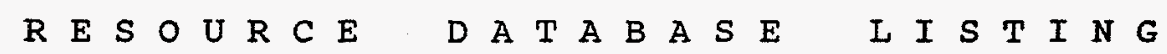

DATE: $06 / 27 / 95$

PAGE NO: 129

FERC

Number

Plant Name

Stream

state

Name

MA042 TULLY

MILLERS R

MA

county Name

River Basin

WORCESTER

CONNECTICUT RIVER BASIN

Class Owner Name

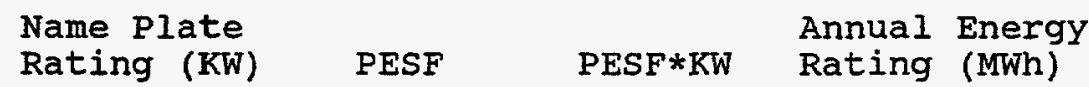

$20000.00 \quad 0.25 \quad 5000.00$

Unit Plant

Type

Type

C

Factor

Exist Prob

Wild/Scenic Protection

wild/scenic Tributary or Upstream/ Downstream wild/Scenic Location Cultural Value

Fish Presence Value

Geologic Value

Historic Value

other Value

Recreation Value

Scenic Value

\begin{abstract}
Project status
\end{abstract}

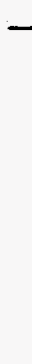

$$
30000.00
$$

\begin{tabular}{|c|c|c|c|c|c|}
\hline & 0.90 & $\begin{array}{l}\text { Wildlife } \\
\text { Threater } \\
\text { Threater }\end{array}$ & $\begin{array}{l}\text { V } \mathrm{Vall} \\
\mathrm{ned} / \mathrm{Er} \\
\mathrm{ned} / \mathrm{Er}\end{array}$ & $\begin{array}{l}\text { dangered } \\
\text { dangered }\end{array}$ & $\begin{array}{l}\text { Fish } \\
\text { wildlif }\end{array}$ \\
\hline & 0.90 & Federal & Land & Code 103 & \\
\hline$Y$ & 0.50 & Federal & Land & code 104 & \\
\hline & 0.90 & Federal. & Land & code 105 & \\
\hline & 0.90 & Federal & Land & Code 106 & \\
\hline$Y$ & 0.50 & Federal & Land & code 107 & \\
\hline & 0.90 & Federal & Land & code 108 & \\
\hline & $\begin{array}{l}0.90 \\
0.90\end{array}$ & Federal & Land & Code 198 & \\
\hline
\end{tabular}

U

PESF Annual Energy Rating (MWh)$$
7500
$$

$\begin{array}{lll}\text { Dam } & \text { Iatitude } & 4236.00 \\ \text { Status } & \text { Longitude } & 7213.00\end{array}$

Exist Prob

Factor

\begin{tabular}{|c|}
\hline $\begin{array}{l}0.90 \\
0.90 \\
0.90 \\
0.90 \\
0.90 \\
0.90 \\
0.90 \\
0.90 \\
0.90 \\
0.90\end{array}$ \\
\hline
\end{tabular}


R E S O U R C E

DATE: $06 / 27 / 95$

\section{FERC}

Number

Plant Name

MAO43

VALLEY MILL

county Name

BERKSHIRE

class

Owner Name

I KIMBERLY CLARK CORP

Name Plate Rating (KW) 375.00 PESF PESF*KW

Annual Energy Rating (MWh)

2200.00

$0.10 \quad 37.50$

Project status

ROR

Factor

Exist Prob

Factor

Unit
Type

Plant

c

Exist Prob

wild/Scenic Protection

wild/Scenic Tributary or Upstream/Downstream wild/Scenic Location Cultural Value Fish Presence Value Geologic Value Historic Value other Value Recreation Value Scenic Value $\begin{array}{lll}\text { Dam } & \text { Latitude } & 4220.00 \\ \text { Status } & \text { Longitude } & 7315.00\end{array}$

$\begin{array}{lll}\text { Dam } & \text { Latitude } & 4220.00 \\ \text { Status } & \text { Longitude } & 7315.00\end{array}$

$\begin{array}{lll}\text { Dam } & \text { Latitude } & 4220.00 \\ \text { Status } & \text { Longitude } & 7315.00\end{array}$

WO

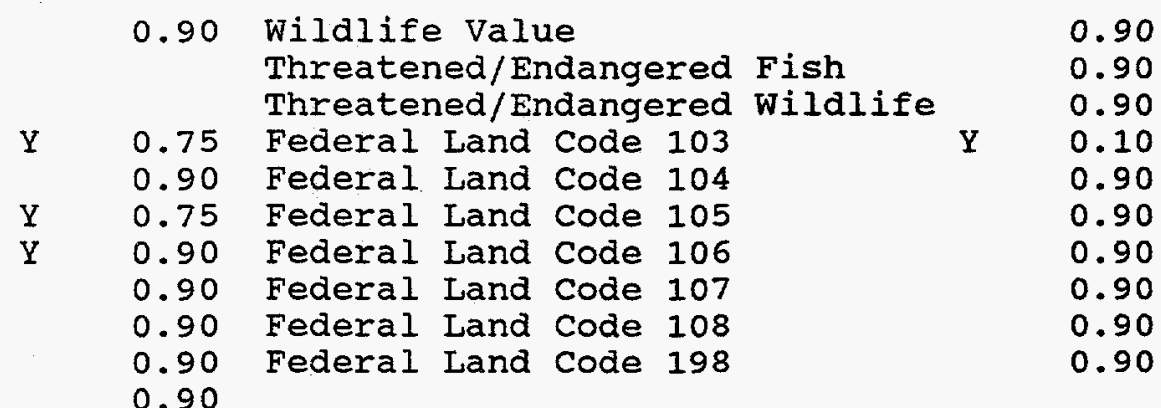

PAGE NO: 130

state Name

MA

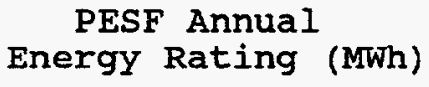

220 